
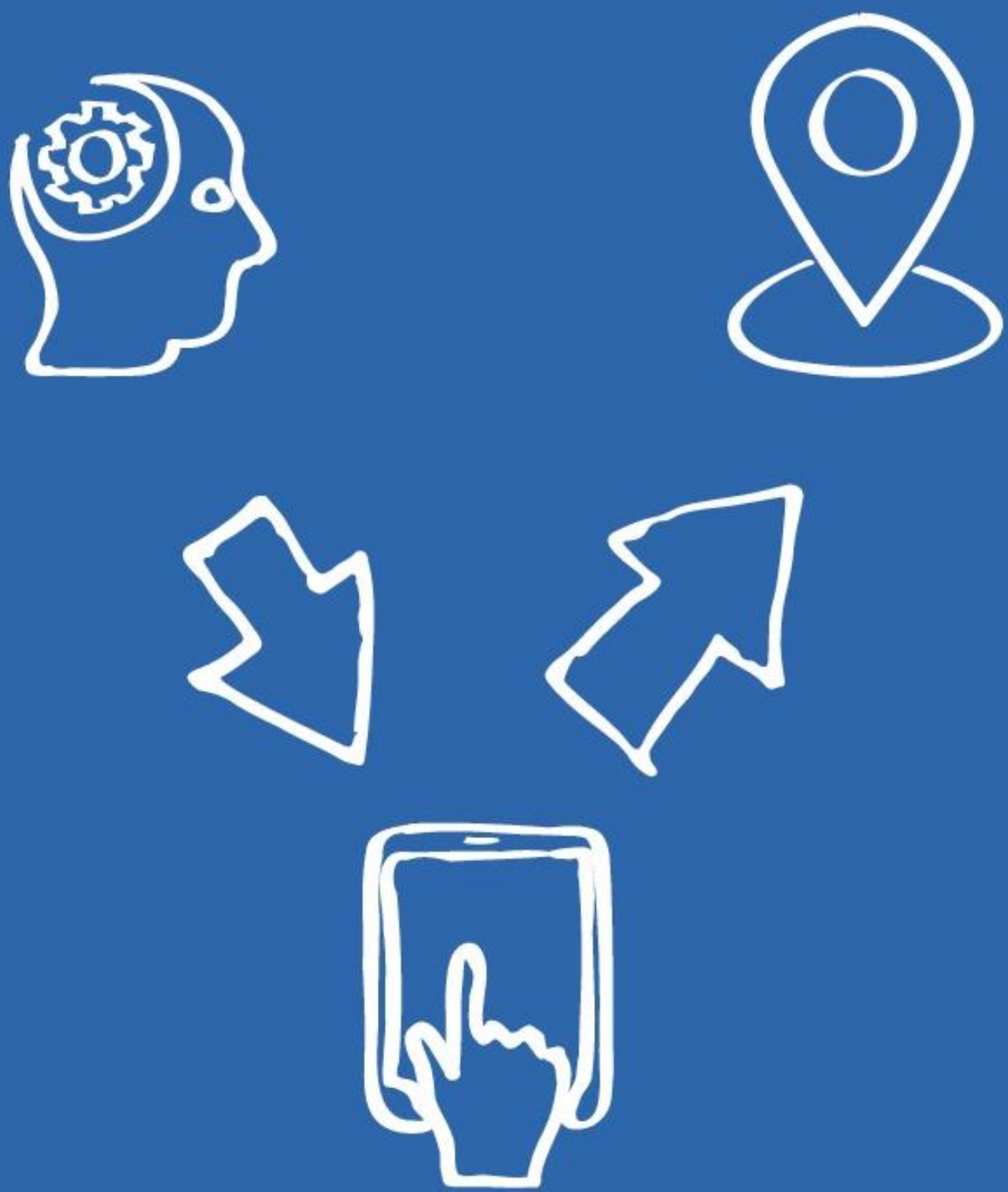


\section{Guest Editors:}

Prof Jason Levy, University of Hawaii, USA

jlevy@hawaii.edu

Prof D M Akbar Hussain, Aalborg University, Denmark

akh@et.aau.dk

\section{C Tecnología. Glosas de Innovación aplicadas a la pyme.}

Quarterly periodicity.

Special Issue on "Recent Trends in Computer Science and Electronics”, January 2019.

National and internacional circulation.

Articles reviewed by the double blind peer evaluation method.

ISSN: $2254-4143$

Depósito Legal: A 268 - 2012

DOI: http://dx.doi.org/10.17993/3ctecno.2019.specialissue

Publisher: Área de Innovación y Desarrollo, S.L. C/ Els Alzamora 17, Alcoy, Alicante (España) Tel: 965030572 info@3ciencias.com _ www.3ciencias.com. This publication may be reproduced by mentioning the source and the authors. Copyright (C) Área de Innovación y Desarrollo, S.L.

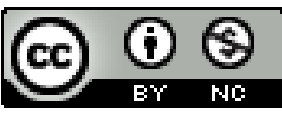




\section{PUBLISHING GOAL}

3Ciencias wants to transmit to society innovative projects and ideas. This goal is reached thought the publication of original articles which are subdue to peer review or thorough the publication of scientific books.

\section{OUR PUBLIC}

- Research staff.

- PhD students.

- Professors.

- Research Results Transfer Office.

- Companies that develop research and want to publish some of their works.

\section{TEMATIC COVERAGE}

3C Tecnología is a scientific-social journal in which original works that cover Architecture and the different fields of Engineering are disseminated, such as Mechanical, Industrial, Computer, Electrical, Agronomic, Naval, Physics, Chemistry, Civil, Electronics, Forestry, Aeronautics and Telecommunications.

\section{INSTRUCTIONS FOR AUTHORS}

All information about sending originals can be found at the following link: https://www.3ciencias.com/en/regulations/instructions/

\section{INDEXING IN:}

Emerging Source Citation Index

Fuente Academia Plus

DOAJ

Dialnet

\section{EVALUATED IN:}

MIAR: 9.3

ERIHPlus

Google Scholar Metrics: índex h5 $=3$ 


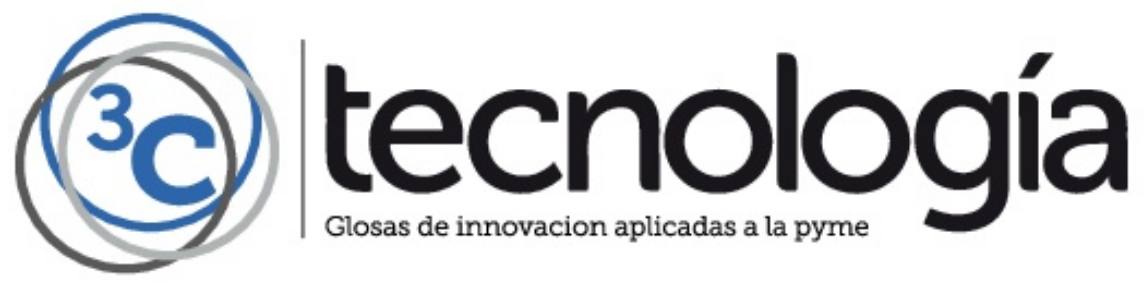


DEGRADATION OF MAGNETIC PROPERTIES OF NON-ORIENTED SILICON IRON SHEETS DUE TO DIFFERENT CUTTING TECHNOLOGIES 1

MAKING SELF-HELP VIRTUAL REALITY EXPOSURE THERAPY ACCESSIBLE: HARDWARE AND DESIGN CONSIDERATIONS 11

MACRO-ECONOMIC DETERMINANT AND INTERDEPENDENCE OF THE STOCK MARKETS: EVIDENCE FROM EMERGING ECONOMIES

ON CONVERGENCE OF ITERATIVE METHOD FOR DETERMINATION OF WEIBULL PARAMETERS BY MAXIMUM LIKELIHOOD METHOD 35

AUTO SPRUCE TRIAL SYSTEM (ASTS) 43

TOOL PATH OPTIMIZATION OF A 3D PRINTER VIA AN ENHANCED ELECTROMAGNETISM-LIKE MECHANISM ALGORITHM FOR SOLAR PANEL BRACKETS FABRICATION

ANALYZING SITE SUITABILITY FOR SOLID WASTE DISPOSAL THROUGH GIS MULTI-CRITERIA DECISION MAKING HIERARCHY PROCESS.

EFFECTING FACTORS OF KNOWLEDGE INTEGRATION THROUGH SOCIAL MEDIA IN SMALL MEDIUM ENTERPRISES ENVIRONMENT ....

AN OPTIMIZED HYBRID BEAMFORMING FOR MILLIMETER WAVE MUMASSIVE MIMO SYSTEM

A REVIEW ON ANTECEDENTS OF CITIZEN'S TRUST IN GOVERNMENT SOCIAL MEDIA SERVICES

SIMULATIVE INVESTIGATION OF TRANSMISSION PERFORMANCE FOR PROPOSED NG-PON 2 BASED ON DPQSK MODULATED DOWNSTREAM WITH TWO DIFFERENT INTENSITY MODULATED UPSTREAMS 121

EFFICIENT ENERGY UTILIZATION IN WIRELESS SENSOR NETWORKS: AN ALGORITHM

DESIGN AND IMPLEMENTATION OF TCSC FOR 500KV POWER TRANSMISSION SYSTEM.

DESIGN AND SIMULATION OF MEMS SHUNT CAPACITIVE SWITCH FOR LOWER SWITCHING TIME.

AUTOMERLIN MOBILE ROBOT'S BILATERAL TELECONTROL WITH RANDOM DELAY . 



\title{
DEGRADATION OF MAGNETIC PROPERTIES OF NON-ORIENTED SILICON IRON SHEETS DUE TO DIFFERENT CUTTING TECHNOLOGIES
}

\author{
Veronica Manescu (Paltanea) \\ University Politehnica of Bucharest, (Romania) \\ E-mail:
}

Gheorghe Paltanea

University Politehnica of Bucharest, (Romania)

E-mail:paltanea03@yahoo.com

Dorina Popovici

University Politehnica of Bucharest, (Romania)

E-mail: karina.popovici@yahoo.com

Gabriel Jiga

University Politehnica of Bucharest, (Romania)

E-mail: gabijiga@yahoo.com 


\section{ABSTRACT}

The magnetic properties of non-oriented silicon iron alloys are strongly influenced by the cutting technology. Medium quality electrical steel M800-65A samples were cut through mechanical punching, laser, water jet and electro erosion technologies and were characterized with an industrial Single Strip Tester at the peak magnetic polarizations Jp of $0.5,1$ and $1.5 \mathrm{~T}$, in the frequency range starting from $10 \mathrm{~Hz}$ to $200 \mathrm{~Hz}$. The influence of the cutting technology on the energy losses and magnetic permeability was investigated.

\section{KEYWORDS}

Non-oriented electrical alloys, Cutting technology, Energy losses, Relative magnetic permeability.

\section{INTRODUCTION}

Non-oriented electrical steels have a crystalline texture, which is characterized through a very low magneto-crystalline anisotropy. They are intensively used in the manufacture of the high efficiency electrical machines, in order to save energy and to avoid the overheating phenomenon. It is well known that the electrical machines have an energy consumption of 50\% from the worldwide electricity consumption and almost $6040 \mathrm{Mt}$ of $\mathrm{CO} 2$ emissions are due to these devices. According to the newest regulations, it is expected that in 2030, without proper environmental decisions, the energy consumption of the electrical motors will increase until 13360 TWh/year and the emissions will be equal to $8570 \mathrm{Mt} /$ year. Recently the energy losses of the newly produced electrical machines have been decreased with an amount of $20 \%$, by comparing them with those, measured before 2016 . The most important machine producers made motors in classes of premium efficiency (IE3) and above, i.g. Super Premium Efficiency (IE4) and Ultra Premium Efficiency (IE5) $[1,2,3]$. Nowadays it is a great challenge to produce an efficient electric motor, because thermal stresses, insulators' aging and low energy losses have to be taken into account. The most efficient motors are based on rare earth permanent magnets, but copper rotor electrical machines and synchronous reluctance motors could have comparable efficiency standards. Alternative current motors are designed to work at a constant speed, and its electronic drives improve the electrical machine flexibility. When a variable speed drive (VSD) is used, the inrush currents are decreased, the power factor has a good value, the effects of torque variation and speed drop are eliminated. In order to increase the efficiency of the electric motor, good quality of non-oriented materials, with low energy losses should be used $[4,5]$. Usually the magnetic cores of the electrical machines are prepared, by cutting the non-oriented alloys through mechanical punching, because this method is very fast and cheap, although it generates inside the material mechanical stresses that affect the energy losses and the magnetic properties. A work hardening phenomenon is expected to appear at the cut edge and deformation of the crystalline grains is present. Usually during a recrystallization process, these deformed grains are entirely transformed in new magnetic grains with better properties. The most important parameters of the cutting procedure are the clearance and the cutting angle, but also the hardness of the blade it is usually taken into consideration. When it is chosen a proper value of the clearance the induced mechanical stresses are minimal, and this fact contributes to the life increase of the cutting shears. A good quality cut edge is obtained, based on empirical determination for each cutting 
machine. When a strip is cut, only a small part is cut, and the rest is separated through fracture phenomenon [5]. The samples involved, in the paper were prepared, using a classical Computer Numerical Control (CNC) Turret punching machine. This device has a 3 axis Siemens special CNC system and uses AC servomotors. Its main components are actioned pneumatically, and it is equipped with concentrated lubricating systems, which decrease the friction during the cutting procedure.

Sometimes the electrical machines' producers want to obtain a free burr cut edge and they choose the non-conventional cutting technologies as laser. Laser method is a very flexible one, but unfortunately it induces important thermal stresses and the method is expensive. In the case of $\mathrm{CO} 2$ laser, the single generated wavelength is in Infra-Red spectrum. The beam has an $0.025 \mathrm{~mm}$ diameter, when it travels from the laser resonator to the beam path. It is guided through a mirror or a special lens' system and finally is focused on the material. The laser beam is accompanied by a compressed gas, as Oxygen or Nitrogen. The cut edge is almost perfect, but the high power density of the beam has an unwanted result, which consists of a rapid heating, melting or partial vaporizing of the material. In the paper a Morn Laser machine was used to cut the samples. This machine is a very performant one and the laser cut has no cracks or supplementary deformation due to the thermal stresses and it has a very high stability of the cutting tool $[6,7]$.

Other non-conventional cutting technologies are the electro-erosion and water jet methods. The electro-erosion (EDM) produces any stresses, but the process is very slow, and it can be used only for small dimension electrical machines. Today EDM machines are very stable, starting with 1980 due to the introduction of the CNC in the EDM technology and they could be used to cut complex shapes. The basic phenomenon involved in the EDM cutting procedure is the energy transformation from electrical into thermal energy, using a series of electrical discharges that appear between an electrode and a workpiece, introduced in a dielectric fluid. This fluid is a very special one, because it has to avoid the electrode electrolysis. The EDM procedure is a modern technique, based on material erosion, when a spark appears as a result of an applied voltage between the electrode and the material surface. The dielectric fluid is a cooler medium and it helps the discharge energy to be concentrated on a small area. As the erosion advances, the electrode is moved through the dielectric fluid. Recently, servo systems are used, to assure a constant gap voltage, between the material and the wire and to retract the electrode, when a short circuit occurs [5, 6, 7, 8]. A Kingred Wire EDM, controlled by a CNC system, which utilizes brass electrode wire and high frequency impulses, was used. This machine is very suitable for cutting materials with high precision [9].

The water-jet leads also to a very good quality of the cut edge, but special expensive equipment is needed, and the cutting speed is relatively slow. Abrasive particles as Garnets are used and the price of the cutting procedure is very high. The abrasive particles do the material cut through a sawing action and it leaves a precision cut surface. This method is suitable to cut almost any type of steels and it has a narrow kerf width. Physical properties such as melting point, thermal and electric conductivity, density have a limited importance, although the hardness of the material could reduce the cutting speed. This process induces no heat affected zone 
and no hard oxidation layers on the cut edges, which could lead to the microcracks apparition. This technology damages minimal the magnetic properties of the material due to the plastic deformation. An Omax Waterjet machine was used. This device performs a very high accurate edge cut and it uses Garnet as abrasive particles.

The non-conventional methods are adequate in the prototyping production.

\section{MATERIALS AND METHODS}

A medium quality commercial non-oriented steel M800-65A was investigated. The material properties and the geometrical parameters are shown in Table 1.

Table 1. Properties and geometrical parameters of the M800-65A samples.

\begin{tabular}{|l|l|l|l|l|l|l|l|}
\hline Material & $\begin{array}{l}\text { Cut } \\
\text { direction }\end{array}$ & $\begin{array}{l}\text { Density } \\
{\left[\mathrm{g} / \mathrm{cm}^{3}\right]}\end{array}$ & $\begin{array}{l}\text { Electrical } \\
\text { resistivity } \\
{[\Omega / \mathrm{m}]}\end{array}$ & $\begin{array}{l}\text { Mass } \\
{[\mathrm{g}]}\end{array}$ & $\begin{array}{l}\text { Length } \\
{[\mathrm{mm}]}\end{array}$ & $\begin{array}{l}\text { Width } \\
{[\mathrm{mm}]}\end{array}$ & $\begin{array}{l}\text { Thickness } \\
{[\mathrm{mm}]}\end{array}$ \\
\hline M800-65A & $\begin{array}{l}\text { Rolling } \\
\text { direction }\end{array}$ & 7.80 & $25 \times 10^{-8}$ & 44.73 & 300 & 30 & 0.65 \\
\hline
\end{tabular}

The influence of the cutting procedure on the energy losses was analyzed using the energy loss separation method. According to this theory, the total energy losses are divided into hysteresis, classical (Foucault) and excess energy losses.

The hysteresis losses are due to the pinning points and impurities that are present in the medium quality non-oriented steels and they are usually analyzed, by taking into account the coercivity mechanisms. They can be computed, by extrapolating in zero the measured total energy losses.

The classical energy losses are generated by the eddy currents and the material is treated as a homogenous medium [10]. They can be computed with the following equation:

$$
W_{c l}=\frac{\pi^{2} \sigma J_{p}^{2} d^{2}}{6 \rho} f,
$$

where $d$ is the sample thickness, $\sigma$ is the electrical conductivity, $\rho$ is the non-oriented steel density and $f$ is the experimental frequency.

The excess losses are due to the micro eddy currents, which are formed in the vicinity of the domain walls. They are computed, subtracting the classical and hysteresis losses from the total energy losses.

The magnetic measurements were done, by using an industrial Brockhaus Single Strip tester, with a double $\mathrm{C}$ yoke, which is a standardized device and it controls at each step of the measurement the form of the secondary voltage, in order to be a sinusoidal one according to DIN 50462 standard. This device permits the measurement of the energy losses and of the relative magnetic permeability with its components: real and imaginary parts. An external magnetic field is applied, and a magnetic flux is generated into the tested sample. The current, in the magnetizing coil, is determined with the help of a shunt resistor. The magnetic polarization is computed, by integrating the experimentally induced voltage on the measuring coil 
with a 16-bit processor. The accuracy of the measurements is very high, and the device provide a $0.2 \%$ repeatability of the results. The maximum current is equal to $5 \mathrm{~A}$ and the maximum voltage is set at $32 \mathrm{~V}$. The magnetic path length, between the polar pieces is $240 \mathrm{~mm}$. Along this path are placed the measuring (723 windings) and the magnetizing (704 windings) coils. A sample of minimum $280 \mathrm{~mm}$ length and maximum $30 \mathrm{~mm}$ width could be investigated.

\section{RESULTS AND DISCUSSIONS}

Samples of medium quality commercial electrical steel of M800-65A grade were cut through punching, laser, water-jet and electro-erosion technologies.

The normal magnetization curve, defined as the geometrical place of the symmetrical hysteresis cycle peak points, which extends from the demagnetized state to the saturation is presented in Figure 1. The demagnetized state could be obtained, by increasing the sample temperature to a value, higher than the Curie temperature, followed by a normal cooling, in the absence of a magnetic field. Another technique consists of applying an alternative magnetic field, whose amplitude is progressively decreased through zero, starting from a higher reference value, which implies the technical saturation point. After the material demagnetization, if a monotone magnetic field is applied, the sample behavior follows the virgin magnetization curve. If this procedure is done after the cyclic demagnetization of the material, the normal magnetization curve is obtained. In the soft magnetic material case there are some minor differences between these two curves and also the magnetic polarization $\mathrm{J}$ is considered to be equal to the magnetic flux density B [1].

In order to experimentally determine the normal magnetization curves of the samples, measurements were done at the industrial frequency of $50 \mathrm{~Hz}$. In the case of each sample, symmetrical hysteresis loops were determined at a peak magnetic polarization Jp of $5 \mathrm{mT}, 10 \mathrm{mT}, 20 \mathrm{mT}, 50 \mathrm{mT}, 100 \mathrm{mT}, 200 \mathrm{mT}, 500 \mathrm{mT}, 750 \mathrm{mT}$, $900 \mathrm{mT}, 1000 \mathrm{mT}, 1100 \mathrm{mT}, 1200 \mathrm{mT}, 1300 \mathrm{mT}, 1400 \mathrm{mT}, 1500 \mathrm{mT}, 1600 \mathrm{mT}$. It can be noticed from Figure 1 that the electro-erosion and water jet technologies determines an easier magnetization of the material, because the cutting procedure induces any thermal or mechanical stresses. The punching and the laser procedures leads to a more difficult magnetization process due to the generated stresses, although the M800-65A grade is an alloy, which contains a relative high percent of non-magnetic impurities that acts as pinning points for the magnetic domain wall movement. All the normal magnetization curves meet at the saturation point of the material, for a magnetic field strength of $2000 \mathrm{~A} / \mathrm{m}$. The principal magnetization process in this type of steel is the reversible domain wall movement and near the saturation zone rotating of the spin magnetic moments occur.

In Figure 2 is presented the variation of the total energy losses as a function of the frequency, for three values of the peak magnetic polarization Jp of $500 \mathrm{mT}, 1000$ $\mathrm{mT}$ and $1500 \mathrm{mT}$. At $500 \mathrm{mT}$ the water-jet technology leads to the lowest value of the total energy losses, followed by the punching method and the highest energy losses are measured in the case of laser. These observations are valid in the case of $1000 \mathrm{mT}$, but for $1500 \mathrm{mT}$ the lowest value of the energy losses is determined for the electro-erosion technology. It can be noticed that with increase of the peak 
magnetic polarization, the influence of the cutting technology on the energy losses is reduced.

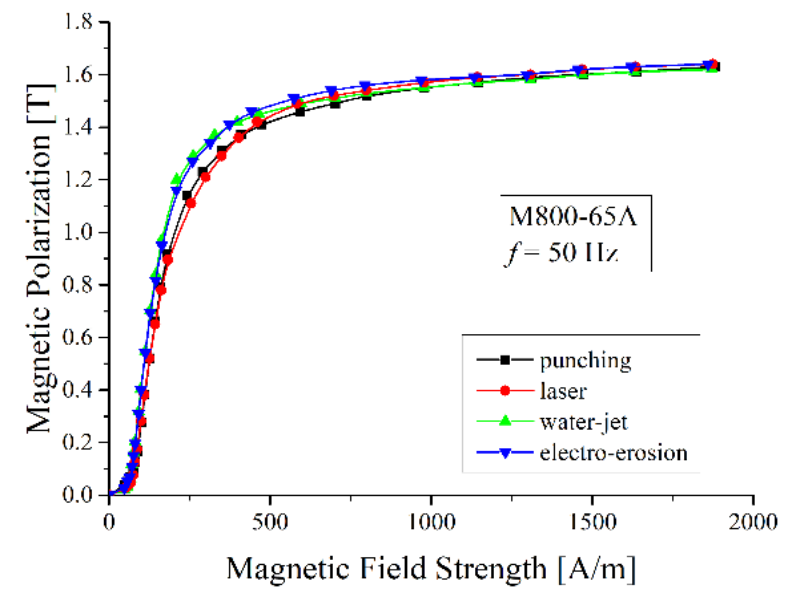

Figure 1. Normal magnetization curve for M800-65A samples, cut through punching, laser, waterjet and electro-erosion.

The hysteresis energy losses are invariable with the frequency and they are a direct consequence of the magnetization processes, which are due to the magnetic domain wall movements.

It can be observed from Figure 3 that the most important variation of the hysteresis energy losses is noticed for the high magnetic polarization domain, especially in the case of punching and laser. The lowest values of the hysteresis energy losses are determined for the electro-erosion and water-jet cutting technologies.

The classical energy losses are generated by the eddy currents and they are directly proportional with the peak magnetic polarization and the frequency.

It can be noticed that from a specific value of the frequency these losses become predominant with higher values than in the case of excess and hysteresis energy losses.

In Figure 4 is presented the variation of the classical energy losses with the frequency.

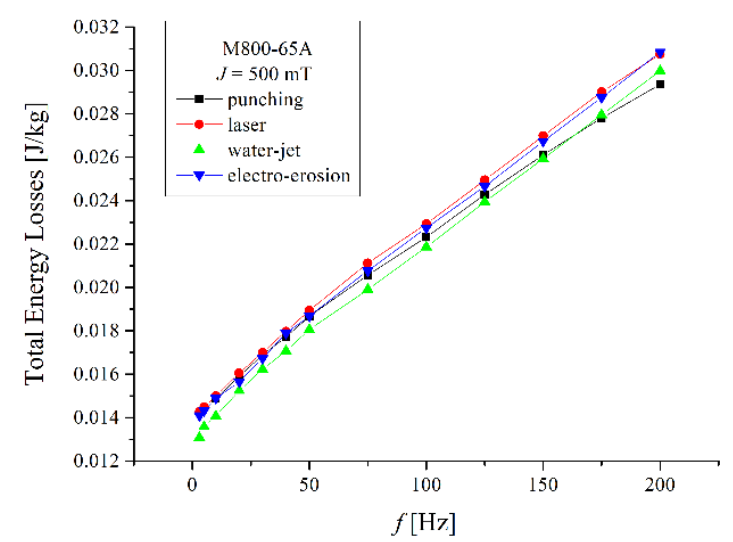

a)

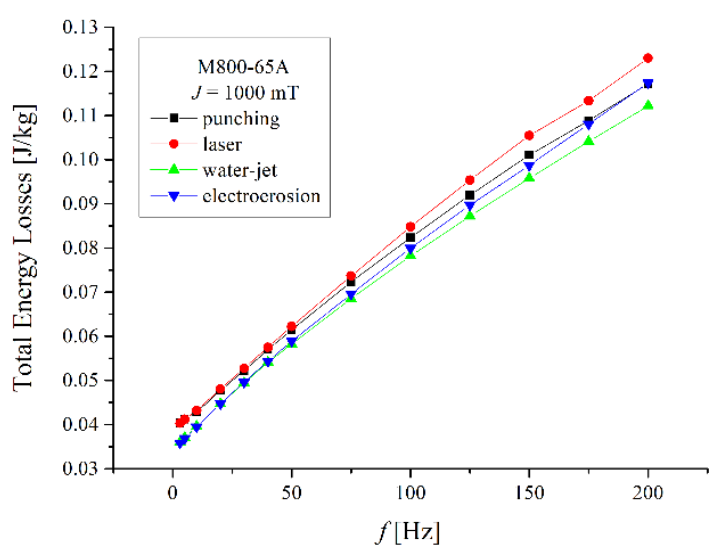

b) 


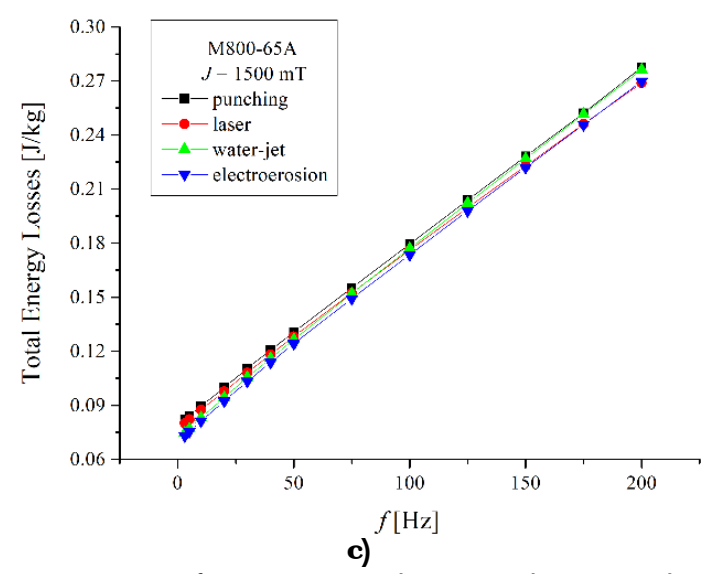

Figure 2. Total energy losses versus frequency at three peak magnetic polarizations of $500 \mathrm{mT}$ (a), $1000 \mathrm{mT}$ (b) and $1500 \mathrm{mT}$ (c).

The excess energy losses are influenced by the cutting procedures in the case of all the peak magnetic polarization values. The water-jet technology leads to the lowest value, followed by the laser and the electro-erosion procedures.

The mechanical punching has a strong influence on the magnetic domain structure and is directly linked to the existence of higher values of the excess energy losses.

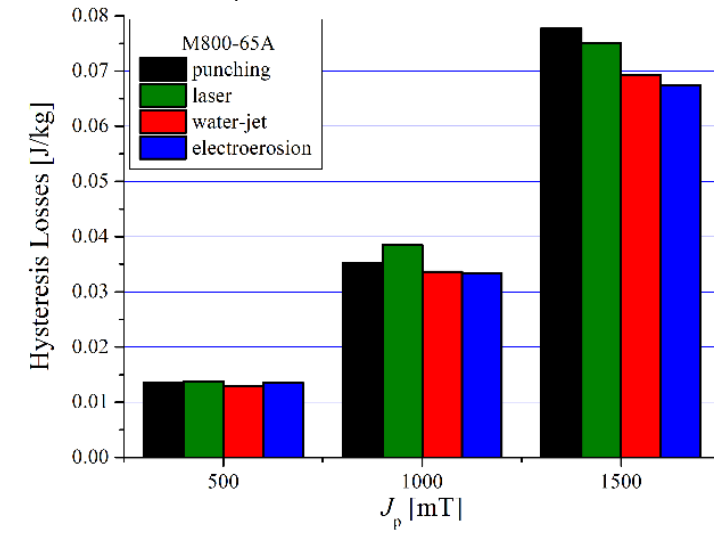

Figure 3. Hysteresis energy losses versus peak magnetic polarization, in the case of different cutting technologies.

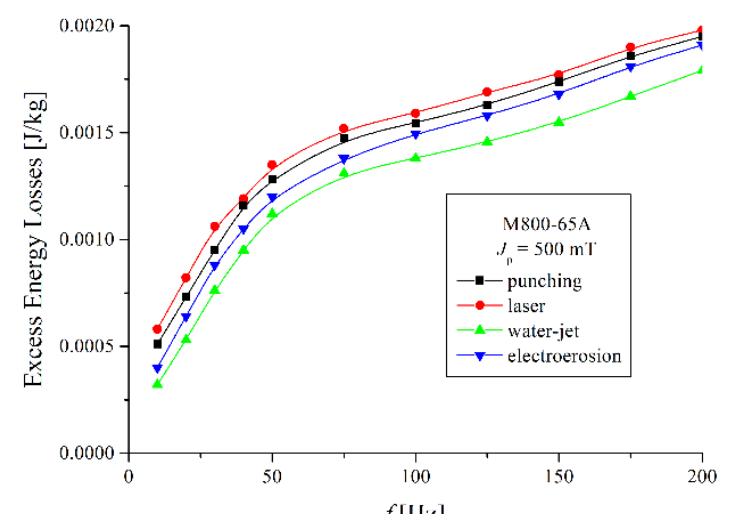

a)

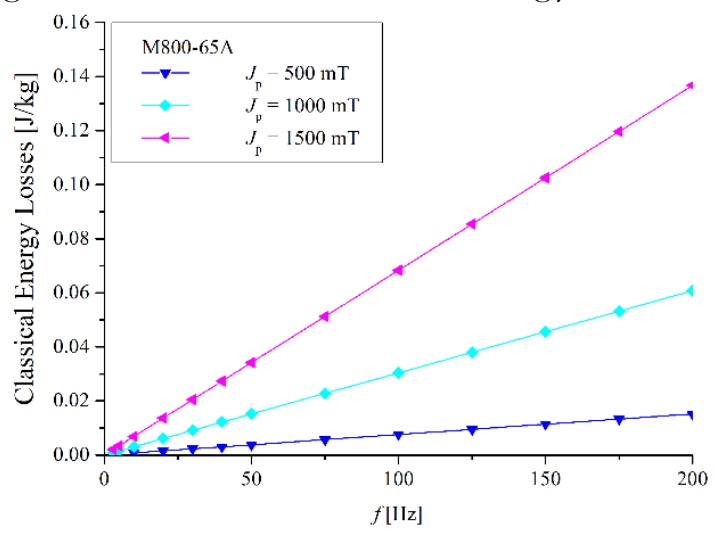

Figure 4. Classical energy losses versus frequency at three peak magnetic polarizations of $500 \mathrm{mT}, 1000 \mathrm{mT}$ and $1500 \mathrm{mT}$.

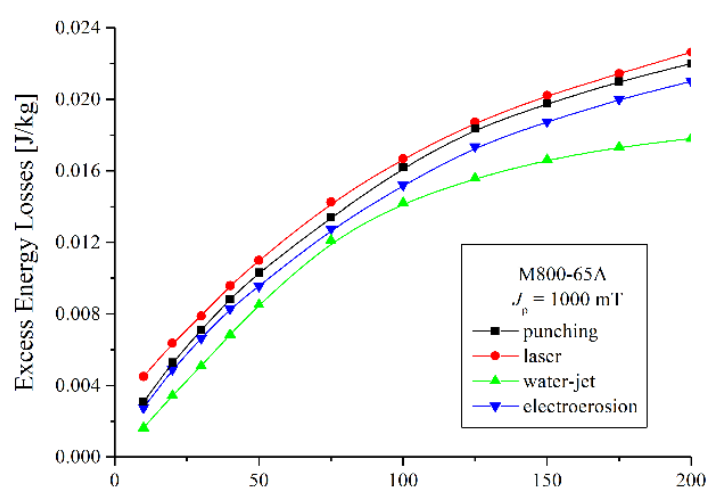

b) 


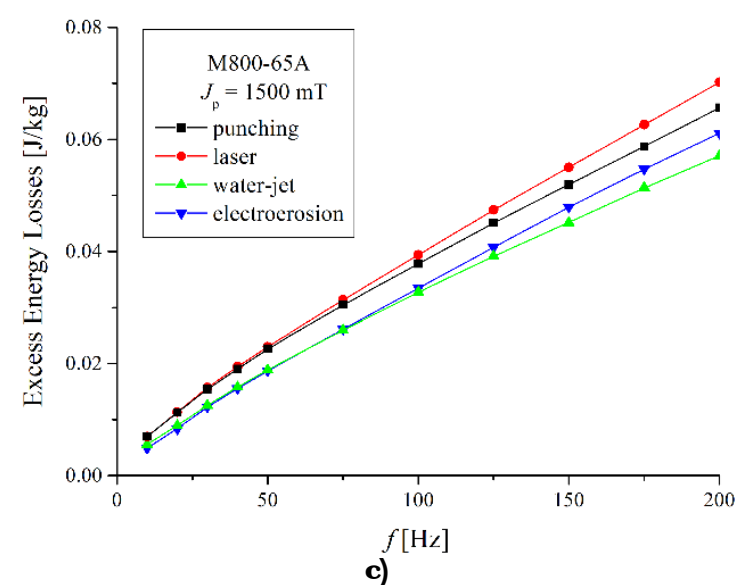

Figure 5. Excess energy losses versus frequency at three peak magnetic polarizations of 500 $\mathrm{mT}(\mathrm{a}), 1000 \mathrm{mT}(\mathrm{b})$ and $1500 \mathrm{mT}$ (c).

The relative magnetic permeability $\mu_{\mathrm{r}}$ is a physical quantity, which describes the material property to concentrate the magnetic field lines, when an external magnetic field is applied. A magnetic material is more adequate to be used in industrial applications in the case of high values of magnetic permeability. In Figure 6 . is presented the variation of the relative magnetic permeability for two peak magnetic polarization of $500 \mathrm{mT}$ and $1000 \mathrm{mT}$. It can be noticed that the magnetic permeability presents an inversely proportional variation with the frequency. The influence of the cutting procedure is more pronounced for frequency values lower than $100 \mathrm{~Hz}$. The highest value of the magnetic permeability is obtained for the water-jet and electro-erosion technologies.

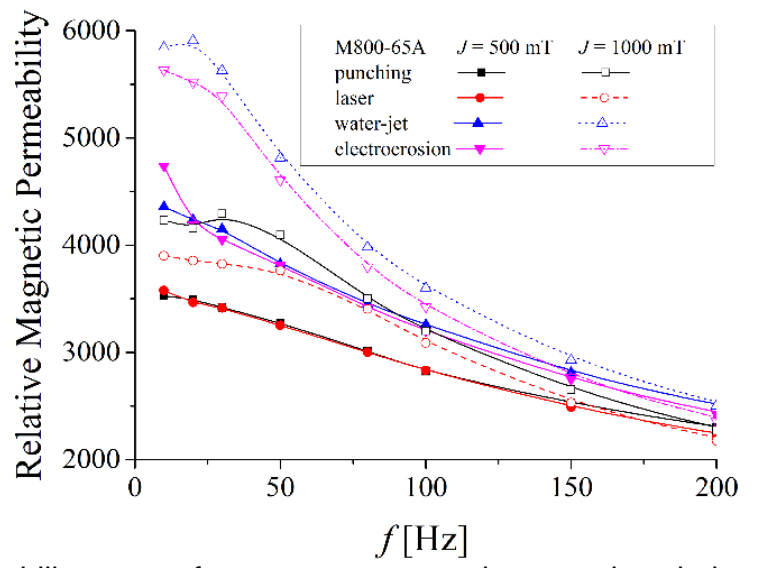

Figure 6. Magnetic permeability versus frequency at two peak magnetic polarizations of $500 \mathrm{mT}$ and 1000 $\mathrm{mT}$, in the case of different cutting technologies.

\section{CONCLUSIONS}

The cutting procedure damages the magnetic material microstructure, more pronounced in the case of laser, followed by punching, electro-erosion and waterjet. To reclaim the initial magnetic properties of the alloy some thermal recrystallization treatments are required, but this step is not taken into consideration by the electric motor manufacturers, because it damages the insulator layer that covers the magnetic core sheets. The use of water-jet or electro-erosion technologies that have a reduced impact on the energy losses and the relative permeability is taken into consideration only in the prototyping and special cases, because of their slow cutting speed. As a compromise between cutting speed and the 
induced damage on the magnetic properties the electrical motor manufacturers still prefer the classical mechanical punching.

\section{ACKNOWLEDGEMENTS}

The work of Veronica Mănescu (Păltânea) has been funded by University Politehnica of Bucharest, through "Excellence Research Grants" Program, UPBGEX 2017. Identifier: UPB-GEX2017, Ctr. No. 04/25.09.2017 (OPTIM-IE4). The work of Gheorghe Paltanea has been funded by University Politehnica of Bucharest, through "Excellence Research Grants" Program, UPB-GEX 2017. Identifier: UPBGEX2017, Ctr. No. 02/25.09.2017 (ANIZ-GO).

\section{REFERENCES}

[1] G. Bertotti, Hysteresis in Magnetism, San Diego, CA: Academic Press, (1998).

[2] V. Mănescu (Păltânea), G. Paltanea and H. Gavrilă, Physica B, 486, (2016).

[3] A.T. De Almeida, F. Ferreira, J. Fong, B. Conrad, Electric Motor Ecodesign and Global Market Transformation, Proceedings of IEEE Industrial \& Commercial Power Systems Conf., (2008), May, Florida, USA.

[4] M Enokizono, IEEE Trans. Magn., 48, 11, (2012).

[5] V. Manescu (Paltanea), G. Paltanea, H. Gavrila, G. Scutaru, Rev. Roum. Sci. Techn.-Electrotechn. Et Energ., 60, 1, (2015).

[6] V. Manescu (Paltanea), G. Paltanea, H. Gavrila, Rev. Roum. Sci. Techn.Electrotechn. Et Energ., 59, 4, (2014).

[7] O.S. Bursi, M. D’Incau, G. Zanon, S. Raso, P. Scardi, JCSR, 133 (2017).

[8] B. Boswell, M.N. Islam, I.J. Davies, Int. J. Adv. Manuf. Technol., (2017).

[9] V. Manescu (Paltanea), G. Paltanea, H. Gavrila, A. Nicolaide, Rev. Roum. Sci. Techn.-Electrotechn. Et Energ., 60, 2, (2015).

[10] M. Stanculescu, O. Drosu, M. Maricaru, Reduction of winding losses for trapezoidal periodic currents, Proceedings of IEEE $8^{\text {th }}$ International Symposium on Advanced Topics in Electrical Engineering, (2013), May, Bucharest, Romania.

[11] G. Paltanea, V. Manescu (Paltanea), , H. Gavrila, D. Popovici, Magnetic property analysis in non-oriented silicon iron steels cut through water jet technology, 2016 ISFEE.

[12] D. Popovici, F. Constantinescu, M. Maricaru, Modeling and Simulation of Piezoelectric Devices, June 2008, book: Modelling and Simulation, Vienna, Austria, ISBN 973-8067-96-0. 


\section{AUTHORS}

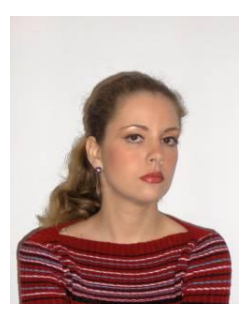

\section{Veronica Mănescu (Păltânea)}

Veronica Mănescu (Păltânea) was born in Bucharest, Romania, on June 5, 1978. She received the B.E. degree in electrical engineering from the Politehnica University of Bucharest in 2002, and the M.S. and Ph.D. degrees in electrical engineering from the Politehnica University of Bucharest, Romania, in 2004 and 2008, respectively. She is actually an Associate Professor at U.P.B.

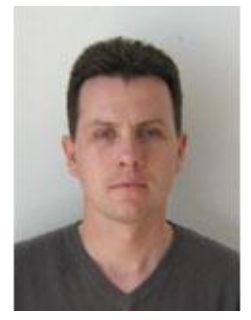

\section{Gheorghe Păltânea}

Gheorghe Păltânea was born in Bucharest, Romania, on November 3, 1978. He received the B.E. degree in electrical engineering from the Politehnica University of Bucharest, in 2002, and the M.S. and Ph.D. degrees in electrical engineering from the Politehnica University of Bucharest, Romania, in 2004 and 2008, respectively. He is actually an Associate Professor at U.P.B.

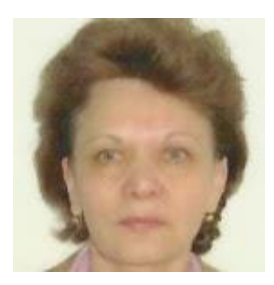

\section{Dorina Popovici}

Dorina Popovici received in 1989 the Ph.D. degrees in electrical engineering from the Politehnica University of Bucharest, Romania. She is the author of over 100 scientific articles from which over 80 international journals, conferences, symposiums and workshops published 15 courses and applications books and participated as project manager in over 23 national and international research projects.

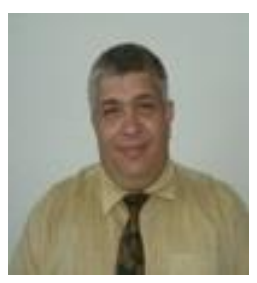

\section{Gabriel Jiga}

Gabriel Jiga received in 1996 the Ph.D. degree in civil engineering at the Technical Military Academy, Romania with a subject in structural analysis of composite structures. He is the author of over 120 scientific papers, more than 80 being presented at international conferences and symposia or published in prestigious international journals. Nowadays he is full professor at University Politehnica of Bucharest and teach 


\title{
MAKING SELF-HELP VIRTUAL REALITY EXPOSURE THERAPY ACCESSIBLE: HARDWARE AND DESIGN CONSIDERATIONS
}

\author{
Muhammad Tahir \\ Department of Computer Engineering \\ Sir Syed University of Engineering and Technology, Karachi, (Pakistan) \\ E-mail:tahirfattani@gmail.com
}

Rabia Noor Enam

Department of Computer Engineering Sir Syed University of Engineering and Technology, Karachi, (Pakistan)

E-mail: afaq_rabia@yahoo.com

Najma Ismat

Department of Computer Engineering

Sir Syed University of Engineering and Technology, Karachi, (Pakistan)

E-mail: najma_ismat@yahoo.com

Syeda Fizza

Department of Computer Engineering

Sir Syed University of Engineering and Technology, Karachi, (Pakistan)

E-mail:fizzy.syeda@gmail.com 


\section{ABSTRACT}

Virtual Reality Exposure therapy has proven to be as effective as cognitive behavioral therapy with in vivo exposure [1]. Since it is an efficacious and cost effective alternative to in vivo exposure therapy, it can be offered to the clients via software packages containing educational material and self- report questionnaires considering the present state of Virtual Reality technology. This paper aims to identify design considerations and constraints, knowledge of which is highly useful in developing the applications that are accessible user-friendly, require minimal or no therapeutic contact and provide self-help solution to the inaccessibility of anxiety treatment which leads to a massive number of patients being left untreated.

\section{KEYWORDS}

Anxiety, Phobia, Virtual Reality, Exposure Therapy, Vivo Exposure.

\section{INTRODUCTION}

Anxiety disorder is the most prevalent mental disorder. But the stigma attached to mental disorders in addition to the unavailability of proper and affordable treatment leads to it being left untreated. Virtual Reality Exposure Therapy is being studied for a long period of time and can be considered an effective alternative to cognitive behavioral therapy [2]. The major barrier in receiving any kind of treatment is the lack of its accessibility and affordability. According to a psychiatry journal, people generally seek treatment after 6 to 8 years of suffering from mood disorders and 9 to 23 years of suffering from anxiety disorders [3]. With the rapid growth of interest in VR technology, the future of VRET is promising.

As VRET becomes convenient, it will be easier to develop standalone VRET experiences but it requires consideration of design and hardware. Developing such virtual environments not only requires understanding of human side of VR but also the understanding of the specific phobias and disorders the environment is being designed. This will make VRET applications efficacious, affordable and accessible which will lead to patients seeking help leading to less delay in initial contact for treatment and delay in treatment of the disorder.

\section{VRET AND ACCESSIBILITY}

The common question asked regarding VRET is that why VRET is considered if the option in vivo exposure therapy is available. In vivo exposure therapy with or without relaxation is just as effective but VRET is a good alternative if not better for the following reasons:

- VRET allows the user to have more control over the situation [4]. The application that is designed a way in which the exposure is increased by using various levels and after taking subjective units of discomfort (SUDs) in account will less reluctance from patient.

- In vivo exposure can be expensive when it comes to exposing patient to environments that are usually out of reach. For instance, if patient had flight phobia, in vivo exposure will be costly [5]. There is a high chance of fear coming back after just one session. This virtually generated environment will be an aid is this situation or in a similar one.

- Refusal to initiate a treatment is a major issue when it comes to in vivo exposure. According to a survey regarding the acceptability of virtual reality 
(VR) exposure, the refusal rate of initiating treatment with in vivo exposure was greater than VR exposure. Thus it can be concluded that VRET results in more people seeking therapy [6].

- Another major hindrance in seeking help is confidentiality. People like to keep the information regarding their treatment confidential. According to an article published in Journal of Health Cognitive Behaviour Therapy 2017 [7], "An ethical advantage of VRET is that you can do "in-vivo"-like exposures without leaving the office."

\section{DESIGNING VIRTUAL ENVIRONMENT FOR THERAPY}

For designing virtual environments, many considerations are to be made. Few of them are listed thoroughly below:

\subsection{Hardware}

\section{i. Terminology}

- Head Mounted Display (HMD): A head-mounted display is worn over head. For VR, stereo binocular HMDs are used which means that two separate images are rendered, one for each eye. In short it us the combination of two monocular displays.

- Virtual Environment: Virtual environment is anything that is visible to the user when he puts on head mounted display. Virtual environment stimulates mind by presenting it visuals and sometimes makes use of other senses.

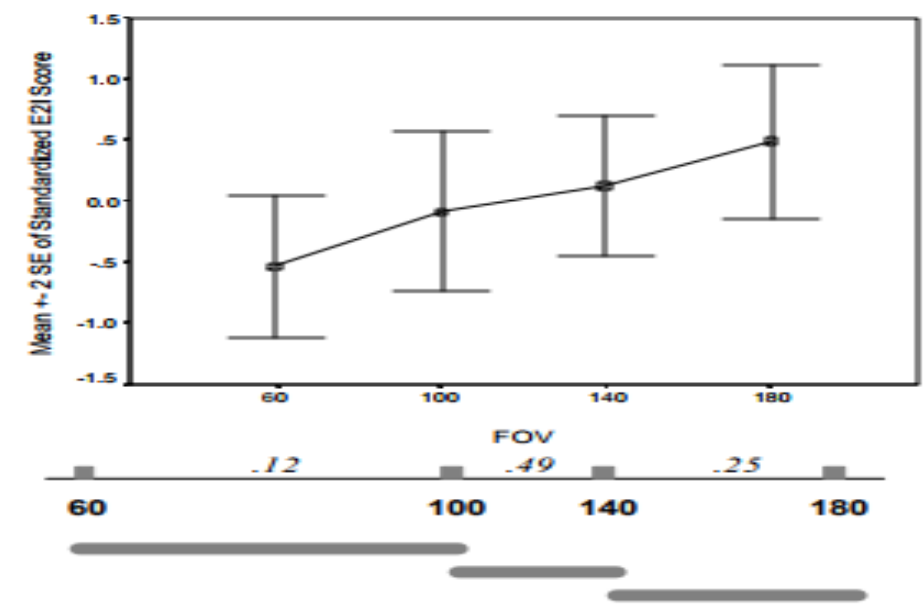

Figure 1. Mean and standard error of $E^{2}$ I scores [8].

- Simulator Sickness: Motion sickness that maybe induced by exposure to virtual environments due to badly designed experience, too much movement or prolonged use.

- Immersion: Immersion is the measure of presence user feels in the virtual environment.

- Degree of Freedom (DOF): Degree of freedom is the level of dependency the hardware allows for translation and rotation in the virtual environment.

- Field of View (FOV): It is the space that is visible to user at any given time in virtual reality. A research verified the relation between engagement, enjoyment and immersion graph and the field of view 
[8]. The results are illustrated in the graph which is shown in figure [1]:

\section{ii. Past Limitations of Hardware}

VRET proved to be efficacious in the past but there were many hardware limitations present. It is important to specify those limitations to assist in finding the appropriate HMD for own design.

These limitations are listed below:

- Low computational Power: In the past, head mounted displayed had low computational power which has improved drastically with the advancement of VR technology.

- Low Frame Rates: Maintaining frame rate of over 75 frames per second is very important for a good virtual reality experience. With new HMDS, it is now possible to maintain that frame rate. Low frame rate also cause nausea, bad user experience and distortion of view which results in breaking of immersion. The inconsistencies in the motion of simulator and the user cause simulator sickness [9].

- Low Resolution: Since in VR, images are not viewed on one single flat panel screen, instead they are blown up. Low resolution results in making experience less immersive.

- Low Refresh Rate: Refresh rate is another factor that can potentially contribute to simulator sickness. It is the rate at which the content on the screen are updated. It is measured in Hertz. It is important to have a refresh rate of $90 \mathrm{~Hz}$ in VR. It is possible to have a refresh rate of $120 \mathrm{~Hz}$ with some of the current HMDs which was difficult to achieve in the past.

- Inaccessibility: HMDs are now available easily at different prices in the market. This was not the case in the past. It was difficult to find a VR system and set it up for proper use.

- Installation: Installation and setup of VR devices required a lot of expertise. Now it can all be done with few clicks.

These limitations were stated above in order to describe the parameters that should be considered when choosing hardware for VRET and they can be summarized as in Table 1:

Table 1. Summarized Specifications.

\begin{tabular}{|c|c|c|}
\hline 1 & Frame Rate Per Second (FPS) & $>60 \mathrm{fps}$ \\
\hline 2 & Refresh Rate & At least $60 \mathrm{~Hz}$ \\
\hline 3 & Resolution & Varies with HMDs \\
\hline 4 & Field of View & Varies with HMDs \\
\hline
\end{tabular}

\section{iii. Other Considerations}

Another important question to ask is whether the platform is a mobile platform or stationary VR. With 3 DOFs, head rotation is tracked by HMDs which is called orientation tracking and with 6 DOFs, head rotation with translation and movement in spaced is tracked which is called positional tracking. The hardware should be chosen considering all these parameters. This choice depends on the target 
application and environment design. There are few limitations of mobile VR, for instance, computational power is low, and fewer head-tracking and user input options are available. Stationary VR systems cost more and allows user to share the experience. This advantage may help the therapist to get involved in the experience with the user.

Table 2. A Comparison of VR Platforms for VRET.

\begin{tabular}{|c|c|c|}
\hline Platforms & Stationary & Mobile \\
\hline $\begin{array}{c}\text { Cont } \\
\text { by Therapist }\end{array}$ & High Cost & $\begin{array}{c}\text { Only through verbal } \\
\text { communication }\end{array}$ \\
\hline $\begin{array}{c}\text { Controlling Environment by } \\
\text { Therapist }\end{array}$ & Easy & Possible \\
\hline $\begin{array}{c}\text { Computational Power } \\
\text { Appropriateness for Self-Help } \\
\text { VRET Applications }\end{array}$ & Possible & Low \\
\hline User Input Options & $\begin{array}{l}\text { More options available then } \\
\text { mobile VR }\end{array}$ & Limited \\
\hline
\end{tabular}

\subsection{Personal Presence and Movement}

Personal presence is the degree to which a user feels immersed in the virtual environment where as environmental presence is defined as the extent to which the environment reacts to the user's presence. Complex visuals improve the personal presence is VRET. A research done with regards to public speaking proved that user reacts more to animated public than to static public [10]. Thus a moving and interactive object or stimuli will immerse the user more than a static object.

Movements require more computational sources but in the long run it make the experience more effective. It is also discussed in a research that a realistically moving but graphically poor object is stimulates a user more than a realistically textured static object. High end graphics are not requires as long as anxiety provoking factor of motion is present [11]. Some textures in VR can also be computationally expensive. Thus, computational resources should be utilized carefully considering these results.

There is no research present that proves any effect of user movement in the virtual environments towards the stimuli. But if a virtual environments demands movement, it should be kept minimal as it may cause motion sickness.

\subsection{Sound}

Similar to the reality we live in, it is important in VR that sounds come from a proper source. This does not only help with interactivity but also helps in making sure that immersion never breaks at any point. It keeps the real world distractions away from the users. Few design decisions that you have to make regarding sound are:

- 2D or 3D Audio: 2D sounds are usually used for 2D screens and applications. If a VR experience has different sound sources, then $3 \mathrm{D}$ audio should be preferred. 
- Mono or Stereo: Stereo makes a user feel that he is located in a three dimensional sound source. Mono sounds save disk space but they are better suited for 2D designs.

- Binaural Sounds: 3D sounds can be used to draw a user's attentions. It is particularly important in a VR experience because user can look in any direction and the source of sound must make sense to the user.

Sounds in VRET are to be carefully picked. It is proven that stereo sounds are appropriate for VR experiences. According to a research, people can differentiate between stereo and mono sounds. This result had a great effect on the developed environment [12].

Another question that arises is if $3 \mathrm{D}$ audio has any relation with the presence in the environment. The results presented by research state that "significant difference in spatial perception was found between 3Daudio and stereo or Dolby sound, although the median score indicated a trend in favor of 3D audio" [12].

For VRET, a good design decision is to avoid using sounds that are not relevant to the environment and only to use sound that may enhance the experience. If a voice assistant is to be included, it is better to make sure that it's not adding to anxiety of the patient which may lead to patient dropping out of therapy.

Good sound decisions enhance the user's experience during VRET in following ways:

- For VRET, the exposure intensity is predominantly a measure of visuals cues but sound plays an important role in creating the illusion. For therapy to be effective, it is important to match audio with the visuals.

- If the stimulus is not chiefly visual, appropriate sound setup will increase efficacy.

- Voice assistance is an added advantage for blind and partially sighted patients [13].

- It helps in drawing attention of user to different spots within the scene. For instance, you may want the user to look down from a glass elevator for arachnophobia, thus to draw user's attention to the ground, an appropriate sound can be used.

- If mind-relaxation or other calming sounds are needed at some point in therapy, they should be played at the right moment for the right amount of time to avoid the mismatch of simulation and sound.

- Attaching 3D sounds to object is a technique that can be used through rendering engines which leads to all the sound coming from the right distance from the correct object. As the users distance from the object increased, the effect of sound decreases [14].

\subsection{Degree of Interactivity}

Interactivity plays a big role in increasing the presence hence improving the efficacy of VRET. The extent to which an environment can be made interactive is dependent on different variables. Some of them were elaborated in a research [15] and are listed below:

- Number of inputs that environment responds to.

- The extent to which the environment can be modified by the user.

- The extent to which environment alters during the experience. 
- Ability to realistically interact with objects in virtual environment, for instance, the ability to hold and throw object with hands rather than click of a button.

- The responsiveness speed when user interacts with the environment.

Increasing the number of inputs will not have any positive effect but interactivity should be added where it is required.

\subsection{User Interface}

Designing UI for a VR experience is a rather challenging task. There are all kinds of options available which will be stated for assistance. But it entirely depends on how the environment is setup. Design of user interface depends on many factors. A research summarized the guidelines and preference for therapist led sessions. These are summarized as [16]:

- Therapists should be provided an automated environment.

- Provide therapists with the input from the user including SUD scores, their comments, options they choose and their current status.

- Design for error prevention by not allowing therapists to trigger inappropriate simulation events.

- Provide therapists with predefined comment flags to record events in the session.

- In a VRET environment where the position of the patient is fixed in the VR world, therapists do not need an external viewpoint of the VR world with a projection.

For self-help applications or minimal therapist contact VRET these design considerations may still be helpful. In a VR environment, user should be able to interact easily; user should be able to control the stimuli while being encouraged to continue the therapy. Another alternative is to use Subjective Units of Distress (SUDs) to choose the level of exposure which is shown in table [3]. SUDs can be selfreported and recorded through user input.

Table 3. Subjective Units of Distress Scale.

\begin{tabular}{|c|l|}
\hline \multicolumn{1}{|c|}{ Score } & \multicolumn{1}{|c|}{ Intensity of Disturbance } \\
\hline 10 & Close to a nervous breakdown \\
\hline 9 & Losing Control \\
\hline 8 & Alienation \\
\hline 7 & Maintaining control with difficulty \\
\hline 6 & Moderately Uncomfortable \\
\hline 5 & Somewhat Uncomfortable \\
\hline 4 & Mildly Upset \\
\hline 3 & Feeling Unpleasant \\
\hline 2 & Bit Bothered \\
\hline 1 & No or Minor Distress \\
\hline 0 & Total Relief \\
\hline
\end{tabular}




\section{DESIGNING COMPONENTS}

\subsection{Progression through Levels}

In therapist-led applications, therapist is given a scene-control which they can adjust as they communicate verbally with patients. Some of the controls that patients and therapist possess are:

Table 4. Scenario Controls.

\begin{tabular}{|l|l|}
\hline Therapist's Control & Patient's Control \\
\hline Check for SUD's & Input SUDs \\
\hline Ask for SUD's Input & $\begin{array}{l}\text { Ask therapist for help when feeling } \\
\text { uncomfortable }\end{array}$ \\
\hline Ask patient to explain fear & Suggest or go to easier scenario \\
\hline Ask patient to explain scenario & Suggest or go to harder scenario \\
\hline $\begin{array}{l}\text { Help patient through the experience } \\
\text { through different techniques }\end{array}$ & Use other software options e.g. volume etc. \\
\hline Suggest patient to make progress & Quit \\
\hline
\end{tabular}

The main purpose of these controls is to make progress and gather the courage to progress to next level which is shown in table [4]. This feature can be implemented in VR through utilizing the level progression by use of SUDs. Voice assistance or other techniques can be used to encourage the user to deal with the phobia.

\subsection{Rewards and Scores}

Serious gaming with scores and rewards can motivate the user to make progress during VRET. Games are engaging and help user learn naturally. The feature of gamification should be added keeping the purpose of the therapy in mind. Gamifications should not create a barrier for the therapeutic effect of the system. The effectiveness of serious games in the treatment of mental illness was researched and proven [15]. VR makes it easier to develop serious games for phobia and anxiety by adding rewards and score to each level. The use of game engines available make easy translation of ideas possible.

There arises a question of increasing anxiety levels with anxiousness to make progress but a study has shown that casual games can be developed to reduce stress and improving mood [17]. Significant changes in anger, mood, confusion and fatigue were seen by playing the games designed for the study.

Thus gamification is a tool in the VRET development toolbox that should be utilized for making the experience as effective for patient as possible.

\subsection{Psychoeducation}

Anxiety and depression are most prevalent disorders thus there are many approaches to help patient receive the treatment that they are looking for. Psychoeducation intervention during CBT is one of the approaches. Psycho-education intervention over time can help in reduction of symptoms over time which proves it efficacy [18].

In VRET, these interventions can be provided to the patient as a separate package or as assistance during the therapy. Separate learning environment can be developed for the sake of educating the user. Psychoeducation is not limited to 
being aware of the illness or phobia but also includes the self-care solutions, information regarding stimuli and studies regarding the illness.

\section{CHALLENGES}

The self-help applications available in the commercial market are of questionable quality as they are not subjected to any evaluation. There is no criteria available to rate those application or their ability to help patients reduce their symptoms. No user tests or reviews are available to prove their efficacy. It is challenge to determine what works for self-help VRET and what doesn't. There are no tests made considering those applications that could totally assess the working of them. No subtle standards are being declared that could analyze the quality and efficiency of these applications and regarding the commercial market it cannot be determined that to what extent these applications work and there is no quantitative analysis to figure the percentage of reduction of the symptoms.

Even though with the advancement of technology and abundance of smart-phones, these applications can be made readily available but in the end, the question of whether they fulfill the purpose cannot be answered for each and every application. To develop state of the art next generation self-help VRET application test and trial method is not enough; all the design considerations few of which were listed in this paper should be kept in mind before development to get a perfect application that serves the objectives.

The availability of VR tools is still rare in developing countries like Pakistan, many people don't have access to it considering the economical conditions of the people and the high cost of the tools , According to a research carried out only $17.8 \%$ of the population uses internet, while addressing the factor that VR tools are rather very costly and are considered a thing only for the rich people it would be very hard to say if it would gain popularity among countries where the ratio of access to the internet is quite low because it is supposed to target those people who couldn't bear the cost going to a psychiatrist for their anxiety issues and consider it as a taboo doing that

A highly professional team is required for the designing and development of this sort of applications. Someone having a proper insight and first degree could come up with a perfect development strategy that meets all the requirement of the design and is beneficial in every way for the patients to treat their problems while remaining in a virtual environment. Anyone developing this type of application need to perfectly understand all the design constraints and then do a provision of the most perfect solution to it. The developer is needed to develop such an environment which is extremely user friendly because as long as the user is concerned he or she must be totally comfortable with the environment in order to outmaneuver the obstacles of the anxiety problems.

Coming up with a product that is in every aspect helpful to the user is not useful that addresses this type of scenario. A number of meaningful meetings are required between the psychiatrists and the developers so they could ensure the delivery of the product that surely reduces the symptoms of the issue lingering within the user .Developers and psychiatrists are needed to indulge themselves in the meaningful conversations to come up with the required results that serves all the objectives. 


\section{CONCLUSIONS}

Research has proven that VRET is as effective as CBT and the acceptance rate of VRET is higher in some cases than CBT. Use of these applications should not be limited therapist's office and should be made available to the public whenever it is possible.

These applications will be very beneficial in a long run if designed with keeping all the design constraints in mind. Although many design solutions are applicable but there is a subtle need of coming up with those scenarios that would help the users to overcome their anxiety challenges completely. They will be useful for the people who couldn't afford a therapist; they will be useful for the people who don't prefer going to a therapist and many other more benefits.

The main idea is to make them approachable to the laymen, and the provision of the scenarios that could serve as an all-time solution to the mental problems like phobias and anxiety without having one to put a diligent work on it. There is still a need to subject these applications to evaluation to make sure that they are fulfilling their purpose.

\section{REFERENCES}

[1] Krijn, M, et al. "Virtual Reality Exposure Therapy of Anxiety Disorders: A Review.” Clinical Psychology Review, vol. 24, no. 3, 2004, pp. 259-281., doi:10.1016/j.cpr.2004.04.001.

[2] Meyerbroker, Katharina. "Virtual Reality Exposure Therapy." The Wiley Handbook of Anxiety Disorders, Apr. 2014, pp. 1310-1324., doi:10.1002/9781118775349.ch63.

[3] Anand, Amit, Yu Li, Yang Wang, Jingwei Wu, Sujuan Gao, Lubna Bukhari, Vincent P. Mathews, Andrew Kalnin, and Mark J. Lowe. "Activity and connectivity of brain mood regulating circuit in depression: a functional magnetic resonance study." Biological psychiatry 57, no. 10 (2005): 1079-1088.

[4] Garcia-Palacios, A, et al. "Virtual Reality in the Treatment of Spider Phobia: a Controlled Study."Behaviour Research and Therapy, vol. 40, no. 9, 2002, pp. 983-993., doi: 10.1016/s0005-7967(01)00068-7.

[5] Banos, R.m., et al. "Virtual Reality Treatment of Flying Phobia." IEEE Transactions on Information Technology in Biomedicine, vol. 6, no. 3, 2002, pp. 206-212., doi:10.1109/titb.2002.802380.

[6] Garcia-Palacios, A., et al. "Comparing Acceptance and Refusal Rates of Virtual Reality Exposure vs. In Vivo Exposure by Patients with Specific Phobias." Cyber Psychology \& Behavior, vol. 10, no. 5, 2007, pp. 722-724., doi:10.1089/cpb.2007.9962.

[7] Lindner, Philip, Alexander Miloff, William Hamilton, Lena Reuterskiöld, Gerhard Andersson, Mark B. Powers, and Per Carlbring. "Creating state of the art, next-generation Virtual Reality exposure therapies for anxiety disorders using consumer hardware platforms: design considerations and future directions." Cognitive behaviour therapy 46, no. 5 (2017): 404-420.

[8] Lin, JJ-W., Henry Been-Lirn Duh, Donald E. Parker, Habib Abi-Rached, and Thomas A. Furness. "Effects of field of view on presence, enjoyment, memory, and simulator sickness in a virtual environment." In Virtual Reality, 2002. Proceedings. IEEE, pp. 164-171. IEEE, 2002.

[9] Brinkman, Willem-Paul, et al. "Therapist User Interface of a Virtual Reality Exposure Therapy System in the Treatment of Fear of Flying | Interacting 
with Computers | Oxford Academic.” OUP Academic, Oxford University Press, 27 Mar. 2010, academic.oup.com/iwc/article-abstract/22/4/299/935741/ The-therapist-user-interface-of-a-virtual-reality.

[10] Garner, Tom A. Echoes of Other Worlds: Sound in Virtual Reality, Past, Present and Future. Palgrave Macmillan, 2018.

[11] J. Schuemie, Martijn. (1999). Presence: Interacting in VR?

[12] Hoekstra, A. R. D. "3D audio for virtual reality exposure therapy." (2013).

[13] Lalwani, Mona. "For VR to Be Truly Immersive, It Needs Convincing Sound to Match.” Engadget, 14 July 2016, www.engadget.com/2016/01/22/vr-needs3d-audio/.

[14] Graham C.L. Davey (1991) Characteristics of individuals with fear of spiders, Anxiety Research, 4:4, 299-314, DOI: 10.1080/08917779208248798

[15] Lau, Ho Ming, et al. "Serious Games for Mental Health: Are They Accessible, Feasible, and Effective? A Systematic Review and Meta-Analysis." Frontiers in Psychiatry, vol. 7, 2017, doi:10.3389/fpsyt.2016.00209.

[16] Brinkman, Willem-Paul, Charles Van der Mast, Guntur Sandino, Lucy T. Gunawan, and Paul MG Emmelkamp. "The therapist user interface of a virtual reality exposure therapy system in the treatment of fear of flying." Interacting with computers 22, no. 4 (2010): 299-310.

[17] J., Parks. "A Randomized Controlled Study Measuring the Effectiveness of Casual Video Games in Reducing Stress and Increasing Mood." Frontiers in Neuroengineering, vol. 2, 2009, doi:10.3389/conf.neuro.14.2009.06.091.

[18] Houghton, Simon, and Dave Saxon. "An Evaluation of Large Group CBT Psycho-Education for Anxiety Disorders Delivered in Routine Practice." Patient Education and Counseling, vol. 68, no. 1, 2007, pp. 107-110., doi:10.1016/j.pec.2007.05.010. 


\section{AUTHORS}

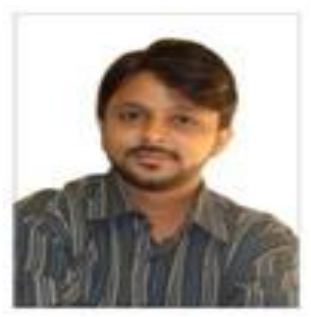

Dr. Muhammad Tahir received the BS degree in computer engineering from Sir Syed University, M.E. degree in Computer System from NED University and PhD in Information Science from University of Roma Tor Vergata. He is cur rently Associate Professor in Sir Syed University of Engineering and Technology, Karachi. His research interests include IP Switches/Routing, IPv4 Protocol, Firewall, IoT, Security Cryptography and Wireless Networks.

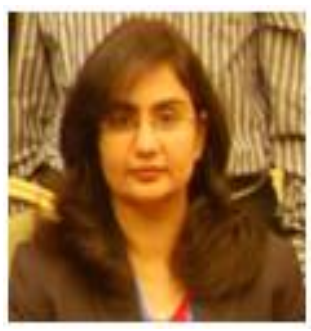

Dr. Rabia N. Enam received her PhD and Masters in Computer Engineering from Sir Syed University of Engineering and Technology (SSUET) Pakistan. She did Bachelors in Computer Engineering from N.E.D. University, Pakistan. Rabia also did Bachelors and Masters in Applied Mathematics from Karachi University. She is an Associate Professor at the Department of Computer Engineering at SSUET. Her research interests include the conceptual frameworks and algorithms used in Wireless Sensor Networks

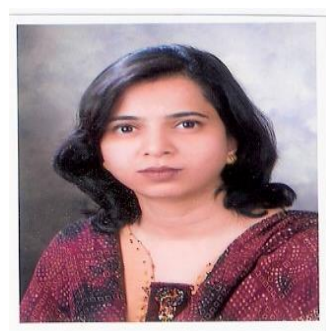

Najma Ismat is a $\mathrm{PhD}$ scholar at Sir Syed University of Engineering and Technology (SSUET) Pakistan. She has received a Masters and BS in Computer Engineering from Sir Syed University of Engineering and Technology (SSUET) Pakistan in 2002 and 1998 respectively. She is Assistant Professor in Department of Computer Engineering in SSUET. Her research interests are mobility and reliability in ad hoc wireless sensor networks 


\section{MACRO-E CONOMIC DETERMINANT AND INTERDEPE NDE NCE OF THE} STOCK MARKETS: EVIDENCE FROM EMERG ING ECONOMIES

Asim Rafiq

Department of Public Administration, Karachi University, Karachi, (Pakistan)

E-mail: asim_r83@hotmail.com

Shahbib Hassan

Department of Public Administration Karachi University, Karachi, (Pakistan)

E-mail: shassan@uok.edu.pk 


\section{ABSTRACT}

The purpose of the study is threefold. First, is to examine the long-term interdependence between China and the ten emerging economies, including Pakistan, Malaysia, Philippine, Indonesia, India, Hungary, Mexico, Russia, South Africa and Brazil using Johansen co-integration. Second, is to measure the timevarying interdependence between China and the other emerging economies using DDC GARCH model. Third, is to examine the impact of macroeconomic determinants on stock markets conditional correlations using panel regression. Monthly data from 2010 to 2016 is used. Results indicate that there is long-term interdependence between China and the other ten emerging economies. Furthermore, the results of DDC GARCH model support that China has a higher positive significant correlation with Pakistan, India, China, Indonesia, Malaysia, Philippine, Hungary, Mexico, Russia and South Africa. Finally, the results of the panel regression show that macroeconomic determinants have no significant effect on the equity market correlations between China and its companion emerging economies. It this, therefore, we can conclude that there is long run interdependence between the Chinese and the other emerging economies. Furthermore, this interdependence is also dynamic over the time. However, there is no significant impact of the macroeconomic determinants on the stock market interdependence between Chinese and the ten emerging economies.

\section{KEYWORDS}

Co-integration, DCC GARCH, Macro-economic determinants, Panel regression.

\section{INTRODUCTION}

Over the last few decades, government agencies and the key policymakers of both developing and the developed nations have attempted a few measures to abolish the hindrances among the nations to ensure the free stream of resources. This has significantly contributed to the interdependence of the economies and the effect of this association on their equity markets linkages. However, there are predominantly two distinct features in the realm of stock market interdependence. First, to what extent stock markets move together over the period. Second, what are the possible factors behind such a process? Earlier studies paid attention to the first aspect of the stock market interdependence for e.g.[1]-[4]. On the one hand, the more contemporary studies investigated the developed and the developing stock markets of USA, European, ASEAN and Asian markets. On the other hand, most of the previous studies examined the stock market interdependence in terms of correlation. Whereas, it is commonly believed that correlation has several deficiencies including the existence and the instability of lags. Therefore, even if the low correlation exists among the stock markets, this can be deceptive if it is timevarying [5]-[9].

Moreover, recently financial researchers have mainly devoted their attention to the [10]emerging economies stock markets for e.g. [6], [11], [12]. In view of that fact, emerging markets have distinctive characteristics from of the developed markets in terms of economic conditions, political structure, higher volatility, high interdependence, mean returns, currency, and the low correlation with the developed stock markets [13]-[15]. However, in these studies interdependence has been measured between emerging and the developed economies stock markets. 
On the one hand, some previous studies found a strong linkage between macroeconomic factors and the equity market, while some other found that these linkages are rather not robust [16], [17]. On the other hand, [18] investigated what are the factors behind the stock market interdependence of emerging economies. [19]-[21] studies the cross-market linkages between Australia and its trading partners and found trade ties result in equity market interdependence.

But to our best knowledge, there is no major contribution regarding stock market interdependence in emerging economies after the Pretorius because financial crises divert the attention of the researchers. So, in this study it is endeavor first, to fill up the gap and identify what macroeconomic factors are behind the stock market interdependence in emerging economies context, as emerging economies grow in number since last twenty years and secondly, there is dearth of empirical investigation on stock market interdependence that takes the viewpoint of emerging economies, the use of emerging economies perspective provides an opportunity to address this particular gap.

This paper is also different from the other papers in the sense that in this paper the major driver of equity market belongs inside the emerging economy which is Chinese stock market rather than any developed world like in other studies for e.g. US equity markets are considered to be the benchmark equity market as the significance of China is the second largest and shares the major world output among the top ten economies. Moreover, presently China share has grown to $15.1 \%$, while the share of Japan and the USA has fallen down to $31.1 \% \%$ by 2017 [22].

The aim of this paper is threefold. First, is to observe the interdependence among the emerging economies stock markets, second, measure the time varying relationship among the stock markets of emerging economies. Finally, is to detect the possible macroeconomic determinants behind the interdependence among these markets. The subject of stock market interdependence has immense, theoretical, policy and practical significance. The foremost benefit of the interdependent market is that cost of and access to foreign investment lower and easier, respectively. To achieve the objective of the study first, we employ the Johansen multivariate co-integration test to ascertain long term association (interdependence). Second, to examine the time-varying association we employ DCG GARCH model. Finally, to detect the influence of macroeconomic variables on the security market interdependence this study employs a panel regression model.

\section{LITERATURE REVIEW}

Stock market interdependence has been tested employing several techniques but the empirical shreds of evidence are mixed. Studies focus on the co-integration techniques includes: [23] was among the first to implement the co-integration technique for the analysis of the interdependence among the United Kingdom and Japan, Germany and USA after the abolition of the currency restrictions in the United Kingdom. It was found that UK equity market was correlated with all except the USA market. 
Researchers commonly belief that global diversification benefits can only be reaped if the correlation between the equity markets is low. However, the correlation between the equity markets is not stable over the time. It is, therefore, crucial to understand the instability of the correlation over the time. Initially, [24] researched the instability of the correlation and the covariance and found that correlation is stable over a fifteen year period during 1967-82. The results from past studies are mixed because most of the previous literature examined the market interdependence under the liner frame.

However, the liner co-integration technique unable to present whether the equity markets have become more integrated or whether the process of integration is gradual. According to [13] market interdependence is time-varying. To address this issue several studies consider the non-linear framework. [8] Uses the complex network analysis and the corresponding correlation measure to examine the underlying dynamic interdependence of the equity markets. [6] examined the twenty-two emerging equity markets situated in Europe, Asia, America, and the Africa/Middle East with the US market by applied a different approach as most of the researchers used, which is wavelet theory for empirical testing and It was found that the integrated intensity of the stock markets is time varying. [25] Tested the dynamic conditional correlation between the Chines and the international stock markets. It is substantiated through the results that correlations across the markets are time-varying. It is also identified that dynamic correlation is compactly linked with the geographic location. [26] Also confirmed that dynamic conditional correlation between S\&P 500 and S\&PGSCI energy sub-index is time- varying. It is therefore, we can infer that interdependence between the equity markets is dynamic and it is a gradual process.

So based on the literature the DDC GARCH methodological approach has succeeded in capturing is the dynamic conditional correlation. It permits the researchers to comprehend the change in the conditional correlation and the volatilities which is the more precise representation of the fact.

Literature gives an exposition of the theoretical understanding of why the comovement between the stock markets exist. on the one hand, variables those are perceived to be the main driver of the interdependence of the stock market in the developed economies are bilateral trade, exchange rate volatility, Size differential, market volatility, size differential, real interest rate differential, term structure differential, industrial composition and return on world market index.

Alternatively, the variables that influence the stock market interdependence are still mainly undiscovered in case of emerging equity markets. In general changes in these variables over the time also affects the stock market interdependence. According to the early studies, foreign trade promotes business cycle harmonization through the countries and consequently impacts the degree of their market interdependence for example [27]-[30] ascertained that trade is a significant variable in describing the correlation between the stock markets. similarly, Pretorius (2002) if interdependence between two economies due to bilateral trade, consequently, one can anticipate that there stock market and the economy will move 
in the similar route. Based on these theoretical and empirical pieces of evidence it is concluded that trade is a significant factor in describing the interdependence.

Numerous past studies use exchange rates volatility and the inflation differential to observe their impact on stock market interdependence [18]. The outcomes from these limited studies indicate that the exchange rate has a significant effect on the equity market interdependence [31]-[33]. Similarly, [34] also confirm the previous findings and documented negative effect of exchange rates on stock market interdependence.

Based on the above critical review of the literature it can be argued that market interdependence is a conflicting issue. This implies that it is a time-varying phenomenon even among the similar markets and furthermore, it also varies among countries development level for e.g. developed, developing, underdeveloped and emerging.in addition to this, bilateral trade relationships, industrial production, inflation, exchange rate are possible factors in describing the stock market interdependence.

In the process of the review of the literature, we find the gap in three areas first, there is a number of studies conducted in the most mature and the developed world and mostly the benchmark economy have been US stock market. Secondly, most of the studies use correlation or the co-integration to measure the stock market interdependence. Lastly, there is no comprehensive study after the [18] which reexamine the interdependence of the stock markets in terms of economic interdependence among the emerging economies stock market using the DDC GARCH model to also consider time-varying behavior.

\section{DATA AND METHODOLOGY}

On the one hand, we Johansen co-integration to examine long run static interdependence. On the other hand we applied DCC GARCH model to assess the long run time-varying interdependence. Following are the representative index of each country. KSE 100 index (Pakistan), S\&P BSE SENSEX (India), SSE Composite (China), JSKE (Indonesia), FTSE Bursa Malaysia KLCI (Malaysia), PSEi (Philippine), BVSP (Brazil), BUX (Hungary), MMX (Mexico), MICEX (Russia) and FTSE/JSE (South Africa).

Further, to study the impact of macroeconomic determinant on stock market interdependence we run a Panel regression model. For this purpose we collected data of differential of bilateral trade, inflation (CPI), interest rate and exchange rates (local currency) from IMF Financial statistics database. Only bilateral trade data is transformed into natural logarithm form and the other factors are used in their initial form.

In this study, we apply the well- known multivariate GARCH model, namely the DDC GARCH model. The key benefit of the DDC GARCH model as compared to other time-varying models, for instance, Flexible Least Square and Kalman filters are that it permits the researcher to understand the shifts in conditional correlations and volatilities which is the more correct picture of the reality. Lastly, the panel regression techniques is an effective and efficient in terms of measuring the cause 
and effect of industrial production, bilateral trade, exchange rate, inflation and interest rate on time-varying conditional correlations of stock market returns.

\section{EMPIRICAL RESULTS}

\subsection{Multivariate co-integration test}

After unit root testing as a prerequisite condition for co-integration testing, we examine the long-term co-movement between China and the other emerging economies by employing the multivariate VAR based co-integration technique developed by [35], [36]. Results of the multivariate co-integration are discussed below.

Table 1. Multivariate co-integration.

\begin{tabular}{|c|c|c|c|c|}
\hline \multicolumn{4}{|c|}{ Unrestricted Cointegration Rank Test (Trace) } & \\
\hline Hypothesized & & Trace & 0.05 & \\
\hline No. of CE(s) & Eigenvalue & Statistic & Critical Value & Prob.** \\
\hline None * & 0.620 & 406.469 & 285.143 & 0.000 \\
\hline At most $1 *$ & 0.543 & 323.234 & 239.235 & 0.000 \\
\hline At most $2 *$ & 0.516 & 255.852 & 197.371 & 0.000 \\
\hline At most $3 *$ & 0.468 & 193.523 & 159.530 & 0.000 \\
\hline At most $4 *$ & 0.435 & 139.285 & 125.615 & 0.005 \\
\hline At most 5 & 0.270 & 90.238 & 95.754 & 0.113 \\
\hline At most 6 & 0.245 & 63.132 & 69.819 & 0.152 \\
\hline At most 7 & 0.198 & 38.960 & 47.856 & 0.262 \\
\hline At most 8 & 0.123 & 19.959 & 29.797 & 0.426 \\
\hline At most 9 & 0.089 & 8.6380 & 15.494 & 0.310 \\
\hline At most 10 & 0.007 & 0.609 & 3.841 & 0.435 \\
\hline \multicolumn{5}{|c|}{ Trace test indicates 5 co-integrating eqn(s) at the 0.05 level } \\
\hline \multicolumn{5}{|c|}{ Unrestricted Cointegration Rank Test (Maximum Eigenvalue) } \\
\hline Hypothesized & & Max-Eigen & 0.05 & \\
\hline No. of CE(s) & Eigenvalue & Statistic & Gritical Value & Prob.** \\
\hline None * & 0.620 & 83.235 & 70.535 & 0.002 \\
\hline At most $1 *$ & 0.543 & 67.382 & 64.505 & 0.026 \\
\hline At most $2 *$ & 0.516 & 62.329 & 58.434 & 0.020 \\
\hline At most $3 *$ & 0.468 & 54.238 & 52.363 & 0.032 \\
\hline At most $4 *$ & 0.435 & 49.047 & 46.231 & 0.024 \\
\hline At most 5 & 0.270 & 27.106 & 40.078 & 0.626 \\
\hline At most 6 & 0.245 & 24.171 & 33.877 & 0.443 \\
\hline At most 7 & 0.198 & 19.001 & 27.584 & 0.415 \\
\hline At most 8 & 0.123 & 11.321 & 21.132 & 0.615 \\
\hline At most 9 & 0.089 & 8.029 & 14.265 & 0.376 \\
\hline At most 10 & 0.007 & 0.609 & 3.841 & 0.435 \\
\hline \multicolumn{5}{|c|}{ Max-eigenvalue test indicates 5 cointegrating eqn(s) at the 0.05 level } \\
\hline \multicolumn{5}{|c|}{ * denotes rejection of the hypothesis at the 0.05 level } \\
\hline
\end{tabular}

Grounded on these outcomes, the null hypothesis of no co-integration between these markets can be rejected (see. Table. 2). These outcomes are similar to [4], [21], [37], [38]. It is, therefore, on the basis of results we reject the null hypothesis as stated below:

Ho: There is no long-run relationship between emerging and Chinese stock markets. 
Table 2. Time-varying conditional correlations Between China and each country.

\begin{tabular}{|l|l|c|c|}
\hline \multicolumn{1}{|c|}{ Country } & \multicolumn{1}{|c|}{ Index } & $\begin{array}{c}\text { Correlation } \\
\text { coefficient }\end{array}$ & P-value \\
\hline Pakistan & KSE & 0.845 & 0.000 \\
\hline Malaysia & KLCI & 0.813 & 0.000 \\
\hline Philippine & PSEi & 0.834 & 0.000 \\
\hline Indonesia & IKSE & 0.834 & 0.000 \\
\hline India & BSENSEX & 0.860 & 0.000 \\
\hline Hungary & BUX & 0.947 & 0.000 \\
\hline Mexico & MMX & 0.915 & 0.000 \\
\hline Russia & MICEX & 0.957 & 0.000 \\
\hline South Africa & ISE & 0.986 & 0.000 \\
\hline Brazil & BVSP & -0.708 & 0.000 \\
\hline
\end{tabular}

Table 2 testifies the conditional correlations between China and the rest of emerging economies in MSCI index. The calculated correlations in the above table support that China has the higher positive significant correlation with Pakistan, India, China, Indonesia, Malaysia, Philippine, Hungary, Mexico, Russia and South Africa. However, Brazil is an exception with higher negative significant correlation with China stock market. On the basis of the empirical analysis of the time-varying behavior of stock markets, we can reject the below mentioned null hypothesis.

$\mathrm{H}_{\mathrm{o}}$ : There is no dynamic relationship between china and the other emerging economies.

\subsection{Impact of macro-economic determinants on stock market correlations using Panel regression model}

To analyze the influence of the macroeconomic determinants on the stock market correlations between the China and the emerging economies we apply the panel regression model.

Table 3. Result of Random Effect Model.

Dependent Variable: Correlation

Method: Panel EGLS (Cross-section random effects)

\begin{tabular}{|c|c|c|c|c|}
\hline Variable & Coefficient & Std. Error & t-Statistic & Prob. \\
\hline C & 4.633 & 8.500 & 0.545 & 0.585 \\
\hline D(Trade) & 8.899 & 5.788 & 0.015 & 0.987 \\
\hline D(Exchange rate) & -9.811 & 9.988 & -0.098 & 0.922 \\
\hline D(Industrial Production) & 2.499 & 1.277 & 0.196 & 0.845 \\
\hline D(INFLATION) & -2.688 & 2.699 & -0.999 & 0.318 \\
\hline D(Interest rate) & -0.001 & 0.000 & -1.753 & 0.080 \\
\hline
\end{tabular}

The result of random effect model presents that there is no single macroeconomic determinant among bilateral trade, inflation differential, interest rate differential, industrial production differential and exchange rate differential, which report the significance. The results show that macroeconomic determinants have no significant effect on the stock market correlations between China and its companion emerging economies. It is, therefore, we cannot reject the null hypothesis as stated below: 
Ho: There is no significant impact of macroeconomic determinants on stock market correlations between China and emerging economies.

It is therefore, we can conclude macroeconomic determinants have insignificant impact on the stock market correlations between China and the emerging economies stock markets. However, the results of this study are dissimilar to those of [39], [40] who document that macroeconomic linkages among the countries can drive their stock market interdependence.

\section{CONCLUSION}

In this paper, first, we investigate the interdependence from the perspective of China and its companion emerging economies stock markets including Chinese, Pakistan, Malaysia, Indonesia, Philippine, Brazil, Mexico, Hungary, Russia, South Africa, and India, through the application of multivariate Johansen co-integration technique. Second, we determine the degree of the interdependence between these markets, through the application of DDC GARCH model. Third, we examine what macroeconomic determinants are significant in establishing the interdependence between China and its companion emerging economies, this study has empirically analyzed the dynamic association between the equity markets and the macroeconomic determinants using panel regression analysis.

Results indicate that Chinese stock market are co-integrated with stock market of the other emerging markets. Centered on these outcomes, the null hypothesis of no co-integration between these markets can be rejected. These outcomes are consistent with the earlier studies like (Masih and Masih, 1999; Shamsuddin and Kim, 2003; Kazi, 2008; Paramati, Gupta and Roca, 2015). In addition to this, The results of DDC GARCH model support that China has a higher positive significant correlation with Pakistan, India, China, Indonesia, Malaysia, Philippine, Hungary, Mexico, Russia and South Africa. However, Brazil is an exception with higher negative significant correlation with the Chinese stock market.

It also confirms that the relationship between China and the other emerging economies has been increasing over the time except for Brazil. Finally, the results of the panel regression show that macroeconomic determinants have no significant effect on the equity market correlations between China and its companion emerging economies. It this, therefore, we can conclude that there is long run interdependence between the Chinese and the other emerging economies.

Furthermore, this interdependence is also dynamic over the time. However, there is no significant impact of the macroeconomic determinants on the stock market interdependence between Chinese and the other emerging economies. The outcomes of this study will significantly contribute to the current literature, from the perspective of both the investors and the policymakers.

\section{ACKNOWLEDGEMENTS}

We thanks all who support in this paper. 


\section{REFERENCES}

[1] F. M. Longin and B. Solnik, "Is the correlation in international equity returns constant," J. Int. Money Financ., vol. 14, no. 1, pp. 3-26, 1995.

[2] T. Choudhry, "Interdependence of stock markets : evidence from Europe during the 1920s and 1930s Interdependence of stock markets : e v idence from Europe during the 1920s and 1930s," Appl. Financ. Econ., vol. 6, no. November 2014, pp. 243-249, 1996.

[3] G. A. karolyi and R. Stulz, "Why Do Markets Move Together? An Investigation of U.S.-Japan Stock Return Comovements," J. Finance, vol. 51, no. 3, pp. 951986, 1996.

[4] A. M. M. Masih and R. Masih, "Are Asian stock market fluctuations due mainly to intra-regional contagion effects? Evidence based on Asian emerging stock markets," Pacific-Basin Financ. J., vol. 7, pp. 251-282, 1999.

[5] M. E. Arouri, H. Ben Ameur, N. Jawadi, F. Jawadi, and W. Louhichi, "Are Islamic finance innovations enough for investors to escape from a financial downturn ? Further evidence from portfolio simulations," Appl. Econ., vol. 45, no. May 2013, pp. 37-41, 2012.

[6] M. Graham, J. Kiviaho, and J. Nikkinen, "Integration of 22 emerging stock markets: A three-dimensional analysis," Glob. Financ. J., vol. 23, no. 1, pp. 3447, 2012.

[7] G.-J. Wang, C. Xie, M. Lin, and H. E. Stanley, "Stock market contagion during the global financial crisis: A multiscale approach," Financ. Res. Lett., vol. 0, pp. $1-6,2017$.

[8] X. Zhang, X. Zheng, and D. D. Zeng, "The dynamic interdependence of international financial markets: An empirical study on twenty-seven stock markets," Physica A, vol. 472, pp. 32-42, 2017.

[9] A. N. Ajmi, S. Hammoudeh, D. K. Nguyen, and S. Sarafrazi, "How strong are the causal relationships between Islamic stock markets and conventional financial systems? Evidence from linear and nonlinear tests," J. Int. Financ. Mark. Institutions Money, vol. 28, no. 1, pp. 213-227, 2014.

[10] M. Ranta, "Contagion among major world markets: a wavelet approach," Int. J. Manag. Financ., vol. 2, no. 9, pp. 133-149, 2013.

[11] J. Fidrmuc and I. Korhonen, "Journal of Asian Economics," J. Asian Econ., vol. 21, no. 3, pp. 293-303, 2010.

[12] C. Stijn, G. Deniz, Dell'Ariccia, Igan, and L. Luc, "Cross-country experiences and policy implications from the global financial crisis," Econ. Policy, vol. 25, no. 62, pp. 267-293, 2010.

[13] G. Bekaert and C. R. Harvey, "Time-Varying World Market Integration," vol. L, no. 2, 1995.

[14] C. B. Erb, C. R. Harvey, and T. E. Viskanta, "Forecasting International Equity Correlations," Financ. Anal. J., vol. 50, no. 6, pp. 32-45, 1994.

[15] V. Ragunathan, R. W. Faff, and R. D. Brooks, "Correlations, business cycles and integration," J. Int. Financ. Mark. Institutions Money, vol. 9, pp. 75-95, 1999.

[16] R. Verma and T. Ozuna, "Are emerging equity markets responsive to crosscountry macroeconomic movements? Evidence from Latin America," vol. 15, pp. 73-87, 2005.

[17] R. Kizys and C. Pierdzioch, "Changes in the international comovement of stock returns and asymmetric macroeconomic shocks," J. Int. Financ. Mark. Institutions Money, vol. 19, pp. 289-305, 2009. 
[18] E. Pretorius, "Economic determinants of emerging stock market interdependence," Emerg. Mark. Rev., vol. 3, no. 1, pp. 84-105, 2002.

[19] S. R. Paramati, E. Roca, and R. Gupta, "Economic integration and stock market dynamic linkages : evidence in the context of Australia and Asia," Appl. Financ. Econ., vol. 48, no. 44, pp. 4210-4226, 2016.

[20] S. R. Paramati, A. Zakari, M. Jalle, and P. Begari, "The dynamic impact of bilateral trade linkages on stock market correlations of Australia and China," Appl. Econ. Lett., vol. 00, no. 00, pp. 1-5, 2017.

[21] S. R. Paramati, R. Gupta, and E. Roca, "Stock market interdependence between Australia and its trading partners : does trade intensity matter ? Stock market interdependence between Australia and its trading partners : does trade intensity," no. June, pp. 37-41, 2015.

[22] "IMF Economic Outlook," 2017. [Online]. Available: https://www.imf.org/en/Publications/WEO/Issues/2017/09/19/worldeconomic-outlook-october-2017.

[23] M. P. Taylor, I. Tonks, S. The, N. May, M. P. Taylor, and I. Tonks, "The Internationalization of Stock Markets and the Abolition of UK exchange control," reveiw Econ. Stat., vol. 71, no. 2, pp. 332-336, 1989.

[24] B. Dumas, C. Harvey, E. Kaplanis, and K. Kroner, "Is the correlation in international equity returns constant: 1960-1990?," J. i nternational money Financ., vol. 7, no. 1, pp. 63-75, 1988.

[25] T. C. Chiang and X. Chen, "Empirical Analysis of Dynamic Linkages between China and International Stock Markets," J. Math. Financ., vol. 06, no. 01, pp. 189-212, 2016.

[26] B. Buyuksahin and M. Robe, "Does' paper oil'matter? Energy markets' financialization and equity-commodity co-movements.," 2011.

[27] H. Asgharian, W. Hess, and L. Liu, "A spatial analysis of international stock market linkages," J. Bank. Financ., vol. 37, no. 12, pp. 4738-4754, 2013.

[28] M. Beine and B. Candelon, "Integration and stock market co-movement between emerging economies," Quant. Financ., vol. 11, no. 2, pp. 299-312, 2006.

[29] F. Chen, N. F., \& Zhang, "Correlations, trades and stock returns of the PacificBasin markets," Pacific-Basin Financ. J., vol. 5, no. 5, pp. 559-577, 1997.

[30] S. Walti, "The macroeconomic determinants of stock market synchronization," J. Int. Bank. Law, vol. 11, no. 10, pp. 1-30, 2005.

[31] K. Bracker, D. Scott, and P. D. Koch, "Economic determinants of evolution in international stock market integration," J. Empir. Financ., vol. 6, pp. 1-27, 1999.

[32] K. J. Forbes and R. Rigobon, "No Contagion, Only Interdependence: Measuring Stock Market Comovements," J. Finance, vol. 57, no. 5, pp. 22232261, 2002.

[33] C. Lin and W. Cheng, "Economic determinants of comovement across international stock markets: the example of Taiwan and its key trading partners," Appl. Econ., vol. 40, no. December 2013, pp. 37-41, 2008.

[34] J. Tavares, "Economic integration and the comovement of stock returns," Econ. Lett., vol. 103, no. 2, pp. 65-67, 2009.

[35] S. Johansen, "Johansen, Søren. Estimation and hypothesis testing of cointegration vectors in Gaussian vector autoregressive models.," Econom. J. Econom. Soc., pp. 1551-1580, 1991. 
[36] S. Johansen, "Identifying restrictions of linear equations with applications to simultaneous equations and cointegration," J. Econom., vol. 69, no. 1, pp. 111$132,1995$.

[37] M. H. Kazi, "Is Australian stock market integrated to the equity markets of its major trading partners ?," Int. Rev. Bus. Res. Pap., vol. 4, no. 5, pp. 247-257, 2008.

[38] A. F. M. Shamsuddin and J. H. Kim, "Integration and interdependence of stock and foreign exchange markets: an Australian perspecti v e," J. Int. Financ. Mark. Institutions Money, vol. 13, pp. 237-254, 2003.

[39] M. Abdul Karim, B., \& Shabri Abd. Majid, "Does trade matter for stock market integration?,” Stud. Econ. Financ., vol. 27, no. 1, pp. 47-66, 2010.

[40] G. Fazio, "Extreme interdependence and extreme contagion between emerging markets," J. Int. Money Financ., vol. 26, no. 8, pp. 1261-1291, 2007. 
Macro-Economic determinant and interdependence of the stock markets: evidence from emerging economies DOI: http://dx.doi.org/10.17993/3ctecno.2019.specialissue.03 


\title{
ON CONVERGE NCE OF ITERATIVE METHOD FOR DETERMINATION OF WEIBULL PARAMETERS BY MAXIMUM LIKE LIHOOD METHOD
}

Fida Hussain Khoso

Dawood University of Engineering \& Technology, Karachi, (Pakistan) E-mail: fida.dcet74@gmail.com

Dr. Gasim Alandjan

Yanbu University College, Yanbu, (Saudi Arabia)

E-mail: alandjanig@rcyci.edu.sa

\begin{abstract}
Altaf Hussain Bouk
Yanbu University College, Yanbu, (Saudi Arabia)

E-mail: bouka@rcyci.edu.sa
\end{abstract}

Prof. Dr. Engr. Sayed Hyder Abbas Musavi

Indus University, Karach, (Pakistan)

E-mail: dean@indus.edu.pk 


\section{ABSTRACT}

The Weibull distribution is frequently used for the assessment of wind energy potential and modeling of wind speed data. The parameters of Weibull distribution are determined by a number of methods; Maximum Likelihood Methods is one of them. The values of scale and shape parameters of Weibull distribution are found by the help of Maximum Likelihood function. Two different techniques are used to find the parameters. One is known as iterative method, in which a start value of ' $k$ ' is set and iterations are terminated when given criterion is reached. The second method is Newton Raphson method of finding roots. We report here a problem of non-convergence of iterative method. We suggest the Newton Raphson method as the best choice for finding the value of ' $k$ ' through Maximum Likelihood Method.

\section{KEYWORDS}

Weibull distribution, Weibull parameter, Maximum Likelihood Method.

\section{INTRODUCTION}

We are living in machine era; people, at work place have been replaced by machines or robots. At home too, daily routine works are done by electronic devices. The use of electricity, have increased drastically in last four decades. Fast depletion of fossil fuels has made people around the world to think for alternate source of energy. Uninterrupted, cost effective, and environmental friendly source of energy is a dream and desire of today's world. Wind energy is a good choice as an alternate source of energy. Many parts of world have got excellent potential of wind speed; wind energy is rapidly growing as a source of energy around the world [1,2]. Most of the countries have been generating electrical energy through wind [3].

Wind fluctuates time to time, the fluctuations also depends on the height from the sea level. Large amplitude fluctuations are the challenges in designing and installing wind farms [4-6]. The planning, designing, installing and operating wind turbines depends on wind potential and its characteristics [7].

The modeling of wind power plays in important role in assessing wind potentials [8]; different statistical distributions and mathematical techniques have been employed to model wind data [9]. Most widely used statistical distribution to model wind data is Weibull distribution [10]. There are different forms of Weibull distribution depending upon no. of parameters. The simplest Weibull distribution has two parameters; its Probability Density Function (PDF) is given in eq. (1)

$f(v)=\frac{k}{c}\left(\frac{v}{c}\right)^{k-1} \mathrm{e}^{-\left(\frac{v}{c}\right)^{k}}$

Here ' $\mathrm{k}$ ' is known as shape parameter and 'c' is known as scale parameter. The cumulative distribution function (CDF) is given by eq. (2):

$$
F(v)=1-\mathrm{e}^{-\left(\frac{v}{c}\right)^{k}}
$$

\section{ANALYSIS}

Various statistical and mathematical methods are employed to find parameters ' $\mathrm{k}$ ' and 'c'. Among them are Methods of moment, Empirical Method, Energy Pattern Factor Method, Graphic Method, Least Square Method, Equivalent Energy 
Method, Maximum Likelihood Method, and Modified Maximum Likelihood Method. In this study we considered only Maximum Likelihood Method. The values of Weibull parameters by this method are given by equations (3) and (4); 'k' and 'c' are found by iterative method or by Newton Raphson Method.

\subsection{Iterative Method}

In iterative method a start value of ' $\mathrm{k}$ ' is selected and wind speed data is used to calculate sums in eq. (3), since sum of Logarithm of wind speeds is needed in the calculation, hence zero wind speeds are neglected in this method. The new value of ' $k$ ' is generated through eq. (3) and used in next iteration, the process continues until a given criterion is reached.

$$
\begin{aligned}
& k=\left[\frac{\sum_{i}^{n} v_{i}^{k} \ln \left(v_{i}\right)}{\sum_{i}^{n} v_{i}^{k}}-\frac{\sum_{i}^{n} \ln \left(v_{i}\right)}{n}\right]^{-1} \\
& c=\frac{\sum_{i}^{n} v_{i}^{k}}{n}
\end{aligned}
$$

\subsection{Newton Raphson Method}

The eq. (5) obtained by differentiating Logarithm of Likelihood function with respect to shape parameter ' $k$ ' is used as a function of ' $k$ ' in Newton Raphson Method of finding roots.

$$
f(k)=\frac{n}{k}-n \ln c+\sum_{i=1}^{n} \ln \left(v_{i}\right)-\sum_{i=1}^{n}\left(\frac{v_{i}}{c}\right)^{k} \ln \left(\frac{v_{i}}{c}\right)=0
$$

An initial value of ' $k$ ' is selected as a starting point of Newton's method. Wind data of two coastal regions of Pakistan, namely, Gwadar and Ormara are used to calculate Weibull parameters by Maximum Likelihood Method. To investigate any dependence of calculation method (iterative and Newton's method) on start value of ' $k$ '; various start values of ' $k$ ' are employed to calculate parameters. A dependence on start value of ' $k$ ' is found and shown in figures (1-3).

The iterative method is easy to implement but convergence is not guaranteed. Table 1 gives the results of iterative method with various start values of ' $k$ '. The iteration oscillates between two values and does not converge. 


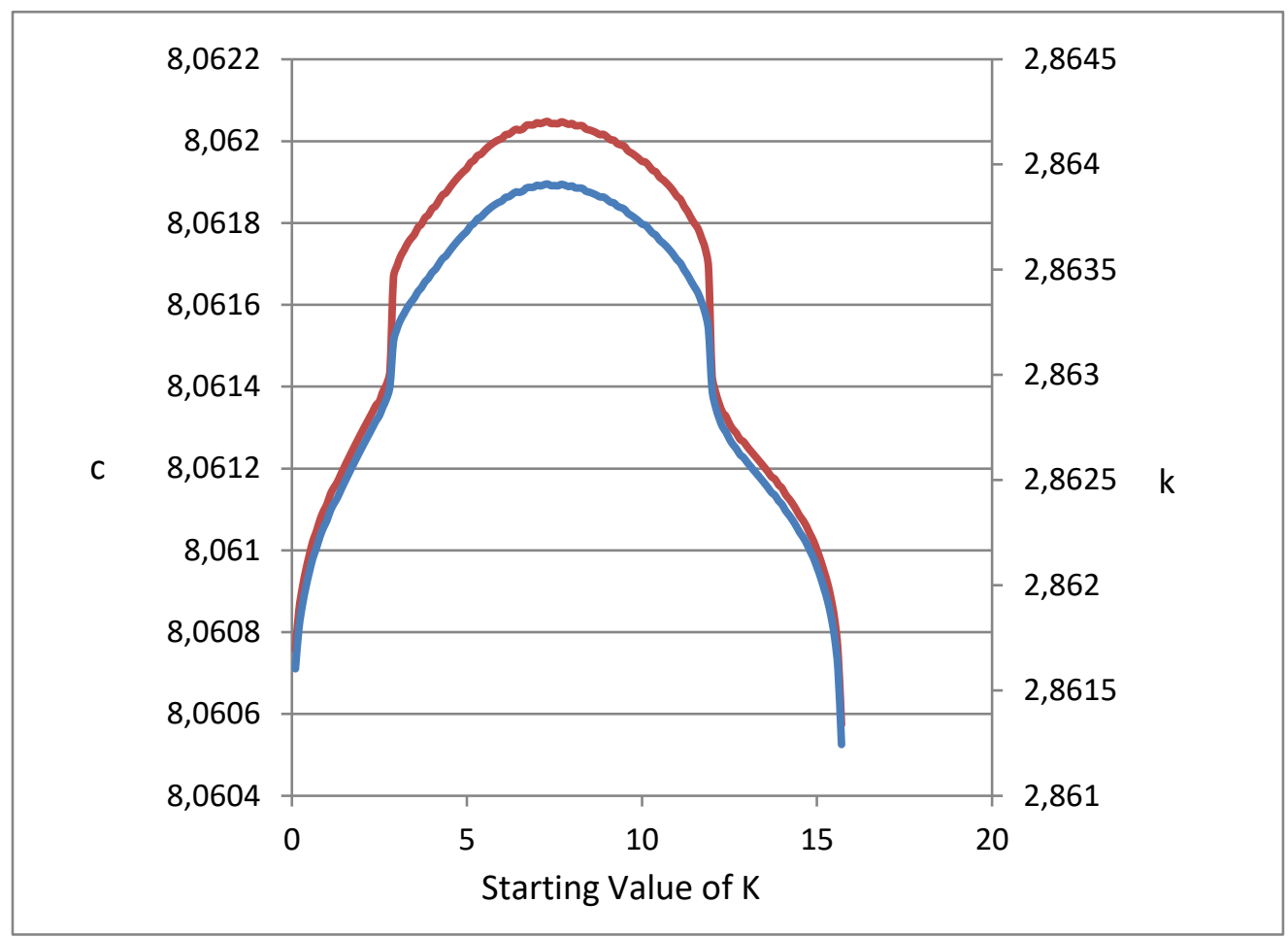

Figure 1. Variation of $k$ and c with various start values of 'k' for Gwadar's wind data of Jan 2002.

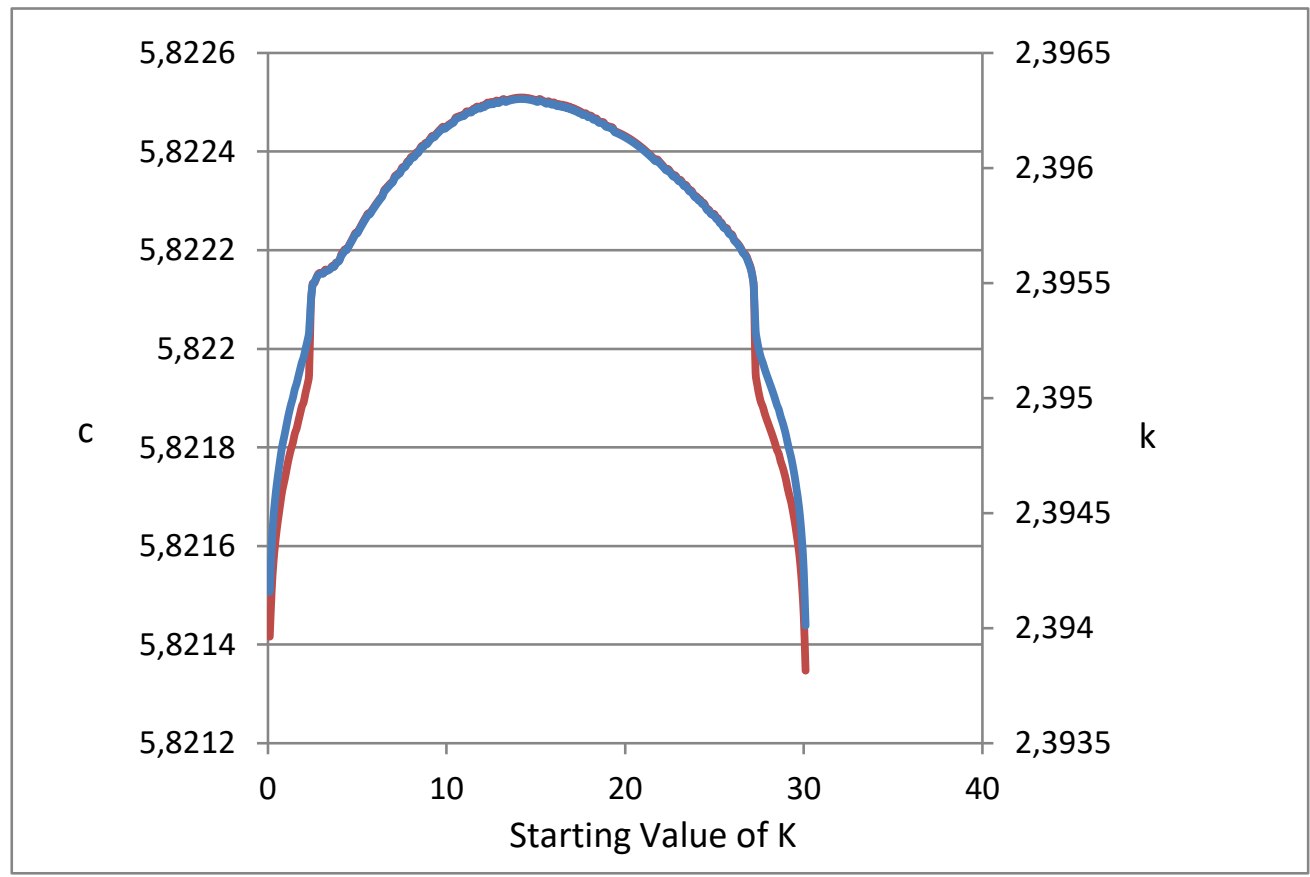

Figure 2. Variation of $k$ and $c$ with various start values of ' $k$ ' for Ormara's wind data of May. 


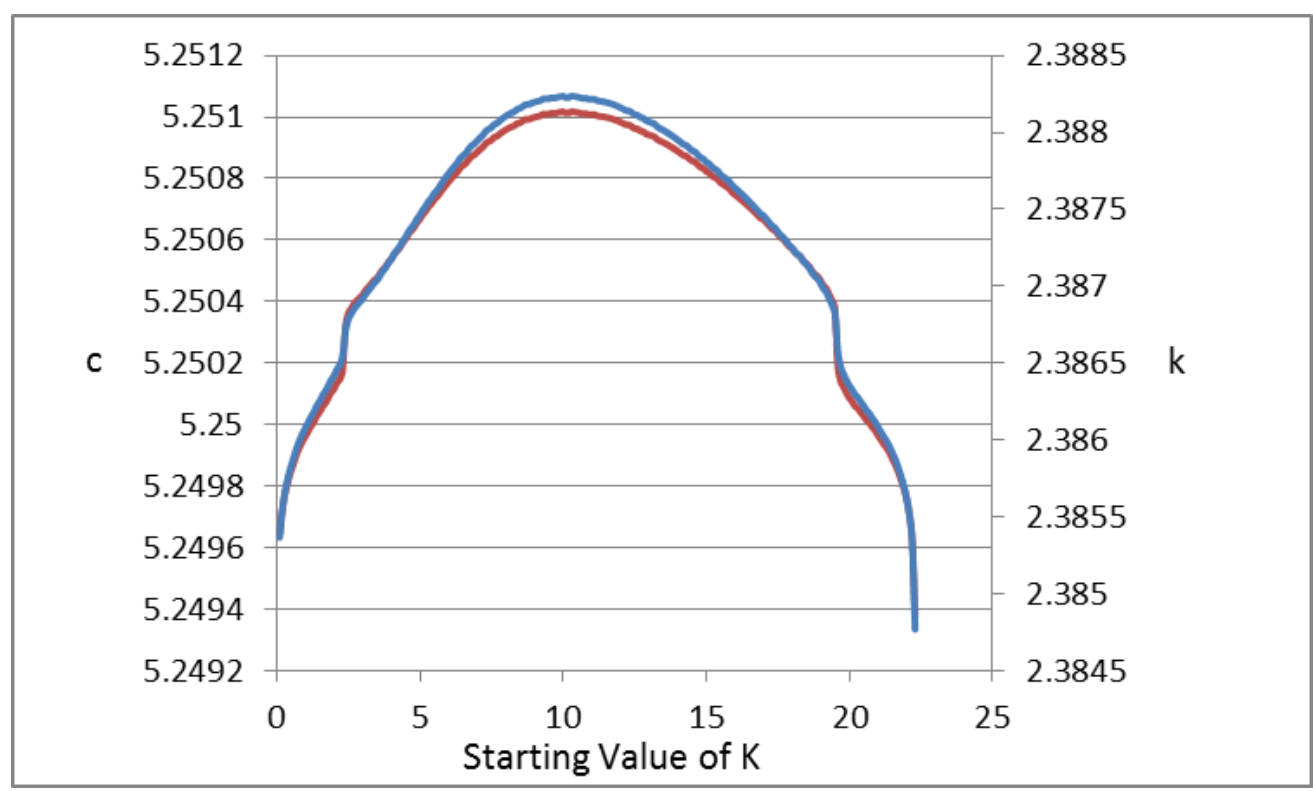

Figure 3. Variation of $k$ and $c$ with various start values of ' $k$ ' for Ormara's wind data of July.

\section{CONCLUSION}

Wind data of Gwadar (Jan 2002) and ten years data of Ormara have been used in this study. In figs (1-3) the results of Newton Raphson method are plotted. Various start values (starting from 0.1 and step size of 0.1) are taken to find Weibull distribution parameters. It is found that the values of scale and shape parameters increase with increasing start value of ' $k$ '. Both approach to a maximum value at some start value of ' $k$ '. If the start value further increased the shape and scale parameters start decreasing. The variation in shape parameter is less than 0.003 and in scale parameter it is less than $0.005 \mathrm{~m} / \mathrm{s}$.

Iterative method for finding Weibull parameters through Maximum Likelihood method was used for Gwadar. Various start values of ' $\mathrm{k}$ ' were taken to find the parameters. It was found that the iterative method does not converge; the shape parameter ' $k$ ' oscillates between two values. One value generates the other, and convergence criterion does not approach. Table I shows the results of iterative method for start values of $\mathrm{k}=1,2,3,4,5,10,20,50$. It can be seen that the iterations oscillate between two values 1.559878 and 5.466348 . Hence, it is likely that the iterative method would not converge. It is suggested that if Weibull parameters are determined by Maximum Likelihood Method, preference to Newton Raphson method should be given over to Iterative method discussed above. 
Table 1. The results of iterative method for start value of $k=1,2,3,4,5,10,20$, and 50 .

\begin{tabular}{|c|c|c|c|c|c|c|c|c|}
\hline & \multicolumn{2}{|c|}{$k=1$} & \multicolumn{2}{|c|}{$k=2$} & \multicolumn{2}{|c|}{$k=3$} & \multicolumn{2}{|c|}{$k=4$} \\
\hline $\begin{array}{c}\text { Iteration } \\
\#\end{array}$ & K(i) & $K(i+1)$ & $K(i)$ & $K(i+1)$ & $K(i)$ & $K(i+1)$ & $K(i)$ & $K(i+1)$ \\
\hline 1 & 1 & 8.706837 & 2 & 4.197874 & 3 & 2.725345 & 4 & 2.035211 \\
\hline 2 & 8.706837 & 1.250435 & 4.197874 & 1.944241 & 2.725345 & 3.016747 & 2.035211 & 4.120408 \\
\hline 3 & 1.250435 & 6.897759 & 1.944241 & 4.326401 & 3.016747 & 2.70944 & 4.120408 & 1.978533 \\
\hline 4 & 6.897759 & 1.358366 & 4.326401 & 1.890822 & 2.70944 & 3.035577 & 1.978533 & 4.246483 \\
\hline 5 & 1.358366 & 6.324136 & 1.890822 & 4.456776 & 3.035577 & 2.691786 & 4.246483 & 1.923543 \\
\hline 6 & 6.324136 & 1.420669 & 4.456776 & 1.840735 & 2.691786 & 3.056757 & 1.923543 & 4.376043 \\
\hline 7 & 1.420669 & 6.032818 & 1.840735 & 4.586002 & 3.056757 & 2.672215 & 4.376043 & 1.871283 \\
\hline 8 & 6.032818 & 1.460676 & 4.586002 & 1.794821 & 2672215 & 3.080584 & 1.871283 & 4.506351 \\
\hline 9 & 1.460676 & 5.858008 & 1.794821 & 4.710089 & 3.080584 & 2.650554 & 4.506551 & 1.822699 \\
\hline 10 & 5.858008 & 1.487841 & 4.710889 & 1753609 & 2.650554 & 3.107394 & 1.822699 & 4.634299 \\
\hline 20 & 5.548741 & 1.543403 & 5.185799 & 1.622172 & 2.504601 & 3.300865 & 1.652229 & 5.143535 \\
\hline 30 & 5.486207 & 1.555836 & 5.385676 & 1.576777 & 2.284046 & 3.642588 & 1.585917 & 5.371494 \\
\hline 40 & 5.471277 & 1.558871 & 5.445342 & 1.564204 & 2.005357 & 4.185911 & 1.566622 & 5.441479 \\
\hline 50 & 5.46758 & 1.559626 & 5.461033 & 1.560968 & 1.757363 & 4.817671 & 1.561582 & 5.460044 \\
\hline 60 & 5.466657 & 1.559815 & 5.465013 & 1.560152 & 1.623655 & 5.239461 & 1.560306 & 5.464764 \\
\hline 70 & 5.466426 & 1.559862 & 5.466014 & 1.559947 & 1.577212 & 5.402853 & 1.559986 & 5.465951 \\
\hline 80 & 5.466368 & 1.559874 & 5.466264 & 1.559895 & 1.564318 & 5.449951 & 1.559905 & 5.466249 \\
\hline 90 & 5.46632 & 1.559877 & 5.466327 & 1.559883 & 1.560997 & 5.462208 & 1.559885 & 5.466323 \\
\hline 100 & 5.466349 & 1.559878 & 5.466343 & 1.559879 & 1.560159 & 5.465309 & 1.55988 & 5.466342 \\
\hline 1000 & 5.466348 & 1.559878 & 5.466348 & 1.559878 & 1.559878 & 5.466348 & 1.559878 & 5.466348 \\
\hline 1001 & 1.559878 & 5.466348 & 1.559878 & 5.466348 & 5.466348 & 1.559878 & 5.466348 & 1.559878 \\
\hline
\end{tabular}




\begin{tabular}{|c|c|c|c|c|c|c|c|c|}
\hline \multirow{2}{*}{$\begin{array}{c}\text { Iteration } \\
\#\end{array}$} & \multicolumn{2}{|c|}{$k=5$} & \multicolumn{2}{|c|}{$k=10$} & \multicolumn{2}{|c|}{$k=20$} & \multicolumn{2}{|c|}{$k=50$} \\
\hline & $K(i)$ & $K(i+1)$ & $K(i)$ & $K(i+1)$ & $K(i)$ & $K(i+1)$ & $K(i)$ & $K(i+1)$ \\
\hline 1 & 5 & 1.669334 & 10 & 1.176412 & 20 & 1.170701 & 50 & 1.169289 \\
\hline 2 & 1.669334 & 5.087693 & 1.215742 & 7.352177 & 1.170701 & 7.389628 & 1.169289 & 7.398942 \\
\hline 3 & 5.087693 & 1.646441 & 7.10383 & 1.321036 & 7.389628 & 1.318344 & 7.398942 & 1.317682 \\
\hline 4 & 1.646441 & 5.162693 & 1.340311 & 6.511902 & 1.318344 & 6.525857 & 1.317682 & 6.529293 \\
\hline 5 & 5.162693 & 1.627765 & 6.413642 & 1.398092 & 6.525857 & 1.396505 & 6.529293 & 1.396116 \\
\hline 6 & 1.627765 & 5.225456 & 1.409616 & 6.135374 & 1.396505 & 6.142708 & 1.396116 & 6.144508 \\
\hline 7 & 5.225456 & 1.612746 & 6.082613 & 1.445849 & 6.142708 & 1.444821 & 6.144508 & 1.444569 \\
\hline 8 & 1.612746 & 5.27699 & 1.453371 & 5.922233 & 1.444821 & 5.926673 & 1.444569 & 5.927761 \\
\hline 9 & 5.27699 & 1.600814 & 5.889944 & 1.477642 & 5.926673 & 1.476941 & 5.927761 & 1.476769 \\
\hline 10 & 1.600814 & 5.318627 & 1.482798 & 5.788011 & 1.476941 & 5.79091 & 1.476769 & 5.79162 \\
\hline 20 & 1.570683 & 5.426604 & 1.542361 & 5.535962 & 1.541167 & 5.5365 & 1.541133 & 5.536632 \\
\hline 30 & 1.562623 & 5.456201 & 1.555586 & 5.483227 & 1.555301 & 5.483353 & 1.555293 & 5.483384 \\
\hline 40 & 1.560568 & 5.463794 & 1.558809 & 5.470543 & 1.558738 & 5.470574 & 1.558736 & 5.470582 \\
\hline 50 & 1.560051 & 5.465708 & 1.559611 & 5.467397 & 1.559593 & 5.467405 & 1.559593 & 5.467407 \\
\hline 60 & 1.559922 & 5.466188 & 1.559811 & 5.466611 & 1.559807 & 5.466613 & 1.559807 & 5.466613 \\
\hline 70 & 1.559889 & 5.466308 & 1.559862 & 5.466414 & 1.55986 & 5.466415 & 1.55986 & 5.466415 \\
\hline 80 & 1.559881 & 5.466338 & 1.559874 & 5.466365 & 1.559874 & 5.466365 & 1.559874 & 5.466365 \\
\hline 90 & 1.559879 & 5.466346 & 1.559877 & 5.466352 & 1.559877 & 5.466352 & 1.559877 & 5.466352 \\
\hline 100 & 1.559878 & 5.466348 & 1.559878 & 5.466349 & 1.559878 & 5.466349 & 1.559878 & 5.466349 \\
\hline 1000 & 1.559878 & 5.466348 & 1.559878 & 5.466348 & 1.559878 & 5.466348 & 1.559878 & 5.466348 \\
\hline 1001 & 5.466348 & 1.559878 & 5.466348 & 1.559878 & 5.466348 & 1.559878 & 5.466348 & 1.559878 \\
\hline
\end{tabular}

\section{ACKNOWLEDGEMENT}

The author is thankful to the Meteorological Office Karachi for providing us with the wind data for this study.

\section{REFERENCES}

[1] Baños, R.; Manzano-Agugliaro, F.; Montoya, F.G.; Gil, C.; Alcayde, A.; Gómez, J. Optimizationmethods applied to renewable and sustainable energy: A review. Renew. Sustain. Energy Rev. 15 (2011).

[2] Acker, T.L.; Williams, S.K.; Duque, E.P.; Brummels, G.; Buechler, J. Wind resource assessmentin the state of Arizona: Inventory, capacity factor, and cost. Renew. Energy, 32, 1453-1466.

[3] Cruz-Peragon, F.; Palomar, J.M.; Casanova, P.J.; Dorado, M.P.; Manzano-Agugliaro, F.Characterization of solar flat plate collectors. Renew. Sustain. Energy Rev. 2012, $16,1709-1720$.

[4] Claire Vincent, Gregor Giebel, Pierre Pinson and Henrik Madsen, "Resolving Nonstationary Spectral Information in Wind Speed Time Series Using the Hilbert-Huang Transform", J. Appl. Meteor. Climatol,.2010, 49(2), 253-267.

[5] LipmanN. H., BossanyiE. A., DunnP. D., MusgroveP. J., WhittleG. E., and C. Maclean;"Fluctuations in the output from wind turbine clusters", Wind Engineering. 1980, 4(1), 1-7.1980. 6.

[6] Akhmatov, V. Influence of wind direction on intense power fluctuations in large offshore wind farms in the North Sea. Wind Eng. 2007, 31, 59-64.

[7] Chen, Z.; Blaabjerg, F. Wind farm-A power source in future power systems. Renew. Sustain.Energy Rev. 2009, 13, 1288-1300.

[8] Poul Sørensen, Nicolaos Antonio Cutululis, Antonio Vigueras-Rodríguez, Henrik Madsen, Pierre Pinson, Leo E. Jensen, Jesper Hjerrild andMartin Donovan, 
"Modelling of power fluctuations from large offshore wind farms", Wind Energy 2008, 11 (1), 29-43.

[9] A.Z. Dhunny, M.R. Lollchund, R. Boojhawon and S.D.D.V. Rughooputh, "Statistical Modelling of Wind Speed Data for Mauritius", International journal of renewable energy research 2014, 4(4), 1056-1064.

[10] Ngala G.M., Alkali B. and Aji M.A., "Viability of wind energy as a power generation source in Maiduguri, Borno state, Nigeria". Renewable Energy. 2007, 32(13), 2242-2246.

[11] Cartaa JA, Ramírezb P, Velázquezc S. A review of wind speed probability distributions used in wind energy analysis:Case studies in the Canary Islands. Renewable andSustainable Energy Reviews 2009; 13(5): 933-955.

[12] Ucar A. and Balo F., "Investigation of wind characteristic and assessment of windgeneration

\section{AUTHORS}

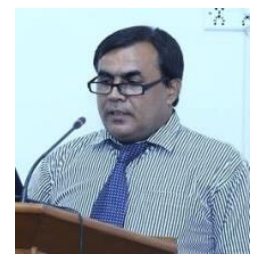

Fida Hussain Khoso

fida.dcet74@.com

Ph.D Scholar, Department of Computing, Faculty of Engineering, Science \& Technology (FEST), Indus University Karachi (Pakistan).

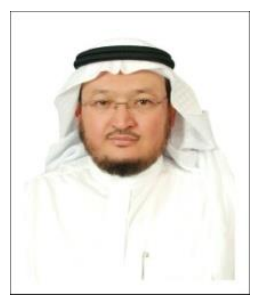

\section{Dr. Gasim Alandjani}

alandjanig@rcyci.edu.sa

Gasim Alandjani received his PhD Computer Engineering degree from New Mexico State University (USA), He has 28 years' experience of teaching and research including management experience as Dean, Makkah College of Technology-2003-2009, Deputy Managing Director of Yanbu Industrial College 2010-2012, managing Director of Yanbu Industrial College 20122013. Currently, he is working as senior faculty Member in Computer science and Engineering Department (CSE) at Yanbu University College Royal Commission Yanbu, Kingdom of Saudi Arabia.

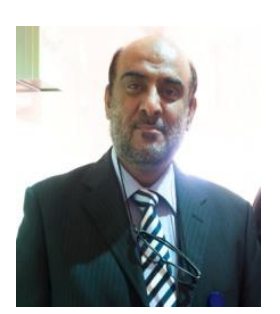

\section{Altaf Hussain Bouk}

bouka@rcyci.edu.sa

Altaf Hussain Bouk, earned his PhD from University of Parma Italy in 2005. HE became a Professor \& Chairman Dept of Computer Science and Technology, University of Balochistan, Quetta Pakistan. Since Sept 2008, he is working as Senior Lecturer Yanbu Industrial College as well as Yanbu University College, Yanbu Madina Munwarah, (Saudi Arabia).

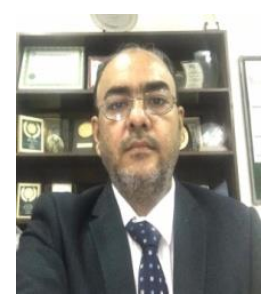

Prof. Dr. Engr. Sayed Hyder Abbas Musavi

dean@indus.edu.pk

Senior Member IEEE

D. Musavi earned his PhD Degree in 2011 in Telecommunication Engineering. He has 25 years of teaching and research experience. He is currently serving as Dean at Faculty of Engineering, Science \& Technology Indus University, Karachi, (Pakistan). 


\section{AUTO SPRUCE TRIAL SYSTEM (ASTS)}

Huma Hassan Rizvi

Computer Engineering Department Sir Syed University of Engineering and Technology, Karachi, (Pakistan)

E-mail: humahrizvi@yahoo.com

Sana

Software Engineering Department Sir Syed University of Engineering and Technology, Karachi, (Pakistan)

E-mail: raosaleem770@gmail.com

Dr. Sadiq Ali khan

Department of Computer Science

University of Karachi, (Pakistan)

E-mail: msakhan@uok.edu.pk

Muhammad Khurrum

Department of Informatics

Malaysia university of science \& technology, (Malaysia)

E-mail: muhammad.khurram@pg.must.edu.my

Khalique Ahmed

Computer Engineering Department

Sir Syed University of Engineering \& Technology, Karachi, (Pakistan)

E-mail:kkhanzada@hotmail.com 


\section{ABSTRACT}

Auto Spruce Trial System (ASTS) is designed to provide a platform in which children's intelligence and cognitive behavior are tested through Wechsler Intelligence Scale for Children (WISC). This test is used for a children assessment and find out their abilities learning and disabilities, as well as a clinical device. ASTS system is an automated testing system which conducts the children test and generates their intelligence result automatically. We automate the system as mentioned above in Pakistan which is taken manually and consumes a lot of time. It does not really matter how much intelligence one has, what makes a difference is the manner by which well one uses his/her intelligence. This test is applicable for those children's whose parents are worried about their mental health's issues and their learning potential. An intelligence test can encourage guardians and instructors make judgments around an individual child's educational course, standard, or in need of special education.

\section{KEYWORDS}

Wechsler Intelligence Scale for Children (WISC).

\section{INTRODUCTION}

This testing system as mentioned above, developed by David Wechsler. It's a separately directed intelligence test for children between the ages of six and sixteen. The original test as mentioned above was developed in 1939, and this test divided into several of the subtests. The subtest was arranged into Verbal and Performance scales. These test scores based on:

- Verbal IQ (VIQ)

- Performance IQ (PIQ)

- Full Scale IQ (FSIQ)

The third edition was published in 1991 named as WISC-III. This edition has introduced a new subtest as a measure of processing speed. These four new index scores were introduced to represent more narrow domains of cognitive function:

- Verbal Comprehension Index (VCI)

- Perceptual Organization Index (POI)

- Freedom from Distractibility Index (FDI)

- Processing Speed Index (PSI)

The WISC-IV and WISC-V are published in the year 2003 and 2014 respectively. The WISC-V has included a total of 21 subtests which based on 15 composite scores [2].

In this paper we talk about a sort of use named as ASTS which can assist guardians with making a brilliant and prosperous future for their kids.

The application ASTS can be utilized in schools for special children's. ASTS is used not only as an intelligence test, but it is also used for other indicative purposes. IQ scores detailed by the ASTS and these outcomes can be utilized as a component to diagnose the children mental retardation and specific learning disabilities. But, here in this application we are just focusing on how to find out the cognitive functioning of a child. 


\subsection{Purpose}

ASTS is quite different from others. It is more reliable than any other system and Institutionalized knowledge tests are developed by strict rules to guarantee unwavering quality and legitimacy. This test is reliable when achieved a desired outcome. The main purpose of this system is to provide guidance to the parents who's really concerned about their children's mental health issues, so it will be a good approach to create something new and more reliable.

\subsection{Scope}

Since, we know that it is a fact that everybody wants a new idea or something innovative. Auto Spruce Trial System (ASTS) is designed for the diagnosis of the Intelligence Quotient (IQ) level of a child and will be able to predict the presence of disorder in the children based on the age and number of answered question in a specific time and patterns based on the scaling system. By using ASTS, we can easily determine the cognitive functioning of any child. This testing system as mentioned above is applicable for children from age six to sixteen years. This test is utilized as an intelligence test, as well as a clinical apparatus. This project utilizes all our work, academic skills and our experience to making a remarkable source for us to learn more things and grow more into this field.

\subsection{Modules of auto spruce trial system}

Our system consists of 3 modules, as follows:

Module-1: Pre-designed Testing System

Module-2: Consultancy

Module-3: Bulletin Board

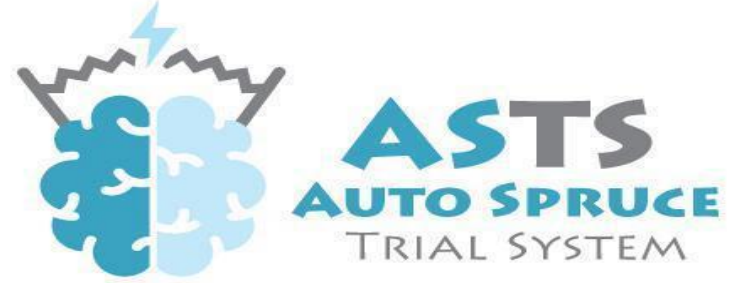

Figure 1. Logo of our application.

\section{Module-1: Pre-designed Testing System}

In this module, we simply automate the testing system as mentioned above it's allow the physiatrist to identify the stages of mind development. It has four main indexes. These indexes are below:

i) Verbal Comprehension Index (VCI)

ii) Perceptual Reasoning Index (VRI)

iii) Working Memory Index (WMI)

iv) Processing Speed Index (PSI)

There are a variety of subtests within each of these indexes[3].

1. The VCI Score test your child's intelligence and knowledge. The subtest includes:

- Vocabulary, Similarities, Comprehension, Information*, Word Reasoning*

2. The PRI scores is related to intelligence and ability to learn new information. The subtest includes:

- Block Design, Matrix Reasoning, Picture Concepts, Picture Completion* 
3. WMI score is related to short term memory. The subtest includes:

- Forward Digit Span, Backward Digit Span, Letter-Number Sequencing, Arithmetic*

4. PSI test focuses on mental quickness and task performance, its mainly concerned with concentration and attention. The subtest includes:

- Coding, Symbol Search, Cancellation* [3].

\section{Module-2: Consultancy}

It provides a consultancy section. Everyone who gives test, do not understand their test result and for their easiness we will provide consultants who will guide them online and evaluate their personality perfectly.

\section{Module-3: Bulletin Board}

Bulletin Board includes a portion where users can view updates about a particular issue or topic. It is a surface intended for the posting of public messages. It displays the daily updates of a website. If anything new happens, will be shown at the bulletin board.

\section{LITERATURE REVIEW}

Auto spruce trial system is an automated intelligence testing system which is based on the real and authorized data bank set. Automated System and websites exists, that conduct WISC-IV testing but their data set is not appropriate and according to the measures of testing system as mentioned above credibility. ASTS applies accurate and authenticate data set based on level of WISC-IV. ASTS is quite different from others. ASTS, is more reliable than any other system and standardized intelligence tests. ASTS is built by strict rules to ensure reliability and validity. A test result is considered reliable if we can get the equivalent/comparative outcome over and over.

ASTS is designed to overcome the difficulties of psychologists when they take manual tests and generate their result in few days. The difficulties, they face are:

1. The test is taken manually.

2. The result is generated lately.

3. Proper time is not given to individuals.

\subsection{Old methods used}

\section{A Test Sheet Algorithm for Assessments}

A dynamic programming approach is used to solve the problem generated by multiple criteria test-sheet. This utilizes the techniques of clustering and dynamic programming that allows the construction of a possible test sheet in accordance with the specified requirements. In this paper some experimental results and the testsheet-generating strategy of ITED is discussed to evaluate the efficiency of the approach [5].

\section{Fuzzy Logic-based Student Learning Assessment Model}

In this article a diagnosis model based on fuzzy logic has been presented. One of the main advantages of this model is that it sanctions for a representation of interpret able cognizance since it is predicated on rules when the reasoning is well defined as well as when the reasoning is intuitive, as a result of experience. The qualitative and quantitative criteria in student assessment proposed by the teachers 
can be easily improved (linguistic variables as well as fuzzy rules) adding a high degree of flexibility [11].

In Development of Computer-Assisted Testing System with Genetic Test Sheet technique is followed [12]. They proposed two genetic algorithms:

- CLFG

- FIFG

Above techniques are used for test sheet-generating problems. In a less time by applying these approaches we can get the test sheets with near-optimal discrimination degrees. The two algorithms have been embedded in a CAI system, Intelligent Tutoring, Evaluation, and Diagnosis that provides the easiness and the more informative tool for the instructors an learners. The (ITED-II) testing sub systems generate the test sheets by accepting the assessment requirements by reading the test items from the item banks. In the end the test results are sending to the tutoring sub system for the arrangements of adaptive subject materials [12].

\section{Generation Algorithm for Test Sheet Results}

The test sheet generating issues are solved through an adaptive cellular genetic algorithm, which is based on selection strategy. This algorithm is a combination of Adaptive Test Sheet Generation and cellular genetic algorithm. This approach resolves the problems of test sheet generation space, improves the fitness of test sheet and also improves the assessment of child's. These techniques also improve the accuracy in calculations and convergence speed of calculations in test sheet generation [13].

\section{An Evolutionary Intelligent Water Drops Approach for Intelligence Test sheet Results Generation}

In this paper, an intelligence test sheet result generating problems and issues are resolved. The computerized test sheet results with multiples assessments and calculations are one of the major issues in the Computer Assisted Testing System an E-Learning technology. A huge and verity of different tests, questions and task banks with different abilities are involved in the assessments test, even randomized test cannot serve the purpose of assessment and cannot generate an accurate output. The accurate result of the system is based on correct question bank and algorithm. It is difficult to develop the assessment sheet that satisfies the all assessment criteria. Evolutionary Intelligent Water Drops is best and more suitable algorithm which solves all the issues related to test sheet results and also solve the huge amount of question bank assessments test [14].

\section{Genetic Algorithm used for assessment test}

In this research, genetic algorithm approach is used for genetic assessment test. This method is used for optimized the sequences multiple variety and group of tests which have used for same purpose and it is also use a less amount of hardware resources for optimal solutions. These tests are time consuming and some restrictions are applied. In this approach representative keywords used for a particular test. This approach has three major elements:

- Teaching

- Learning 
- Evaluation

The genetic algorithm helps in finding the best appropriate solutions [15].

The reference is given below of a report that is a result of testing tool kit as mentioned above of a child it describes all the tables that are used for performance evaluation [1][4].

\section{METHODOLOGY}

\subsection{Method/Technique}

The main motive of our development is to produce precise and trustworthy results. We don't have the right to ruin anyone's life as it is a matter of very serious problem, therefore the results of the system must be reliable. There have been many approaches that drive different results based on decisions that are made on different states. In every stage, a decision which achieves a reward closer to the total rewards is desirable. The new approach adopts fuzzy logic theory to diagnose the difficulty level of test items, in accordance with the learning status and personal features of each student, and then applies the techniques to the test sheet construction. Clustering and dynamic programming is also an approach to solve such issues. ASTS system is an automated testing system which conducts the children test and generate their intelligence result automatically in which we will apply fuzzy logic instead of clustering techniques and dynamic programming approach. We automate the testing system as mentioned above in Pakistan which is taken manually and consumes a lot of time. [4] ASTS will serve as an intelligent assistance to psychologist. The fuzzy logic will make the system more efficient and time saving and it will also become very helpful for the psychologist.

\subsection{Product Pers pective}

The system Auto Spruce Trial System (ASTS) is designed for the diagnosis of the Intelligence Quotient (IQ) level of a child and will be able to predict the presence of disorder in the children based on the age and number of answered question in a specific time and patterns based on the scaling system or the implementation of algorithm that are to be decided. The scores are cross matched with the scaled scores, composite scores, percentile rank or algorithm and provide the result in the terms of perfection or disorder. Type, kind, level and seriousness of disorder will be further provided in the consultancy section if required.

\subsection{System Functions}

The functions of the system are as follows:

- Generation of questions from question bank.

- Make record for the answered question.

- Compare the answers by the engine and perform calculations.

- Predict the IQ level.

- Predict disorder in the child if present on behalf of his/her answers.

\subsection{User View}

- User must know about this application features and a basic knowledge to operating the internet. 
- User of this is generally the children that will interact so only their proper attention is required.

- This system can also be used by parents/system for the result tracking of the child so; they should have basic knowledge of computer.

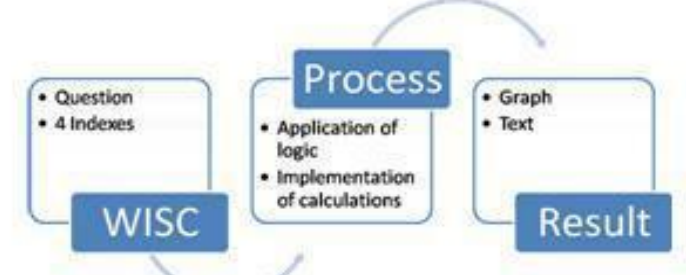

Figure 2. Abstract view of system.

\subsection{Operating Environment}

This is a web and android based system and hence will require a good GUI for good results. The basic need is the browser version for web users and android version for android user.

\subsection{Constraint}

- Expertise of members in the software used can be a constraint for the timely completion of the system.

- Inappropriate working of database and interface may be a constraint.

- Internet connection is important to run the function of the application.

- Database is shared between both web and mobile application it may be forced to queue incoming requests and therefore increase the time it takes to fetch data.

\subsection{System Assumption}

- Great amount of memory is required in cell phones to use this system.

- If your cell phone is not supporting well in memory and proper hardware resources so you can't access this system.

\subsection{User Role}

- First step is the users register him/her self into the system.

- The second step is system provides the access key to users.

- The third step is according to the age level of user the system starts to show the test questions in order to take test of user then user start to give the test.

- Next step is when user completed the test the system will show the test results. The result is in percentage form which determines the level of user's intelligence and their cognitive abilities.

- The last step is the user logout from the system.

\subsection{Overall System working through Diagram}

Overall working of the system through diagram is as follows: 


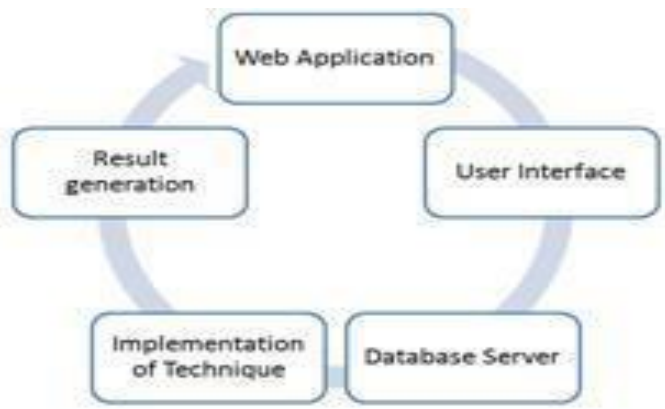

Figure 3. Web view of system.

Figure 4. android view of system.

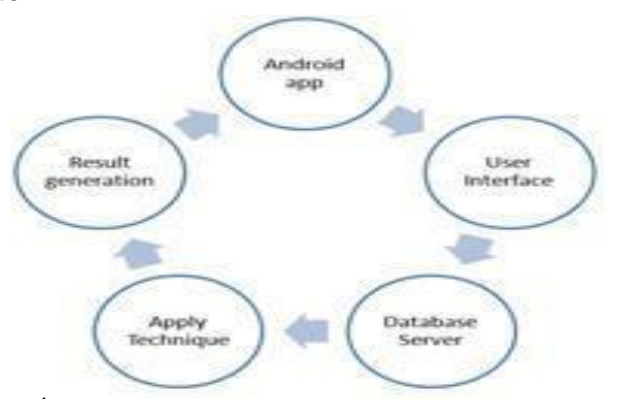

\subsection{Hardware and Software}

\section{Hardware}

- Laptop Mobile

- Operating Systems

\section{Windows Android}

- Databases

- MySql SQLite

\section{Programming Languages}

- Java PHP

- HTML5 CSS3

- Bootstrap

- JSON

\section{RESULTS AND DISCUSSIONS}

We automate the testing system as mentioned above in Pakistan which is taken manually and consumes a lot of time. Psychologists complete this test in $2 / 3$ days or in an entire week period of time. They cannot take this test continuously because neither they can concentrate on a test after 2 nor 3 hours nor children will be able to give test continuously.

The manually system is totally converted into automated system. It stands and outmost the credibility level of all available websites conducting WISC-IV tests. Its output/result will be a score sheet and recommendations paper of child's intelligence for parents and Psychologists. 


\section{Comparison:}

\begin{tabular}{|c|c|c|c|c|}
\hline Websites & Reliable & Correct & Usability & Authentic \\
\hline Assessment.com[6] & No & Yes & No & No \\
\hline Quiznatic.com[7] & No & Yes & No & No \\
\hline Fundaction.com[8] & No & No & No & No \\
\hline Brainmetrix.com[9] & No & No & No & No \\
\hline Asts4childern.com[10] & Yes & Yes & Yes & Yes \\
\hline
\end{tabular}

Table 1. Comparison of ASTS system with other systems $[6,7,8,9,10]$.

The comparison can be easily understandable through this chart:
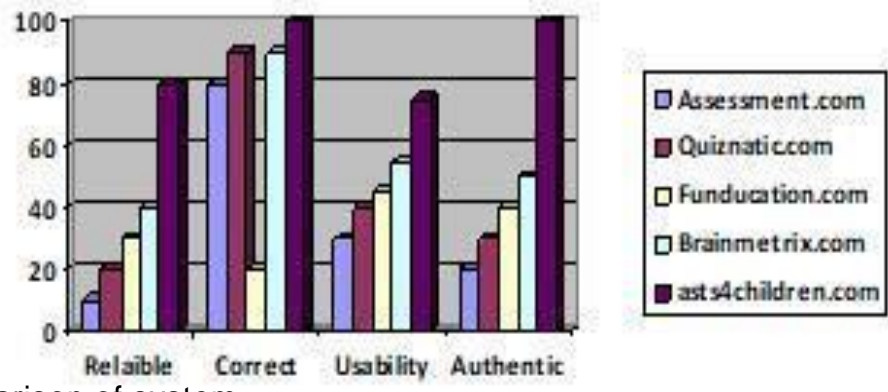

Figure 5. Comparison of system.

\section{CONCLUSION}

In this research, we have presented an application by which psychologists can take their assessment test easily. They don't have to wait for the entire week for result generation. Even, they don't have to do calculations on their own. All they have to do is to check the behavior of the children and help them through queries if they are stuck. The rest of the work will be done by the system itself, which includes calculations, displaying questions and results generation. This will help kids in their primary ages, when they are studying. Not only kids but parents who are concerned about their children will get benefit too. The system will let parents know about their child weaknesses and IQ. Not only this, parents can also consult with the consultants about how to increase their children IQ and what should be done and what shouldn't be done. This will help children to make their future bright and prosperous. In future, this application can be made more user friendly by implementing different GUI. For now, this application is only FYPs demonstration. But, after interacting with superior psychologist if they allow this application to be used for clinical purpose then we will implement it in clinics. And, we will not stop here, sooner it will be implemented in Schools and other educational institutions.

\section{REFERENCES}

\subsection{Patent}

[1] A WISC Descriptive and Graphical Report by Michelle C. Rexach, Licensed School Psychologist, Florida Department of Health.

\subsection{Websites}

[2] http://www.school-psychology.com.au/blog/wechslerintelligence-scale-forchildren-wisc-iv/

[3] https://www.helloq.com/overview/the-q-interactive-library/wisc-iv.html 


\subsection{Conference Proceedings}

[4] Anne-Marie Kimbell, "An Overview of the WISC “, Ph.D. National Training Consultant Pearson, 2015.

[5] Gwo-Jen Hwang, "A Test-Sheet-Generating Algorithm for Multiple Assessment Requirements”, ieee transactions on education, vol. 46, no. 3, august 2003.

\subsection{Websites}

[6] http://assessment.com/

[7] http://www.quiznatic.com/

[8] http://funducation.com/

[9] http://brainmetrix.com/

[10] http://asts4children.com/

\subsection{Research paper}

[11] Constanza Huapaya1, " Proposal of Fuzzy Logic-based Students Learning Assessment Model".

[12] Gwo-Jen Hwang, Bertrand M. T. Lin, Hsien-Hao Tseng, and Tsung-Liang Lin, "On the Development of a Computer-Assisted Testing System With Genetic Test Sheet-Generating Approach", ieee transactions on systems, man, and cyberneticspart c: applications and reviews, vol. 35, no. 4,november 2005.

\subsection{J ournal Article}

[13] Ankun Huang, Dongmei Li1, Jiajia Hou ,Tao Bi, "An Adaptive Cellular Genetic Algorithm Based on Selection Strategy for Test Sheet Generation", International Journal of Hybrid Information Technology, Vol.8, No.9 (2015).

[14] Kavitha, "Composition of Optimized Assessment Sheet with Multi-criteria using Evolutionary IntelligentWater Drops (EvIWD) Algorithm”, International Journal of Software Engineering and Its Applications, Vol. 10, No. 6 (2016).

[15] Doru Popescu Anastasiu, Nicolae Bold, and Daniel Nijloveanu, "A Method Based on Genetic Algorithms for Generating Assessment Tests Used for Learning",vol. 54, 2016, pp. 53-60. 


\section{TOOL PATH OPTIMIZATION OF A 3D PRINTER VIA AN ENHANCED ELECTROMAGNETISM-LIKE MECHANISM ALGORITHM FOR SOLAR PANEL BRACKETS FABRICATION}

Jian-Ding Tan

Institute of Sustainable Energy, Universiti Tenaga Nasional, 43000 Kajang, Selango, (Malaysia)

E-mail: TJianDing@uniten.edu.my

Chin-Wai Lim

Institute of Sustainable Energy, Universiti Tenaga Nasional, 43000 Kajang, Selango, (Malaysia)

Siaw-Paw Koh

Institute of Sustainable Energy, Universiti Tenaga Nasional, 43000 Kajang, Selango, (Malaysia)

Sieh-Kiong Tiong

Institute of Sustainable Energy, Universiti Tenaga Nasional, 43000 Kajang, Selango, (Malaysia)

Ying-Ying Koay

Institute of Sustainable Energy, Universiti Tenaga Nasional, 43000 Kajang, Selango, (Malaysia) 


\section{ABSTRACT}

There is a growing consensus that $3 \mathrm{D}$ printing technologies will be one of the next major technological revolutions. Over the past few years, many studies and researches have been carried out to improve and enhance the functions and performance of the 3D printers. In this paper, a modified Electromagnetism-like Mechanism (EM) algorithm is proposed to search for the optimum printing path of a $3 \mathrm{D}$ printer. The machine is set to fabricate the bracket of a photovoltaic solar energy harvesting panel. Instead of randomly setting the search step size, the improved EM systematically fine-tunes the steps to search for the best printing path. The performance of the enhanced EM is benchmarked with the conventional EM and Genetic Algorithm (GA). The results presented in this paper shows that the modified EM outperformed all other optimization techniques in terms of time taken, distance traveled and overall convergence process. We can thus conclude that the proposed modified EM performs well in optimizing the path planning sequence of a $3 \mathrm{D}$ printer.

\section{KEYWORDS}

Three Dimensional Printer, Electromagnetism-Like Mechanism Algorithm, Photovoltaic Solar Panel.

\section{INTRODUCTION}

Rapid prototyping, or better known as the 3D printing technologies have progressively taken more attention in the manufacturing research around the world as the process has proven to be compatible with industrial manufacturing beyond Prototyping [1-3]. The 3D printing technology evolved during the mid-1980s when computing and control systems progressed [4]. 3D printing is a form of "build-up" manufacturing, where an object is constructed and fabricated by adding layer after layer of a particular material. This is different from the conventional "cut-off" fabrication, in which an object is carved out of a block of raw material [5]. The systems are usually associated with the Computer-Assisted Design (CAD) software to digitally model the objects to be printed. It is widely recognized that the $3 \mathrm{D}$ printer offers significant advantages in terms of design freedoms, mass customization, and co-creation [6-8].

The study on the 3D printing technology can be split into several divisions. Among others is the tool path planning [9]. A primary goal in planning the tool path is to avoid tool collision. Solving the optimization problem of tool path has an important role because reducing the time to perform one piece ultimately leads to a significant reduction in cost of the entire series of fabrication. Several reports can be found in the literature on the tool path planning and optimization. In [10], the authors employed a hybrid algorithm to reduce the length of the tool path. A mathematical model for calculating the processing time was developed. The algorithm finds an optimal tool path, which has a proven effect on the process productivity. Some optimization mechanism, such as Ant Colony Optimization algorithm [11-13], Genetic Algorithm (GA) and Hill-Climbing [14, 15] were also attempted in the optimization of the tool path.

This paper presents an enhanced Electromagnetism-Like Mechanism algorithm (EM) for the tool path optimization of a $3 \mathrm{D}$ printer. In this research, the $3 \mathrm{D}$ printer is set to fabricate a bracket for a photovoltaic solar panel. The breakdown of the paper can basically be divided into 4 major sections. The second section of the paper 
presents the implementation method of the EM algorithm and the proposed modification. The experimental results are shown in section 3, along with the analysis and some discussions. The final section of the paper offers the conclusion made from the research.

\section{ELECTROMAGNETISM-LIKE ME CHANISM ALGORITHM}

This section presents the implementation procedure of a global optimization algorithm known as Electromagnetic-like Mechanism (EM) in minimizing the traveling path of the 3D printer tool. The EM is a global optimization search mechanism proposed by Birbil and Fang in 2003 [16]. The EM imitates the attraction-repulsion mechanism of electromagnetic charges in the search for a global optimal solution. In the EM, all solutions are known as particles in the search space and the charge of each reflects the objective value of each. Particles with better objective attract other particles. Particles with worse objective values, on the other hand, repulse other particles so that they move away from the known bad solutions [17]. The magnitudes of the forces are in proportion to the objective values. The particles are then moved based on superposition theorem. Figure 2.1 shows an example of the forces applications.

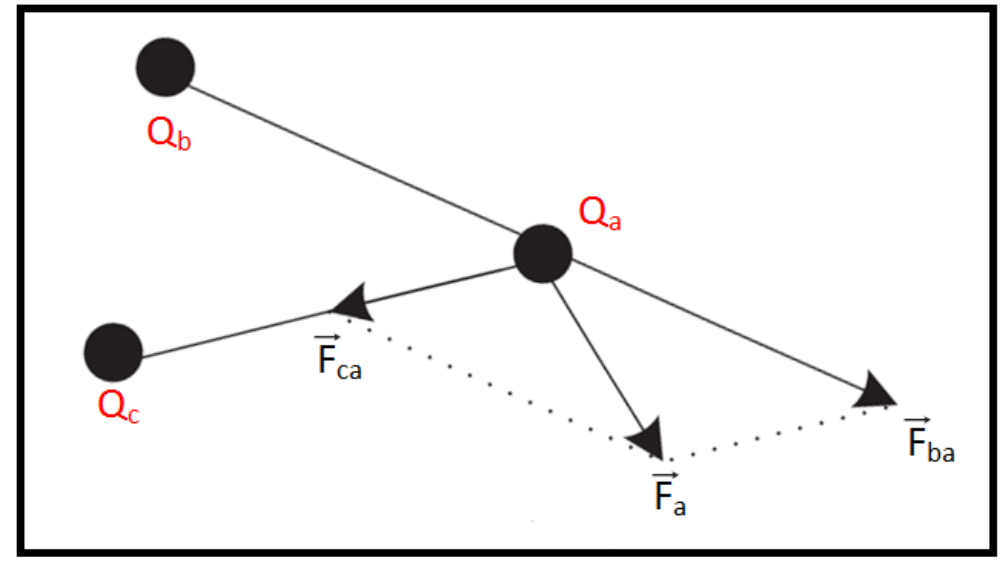

Figure 2.1. Total force exerted on $Q_{a}$ by $Q_{b}$ and $Q_{c}$.

There are five important operations in the EM, namely the initialization, the local search, the charge calculation, the force calculation, and the movement of particles. In the initialization stage, the feasible ranges of all the tuning parameters are defined. Then, $\mathrm{m}$ sample of initial particles are randomly picked from the feasible solution domain, each represents an $\mathrm{N}$ dimensional hyper-solid. Each value of dimension in each particle is assumed to be uniformly distributed inside the upper and lower bound. Since this research shows a minimization problem, the particle with the lowest tool travel path distance is marked as the best particle. Table 1 shows the pseudocode of the original EM proposed by Birbil and Fang in 2003 [16]. The mechanism is further illustrated in the flowchart shown in Figure 2.2.

Table 1. Original EM proposed by Birbil and Fang.

\begin{tabular}{|l|}
\hline EM $(m, M A X I T E R, L S I T E R, \delta)$ \\
\hline$m=$ number of initial particles \\
\hline$M A X I T E R:$ maximum number of iterations \\
\hline$L S I T E R:$ maximum number of local search iterations \\
\hline$\delta:$ local search parameter, $\delta \in(0,1)$ \\
\hline $1:$ Initialize ( ) \\
\hline
\end{tabular}




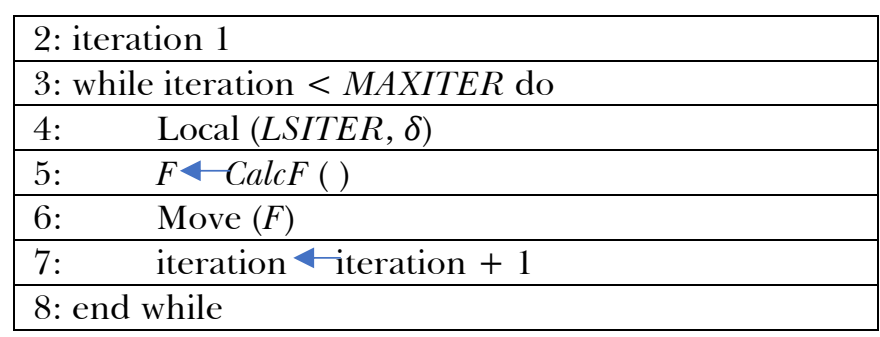

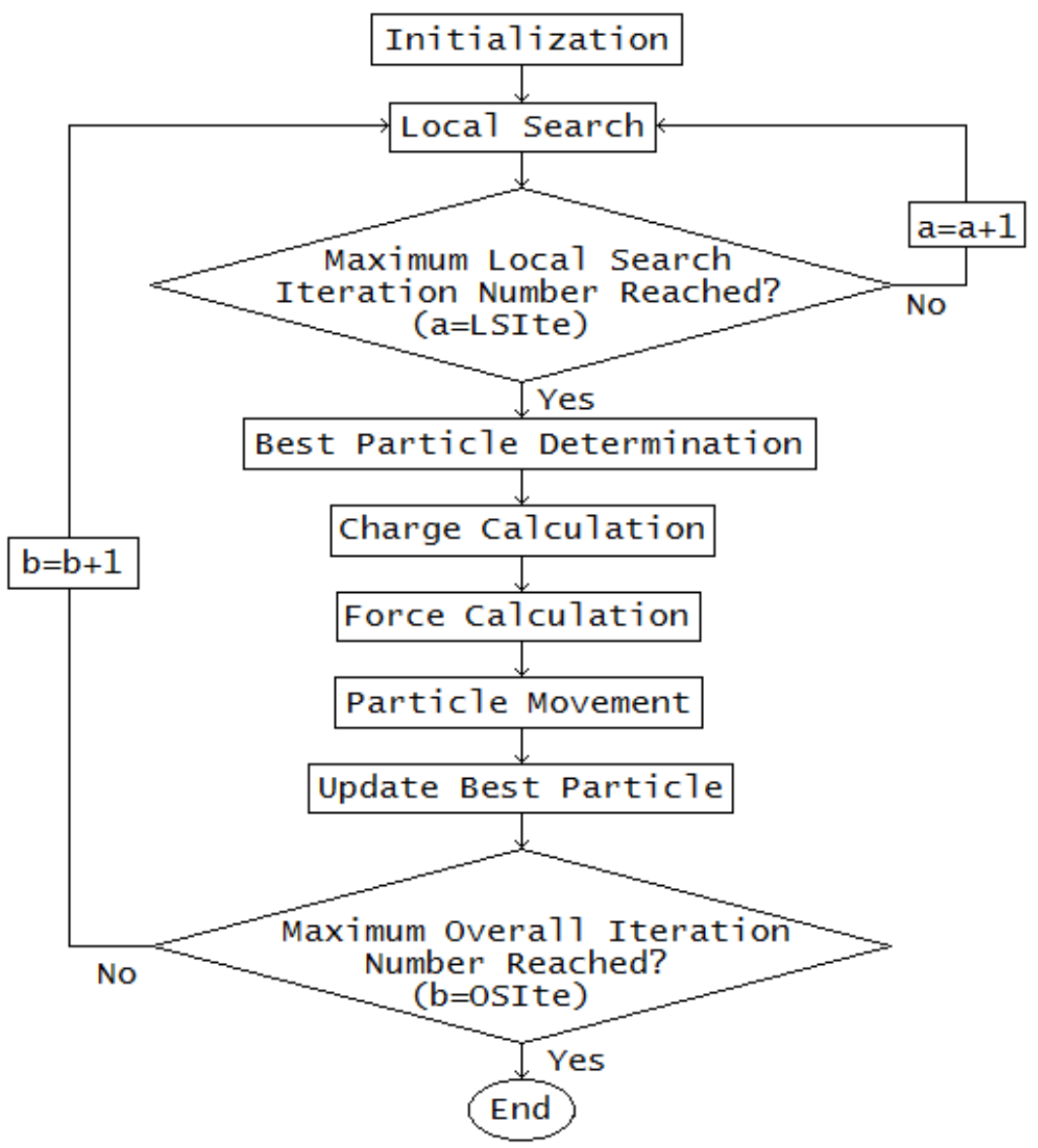

Figure 2.2. The mechanism flow of a conventional EM.

The original local search procedure in a conventional EM employs a random line search within the feasible range of a solution. This simple line search involves a particle being tuned along its dimensions one by one, restricted by a maximum feasible random step length of $\lambda \in(0,1)$. For each of the iterations, a new random step length is generated. The overall local search procedure is immediately terminated upon achieving any better objective value. Table 2 shows the pseudocode of the original local search procedure in the original EM. The flow of the original local search mechanism is as illustrated in Figure 2.3. 
Table 2. The original local search of the original EM.

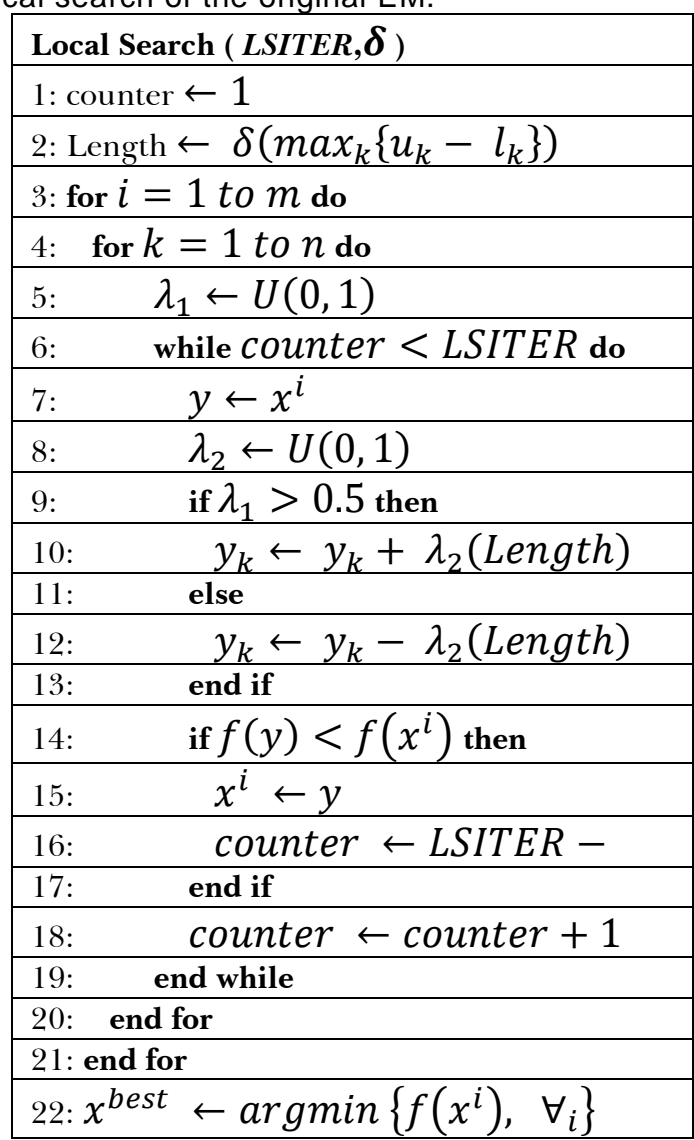

The total force vector exerted onto each particle is calculated based on the Coulomb's Law. The charge of each particle is evaluated by its current objective value compared to the best particle in the iteration. The computed charge of a particle, qi , when compared to that of other particles, will determine if it is a repulsive or attractive force to the respective particles. The calculation of qi is shown in equation (1):

$$
q^{i}=\exp \left(-n \frac{f\left(x^{i}\right)-f\left(x^{b e s t}\right)}{\sum_{k=1}^{m}\left(f\left(x^{k}\right)-f\left(x^{b e s t}\right)\right)}\right), \forall i
$$

where $\mathrm{n}$ refers the total dimension of the particle and $\mathrm{m}$ denotes the population size. $f(x b e s t)$ represents the objective value of the best particle.

With the charges calculated for all particles, forces generated by one particle onto another can be computed. According to the electromagnetic theory, the force of one particle onto another is inversely proportional to the distance between the two particles and directly proportional to the product of their charges. The force vector for a particle can be determined using equation (2).

$$
F^{i}=\sum_{j \neq i}^{m}\left\{\begin{array}{llll}
\left(x^{j}-x^{i}\right) \frac{q^{i} q^{j}}{\left\|x^{j}-x^{i}\right\|^{2}} & \text { if } & f\left(x^{j}\right)<f\left(x^{i}\right) \\
\left(x^{i}-x^{j}\right) \frac{q^{i} q^{j}}{\left\|x^{j}-x^{i}\right\|^{2}} & \text { if } & f\left(x^{j}\right) \geq f\left(x^{i}\right)
\end{array}\right\}, \forall i
$$

where $f(x j)<f(x i)$ denotes attraction and $f(x j) \geq f(x i)$ refers to repulsion. 


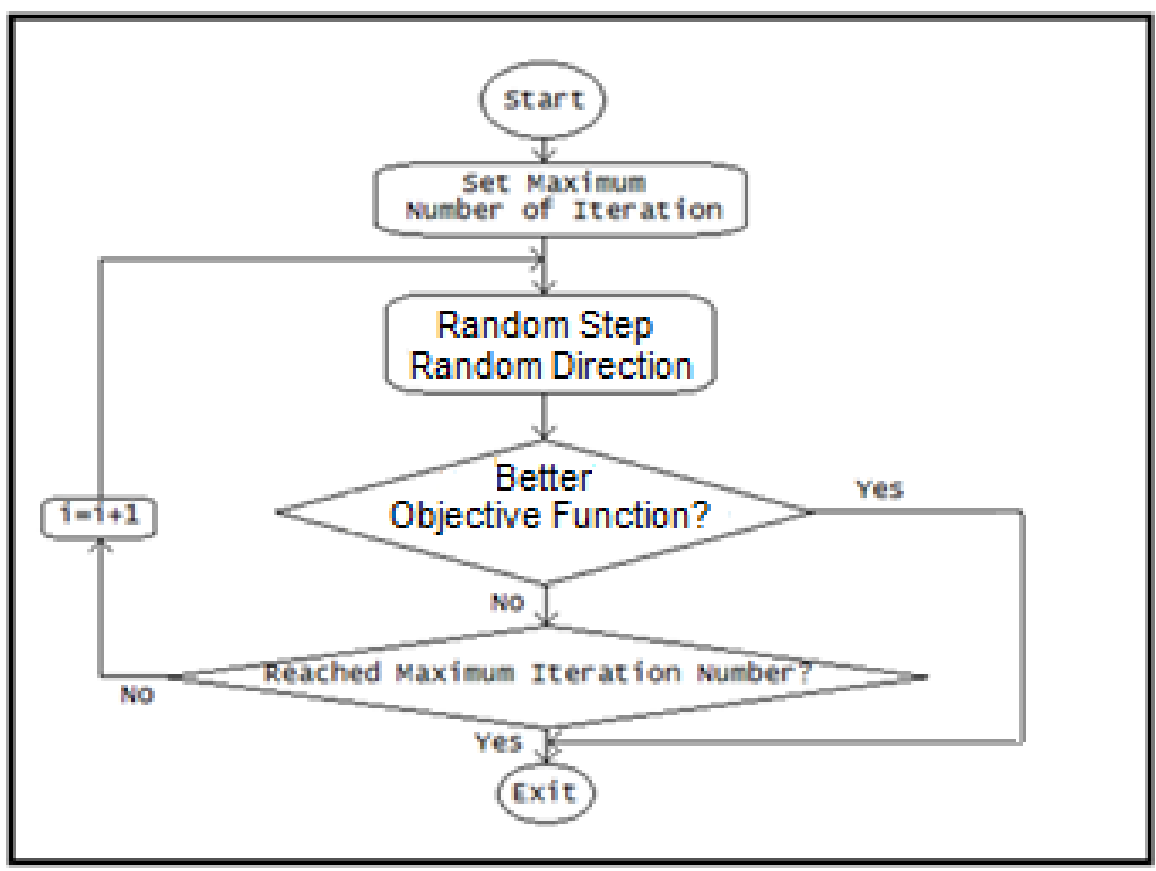

Figure 2.3. The flow of the original local search mechanism.

The movement stage in EM involves relocation of all particles but the best to a new location in space. This step is crucial to ensure better global exploration of other possible routes. The calculation for the movement of a particle is as shown in equations (3), where $\lambda$ represents the global particle movement step length. It is a random value between 0 and 1 , assumed to be uniformly distributed between the upper boundary (uk ) and the lower boundary (lk ).

$$
\begin{array}{lll}
x_{k}^{i} \leftarrow x_{k}^{i}+\lambda F_{k}^{i}\left(u_{k}-x_{k}^{i}\right) & ; F_{k}^{i} \geq 0 \\
x_{k}^{i} \leftarrow x_{k}^{i}+\lambda F_{k}^{i}\left(x_{k}^{i}-l_{k}\right) & ; F_{k}^{i}<0
\end{array}
$$

Holding the absolute power of attraction towards all other particles, the best particle of the iteration does not move. After a pre-fixed number of iterations, the best optimized tool path is then fed back as the result.

\subsection{The Enhanced EM}

In this research, a modification on the EM is proposed. This enhanced algorithm is named as Regulated Step EM (ReSEM). ReSEM is a modified version of EM that can grant the algorithm the ability to hit a more accurate result without heavily slowing down the entire convergence process. The idea of ReSEM is to replace the local search segment of a conventional EM with the procedure as shown in Table 3. ReSEM applies a nonlinear equation to dynamically adjust the step size as iterations go. The step $\lambda$ is calculated using equation (4).

$$
\lambda=\frac{1}{i+0.125}
$$

Figure 2.4 shows the modified workflow and decision making process of the enhanced ReSEM for the implementation in this research, where D represents the current solution in a particular dimension of an iteration and $\lambda$ refers to the search step calculated using equation (4). The flow systematically checks the direction and the magnitude of the convergence movement of each iteration and change accordingly. This ensure the algorithm search in relatively larger steps in the 
beginning of the iterations and move towards a more fine-tuned search as the iteration goes.

Table 3. Local search procedures for the proposed ReSEM.

\begin{tabular}{|l|l|}
\hline \multicolumn{2}{|l|}{ ReSEM Procedures } \\
\hline Step 1 & Set maximum number of iterations as terminating criteria. \\
\hline Step 2 & Calculate the step size according to equation 4. \\
\hline Step 3 & $\begin{array}{l}\text { Tune the current tile angle by adding and subtracting it with } \lambda \text { separately. } \\
\text { Calculate the new output power with the new solutions obtained. }\end{array}$ \\
\hline Step 4 & Adapt the newfound tilt angle value if a higher output power is achieved. \\
\hline Step 5 & Repeat Steps 3 and 4 until no higher output power is obtained. \\
\hline Step 6 & $\begin{array}{l}\text { Exit if the iteration number reaches termination criteria. Otherwise move on to } \\
\text { the next iteration }(i=i+1) \text { and repeat from Step } 2 .\end{array}$ \\
\hline
\end{tabular}

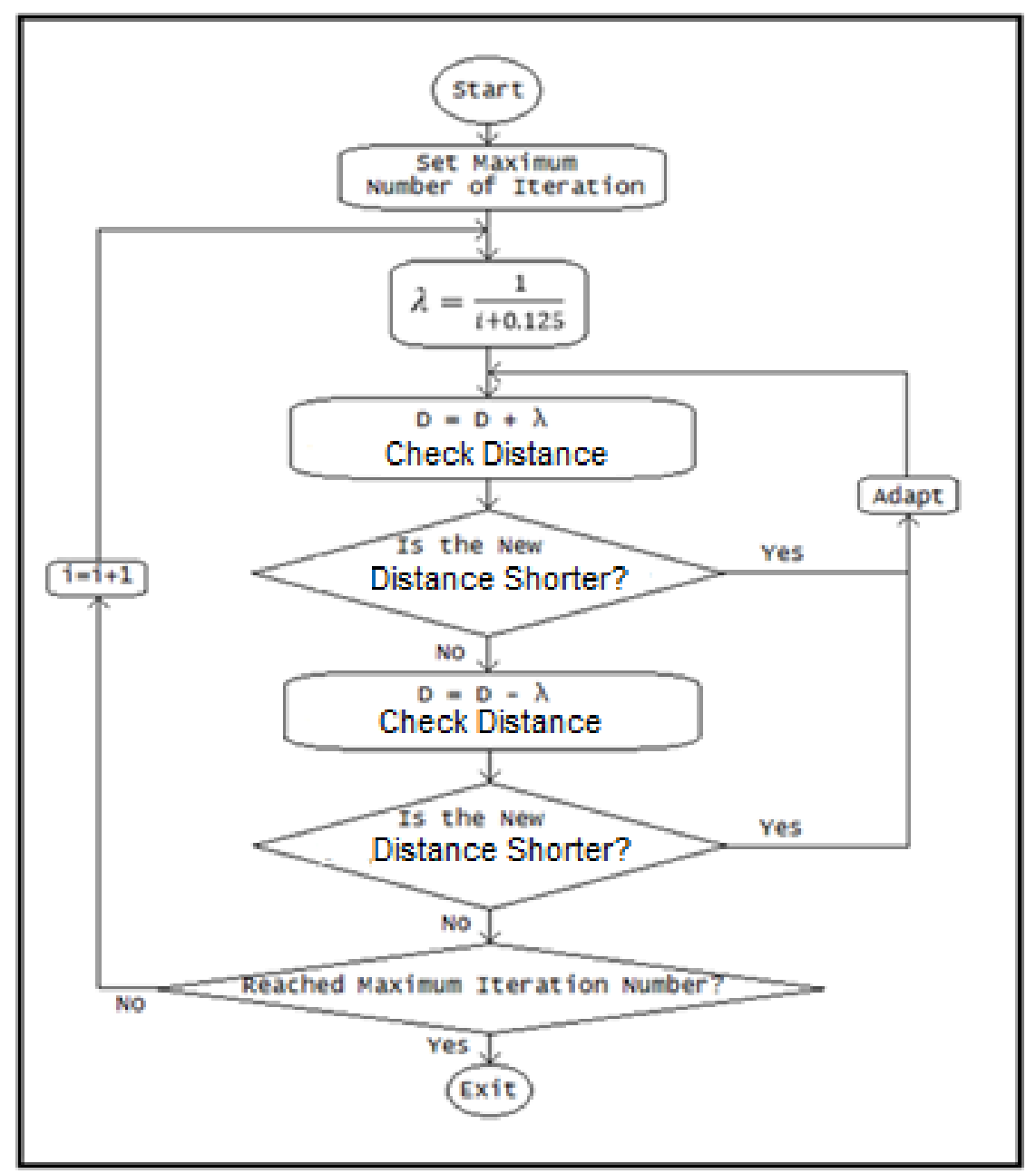

Figure 2.4. The proposed modification on the local search sequence of the modified EM.

The enhanced algorithm is then implemented to search for the shortest and quickest path in the simulation runs of a $3 \mathrm{D}$ printer to print the bracket. Figure 2.5 shows an example of the printing layers which the experiment is conducted on. The enhanced algorithm is set to search for the optimized tool path to print the shaded areas. 


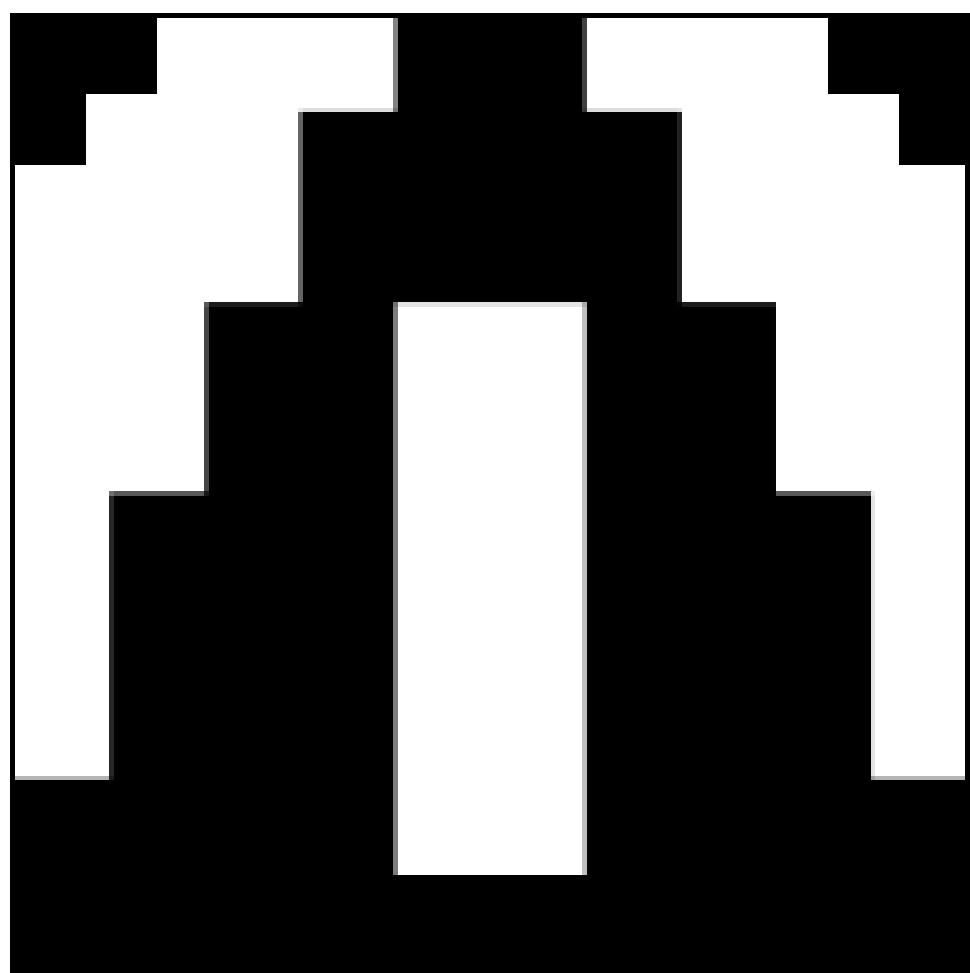

Figure 2.5. Example of the printing layers.

\section{EXPERIME NTAL RESULTS}

The performance of the enhanced EM is benchmarked with that of the conventional EM. Also, in order to show a better picture on the performance of the enhance EM compared to other optimization algorithms, a standard Genetic Algorithm (GA) is also included in the benchmarking process. Figure 3.1 shows the flow of the GA used in the benchmarking. The experiment is conducted in 10 individual runs for each of the algorithms. The results are shown in Table 4 in the form of best distance, worst distance, average distance, average time and average iteration needed to reach best optima results of the 10 individual runs.

From Table 4, it can be observed that the enhanced ReSEM shows relatively better results as it found shorter distance to travel compared to the conventional EM and the GA. Also, notice that this led to a shorter time needed to travel in order to complete the task.

In order to investigate the overall convergence performance of the algorithms, some convergence processes of the experiment are sampled and analyzed. The iterationby-iteration comparison of the sampled convergence processes are showed in Table 5. Figure 3.1 provides a better illustration of the iteration movement comparison of each algorithm.

Table 4. Results benchmarking.

\begin{tabular}{|l|l|l|l|}
\hline & ReSEM & Conventional EM & GA \\
\hline Best Distance & $1.267 \mathrm{~m}$ & $1.282 \mathrm{~m}$ & $1.315 \mathrm{~m}$ \\
\hline Worst Distance & $1.288 \mathrm{~m}$ & $1.495 \mathrm{~m}$ & $1.580 \mathrm{~m}$ \\
\hline Average Distance & $1.275 \mathrm{~m}$ & $1.391 \mathrm{~m}$ & $1.507 \mathrm{~m}$ \\
\hline Average Time & $92.7 \mathrm{~s}$ & $107.6 \mathrm{~s}$ & $114.8 \mathrm{~s}$ \\
\hline Average Iteration & 5.2 & 18.1 & 29.7 \\
\hline
\end{tabular}


Table 5. Comparison on the examples of the convergence process for ReSEM, Conventional EM and GA.

\begin{tabular}{|c|c|c|c|c|c|c|c|}
\hline Ite. & ReSEM & $\begin{array}{c}\text { Conv. } \\
\text { EM }\end{array}$ & GA & Ite. & ReSEM & $\begin{array}{c}\text { Conv. } \\
\text { EM }\end{array}$ & GA \\
\hline 0 & 3.975 & 3.21 & 2.981 & 26 & 1.27 & 1.387 & 1.427 \\
\hline 1 & 2.352 & 2.89 & 2.875 & 27 & 1.27 & 1.387 & 1.427 \\
\hline 2 & 1.599 & 2.67 & 2.769 & 28 & 1.27 & 1.387 & 1.427 \\
\hline 3 & 1.416 & 2.53 & 2.663 & 29 & 1.27 & 1.387 & 1.427 \\
\hline 4 & 1.355 & 1.899 & 2.502 & 30 & 1.27 & 1.387 & 1.427 \\
\hline 5 & 1.283 & 1.899 & 2.434 & 31 & 1.27 & 1.387 & 1.427 \\
\hline 6 & 1.27 & 1.899 & 2.105 & 32 & 1.27 & 1.387 & 1.427 \\
\hline 7 & 1.27 & 1.471 & 1.988 & 33 & 1.27 & 1.387 & 1.427 \\
\hline 8 & 1.27 & 1.471 & 1.986 & 34 & 1.27 & 1.387 & 1.427 \\
\hline 9 & 1.27 & 1.462 & 1.984 & 35 & 1.27 & 1.387 & 1.427 \\
\hline 10 & 1.27 & 1.453 & 1.982 & 36 & 1.27 & 1.387 & 1.427 \\
\hline 11 & 1.27 & 1.453 & 1.98 & 37 & 1.27 & 1.387 & 1.427 \\
\hline 12 & 1.27 & 1.4 & 1.978 & 38 & 1.27 & 1.387 & 1.427 \\
\hline 13 & 1.27 & 1.4 & 1.806 & 39 & 1.27 & 1.387 & 1.427 \\
\hline 14 & 1.27 & 1.4 & 1.793 & 40 & 1.27 & 1.387 & 1.427 \\
\hline 15 & 1.27 & 1.4 & 1.503 & 41 & 1.27 & 1.387 & 1.427 \\
\hline 16 & 1.27 & 1.399 & 1.486 & 42 & 1.27 & 1.387 & 1.427 \\
\hline 17 & 1.27 & 1.387 & 1.481 & 43 & 1.27 & 1.387 & 1.427 \\
\hline 18 & 1.27 & 1.387 & 1.481 & 44 & 1.27 & 1.387 & 1.427 \\
\hline 19 & 1.27 & 1.387 & 1.481 & 45 & 1.27 & 1.387 & 1.427 \\
\hline 20 & 1.27 & 1.387 & 1.481 & 46 & 1.27 & 1.387 & 1.427 \\
\hline 21 & 1.27 & 1.387 & 1.452 & 47 & 1.27 & 1.387 & 1.427 \\
\hline 22 & 1.27 & 1.387 & 1.45 & 48 & 1.27 & 1.387 & 1.427 \\
\hline 23 & 1.27 & 1.387 & 1.446 & 49 & 1.27 & 1.387 & 1.427 \\
\hline 24 & 1.27 & 1.387 & 1.44 & 50 & 1.27 & 1.387 & 1.427 \\
\hline 25 & 1.27 & 1.387 & 1.43 & & & & \\
\hline & & & & & & & \\
\hline
\end{tabular}

It can be observed from Figure 3.2 that the modified ReSEM shows rapid convergence process at the beginning of the iterations. Relatively, the conventional EM and the GA show slower convergence rates. The enhanced ReSEM also manages to hit lower distance value compared to that of the conventional EM and the GA. convergence processes are showed in Figure 3.2. 


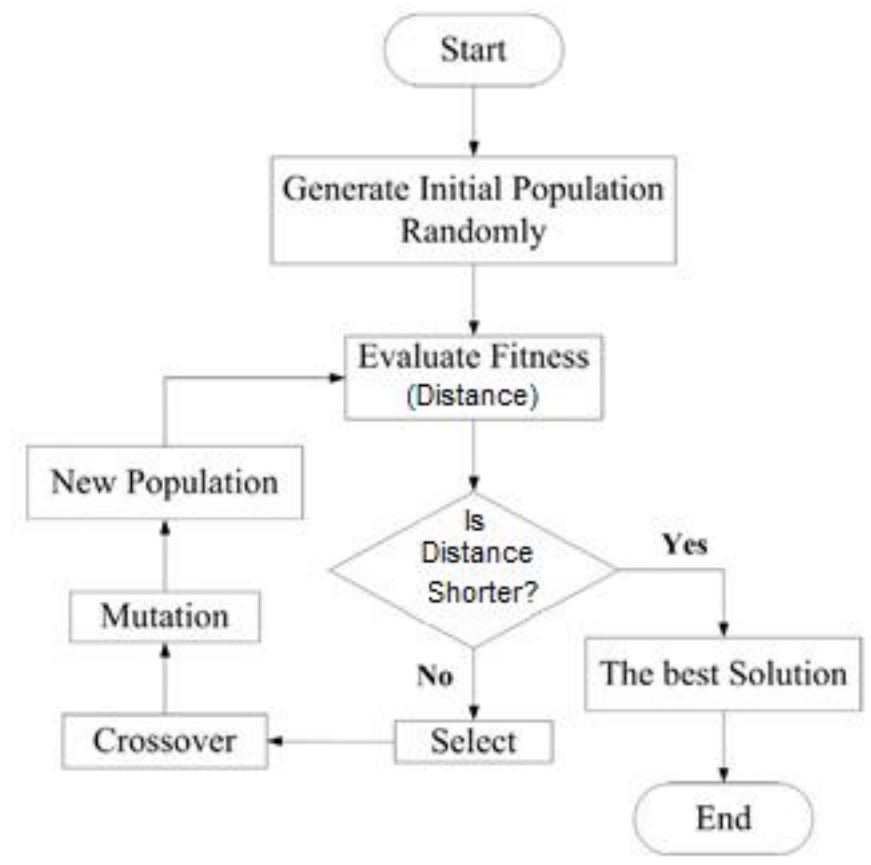

Figure 3.1. The general flow of the Genetic Algorithm used in the benchmarking.

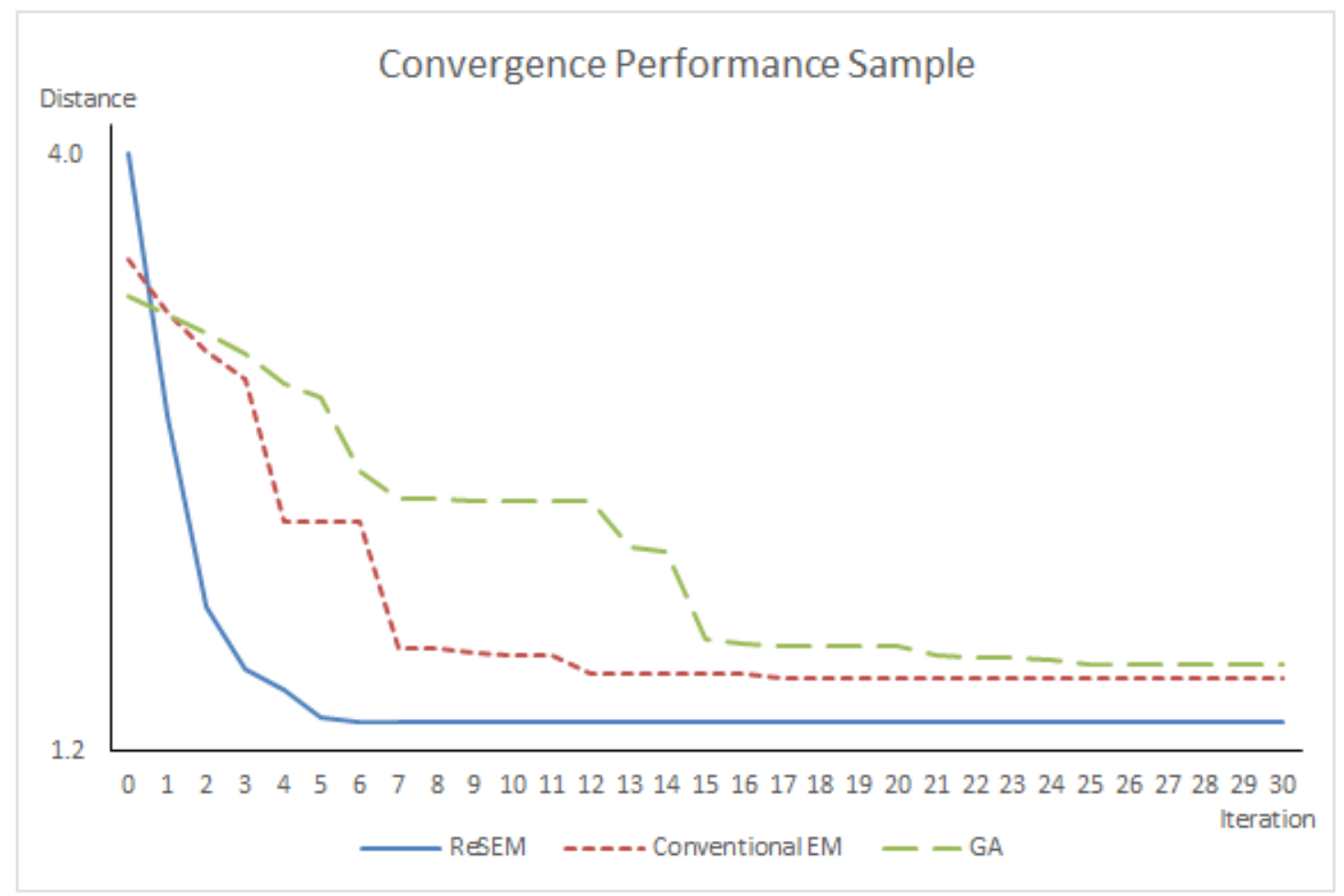

Figure 3.2. Convergence process comparison.

\section{CONCLUSION}

The size of the search steps has been a common issue in the development of optimization algorithms. Larger search steps enable quicker search, but return with less accurate solutions. Scrutinized search, on the other hand, can ensure the algorithm to hit solutions with higher accuracies. The trade-foo, however, is that the search takes relatively longer time. In this research, an enhanced Electromagnetism- 
Like Mechanism algorithm has been developed for the optimization of the tool path of a 3D printing system. A regulated search step mechanism has been introduced into the local search segment of a conventional EM. This regulated search EM (ReSEM) begins the search with a relatively large search step to speed up the convergence process. As the search iterations go, the algorithm then automatically adjust and fine-tunes the search step in order to achieve solutions with higher accuracies. This provides the search algorithm both the advantages in terms of speed and accuracies. Experiments have been carried out to verify and examine the performance of the proposed algorithm. The results indicated that the proposed ReSEM showed significant improvement over the conventional EM and outperformed the GA in the search for the shortest and quickest tool path for the 3D printing system. In the future, this enhanced global optimization search mechanism can also be implemented to solve other engineering optimization and scheduling problems.

\section{REFERENCES}

[1] B. Berman, 3-D printing: the new industrial revolution. Bus. Horiz. 55, 155-162 (2012).

[2] N. Gershenfeld, How to make almost anything-the digital fabrication revolution. Foreign Policy 91 (6), 42-57 (2012).

[3] P. Reeves, Additive Manufacturing-A Supply Chain Wide Response to Economic Uncertainty and Environmental Sustainability. Econolyst Ltd., Derbyshire, UK (2008).

[4] N. Hopkinson, R.J.M. Hague, P.M. Dickens, Rapid Manufacturing. An industrial Revolution for the Digital Age. John Wiley and Sons Ltd., Chischester, West Sussex. UK (2006).

[5] T. Rayna, L. Striukova, Technological Forecasting \& Social Change 102 214-224 (2016).

[6] B. Berman, 3-D printing: the new industrial revolution. Bus. Horiz. 55 (2), 155$162(2012)$.

[7] S.J. Ford, M. Despeisse, Additive manufacturing and sustainability: an exploratory study of the advantages and challenges. J. Clean. Prod. 137, 15731587 (2016).

[8] T. Rayna, L. Striukova, From rapid prototyping to home fabrication: how 3D printing is changing business model innovation. Tech. Forcasting Soc. Chang. 102, 214-224 (2016).

[9] P. Lechowicz, L. Koszalka, I. Pozniak-Koszalka, and A. Kasprzak, Path Optimization in 3D Printer: Algorithms and Experimentation System, 4th International Symposium on Computational and Business Intelligence (2016).

[10] M. Ancău, The optimization of printed circuit board manufacturing by improving the drilling process productivity, Computers \& Industrial Engineering, vol. 55(2), pp. 279-294 (2008).

[11] X. Hong, L. Yuan, Z. Kaifu, Y. Jianfeng, L. Zhenxing, and S. Jianbin, Multiobjective Optimization Method for Automatic Drilling and Riveting Sequence Planning, Chinese Journal of Aeronautics, vol. 23(6), pp. 734-742 (2010).

[12] Z. Q. Li, X. Wang, and Y. F. Dong, ACO-Based Holes Machining Path Optimization Using Helical Milling Operation, Advanced Materials Research, vols. 834-836, pp. 1386-1390 (2014). 
[13] A. T. Abbas, M. F. Aly, and K. Hamza, Optimum drilling path planning for a rectangular matrix of holes using ant colony optimisation, International Journal of Production Research, vol. 49(19), pp. 5877-5891 (2011).

[14] H. Q. Du and J. B. Qi, Application of a Hybrid Algorithm Based on Genetic Algorithm and Hill-Climbing Algorithm to Tool Path Optimization in CNC Machining, Advanced Materials Research, vol. 102(104), pp. 681-685 (2010).

[15] A. Kumar and P. P. Pachauri, Optimization Drilling Sequence by Genetic Algorithm, International Journal of Scientific and Research Publications vol. 2(9), pp. 1-7 (2012).

[16] S.I. Birbil, S.C. Fang, Electromagnetism-like mechanism for global optimization. Journal of Global Optimization 25: 263-282 (2003).

[17] P.T. Wu, Y.Y. Hung, Z.P. Lin Intelligent forecasting system based on integration of electromagnetism-like mechanism and fuzzy neural network. Expert Systems with Applications 41: 2660-2677 (2014). 


\section{ANALYZING SITE SUITABILITY FOR SOLID WASTE DISPOSAL THROUGH GIS MULTI-CRITERIA DECISION MAKING HIERARCHY PROCESS}

Sikandar Ali Shah

Department of Geography, University of Karachi, (Pakistan)

E-mail: sayed.sikandar24@gmail.com

Prof. Dr. Engr. Sayed Hyder Abbas Musvi

Indus University, Karachi, (Pakistan)

E-mail: dean@indus.edu.pk

Abeela Tameez

Department of Geography, University of Karachi, (Pakistan)

Masood Alam

Department of Geography, University of Karachi, (Pakistan)

Asim Nawaz

Department of Geography, University of Karachi, (Pakistan) 


\section{ABSTRACT}

Currently the human beings are encircled by multiple types of problems, out of those the environmental degradation issue is one of the major problem which is caused by over population, rapid increase of urbanization and industrialization which result in production of huge quantity of solid wastes. Thus the effectual solid waste management and its disposal arrangements are required. Another major problem being faced globally is to locate an appropriate area for the disposal of hazardous wastes by overcoming main constraints including environmental, economic and political for spotting of a suitable site. In this study our focus is to spot a proper landfill site for the disposal of hazardous wastes for which we have selected industrial (radioactive) wastes of the district of Karachi East as a area for research. This district has wide range of Korangi Industrial Area and small portion of Landhi Industrial Area. In this research we have used methods of Decision Support System (DSS) based on Geographical Information System (GIS) and Remote Sensing (RS) concerning all supportable measures. With the assist of Analytic Hierarchy Process (AHP) and Multi-Criteria Decision Making (MCDM), suitable alternative site for dumping solid waste has been selected on the basis of different factors performed on Arc Map 10.3, Erdas Imagine, e-Cognition developer by considering the decision maker's expectations. The results show that after performing different investigations on the suitability indexes, the analyzed area which is most suitable for solid waste dumping is $200 \mathrm{~m}$ away from Malir River.

\section{KEYWORDS}

Decision support system (DSS), landfill site, hazardous wastes, Multi-Criteria Decision Making (MCDM), Spatial Decision Support System (SDSS), Analysis Hierarchy Process (AHP).

\section{INTRODUCTION}

Solid Waste (SW) material contains all kinds of surplus such as trash, waste, sludge and few more solid garbage that are produced from human and other living thing's activities which is usually rejected to reuse or recycle including industrial, agricultural, commercial and society activities excluding sewage material from residential area, silt in water, poised solids in wastewater sewage from industries, suspended particles in irrigation system and other multiple common types of water pollutants. From the Document Driven Decision Support System's perspective which is applied in this study, Solid Waste Management (SWM) is one of the most important services which are getting extensive attention in the urban agenda of several developing countries [1]. Due to less concentration of SWM, it has been observed that there are numerous environmental degradation vulnerabilities along with health risks. This problem becomes wider and reaches beyond the geographical margins of towns or cities. Generally the Solid waste management is a calamity in several major urban regions of the world, as populations are attracted toward cities which continue to increase urbanization leading to continuously increasing amount of residential solid waste; whereas space for disposal is limited. Similarly in many developing cities and towns, inapt managing and disposal of municipal solid waste is most conspicuous reason of environmental degradation [2], for example air, water, soil and land pollution etc, which is caused by inappropriate disposal of municipal solid wastes. Although in the early 1990s; about US $\$ 25$ billion is spent annually just on solid waste management by Asian countries this figure is estimated 
to increase approximately US $\$ 50$ billion by 2025[3]. Rapid Increase in population results in rising quantity of solid waste with higher rate and has remained a challenge for Pakistan also, however it has been examined that the spawned waste is carried out and either dumped in low lying areas or burned in open environment exclusive of analyzing the disposal hazards and spot. This study has been carried out to assess the possibility of landfill site selection for solid waste dumping (SWD). Presently, Pakistan is suffering from solid waste issue and has received lesser attention for an adequate and appropriate ways of collection, transportation, disposal and dumping due to the large sizes of its cities, consequently, the environmental and sanitary conditions are becoming critical with the passage of time, and thus public social life is continuously deteriorating [4]. According to survey report of 1998 out of 130.579 million population of Pakistan, 67\% is living in countryside areas, whereas $33 \%$ reside in cities. Moreover, out of $33 \%$ of people existing in cities, from which $54 \%$ exist in 10 main cities of Pakistan [5]. Throughout the past various decades, migration has begun from rural to urban regions. The main reasons which cause this migration are as less crop yields, slow growth in agricultural zones, unemployment and environmental damages because of deforestation, water scarcity and desertification. The huge countryside inundation has, sequentially added to the overload on urban infrastructure and services [6]. The at present approximately 6000 tons per day waste dumping is required in Karachi which is one of the largest city of Sindh province of Pakistan, but it has not been conducted in adequate and appropriate manners in collection, transportation and disposal, as a result, the sanitary and environmental circumstances have further worsened, as well as public is suffering from passing their lives in polluted situation. These exposure consist on expending rate of urban areas, structure and compactness of urban regions, planning and reign of progress, temperature and precipitation, scavenger's commotion for recyclable partition, ability sufficiency as well as the restrictions of several precincts to control the waste damage, collect, transport and dispose [7]. Karachi is a metropolitan city of Pakistan has inappropriate municipal solid waste management system as well. The existing solid waste collection and transportation management system in Karachi is not perfect. There is inadequacy for extensive term plans, consequently in some cases rambling, ad-hoc and often damaging plan, guidelines and organizational schemes exist. The municipal infrastructure construction has been lagged by the economic development, which become the bottle-neck to hinder the faster and better development of the municipal economy. Waste collection, disposal, transport and street sweeping, duty lies with the municipal authorities. It has created suggestion for improved container design and promotes consciousness of solid waste management [8].

\section{STUDY AREA}

Karachi East is a densely populated district of Karachi city and it has a large important and famous areas such as Jinnah International Airport, PAF Base Faisal, PAF Museum, Pakistan Maritime Museum, Karachi Universty, NED University, Sir Syed University of Engineering and Technology, Federal Urdu University Of Arts Science \& Technology, Bahria University, Safari Park, Aladin Amusement Park, Sindhbad Play Land, Liaquat National Hospital, Agha Khan Hospital, Korangi Industrial Area and some part of Landhi Industrial Area; all covering an area of 
$140 \mathrm{~km} 2$. It is located at $24^{\circ} 45^{\prime \prime}$ to $25^{\circ} 15^{\prime \prime}$ north and $66^{\circ} 37^{\prime}$ to $67^{\circ} 37^{\prime \prime}$ east. It has Malir District in the north-east, Karachi West District in the North-West, Karachi South District in the south and Karachi Central District to the west as shown in Fig.1 and located near to the coast resulting relatively mild climate.

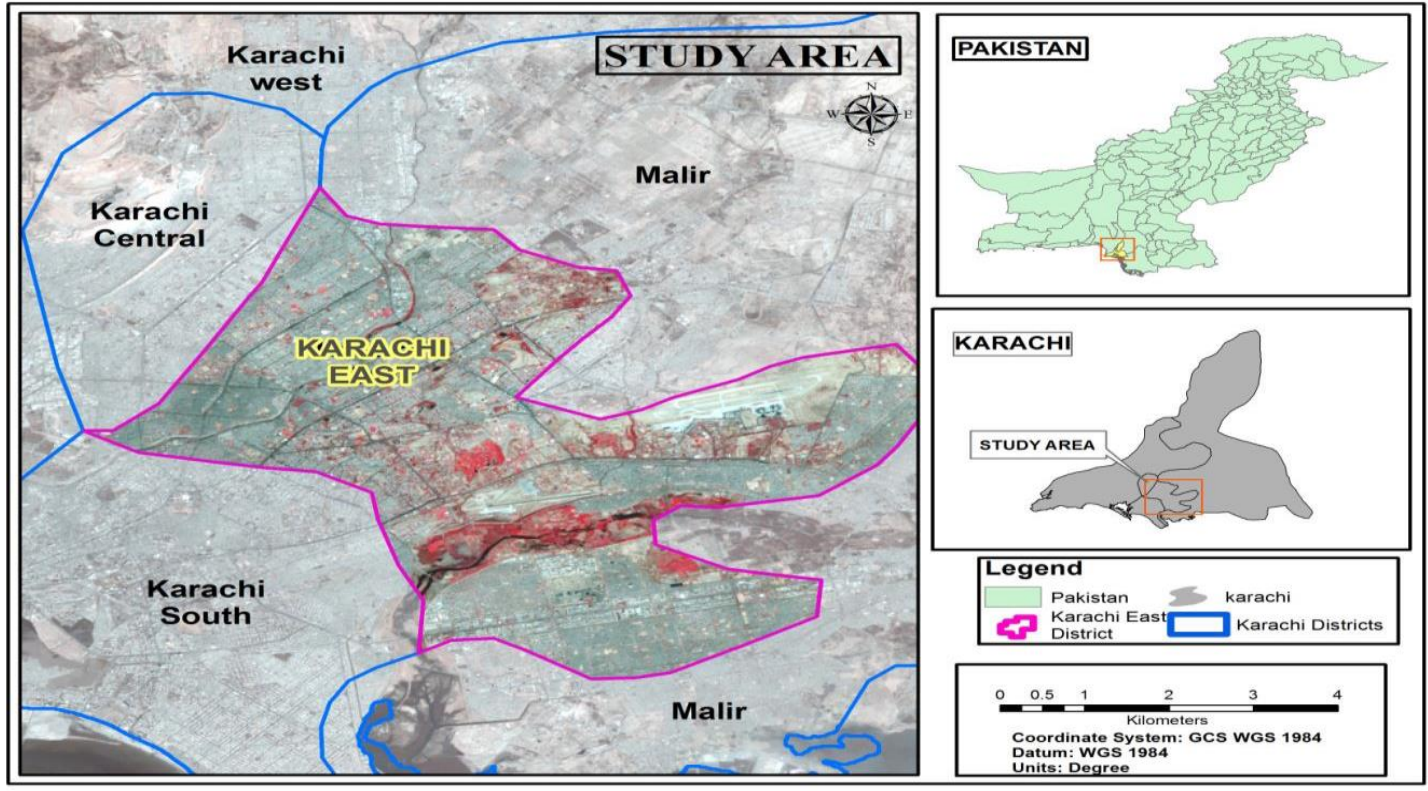

Figure 1. Location of Karachi East Pakistan.

The district of Karachi East has been shifted through knowledge driven Decision Support System which is analyzed by (SWOT) (planning method/tool) shown in Figure 2 and its outreach described in suitability analysis [9]. Therefore, our purpose is to use the technique of SWOT planning expeditious in life cycle of OBIA

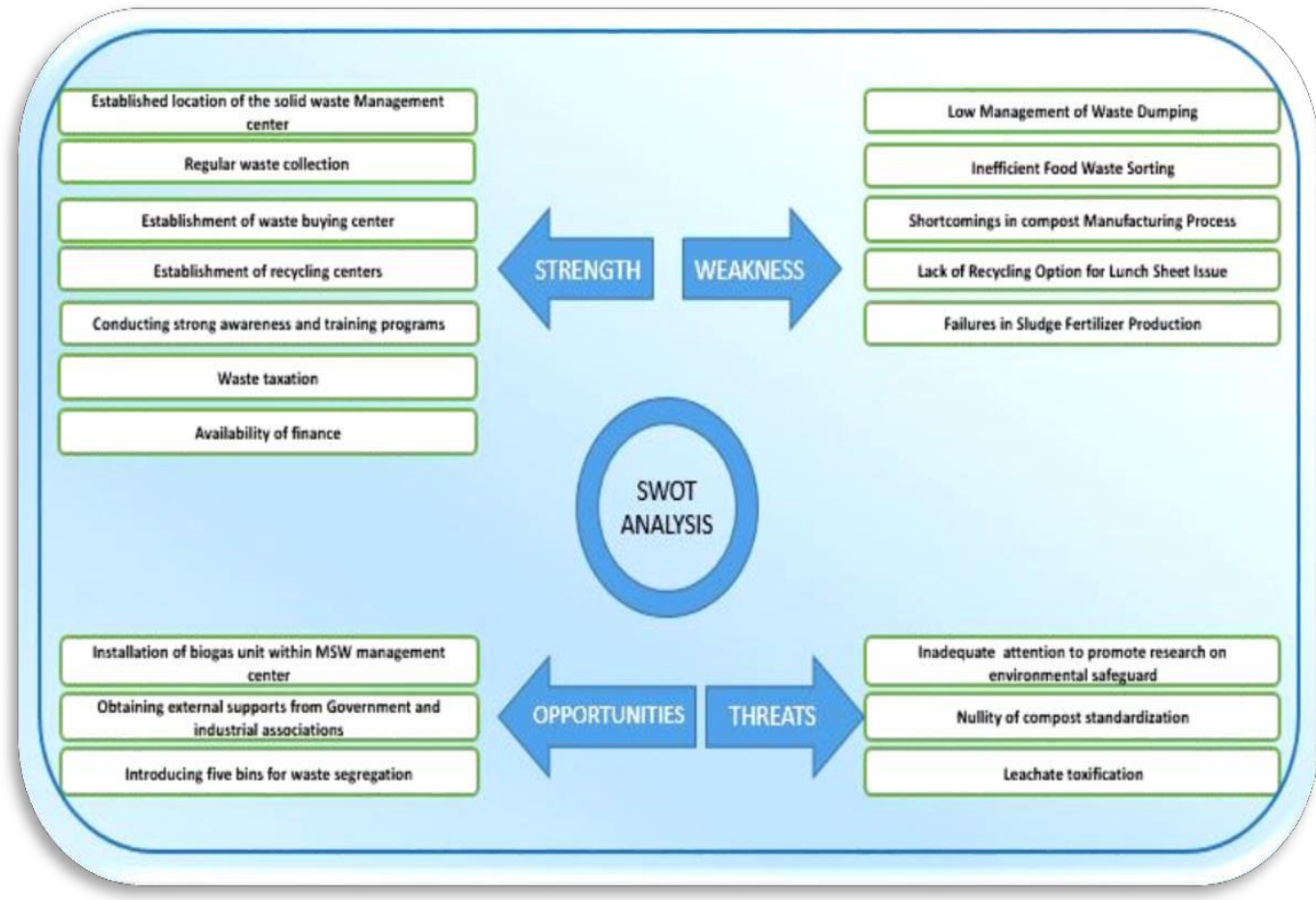

Figure 2. Flow Diagram is describing strength, weakness, opportunity and threats of solid waste dumping in the study area. 
by means of the intention whose notions explained at this time can be used to reinforce and direct this rising discipline[10].

\section{METHODOLOGY}

The process of Analytic hierarchy data driven decision support system is classified in Figure 3 to Figure 5 with the help of Simon's Model, where data has been gathered, processed and analyzed for the suitability indexes and after that finalized through efficiently methods of RS and GIS.

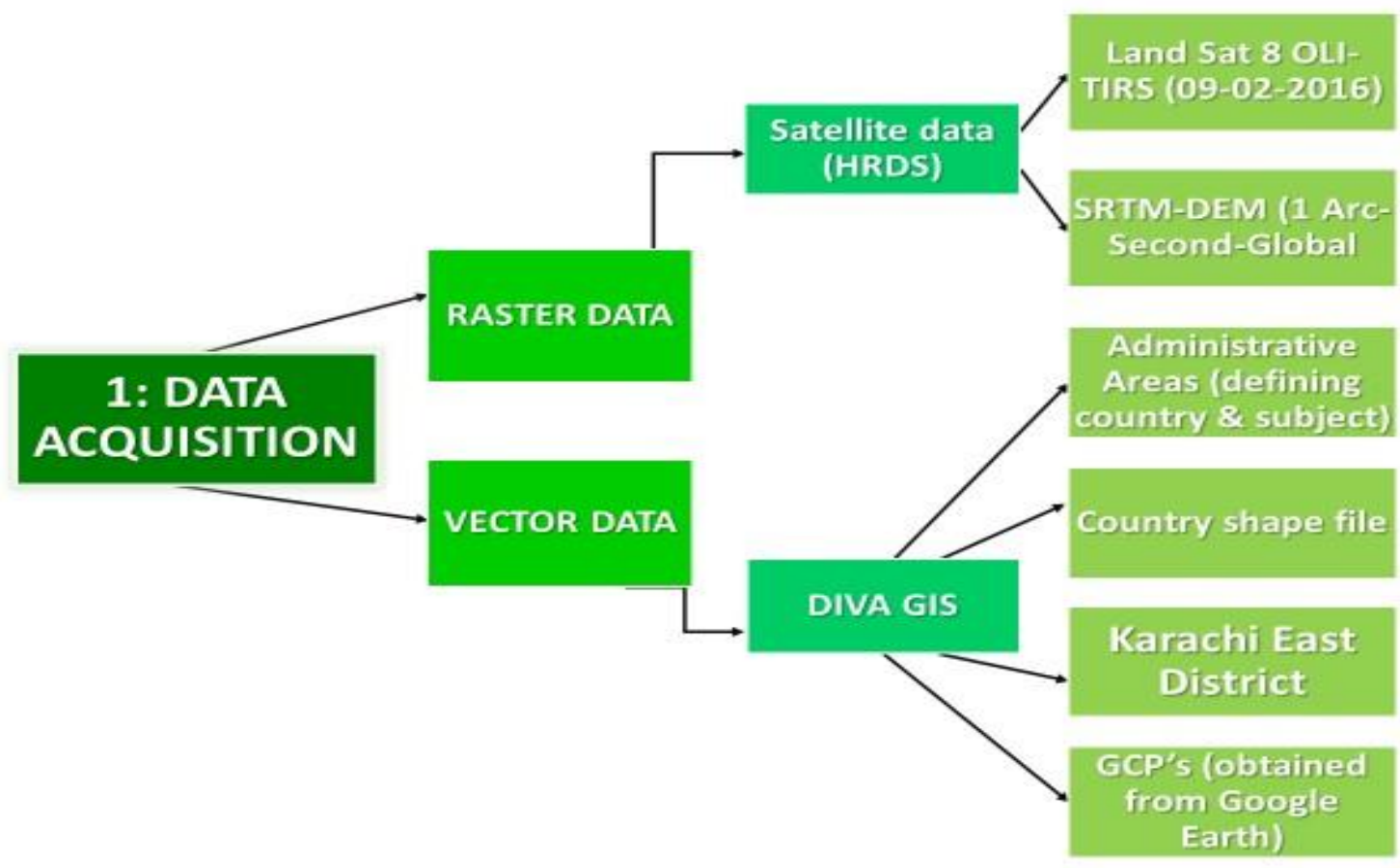

Figure 3. Flow Chart showing data acquisition.

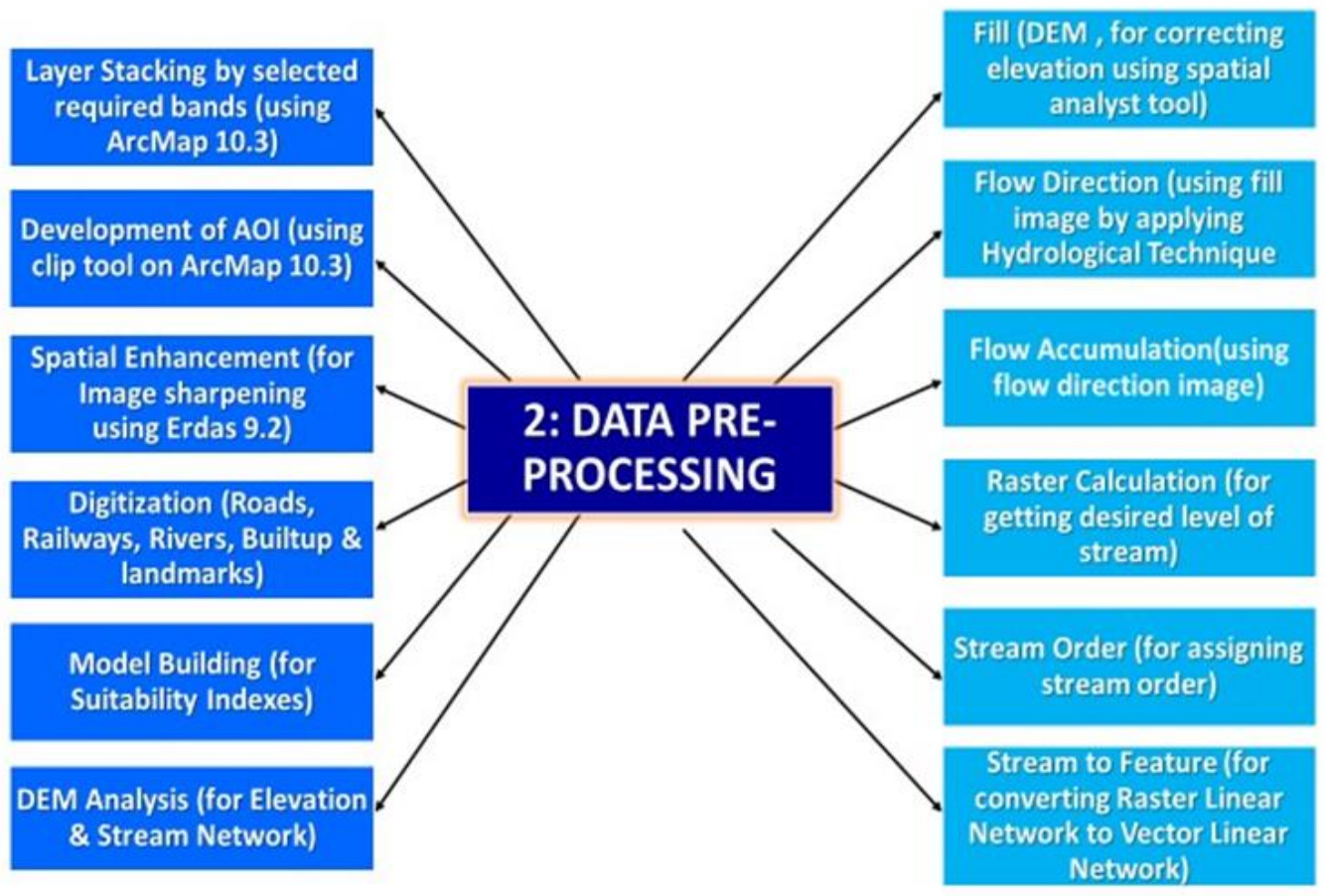

Figure 4. Flow Chart showing data pre - processing. 


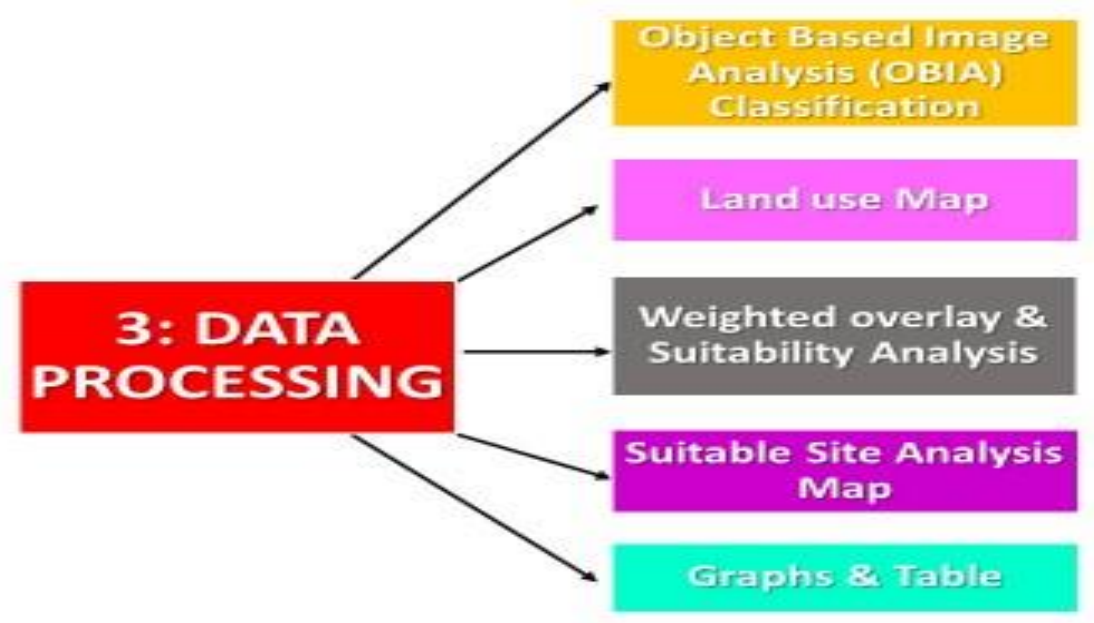

Figure 5. Flow Chart showing data processing.

\section{RESULTS AND DISCUSSIONS}

Communication driven decision support system used to choose the study area that improved decision making capabilities throughout the work done. Similarly, model driven support system manipulated the data to produce analytical results. Although Karachi East District does not have uneven (hilly) surface which contains low elevation level therefore few water bodies have came out in results given in Figure 6. Simultaneously, area is totally filled with land use features which have resulted in Figure 7 .

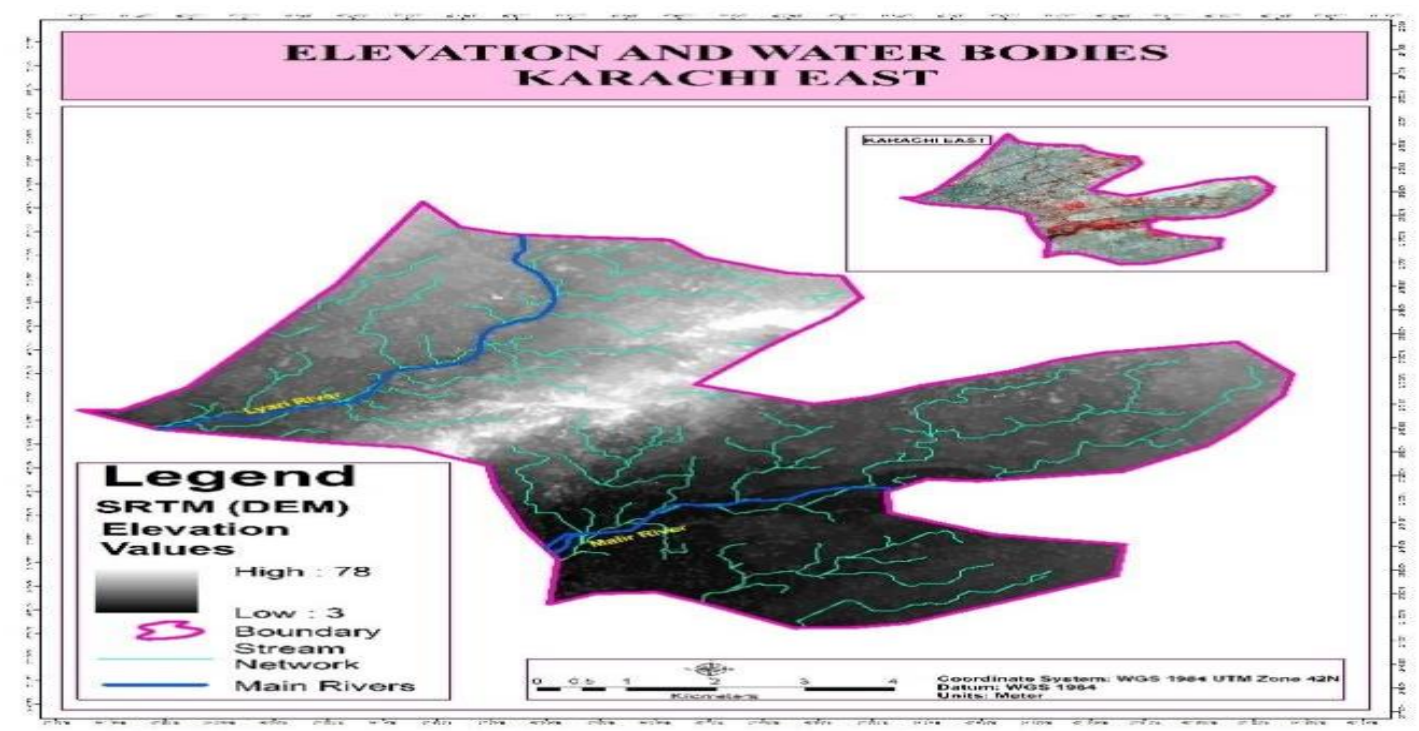

Figure 6. Elevation and water bodies lie in the Karachi East. 


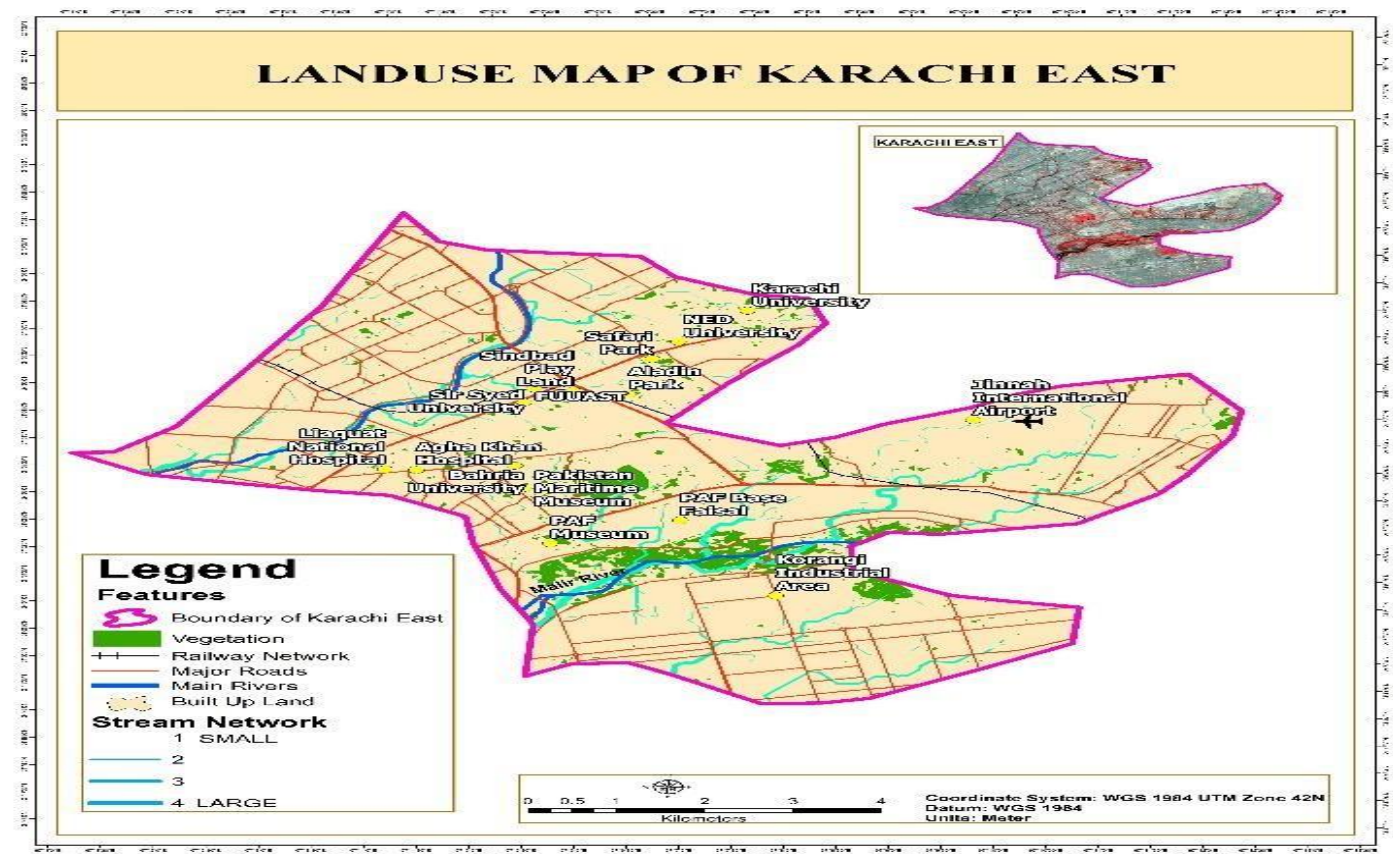

Figure 7. Land Use Map of Study Area.

Karachi city has 18 towns with 11 Dumping sites as shown in Table 1. But the identified dumping sites on the basis of origin are Jam Chakro, Gond Pass, Saba Cinema (North Karachi), Korangi 5, Lalabad (Landhi), Korangi 3.5 (Ibrahim Haidry Police Station) and Korangi 1.5 by 100 quarters. While main wastage dump in Gond Pass (1600 tons/day) and Jam Chakro (1200 tons/day) that means it represent approximately $45 \%$ of the total and there is only one dumping site which is not located on its best location for the whole district as shown in Figure 8. Moreover, the Sindh Solid Waste Management Board (SSWMB) has taken over the land of Karachi Development Authority (KDA) adjacent to Race Course near Safoora Chowrangi for the construction of a Garbage Transfer Station (GTS). However, building the GTS in District East is likely to prove hazardous for the city [11]. Additionally, researches describe that more landfill sites should be placed in urban region but far from populated areas rather than rural areas due to bulky amount of solid waste found in urban sites.

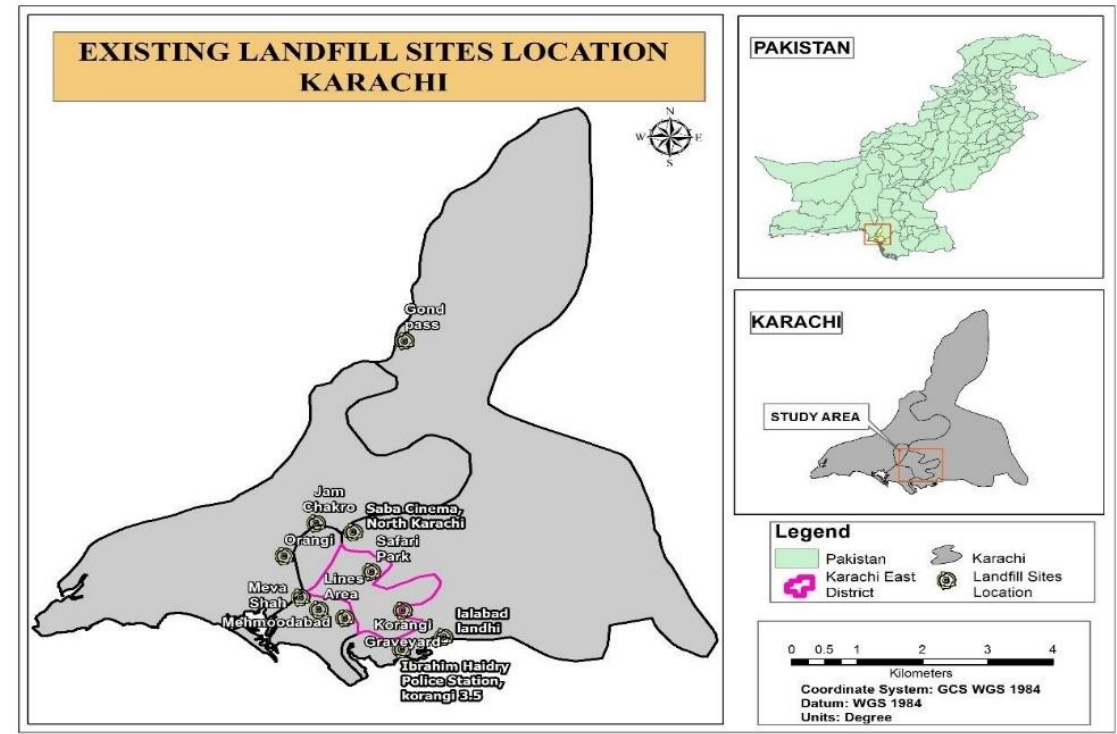

Figure 8. Map showing location of existing landfill sites in Karachi. 
Table 1. Locations of Landfill Sites in Karachi.

\begin{tabular}{|r|l|l|l|}
\hline S.No & \multicolumn{1}{|c|}{ Name of Site } & \multicolumn{2}{|c|}{ Coordinates } \\
\hline 1 & Jam Chakro & $\mathrm{N}=25^{\circ} 01.675^{\prime}$ & $\mathrm{E}=67^{\circ} 01.61^{\prime}$ \\
\hline 2 & Deh Gond Pass & $\mathrm{N}=25^{\circ} 00.634^{\prime}$ & $\mathrm{E}=66^{\circ} 55.263^{\prime}$ \\
\hline 3 & Mehmoodabad & $\mathrm{N}=24^{\circ} 50.906^{\prime}$ & $\mathrm{E}=67^{\circ} 04.212^{\prime}$ \\
\hline 4 & Safari Park & $\mathrm{N}=24^{\circ} 55.241^{\prime}$ & $\mathrm{E}=67^{\circ} 06.391^{\prime}$ \\
\hline 5 & Lines Area & $\mathrm{N}=24^{\circ} 51.903^{\prime}$ & $\mathrm{E}=67^{\circ} 02.292^{\prime}$ \\
\hline 6 & Orangi & $\mathrm{N}=24^{\circ} 56.210^{\prime}$ & $\mathrm{E}=67^{\circ} 00.379^{\prime}$ \\
\hline 7 & Meva Shah & $\mathrm{N}=24^{\circ} 53.332^{\prime}$ & $\mathrm{E}=67^{\circ} 00.338^{\prime}$ \\
\hline 8 & Korangi Graveyard & $\mathrm{N}=24^{\circ} 51.527^{\prime}$ & $\mathrm{E}=67^{\circ} 11.206^{\prime}$ \\
\hline 9 & Saba Cinema, North Karachi & $\mathrm{N}=24^{\circ} 59.250^{\prime}$ & $\mathrm{E}=67^{\circ} 5.169^{\prime}$ \\
\hline 10 & lalabad landhi & $\mathrm{N}=24^{\circ} 49.385^{\prime}$ & $\mathrm{E}=67^{\circ} 12.485^{\prime}$ \\
\hline 11 & Ibrahim Haidry Police Station & $\mathrm{N}=24^{\circ} 48.238^{\prime}$ & $\mathrm{E}=67^{\circ} 9.105^{\prime}$ \\
\hline
\end{tabular}

For getting better multi criteria decision making results which reduces outlays and speed turn-around on the next land-use/land-cover, vegetation or impermeable surface mapping project, Object-Based Image Analysis (OBIA) was performed initially on E-cognition Developer which is advanced method of image classification shown in Figure 9. Multi-scale segmentation has frequently been associated with theory of hierarchy [12].

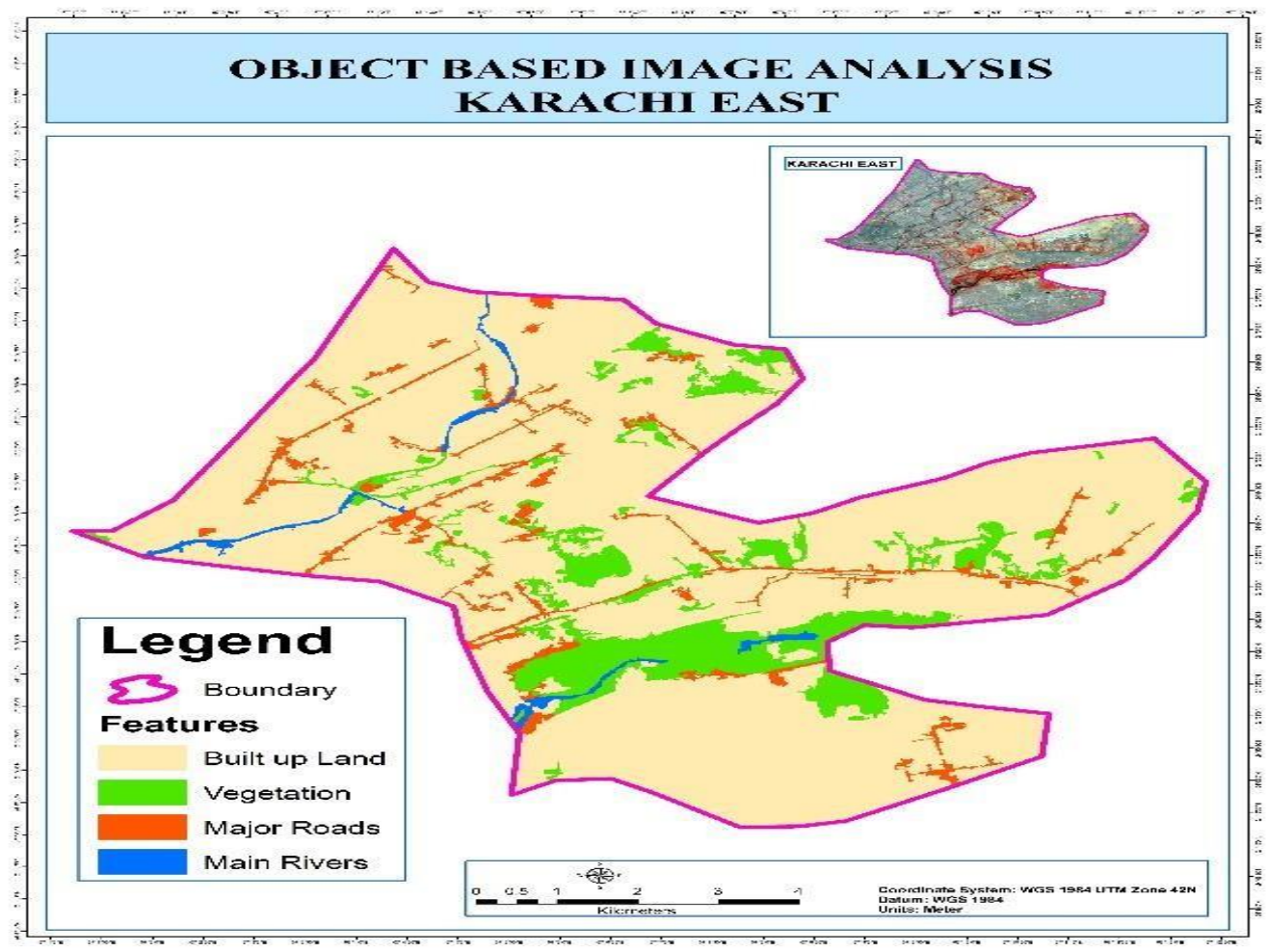

Figure 9. Map showing OBIA classification.

Figure 9 depicts the OBIA classification besides above technique, weighted overlay and suitability analysis is carried out on ArcGIS 10.3 software with the help of distinct aspects and creation of models in model builder used for selecting suitable land disposal site (Model: I, II \& III) as shown in fig. 10, 11 and 12. The problem over here is to make a suitability raster for the area of solid waste dumping site. These equations $1,2 \& 3$ define the suitability to be the total of weight criteria multiplied with the product of restrictions. 
$S=\sum_{i=1}^{n} w_{i} C_{i} \prod_{j=1}^{m} r_{j^{----------~} \rightarrow 1}$

Where

$-\mathrm{S}=$ Suitability for a waste disposal site

$-W_{i}=$ Weight for Criteria $i C_{i}$

$-C_{i}=$ Criteria for suitability

$-r i=$ Restriction

\section{Restriction Model \\ $S=\sum_{i=1}^{n} w_{i} C_{i} \prod_{j=1}^{m} r_{j}$

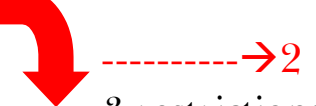 \\ 3 restrictions}

$S=\sum_{i=1}^{n} \mathrm{w}_{\mathrm{i}} \mathrm{C}_{\mathrm{i}}\left(\mathrm{r}_{\text {rivers }} * \mathrm{r}_{\text {roads }} * \mathrm{r}_{\text {landmarks }}\right)------->3$

Where,

$-\mathbf{r}_{\text {rivers }}=$ Restriction related to river location

$-\mathbf{r}_{\text {roads }}=$ Restriction related to roads location

$-\mathbf{r}_{\text {landmarks }}=$ Restriction related to parks and reserves location

Restriction in Buffer is shown in Table 2. Most restrictions included a Minimum and Maximum Buffer Zone with Analysis Buffer.

Table 2. Restriction Buffer.

\begin{tabular}{|l|c|c|c|}
\hline Restriction Source & $\begin{array}{c}\text { Minimum Buffer } \\
\text { Distance (m) }\end{array}$ & $\begin{array}{c}\text { Maximum } \\
\text { Buffer Distance } \\
(\mathbf{m})\end{array}$ & $\begin{array}{c}\text { Analysis Buffer } \\
\text { Distance (m) }\end{array}$ \\
\hline Rivers & 30 & 200 & 200 \\
\hline Roads & 50 & 300 & 300 \\
\hline Landmarks & 300 & 3000 & 1000 \\
\hline
\end{tabular}

Restriction in Raster are shown in Table 3.

Restrictions in raster exposed here through Boolean expression where restricted cell is shown with 0 and viable cell is represented with 1 to develop a waste disposal site.

Table 3. Restrictions in Raster.

\begin{tabular}{|c|c|c|c|}
\hline 0 & 1 & 1 & 0 \\
\hline 1 & 1 & 0 & 0 \\
\hline 1 & 1 & 1 & 0 \\
\hline 1 & 0 & 0 & 0 \\
\hline
\end{tabular}




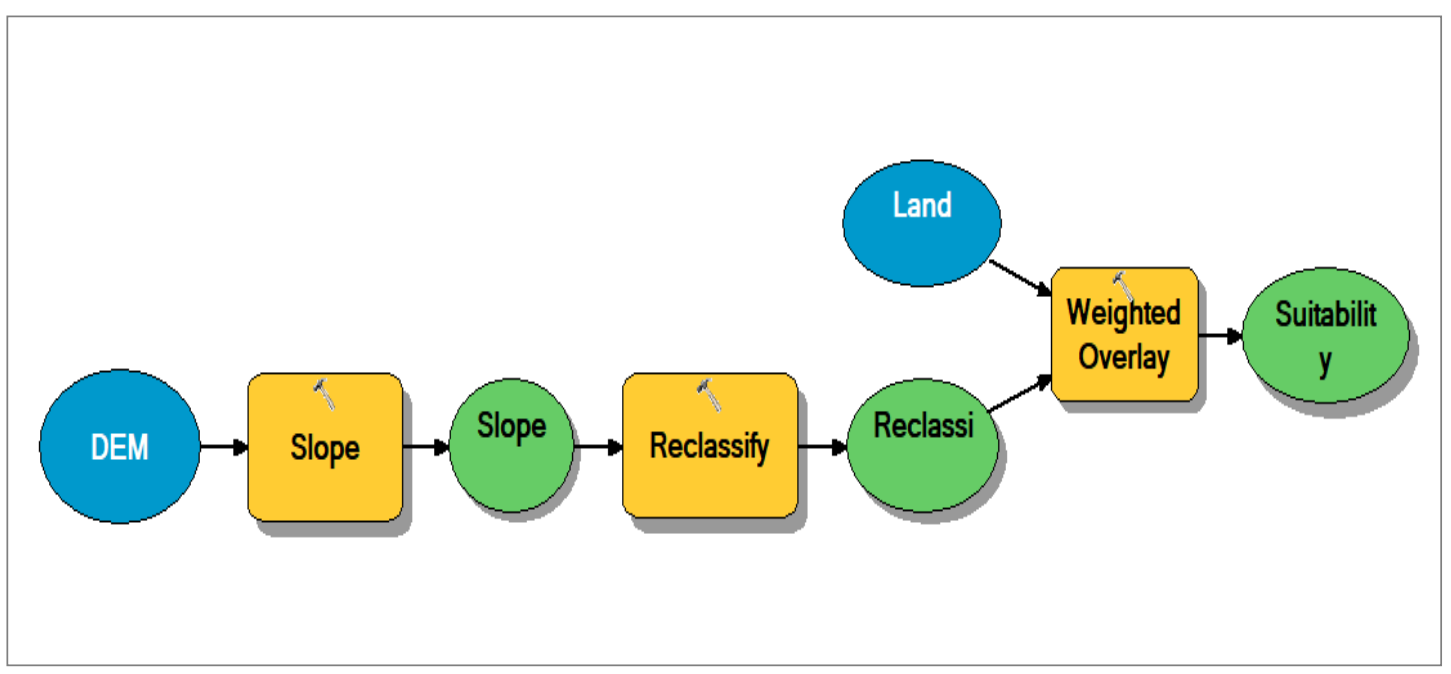

Figure 10. Model I Suitability Model.

$S=\sum_{i=1}^{n} w_{i} C_{i} \prod_{j=1}^{m} r_{j}$

$S=\sum_{i=0}^{n}\left(w_{s} C_{s} * w_{l u} C_{l u}\right) \prod_{j=1}^{m} r_{j}$

Where:

$-w_{s} \& C_{s}=$ Weight and criteria for slope

$-w_{l u} \& C_{l u}=$ Weight and criteria for land use

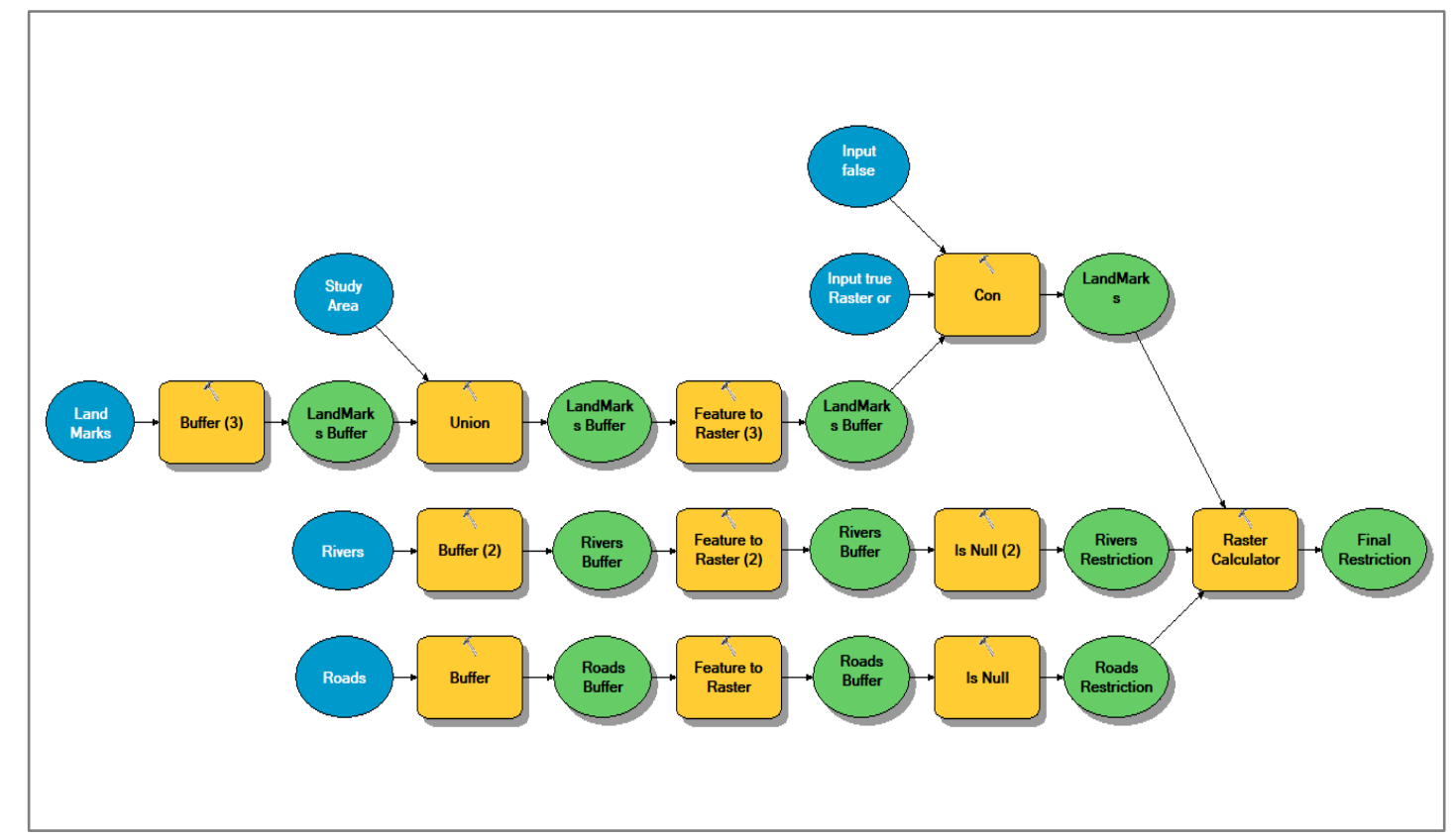

Figure 11. Model II: Weights and Evaluation Scale.

\section{Weights and Evaluation Scale}

- Weights

$\mathrm{S}=\sum_{i=1}^{n}\left(0.40 C_{S} * 0.60 C_{l u}\right) \prod_{j=1}^{m} r_{j}$

- Evaluation Scale

---1 ---5 step of 1 


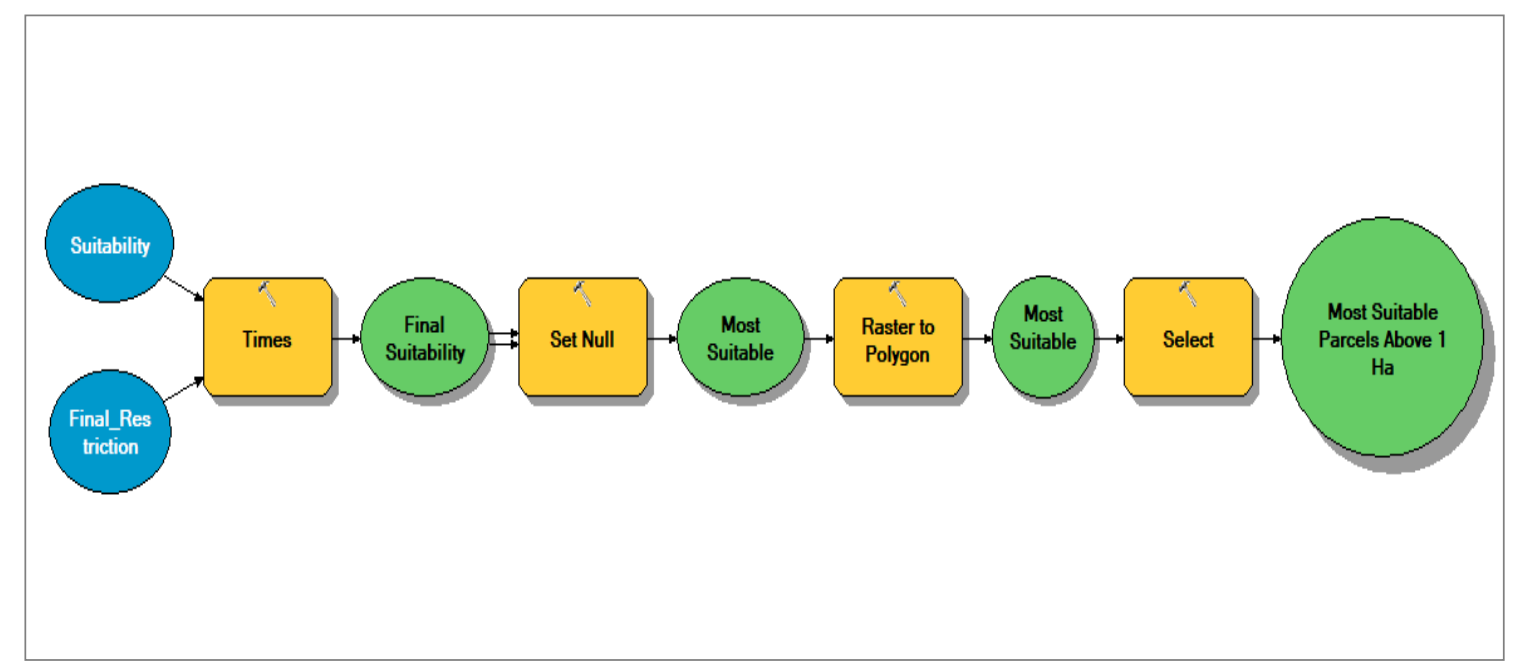

Figure 12. Model III: Parcel Selection Criteria 1.

For selecting best suitability value i.e. 3 in this analysis, parcel selection criteria have been applied whereas most suitable parcels are above than one hector $(10,000 \mathrm{~m} 2)$ in Karachi East's boundary (Model: III).

\subsection{Landmarks Buffer to Landmarks Raster Buffer with Restriction constants}

District East Karachi consists of many famous landmarks shown in Figure 13 (A). Solid waste dumping site should not be the surroundings of landmarks. For this purpose, a $1000 \mathrm{~m}$ buffer zone is applied from all landmarks. Landmarks buffer have been united with the boundary of study area for finding the extent in Figure 13 (B). Whereas Restriction constants -1 (high restriction) and 1 (low restriction) is applied in Raster Buffer zone to determine unacceptable areas for solid waste dumping in Figure $13(\mathrm{C})$.
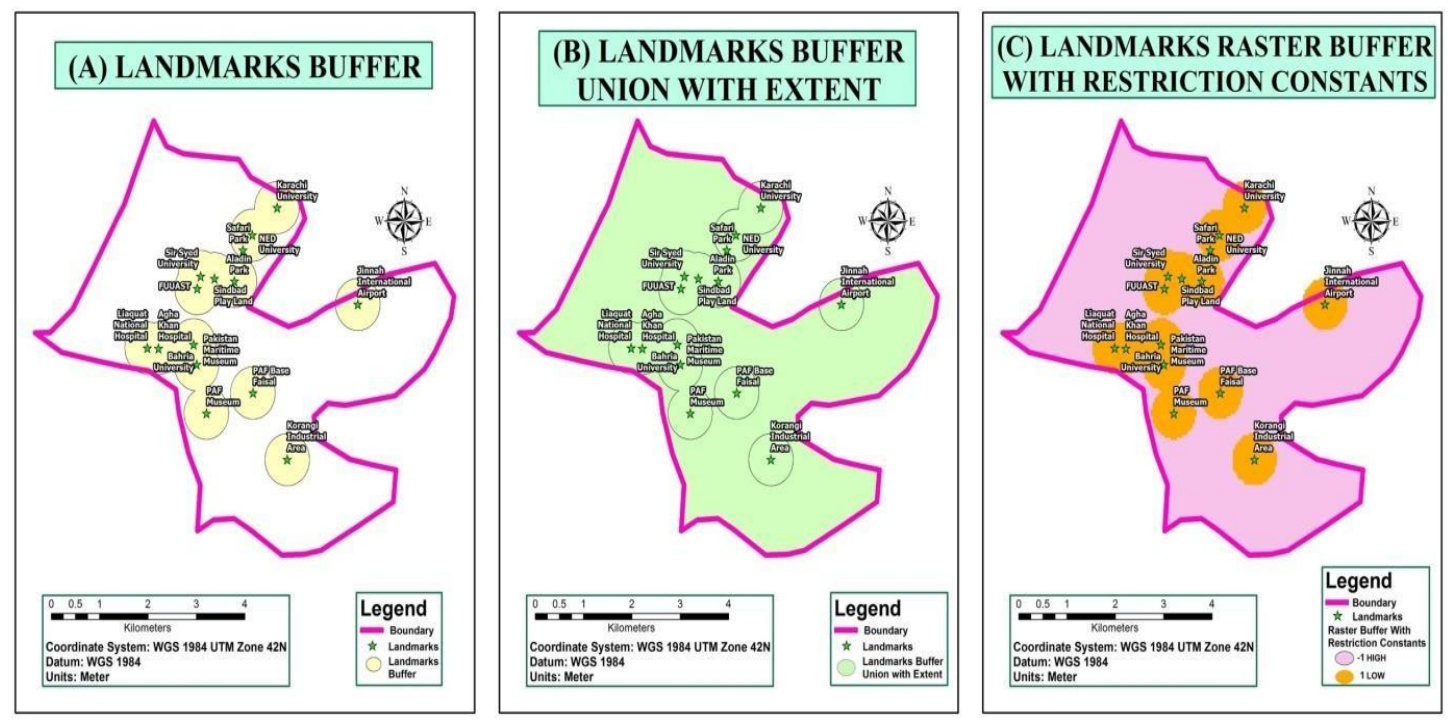

Figure 13. Maps of Suitability Indexes: (A) Landmarks Buffer (B) Landmarks Buffer union with extent (C) Landmarks Raster Buffer with Restriction Constants.

\subsection{Rivers B uffer to River Restriction}

The waste dumping areas should be away from rivers, lakes, or swamps where the underground water level is high. As the main rivers have an excessive discharge and immense downstream impact, therefore no landfill should be placed in the 
floodplains of main rivers [13]. Consequently, a buffer of $200 \mathrm{~m}$ is applied in Figure 14 (D). For getting desire river restrictions output a layer of vector river buffer converted into raster river buffer in Figure 14 (E) because Boolean Expression can be applied on Raster Calculator using raster layer. Result of river restriction is shown in Figure 14 (F) with the value of 0 (high restriction) and 1 (low restriction).
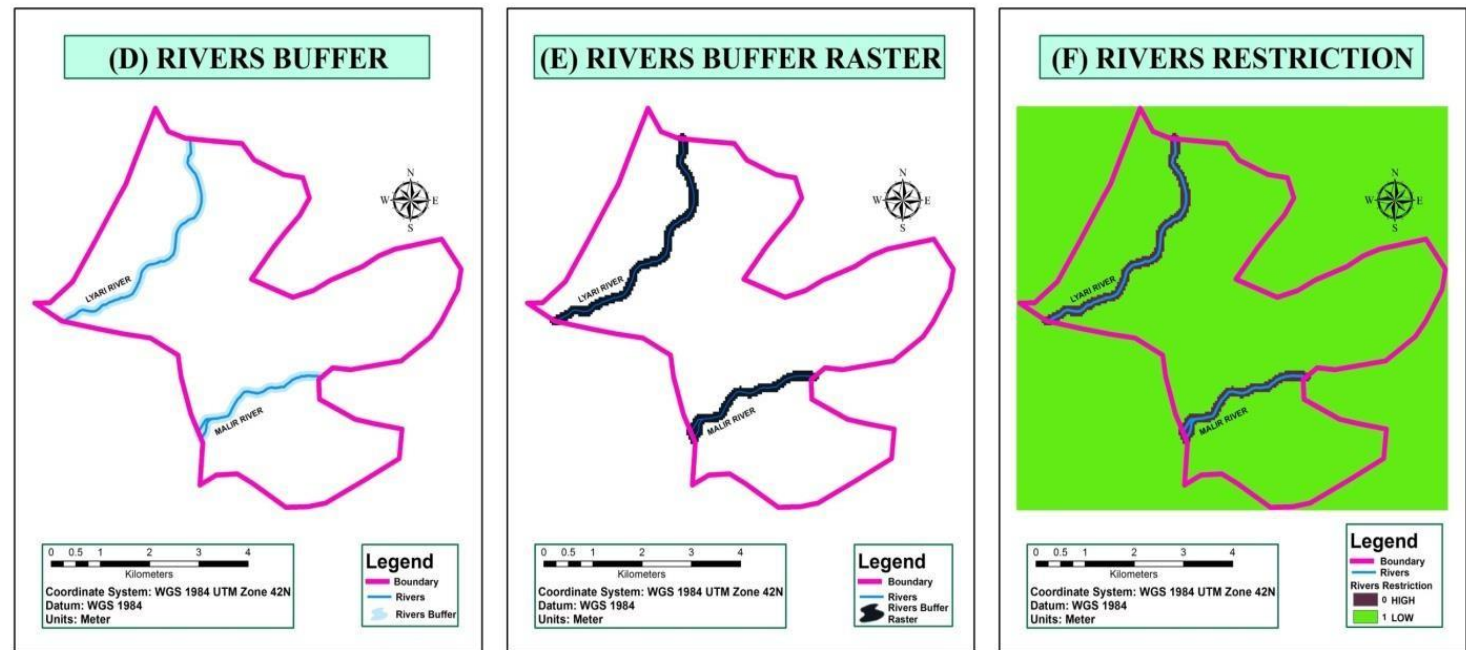

Figure 14. Maps of Suitability Indexes: (D) River Buffer (E) River Buffer Raster (F) River Restriction.

\subsection{Roads Distance to Final Raster Restrictions constants}

The District consists of main roads, secondary roads and pedestrian roads. The waste dumping sites should not be near to the road networks. Therefore, a $300 \mathrm{~m}$ buffer zone is applied to these networks shown in Figure 15(G) Raster Buffer is showing restriction constant where Boolean expression stated high restriction 0 and low restriction 1 for solid waste land disposal in Figure 15(H) Output of Final Raster restriction constants with rivers, roads and Landmarks also gave result with Boolean expression such as 0 (high restriction for solid waste dumping) and 1 (low restriction for solid waste dumping) in Figure 15(I).
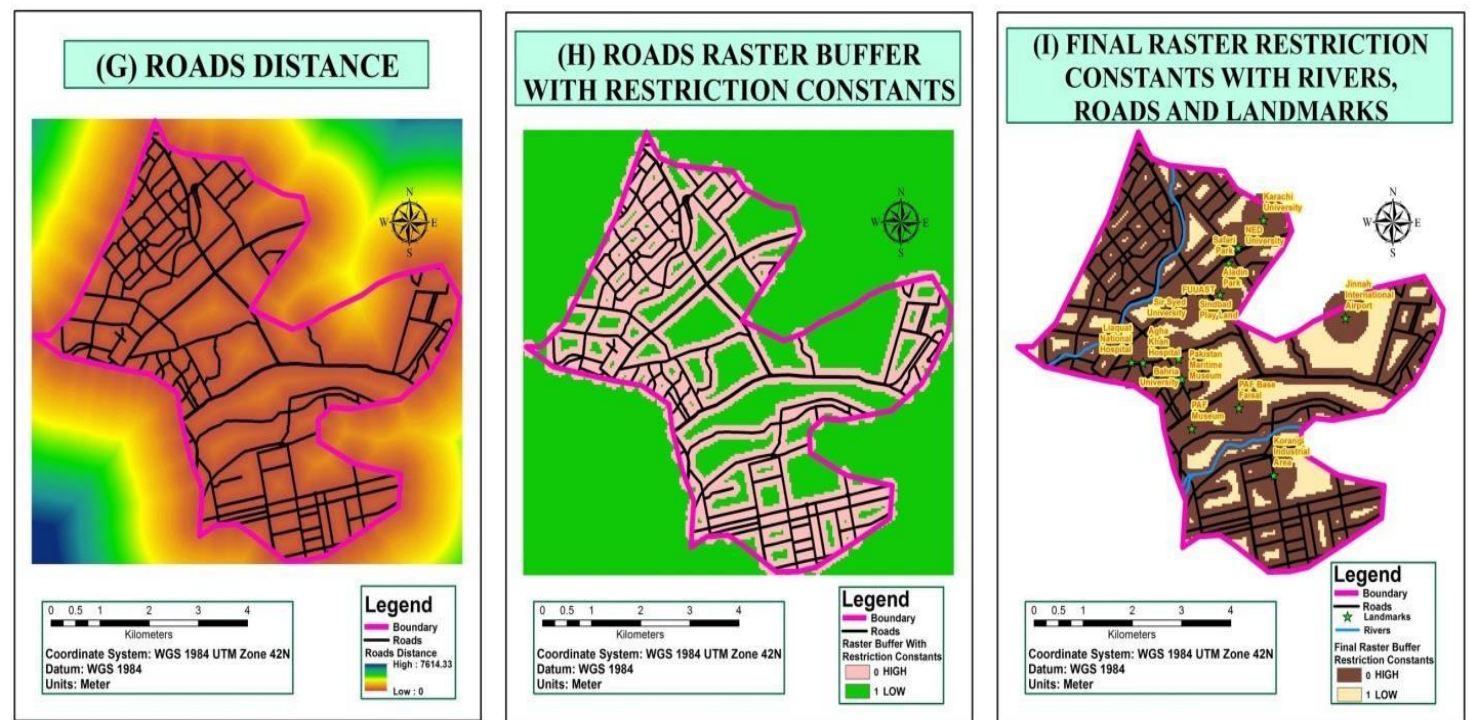

Figure 15. Maps of Suitability Indexes: $(\mathrm{G})$ Roads Distance $(\mathrm{H})$ Roads Raster Buffer with Restriction Constants (I) Final Restriction Constants with Rivers, Roads and Landmarks. 


\subsection{Suitability Ratio to Most Suitable Parcel}

Most of the land disposal site analyzed at a constant given distance from rivers and roads and far from populated areas shown in Fig 16(J). Thus, additional evaluation was done in ArcGIS environment to get most suitable parcels at high suitable ratio in Fig 16(K). Finally, the suitability indexes excluded small parcels that were less than 1 hectare and highlighted the areas in circle (red) from the sites classified as high suitable for

solid waste dumping whereas with black box as shown in Fig 16(L) is most highly appropriate spot for solid waste dumping which is $200 \mathrm{~m}$ away from Malir River in the study area.
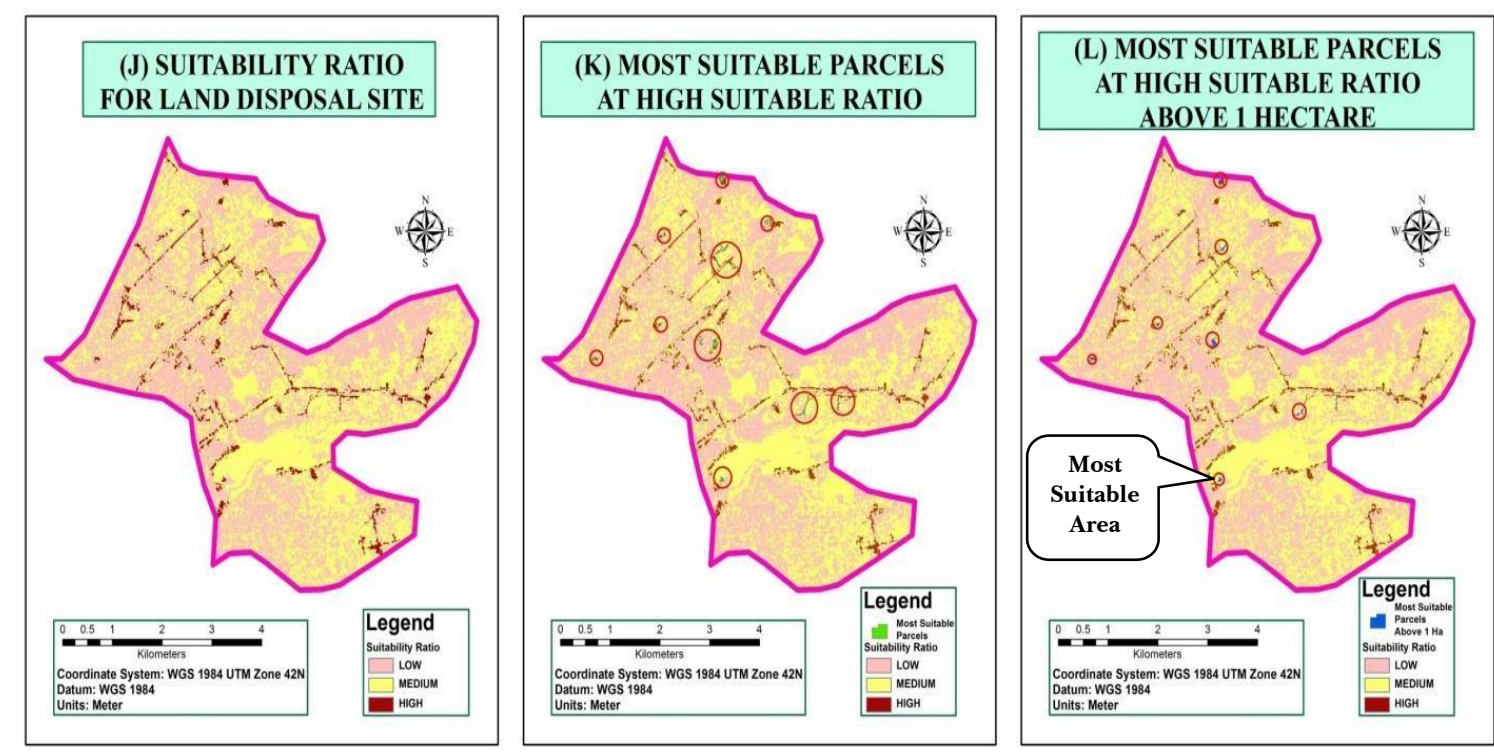

Figure 16. Maps of Suitability Indexes: (J) Suitability Ratio for Land Disposal Site (K) Most Suitable Parcels at High Suitable Ratio (L) Most Suitable Parcels at High Suitable Ratio above $1 \mathrm{Ha}$.

\section{CONCLUSION}

Spatial Decision Support System (SDSS) helps in decision making and Analytic Hierarchy processes that are suitable for organizing complex decisions resulting in appropriate site selection for solid wastes which are believed to be far away from urban population. In this study, through the technique of Analytic Hierarchy Process (AHP) and Multi-Criteria Decision Making (MCDM), suitable alternative site for dumping solid waste has been selected on the basis of different factors performed on Arc Map 10.3, Erdas Imagine, e-Cognition developer for fulfillment of decision maker's expectations. After performing different investigations on the suitability indexes, the analyzed area most suitable for solid waste dumping came out to be $200 \mathrm{~m}$ away from Malir River.

\section{REFERENCES}

[1] Haq Nawaz Abbasi, Xiwu Lu and Guangyu Zhao "An Overview of Karachi Solid Waste Disposal Sites and Environs" Journal of Scientific Research \& Reports 6(4); Article no.JSRR.2015.155 ISSN: 2320-022 pp 294-303, (2015)

[2] K. Anitha, S.Janani, A.Hemanthra, R Bhavani, L. "Iyappan Optimum route analysis to drinking water pipeline for vellore Taluk Using GIS and Remote Sensing" International Conference on Engineering Materials and Processes (ICEMAP2017, (2017) March. 
[3] Genemo Berisa (M.Sc. GIS ) and Yohanis Birhanu (M.Sc. Environmental Science) "Municipal Solid Waste Disposal Site selection of Jigjiga Town Using GIS and Remote Sensing Techniques, Ethopia" International Journal of Scientific and Research Publications, 1 ISSN 2250-3153 Volume 5, Issue 4, April (2015).

[4] Thomas, Lang, Stefan, Hay, Geoffrey "Object-based image analysis: spatial concepts for knowledge-driven remote sensing applications: Springer Science \& Business Media” Springer-Verlag Berlin Heidelberg, Edition Number 1, 2008, Book ISBN 978-3-540-77058-9, DOI 10.1007/978-3-540-77058-9.

[5] Katja Buhrkal, Allan Larsen, Stefan Ropke "The waste collection vehicle routing problem with time windows in a city logistics context" The Seventh International Conference on City Logistics 39 pp 241 - 254 (2012)

[6] Asha Poorna C, Vinod P. G "Solid waste disposal site selection by data analysis using GIS and Remote sensing tools: A case study in Thiruvananthapuram corporation area” INTERNATIONAL JOURNAL OF GEOMATICS AND GEOSCIENCES, Volume 6 Issue 4, pp 1734- 1747, (2016)

[7] Chabuk A, Al-Ansari N, Hussain HM , Knutsson S , Pusch R "Landfill site selection using geographic information system and analytical hierarchy process: A case study Al-Hillah Qadhaa, Babylon, Iraq” OSAGE Journal, volume 34(5), pp 42737, May ,(2016)

[8] Ali Jalil Chabuk1, Nadhir Al-Ansari1, Hussain Musa Hussain, Sven Knutsson1, Roland Pusch "Landfill Sites Selection Using Analytical Hierarchy Process and Ratio Scale Weighting: Case Study of Al-Mahawil, Babylon, Iraq” Engineering, volume 9, pp 123-141, February 16, (2017).

[9] Tirusew Ayisheshim Ebistu 1 and Amare Sewnet Minale “ Solid waste dumping site suitability analysis using geographic information system (GIS) and remote sensing for Bahir Dar Town, North Western Ethiopia” African Journal of Environmental Science and Technology Vol, 7(11), pp 976-989, November (2013)

[10] G.J. Hay, G. Castilla "Object-Based Image Analysis, Strengths, Weaknesses, opportunities and Treats (SWOT)" The International Archives of the Photogrammetry, Remote Sensing and Spatial Information Sciences, (2006)

[11] Oonib Azam "New garbage transfer station likely to be hazardous for Karachi" Express Tribune, March 20th, (2017)

[12] Jamshidi-Zanjani, A. \& Rezaei, M. Environ "Landfill site selection using combination of fuzzy logic and multi-attribute decisionmaking approach" Springer-Verlag GmbH Germany (2017) 16 June (2017)

[13] Cengiz Kahraman, Mehdi Keshavarz-Ghorabaee, Edmundas Kazimieras Zavadskas and Sezi Çevik "Intuitionistic fuzzy EDAS method: an application to solid waste disposal site selection" Journal of Environmental Engineering and Landscape Management, Volume 25(01) pp 1-12, March (2017). 


\section{AUTHORS}

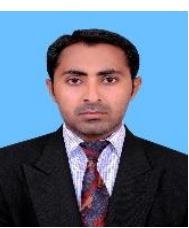

\section{Sikandar Ali}

sayed.sikandar24@gmail.com

MS (RS \& GIS) From Department of Geography University of Karachi Pakistan, Currently working as lecturer at Faculty of Engineering, Science \& Technology Indus University Pakistan

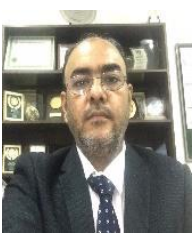

\section{Prof. Dr. Engr. Sayed Hyder Abbas Musvi}

sayed.sikandar24@gmail.com

Senior Member of IEEE

Dean at Faculty of Engineering, Science \& Technology Indus University, Karachi, Pakistan

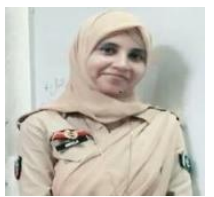

\section{Abeela Tameez}

picesfrienda18@gmail.com

MS (RS \& GIS) Student Department of Geography University of Karachi, Pakistan

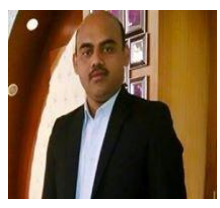

\section{Masood Alam}

masood.78@hotmail.com

MS (RS \& GIS) Student Department of Geography University of Karachi, Pakistan

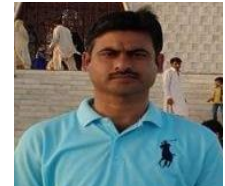

\section{Asim Nawaz}

asimpmd@gmail.com

MS (RS \& GIS) Student Department of Geography University of Karachi 
Analyzing site suitability for solid waste disposal through GIS multi-criteria decision making hierarchy process DOI: http://dx.doi.org/10.17993/3ctecno.2019.specialissue.07 


\title{
EFFECTING FACTORS OF KNOWLEDGE INTEGRATION THROUGH SOCIAL MEDIA IN SMALL MEDIUM ENTERPRISES ENVIRONMENT
}

\author{
Nur Ilyana Ismarau Tajuddin \\ Faculty Computer Science and Information Technology, University Putra, (Malaysia) \\ E-mail: ilyanaismarau90@gmail.com
}

Rusli Abdulllah

Faculty Computer Science and Information Technology, University Putra, (Malaysia)

E-mail: rusli@upm.edu.my

Marzanah A. Jabar

Faculty Computer Science and Information Technology, University Putra, (Malaysia)

E-mail: marzanah@upm.edu.my

Yusmadi Yah Jusoh

Faculty Computer Science and Information Technology, University Putra, (Malaysia)

E-mail:yusmadi@upm.edu.my 


\section{ABSTRACT}

Knowledge Integration (KI) has been significant concern in analyzing the organization performance. KI also has been challenged by the emergence and continued development technologies that have made new sources of information and knowledge available to Small Medium Enterprises (SMEs). Social media are widely adopted by organizations to enhance the effectiveness of KI practices. The purpose of this study to investigate the factors that influence of KI through social media. Based on the 11 selected papers, this review has established 12 factors that may influence of knowledge integration through social media. Hence, this research contribute to the practice and research of KI and social media and its effectiveness towards the organizational performance.

\section{KEYWORDS}

Knowledge Integration, Social Media, Small Medium Enterprise, Systematic Literature Review.

\section{INTRODUCTION}

In the new age, knowledge has been recognized as one of most valuable assets which developed in organization. Knowledge is related to activities and process flow in and between the organizations. Knowledge integration is solitary of the most essential approaches of knowledge applications to attain sustainable competitive advantages and business value [1]. Only the organization has the capabilities of integrating the inside and outside resources to innovate faster, would be able to succeed under the ultra-competitive environment [2]. From the time when knowledge is continually changing and depreciating, organizations cannot possess all the required knowledge by themselves. The key is to utilize expertise that is spread within the enterprise by integrating knowledge [1]. KI is required in many situations where coherent combining of disparate sources and levels of information for some enterprise is necessary [3]. Technological cooperation among firms is important because a large part of the knowledge needed in innovation processes is tacit, and can be transferred through social media interactions [4]. According to [5] the effective adoption of technologies in companies is much depending on technology characteristics, project and organizational characteristics, user and social characteristics, and task characteristics. However, in reality, these factors are much neglected by organizations, especially among small companies. Social media tools have ability to integrate all information and knowledge that can be obtained [6]. Even though reports suggest the social media tools enhance the development of SMEs, there is still little empirical evidence on their adoption and usage from the category of firms [7] especially in the KI [8].

This study aims to investigate the factors that influencing of KI and Social Media. The findings will be able to assist the SMEs manager to adapt Social Media for integrating knowledge. The following section presents the review of the methodology. Subsequently, followed by discussion section in which focused on influencing factors of KI. The final section concludes the current study by revealing the research contribution and proposing for further research possibility.

\section{REVIEW METHODOLOGY}

According to Okoli et al. [9], the SLR processes contains of three stages namely: planning, executing and reporting stage as show in Figure 1. The following sub sections will discuss the processes in details. 


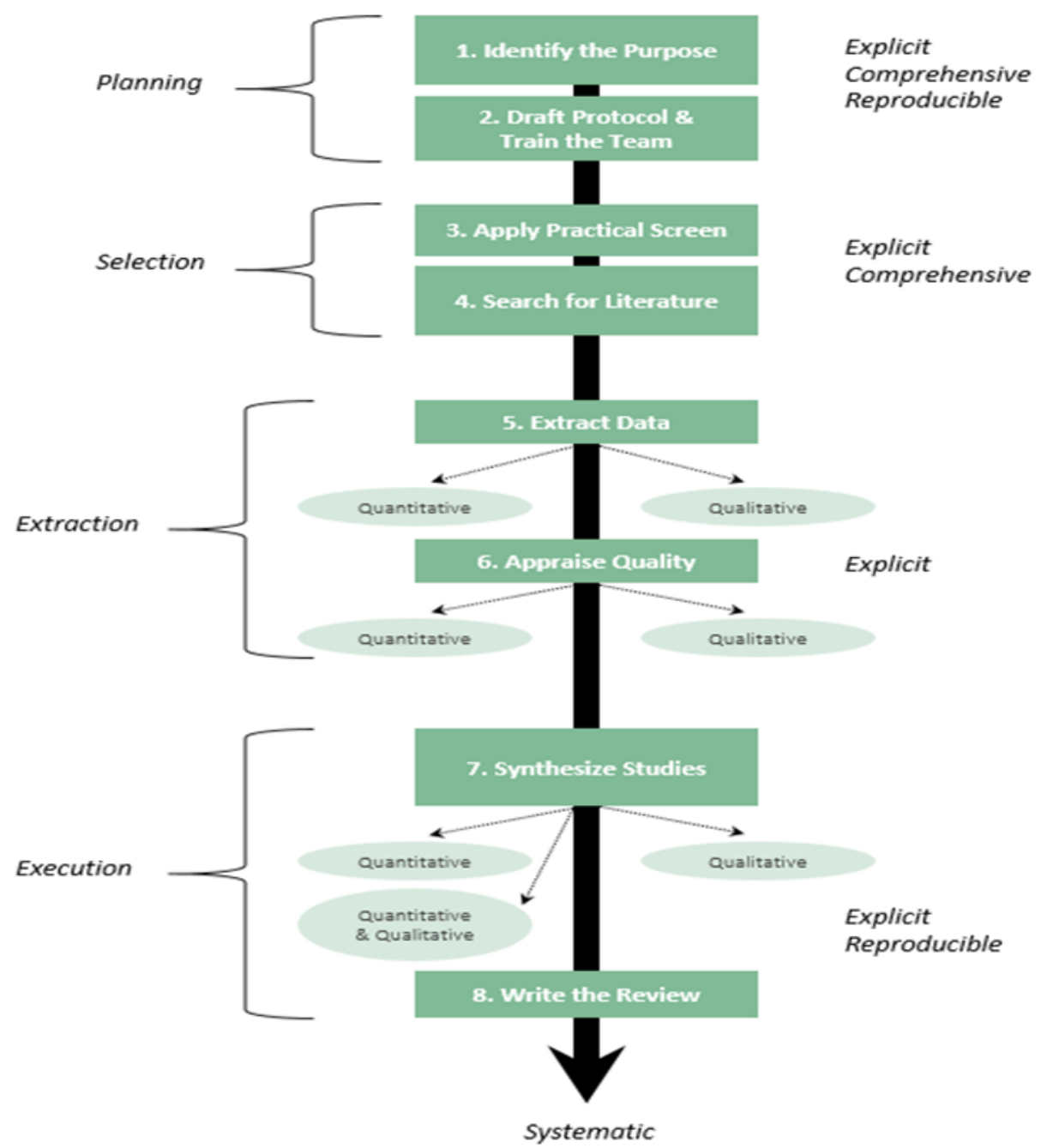

Figure 1. SLR process (Source: Okoli, 2010[9]).

\subsection{Planning Stage}

The planning stage activities include identifying specific research context, defining reviewing protocols, and constructing of research questions. The research question (RQ) is 'What are the factors that influence the KI through social media'.

\subsection{Executing Stage}

A literature search was conducted from December 2010 to September 2017 using three leading electronic journal databases. The databases are ACM, IEE, Science Direct and Scopus. The search was limited to peer-reviewed, English-language journal papers published between 1996 and 2016. Search result for SCOPUS, Science Direct, IEEE and ACM are summarize in Table 1. The search analyst titles and abstracts for a number of related keywords and phrases: "knowledge integration", "knowledge combination", "knowledge mapping", "knowledge collaboration", "social media", "technology", "social network", "organization", "Small Medium Enterprises" etc. When searching in the databases, therefore, we use three couples of combinative keywords: (knowledge AND integration), (knowledge AND combination) and (knowledge AND collaboration) etc. The result is shown as Table 2.

Certain criteria have been set in choosing the articles. The article was only included if it met the following criteria. Firstly, its focus was primarily on KI/ Social media. Secondly, 
the area investigated included SMEs environment. Finally if only the article are discussed factors effect on KI through Social Media. The current research focus merely in peerreviewed primary studies published in academic journals were included, resulting in the exclusion of literature reviews, conceptual articles, conference proceedings. The articles which full filled the above-mentioned three criteria were scanned by the authors for additional studies that met the inclusion criteria for the review. In this stage, the duplicated articles were removed. Further, the articles were screened for relevance, primarily based on the title and abstract.

Table 1. Search Result based on Year.

\begin{tabular}{|l|l|l|l|l|}
\hline Year Search & Scopus & $\begin{array}{l}\text { Science } \\
\text { Direct }\end{array}$ & IEEE & ACM \\
\hline $\mathbf{2 0 1 7}$ & 70 & 156 & 300 & 6 \\
\hline $\mathbf{2 0 1 6}$ & 98 & 267 & 354 & 4 \\
\hline $\mathbf{2 0 1 5}$ & 125 & 273 & 341 & 4 \\
\hline $\mathbf{2 0 1 4}$ & 188 & 200 & 484 & 2 \\
\hline $\mathbf{2 0 1 3}$ & 183 & 182 & 426 & 5 \\
\hline $\mathbf{2 0 1 2}$ & 190 & 166 & 458 & 1 \\
\hline $\mathbf{2 0 1 1}$ & 192 & 140 & 500 & 1 \\
\hline $\mathbf{2 0 1 0}$ & 122 & 272 & 340 & 4 \\
\hline
\end{tabular}

Table 2. Search Result based on Keyword.

\begin{tabular}{|l|c|c|c|}
\hline & $\begin{array}{c}\text { knowledge } \\
\text { AND } \\
\text { integration }\end{array}$ & $\begin{array}{c}\text { knowledge } \\
\text { AND } \\
\text { combination }\end{array}$ & $\begin{array}{c}\text { knowledge } \\
\text { AND } \\
\text { collaboration }\end{array}$ \\
\hline Scopus & 7 & 13 & 35 \\
\hline $\begin{array}{l}\text { Science } \\
\text { Direct }\end{array}$ & 90 & 56 & 130 \\
\hline IEEE & 2200 & 1745 & 1955 \\
\hline ACM & 3 & 3 & 6 \\
\hline
\end{tabular}

The selected papers were analyzed and synthesized before findings and results be discussed in the following section. This was followed by the quality assessment screening. The quality assessment was formulated to evaluate the completeness of papers and advantageous for data extraction [10]. These four questions (Q1-Q4) are presented in Table 3. Each question has only three answer options: Yes $=1$; Partially $=0.5$; and $\mathrm{No}_{0}=0$.

Table 3. Quality Assessment Criteria.

\begin{tabular}{|l|l|l|}
\hline No. & Item & Answer \\
\hline Q1 & $\begin{array}{l}\text { Is there a clear description of the aims and objectives of the } \\
\text { investigation? }\end{array}$ & Yes/No \\
\hline Q2 & $\begin{array}{l}\text { Is the paper explained the method of analysis pertinent and } \\
\text { adequately? }\end{array}$ & $\begin{array}{l}\text { Yes/No/ } \\
\text { Partially }\end{array}$ \\
\hline Q3 & $\begin{array}{l}\text { Is the paper supported by primary data? } \\
\text { Yes/No }\end{array}$ & Yes/No/Partially \\
\hline Q4 & Is the paper explained the model structure in detail? & \\
\hline
\end{tabular}

\subsection{Reporting Stage}

In reporting stage, the findings and results were discussed in section 3 . 


\section{FINDING AND ANALYSIS}

A total of 259 references that are deemed relevant to this topic. In the next stage, the abstract and brief content of selected paper was evaluated. The 40 relevant papers were then filtered by applying the quality assessment criteria. In the very final round, only 11 papers out of 40 papers $(27.5 \%)$ were accepted for data synthesis of evidence after executing exclusion criteria. The summary of the quality assessment of the 11 papers (A1-A11) considered for this review as show in Table 4.

Table 4. Quality Assessment Result.

\begin{tabular}{|l|l|l|l|l|l|}
\hline ID & Q1 & Q2 & Q3 & Q4 & Total \\
\hline A1 & 1 & 0 & 0 & 1 & 2 \\
\hline A2 & 1 & 1 & 1 & 1 & 4 \\
\hline A3 & 1 & 0.5 & 0 & 1 & 2.5 \\
\hline A4 & 1 & 0.5 & 0 & 1 & 2.5 \\
\hline A5 & 1 & 1 & 1 & 1 & 4 \\
\hline A6 & 1 & 1 & 1 & 1 & 4 \\
\hline A7 & 1 & 1 & 1 & 1 & 4 \\
\hline A8 & 1 & 1 & 1 & 0 & 3 \\
\hline A9 & 1 & 1 & 1 & 1 & 4 \\
\hline A10 & 1 & 1 & 1 & 1 & 4 \\
\hline A11 & 1 & 1 & 1 & 1 & 4 \\
\hline
\end{tabular}

Table 5 exemplifies the filtering result of the quality assessment of all that paper that was classified as good and very good scores. Among the 11 selected papers, three papers has scored good quality in which consists of 27 percentage and eight papers scored a very good quality scoring with 73 percentages.

Table 5. Quality Scores.

\begin{tabular}{|c|c|c|c|c|c|}
\hline Quality Scale & $\begin{array}{c}\text { Very poor } \\
(<1)\end{array}$ & $\begin{array}{c}\text { Poor } \\
(1-<2)\end{array}$ & $\begin{array}{c}\text { Good } \\
(2-<3)\end{array}$ & $\begin{array}{c}\text { Very good } \\
(3-4)\end{array}$ & Total \\
\hline Number of studies & 0 & 0 & 3 & 8 & 11 \\
\hline Percentage (\%) & 0 & 0 & 27 & 73 & 100 \\
\hline
\end{tabular}

These 11 papers were investigated in the area of Knowledge Integration area is categorized in three types of research analysis includes conceptual, empirical and case study. Remarkably, there were seven publication of empirical studies published from the year 2011 until the year of 2017. Further, followed by three conceptual paper published from 2010-2012 and a case study in 2015. The KI publication trend is presented in Figure 2 and Figure 3. 


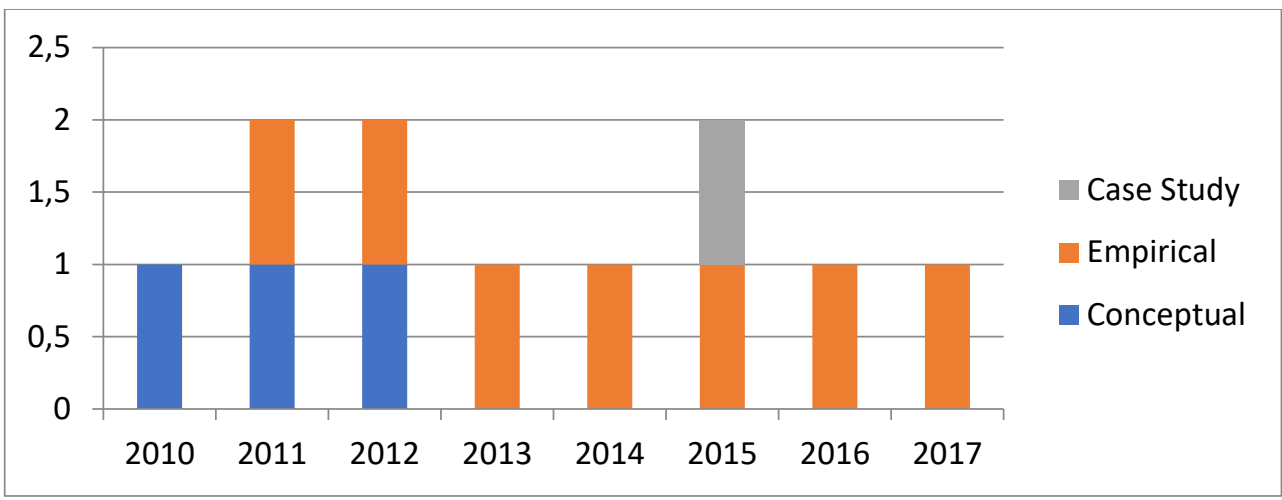

Figure 2. Paper distribution by publication year.

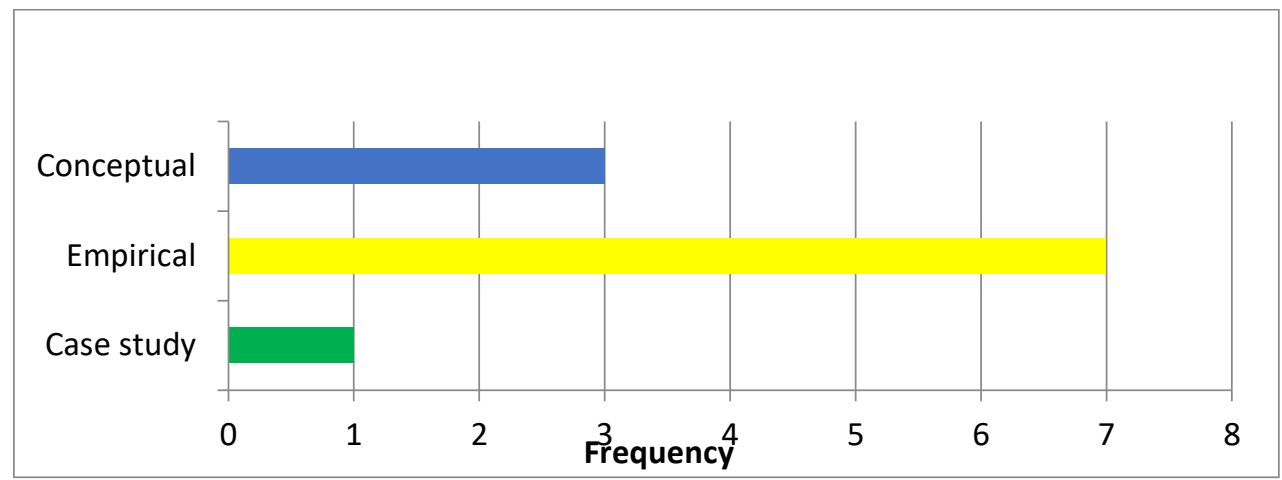

Figure 3. The frequency of research analysis.

Figure 4 displays the KI studies based on domain specific. Nine papers (82\%) are from various organizations only 1 paper $(9 \%)$ from manufacturing sector and healthcare. Table 6 display the summary of the relationship of the influencing factors in KI.

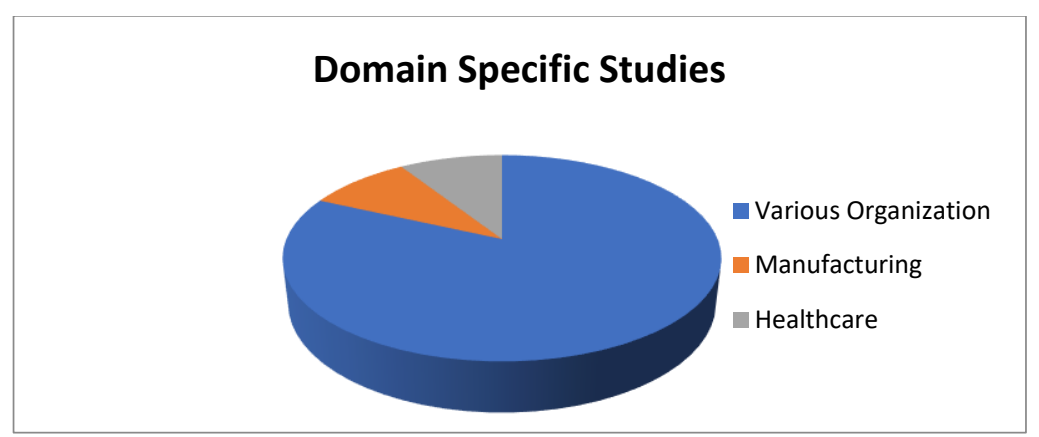

Figure 4. Domain specific studies.

Table 6. Summary of the Relationship of the Influencing Factors in KI.

\begin{tabular}{|l|l|c|c|c|}
\hline ID & $\begin{array}{c}\text { Type of } \\
\text { research }\end{array}$ & Sample size & Domain & Source \\
\hline A1 & Conceptual & N/A & $\begin{array}{c}\text { Various } \\
\text { organization }\end{array}$ & {$[11]$} \\
\hline A2 & Empirical & $\begin{array}{c}\text { Various } \\
\text { organization } \\
\text { manager, senior, CEO }\end{array}$ & $\begin{array}{c}\text { Various } \\
\text { organization }\end{array}$ & {$[12]$} \\
\hline A3 & Conceptual & ABC Co Ltd & $\begin{array}{c}\text { Various } \\
\text { organization }\end{array}$ & {$[14]$} \\
\hline A4 & Conceptual & Multi-vendor & \\
\hline
\end{tabular}




\begin{tabular}{|c|c|c|c|c|}
\hline A5 & Empirical & $\begin{array}{l}157 \text { full time professional } \\
\text { from variety industry }\end{array}$ & $\begin{array}{c}\text { Various } \\
\text { organization }\end{array}$ & {$[15]$} \\
\hline A6 & Empirical & $\begin{array}{l}262 \text { Chinese working } \\
\text { professional }\end{array}$ & $\begin{array}{c}\text { Various } \\
\text { organization }\end{array}$ & {$[8]$} \\
\hline A7 & Empirical & $\begin{array}{c}182 \text { Taiwan Manufacturing } \\
\text { Firm }\end{array}$ & Manufacturing & [16] \\
\hline A8 & Case Study & ABC Company & $\begin{array}{c}\text { Various } \\
\text { organization }\end{array}$ & [17] \\
\hline A9 & Empirical & 265 high technology firm & $\begin{array}{c}\text { Various } \\
\text { organization }\end{array}$ & [18] \\
\hline A10 & Empirical & 317 clinic staff & Healthcare & [19] \\
\hline A1 1 & Empirical & 114 worker from China & $\begin{array}{c}\text { Various } \\
\text { organization }\end{array}$ & [20] \\
\hline
\end{tabular}

Grounded on prior research as mention in Table 6, number of 12 factors have been emerged as factors that influences the KI as illustrated in Table 7.

Table 7. Influencing Factors of KI Studies.

\begin{tabular}{|c|c|c|}
\hline No & Factors & Articles \\
\hline 1 & Organizational Learning & A2, A4 \\
\hline 2 & Social Capital & A6, A4 \\
\hline 3 & Social network & A5, A8,A1,A3 \\
\hline 4 & IT Capability & A2 \\
\hline 5 & Media interactive & A8 \\
\hline 6 & Competitive industry & A9 \\
\hline 7 & Market turbulence & A9 \\
\hline 8 & Technology turbulence & A9 \\
\hline 9 & Knowledge integration mechanism & A7, A9, A4 \\
\hline 10 & Integrative Capability & A1 \\
\hline 11 & Transactive Memory System & A1, A5, A8, A3, A11 \\
\hline 12 & Teamwork & A10 \\
\hline
\end{tabular}

Figure 5 presents the frequency of influencing factors of KI studies. KI factors comprises of Organizational Learning (2 papers), Social Capital (2 papers), Social Network (4 papers), IT Capability (1 paper), Media Interactive (1 paper), Competitive Industry (1 paper), Market Turbulence (1 paper), Technology Turbulence (1 paper), Knowledge Integration Mechanism (3 papers), integrative Capability (1 paper), Transactive Memory System (5 papers) and Teamwork (1 paper). Based on Table 5 and Figure 4 , the detail of each factors will be discuss in the discussion section. 


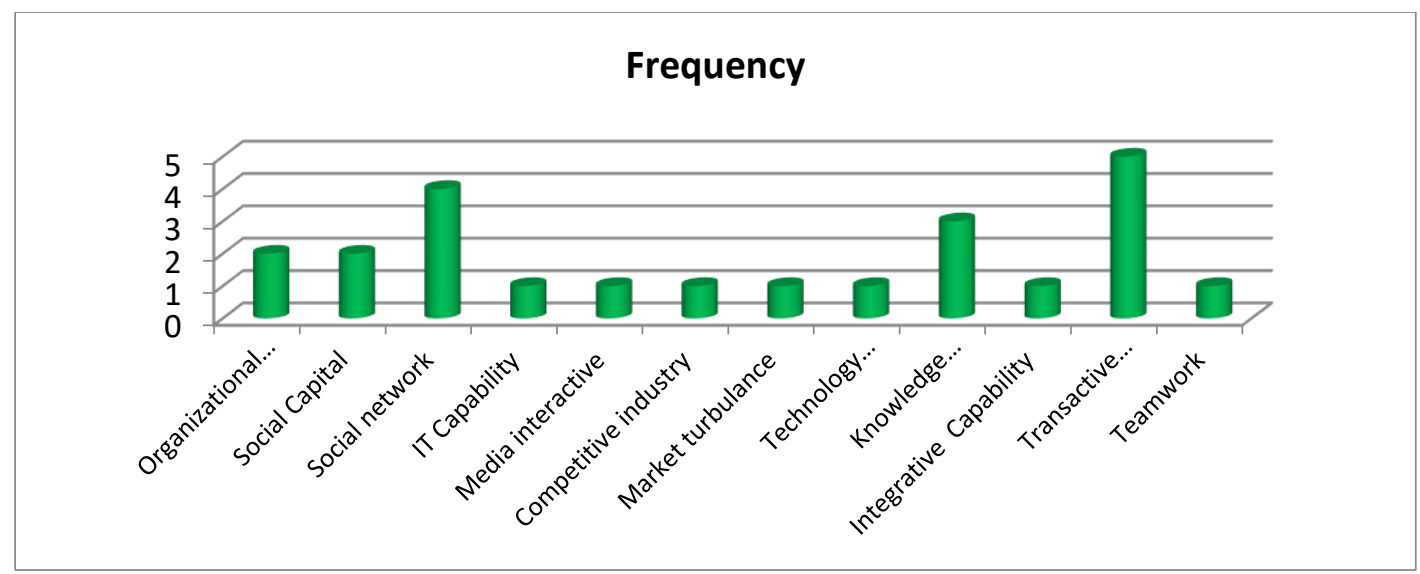

Figure 5. Frequency of influence factors of KI.

\section{DISCUSSION}

Based on prior research, there are 12 factors that are effecting of KI:

\subsection{Organizational Learning}

Organizational learning refers as an organization's developing new thinking and creating new knowledge to enhance prevailing resources [21]. On the other hand, organizational learning as gaining knowledge, skill, value, belief in improving growth and developing the organization. Organizational learning have effect to KI to advance the organization performances $[12,14]$. Thus, organizational learning is significant considered factor to improve SMEs performances.

\subsection{Social Capital}

Social capital refers as the networks of relationships among people who live and work in a particular society, enabling that society to function effectively. Social capital inheres in the relationships between actors within the social network [22]. The dimensions consist in social capital is structural [14] relational and cognitive [8]. Social capital is the significant effect to be considered for organization performances.

\subsection{Social Network}

The social network is the level of connectivity and access among individuals in organization to enable communication, dialogue and interaction between organizations to integrate knowledge [23]. Social network do has effect on the KI [11, 13, 15, and 17]. Social network have been developed and have been applied to knowledge processing. Thus, social network is substantial to be considered in knowledge integration.

\subsection{IT Capability}

IT capability refers as the ability to effectively manage the hardware and software that has different type and levels of knowledge [12]. IT capability allows user to communicate with each other, facilitate knowledge acquisition and integration, easily reach expert in specialized areas, and foster boundary straddling activities [24]. Thus, IT Capability is significant to consider in knowledge integration in SMEs.

\subsection{Media Interactive}

In other hand, by amending a form and the contents of it the user are able to create the mediating affect of the environment on the moment, in which define the media interactive. Media interactive include five crucial elements. The components are: (a) 
amount of acceptable input, (b) the number and type of characters that are able to be applied changes, (c) the distance of ability to receive message; the capability to integrate, build, and reconfigure inner and outer knowledge to response to environmental change [28]. Media interactivity has an important role in social media. Prior research [17] has clearly found media interactivity to have a positive relationship to knowledge integration in the organization.

\subsection{Competitive Industry}

Competitive industry is the degree of competition that a firm faces in the respective industry [26]. Competitive industry has relationship to KI in organization [18].

\subsection{Market Turbulence}

Market turbulence is the rate at which customer composition and customer preferences change. Market turbulence has relationship to KI in the organization [18]. Although there are few studies on the relationship between market turbulence and KI has shown that market turbulence positively influences. Thus, market turbulence is significant consider effect to KI.

\subsection{Technology Turbulence}

Technology turbulence is the rate of change in technologies involved in the development of new product. In order to enhancing organization performance, an organization's willingness to explore new technologies for product or service innovation is likely to depend on the opportunities or threats arising from environmental changes Technology turbulence has a relationship to KI in the organization [18]. Although there are few studies on the relationship between technology turbulence and KI has shown that technology turbulence positively influences. Thus, technology turbulence is significant consider effect to knowledge.

\subsection{Knowledge Integration Mechanism (KIM)}

Knowledge Integration Mechanism (KIM) are structures and processes for integrating the different types of knowledge among the different functional unit in the organization [27]. KIM consist of three perspectives, namely systems and procedural mechanisms, interactive mechanisms and boundary-crossing mechanisms. Prior studies [14, 16, and 18 ] suggested KIM has effect to KI in the organization performances and new product development.

\subsection{Integrative Capability}

Integrative capability refers to a firm's ability to acquire, combine and deploy resources. Prior research on dynamic capabilities and combinative capabilities presents insight into the relationship between organization's integrative capability and knowledge integration. Dynamic capability refers to an ability to integrate, build, and reconfigure internal and external knowledge to respond to environmental change [28]. Integrative capability, including ability to enter and gain external new knowledge. The capability to recognize internal knowledge are in position to achieve a total new positive relation to knowledge integration [17].

\subsection{Transactive Memory System (TMS)}

Transactive Memory System (TMS) refers as cooperative division of labor for remembering, learning, and communicating relevant knowledge [29]. TMS is the 
mechanisms that can help team's member recognize other's expertise. Team members can rely on each other's expertise to complete a task based on specific domain. TMS consists of three construct which are specialization, credibility and coordination. TMS also has an important role in social media. TMS is posited to have positive relations to KI $[12,13,15,17$, and 20]. Therefore, TMS is acknowledging as key factor of KI through social media.

\subsection{Teamwork}

The team are viewable as a knowledge integrating mechanisms, individual knowledge can be shared and mobilized in the team [30]. The empirical study has undoubtedly found that the effective teamwork are required in KI [19] in order to emergent process of rich exchanges and joint problem solving to integrate and apply knowledge and expertise to the task at hand in a coordinated manner [31].

\section{CONCLUSION IN FUTURE WORK}

This study provided insight on influence factors of KI and social media, which important determinant of SMEs performances. A total ten relevant papers were thoroughly reviewed and analyze. The review process has identified and categorized the influenced factors of KI. There are twelve factors which have been identified to influence of KI. These factors includes: Organizational Learning, Social Capital, Social Network, IT Capability, Media Interactive, Competitive Industry, Market Turbulence, Technology Turbulence, Knowledge Integration Mechanism, integrative Capability, Transactive Memory System and Teamwork. The factors of KI and social media necessity to authenticate and validate through further research in order to gain better insights of the current research.

\section{REFERENCES}

[1] Grant, R.M. Toward a knowledge-based theory of the firm. Strategic Management Journal, 17 (S), 109- 122 (1996).

[2] Gao Wei, Wang Hengshan, N.-W. Bin. Knowledge Integration and Its Impact.In International Conference on Managment Science \& Engineering. 1517-1523 (2007).

[3] Hustad, E. A Conceptual Framework for Knowledge Integration in Distributed Networks of practice. Proceedings of the 40th Hawaii International Conference on System Sciences - 2007, 1-10 (2007).

[4] Raban, Y. 12 Supporting Knowledge Integration at SMEs - Policies Profiles of KI Support Measures for SMEs. (J. K. Antonie Jetter, Ed.). Physica Verlag - A Springer Company (2008).

[5] Petter, S., DeLone, W., \& McLean, E. R. Information Systems Success: The Quest for the Independent Variables. Journal of Management Information Systems, 29(4), 7-61 (2013).

[6] Fung, C. K., \& Hung, P. C. Information and knowledge management in online rich presence services. Information Systems Frontiers, 1-3 (2013).

[7] Dixon, Brian E. Towards E-Government 2.0: An Assessment of Where EGovernment 2.0 Is and Where It Is Headed. Public Administration \& Management, 15(2), 418-454 (2010). 
[8] Cao, X., Guo, X., Liu, H., \& Gu, J. The role of social media in supporting knowledge integration: A social capital analysis. Information Systems Frontiers, 1-12 (2013).

[9] Okoli, Chitu, and Kira Schabram. "A guide to conducting a systematic literature review of information systems research." (2010).

[10] Adrian, R. Abdullah, R. Atan and Y. Y. Jusoh, "Factors influencing to the implementation success of big data analytics: A systematic literature review," 2017 International Conference on Research and Innovation in Information Systems (ICRIIS), Langkawi, 2017, pp. 1-6.

[11] Hong, D. C., \& Liang, S. Media Characteristics and Social Networks-enabled Knowledge Integration in Cooperative Work.Procedia Computer Science, 60, 246-255 (2015).

[12] Kim, Y. J., Song, S., Sambamurthy, V., \& Lee, Y. L. Entrepreneurship, knowledge integration capability, and firm performance: An empirical study. Information Systems Frontiers, 14, 1047-1060 (2011).

[13] Ling, Hong, Dao Cheng Hong, and Cheng Hong Zhang. "Research on tacit knowledge integration: a synthesis of social ties and TMS." Knowledge Management Research \& Practice 9.3 :256-262 (2011).

[14] Jin, Xiaowei, and Julia Kotlarsky. "A Conceptual Framework of Knowledge Integration in Multisourcing Arrangements." (2012). 33th International Conference on Information System, Orlando. 2012.

[15] Zhang, C.H., Hong, D., Ling, H. A Study On The Mechanism Of Tacit Knowledge Integration: The Role Of Social Ties And Transactive Memory Systems. International Journal of Innovative Computing, Information and Control, 8(8), 5847-5857 (2012).

[16] Tsai, Kuen-Hung, and Teresa Tiaojung Hsu. "Cross-Functional collaboration, competitive intensity, knowledge integration mechanisms, and new product performance: A mediated moderation model." Industrial Marketing Management 43.2 : 293-303 (2014).

[17] Hong, D. C., \& Liang, S. Media Characteristics and Social Networks-enabled Knowledge Integration in Cooperative Work. Procedia Computer Science, 60, 246255 (2015).

[18] Tsai, K.-H., Liao, Y.-C., \& Hsu, T. T. Does the use of knowledge integration mechanisms enhance product innovativeness? Industrial Marketing Management, 46, 214-223 (2015).

[19] Korner, M., Corinna, L., Becker, S., Lars, R., Christian, M., \& Linda, Z. Knowledge Integration, teamwork and performance in health care. Journal of Health Organization and Management, 30(2), 227-243 (2016).

[20] Hong, D., \& Zhang, L. ScienceDirect Does Transactive Memory Systems Promote Knowledge Integration Directly? Procedia Computer Science, 112, 896-905 (2017).

[21] Kessel, F. G. A. Van. No Creative Person is an Island: Organisational Culture , Acedemic Project Based Creativity, Mediating role of Intraogranisational Social Ties. SAJEMS Special Issues, 17(17), 46-69 (2014).

[22] Coleman, J. S. Social capital in the creation of human capital. The American Journal of Sociology, 94,95-120 (1988). 
[23] Chow, W. S., \& Chan, L. S. Social network, social trust and shared goals in organizational knowledge sharing. Information \& Management, 45(7), 458-465 (2008).

[24] Dewett, T., \& Jones, G. The role of information technology in the organization: a review, model, and assessment. Journal of Management, 27(3), 313-346 (2001).

[25] Lombard, Matthew, Jennifer Snyder-Duch, and Cheryl Cam- panella Bracken. 2002. "Content Analysis in Mass Communication: Assessment and Reporting of Intercoder Reliability.” Human Communication Research 28 (4): 587- 604.

[26] Cui, A. S., Griffith, D. A., \& Cavusgil, S. T. The influence of competitive intensity and market dynamismon knowledgemanagement capabilities of multinational corporation subsidiaries. Journal of International Marketing, 13(3), 32-53 (2005).

[27] De Luca, L. M., \& Atuahene-Gima, K. Market knowledge dimensions and crossfunctional collaboration: Examining the different routes to product innovation performance. Journal of Marketing, 71(1), 95-112 (2007).

[28] Zollo, M., \& Winter, S. G. Deliberate learning and the evolution of dynamic capabilities. Organization Science, 13(3), 339-351 (2002).

[29] Hollinshed A. B., "Communication, learning, and retrieval in transactive memory systems", Journal of Experimental Social Psychology, 34 (5) .423-442 (2002).

[30] Erhardt, N. Is it all about teamwork? Understanding processes in team-based knowledge work. Management Learning, 42(1), 87-112 (2011).

[31] Alavi, Maryam, and Amrit Tiwana. "Knowledge integration in virtual teams: The potential role of KMS." Journal of the Association for Information Science and Technology 53.12: 1029-1037 (2002). 


\title{
AN OPTIMIZED HYBRID BEAMFORMING FOR MILLIMETER WAVE MU- MASSIVE MIMO SYSTEM
}

\author{
Saeed Ali Saeed Alquhaif \\ University of Lahore, Lahore, (Pakistan) \\ E-mail: s.alquhaif@gmail.com \\ Ishtiaq Ahmad \\ University of Lahore, Lahore, (Pakistan) \\ E-mail: ishtiaq.ahmad@ee.uol.edu.pk \\ Mahnoor Rasheed \\ University of Lahore, Lahore, (Pakistan) \\ E-mail: mahnoor.rasheed@es.uol.edu.pk \\ Ali Raza \\ University of Lahore, Lahore, (Pakistan) \\ E-mail: ali.raza@ee.uol.edu.pk
}




\section{ABSTRACT}

Millimeter wave (mmWave) and massive multiple-input multiple-output communications (massive MIMO) have been adopted as the most important keys for the next generation (5G) wireless networks due to high spectrum availability and massive use of space diversity for high throughput communications. Massive MIMO must be implemented parallel with mmWave to overcome high path-loss issue in mmWave band by improving the antennas gain. Digital precoding in massive MIMO shows high performance at the cost of hardware complexity due to the requirement of huge number of radio frequency (RF) chains and analog-to-digital converters (ADCs). On the other hand, analog precoding has less complexity with limited performance as it supports only one data stream. In compromise between these two, a hybrid precoding for multiuser massive MIMO (MU-massive MIMO) systems with low-complexity and reasonable performance becomes necessary for next generation networks. We propose an optimized hybrid precoding and combining scheme for fully connected structure. The design of combined analog and digital precoding for the mmWave MU-massive MIMO system becomes a non-convex problem. Therefore, we proposed a suboptimal design, where we split the transmitter and receiver hybrid precoding and decoding and adopt orthogonal matching pursuit algorithm for their design. The simulation results show that the performance of proposed hybrid precoding approaches the digital precoding in the term of total sum data rate as we increase number of RF chains and SNR.

\section{KEYWORDS}

Hybrid precoding, beamforming, MU-MIMO, Massive MIMO.

\section{INTRODUCTION}

Scarcity of spectrum has always been a problem in communication networks. Researchers always look adequate band for good propagation properties. Unfortunately, the current spectrum does not fulfill the need of bandwidth greedy broadband communications. For future wireless system such as $5 \mathrm{G}$, data rates for a user expected to increase explosively. Therefore, the researcher is working to move their attention to millimeter wave (mmWave) communications from microwave communications due to the availability of a huge amount of unused spectrum at mmWave. Communication at mmWave is targeting a speed in Gbits/s (data rates about $10 \mathrm{Gbits} / \mathrm{s}$, which is more than current 4G 100 times) according to the IEEE 802.11 and WiGig. However, operating at mmWave comes with the high signal attenuation and scattering limitations. To overcome the issue of signal attenuation many researchers have proposed a new small cell system like micro-cell, femto-cell and pico-cell. By the small cells, we can combine mmWave and MIMO system to perform 3D beamforming easily. Directional communication which requires transmit and receive antenna beamforming can improve signal-to-noise ratio (SNR) to the sufficient level in the mmWave bands. Implementation of the massive MIMO at mmWave for beamforming enables us to install hundreds or thousands of antennas at the base station and receiver, which increases SNR at the receiver, consequently, a significant increase in system capacity can be observed. With digital beamforming, multiple streams can be transmitted in parallel, however it requires large numbers of radio frequency (RF) chains and analog to digital converters (ADCs) which makes the digital beamforming more complex and costly in the term of power consumptions. While analog precoding has less complexity and can be implemented with the phase shifter that controls the phases of transmit signal, but it supports only one data stream with high SNR, resultantly the capacity cannot be increased significantly. However, combining of both the schemes 
analog and digital precoding, enables us to get the advantages of the both. Therefore, hybrid beamforming becomes a hot research issue [1].

While designing precoding schemes for mmWave massive MIMO system, mostly researchers consider fully connected structure [2]. Many algorithms are used for different structures for hybrid precoding. Assuming perfect channel knowledge at the transmitter, a low-complexity iterative algorithm based on orthogonal matching persuit (OMP) is proposed in [3]. The hybrid technique provides a trade-off between the analog precoding that offers low-complexity with limited-performance and digital precoding having high-complexity with good-performance [4][5]. One of the most advantages of hybrid scheme is that it can deal with the less number of RF chains and more number of antennas [6]. In [7], the spares nature of the mmWave channel is studied. Following [6], the authors in [2] designed hybrid precoding algorithm based on alternative minimization and matrix decomposition. In [8] system model investigates both fully connected structure (each antenna is connected to all RF chains) and partially connected structure. Based on spatially sparse channel, a hybrid precoding is proposed in [9] that solves the problem by spares approximation approach for achievable rate as the nearoptimal solution. A codebook based algorithm is proposed in [10] that involve an iterative search in predefined codebook to get the matrix of optimal hybrid precoding.

In the existing research, the most works on hybrid beamforming are focusing on single user MIMO (SU-MIMO) systems and trying to reduce the RF chains without affecting the multi-data streams. It is found that, the number of RF chains are still more than data streams, which makes the hybrid beamforming hard to be implemented and costly in the term of the power consumption. In our work, we proposed a novel hybrid precoding where a group of antenna serves a group of users simultaneously as a result the required number of RF chain are reduced. We design hybrid precoding for multiuser massive MIMO (MU-massive MIMO) in mmWave system as follows:

(i) First we design analog and digital decoder for each user.

(ii) Use effective channel to calculate analog precoder, which is same for all the users.

(iii) Adopt block diagonalization (BD) for mitigate the interference and intrainterference between the users.

(iv) By some available algorithms, we design analog RF precoder system.

(v) Digital precoding designed by $\mathrm{BD}$ maximizes the total rate for multiuser massive MIMO system in downlink channel.

(vi) Hybrid beamforming has been design by weighted sum mean Square error (WSMSE) for which is the inverse gain of its digital precoding.

(vii) Finally, we study the performance of both digital and proposed hybrid beamforming with different parameters like SNR, data streams and RF chains.

The rest of the paper is organized as follows. Massive MIMO and mmWave communications are studied in detail in Section 2. System model and channel model are described in Section 3. The hybrid precoding design is explained in Section 4, while Section 5 narrates simulation results. Finally, the conclusions are made in Section 6. 


\section{MASSIVE MIMO AND MILLIMETER WAVE COMMUNICATIONS}

MIMO has become integral part of wireless standards as it can increase the reliability and throughput of the wireless communication systems [11] and one of the most important key for current $4 \mathrm{G}$ wireless systems. Initially, MIMO was designed for the single-user, therefore, SU-MIMO become the integral part of long-term evaluation and WiMAX [12]. Later it shifted from single-user to multi-user MIMO system [12] where base station can communicate with a group of users simultaneously with multiple antennas. To meet increasing data rates and traffic demand, currently, massive MIMO has become hot research issue where hundreds or thousands of antennas are installed at the base station to serve the group of users and minimize the interference. The mmWave band is emerged as most favorable band for massive MIMO.

\subsection{Benefits of MU- Massive MIMO}

MU-massive MIMO has attained a lot of attention for coming $5 \mathrm{G}$ wireless systems because of its high data rates for all the users and high reliability [13]. The multiple antennas that are installed at transmitter or receiver support multi signal paths to get large throughput. By adopting MU-massive MIMO technique the system capacity can be improved by factor of 10 and spectral efficiency by factor of 20 when the numbers of antennas installed at transmitter and receiver are about 256 [3]. Many more benefits can be achieved which are as follows:

i. Energy efficiency (EE) in modern cellular communications have growing attention worldwide. New research approach relative to EE called green cellular system has driven the mobile operators to meet the requirements of clean environment, decrease the cost and find solutions to improve the EE for cellular mobiles [14].

ii. In MU-Massive MIMO, we can reduce the numbers of amplifiers that uses a huge energy by replacing the traditional power amplifiers by a hundreds of low-cost power amplifiers [13], [15].

iii. Massive MIMO has a huge overflow of degree of freedom. Suppose we have 128 antennas that serve 28 users, then 100 degree of freedom remained unused [16].

\subsection{Estimation of Channel}

Channel state information (CSI) is important for improving the gain of multi-antenna in MIMO system. Therefore, CSI becomes more complicated for massive MIMO system because the huge numbers of antenna at base station. In addition, massive MIMO either applying FDD or TDD requires a large numbers of pilots. In the presence of large numbers of users, the reuse of same set of orthogonal pilots in the neighboring cells can result the pilot contamination in multi-cell system [17]. The pilot contamination limits the performance and decays the capacity of massive MIMO system. When the number of antennas are increased at base station, the SINR at receiver side will increase too. Because the number of antennas at base station are equal to the number of pilot contaminations, the data signals for all users decreased. To solve this problem, the SIC is applied to mitigate the interference between the pilot and data signals [18]. 


\subsection{Communications in Millimeter Waves}

Currently, all of the mobile system utilize spectrum in the range of $300 \mathrm{MHz}$ to $30 \mathrm{GHz}$. The advancement in electronic devices fabrication allows us to utilize the mmWave spectrum having large bandwidth for fifth generation $(5 \mathrm{G})$ wireless system networks. Therefore, the $5 \mathrm{G}$ wireless system must test the 30 to $300 \mathrm{GHz}$ spectrum for the broadband communication system. However, the issues and challenges in the use of mmWave spectrum need to be addressed [19]. Availability of huge bandwidth in mmWave and provision of a hundreds and thousands of antennas at base station make mmWave and massive MIMO promising candidates for (5G) [1] [20].

The high-path loss due to penetration and atmospheric attenuation become the nightmare for the mmWave. Many researches have investigated it and advised to adopt small cell system networks such as femtocell, microcell to overcome the high-path loss [21]. One of the most suitable solutions to avoid the high atmospheric attenuation transported by mmWave is to utilize huge numbers of antennas to the base station. In past and current wireless systems omnidirectional antenna array are used. The deployment of massive set of narrow-beam directional antennas can steer the signal direct to the multiple users. There are a lot of antenna array techniques but the most two fundamental techniques are the beamforming and spatial multiplexing. In spatial multiplexing, multiple separately and independent encoded data streams can be transmitted by using multiple antenna elements and the receivers have ability to reconstructing the original signal effectively [13, 22-25]. Spatial multiplexing is more effective in two cases:

i. When the path of prorogation is rich, it can support multiple data streams transmission.

ii. At high channel SNR the strength of the original signal is not be affected when it splits in to multiple data streams.

In beamforming approach, the base station adapts the weight of for each array of antenna elements to control the data streams direction [13]. In this way, the data stream targets the desired user and not interfere the others. Beamforming is suitable in the conditions bellow:

i. When the SNR of the channel is low.

ii. When the power of the channel is limited.

iii. Beamforming is critical for mmWave to avoid the high-path loss due to the small wavelength.

\section{SYSTEM MODEL}

We consider downlink multiuser channel for hybrid and digital precoding, where one base station (BS) serves $M$ users in the cell. BS has $N_{t}$ transmit antennas and each user equipped with $N_{r}$ receive antennas. Number of streams $\ell$ for each user are limited by $\ell \leq N_{r}$. Total number of RF chains $N_{r f}$ at transmitter and total number of streams $M \times \ell$ in the downlink are limited by $\min \left(N_{t}, M \times N_{r}\right)$ and $\min \left(N_{r f}, M \times N_{r}\right)$ respectively. The $\mathrm{RF}$ chains at receiver are assumed equal to receiver antennas. The signal received at the $m^{\text {th }}$ user is given by: 


$$
y=H_{m}^{M} W_{m} F_{m} \sqrt{P_{m} d_{m}}+\sum_{j \neq m}^{M} H_{m}^{M} W_{j} F_{j} \sqrt{P_{j} d_{j}}+n_{m}
$$

Where $n_{m}$ is vector of i.i.d. $C N\left(0, \sigma^{2}\right)$ noise and $P_{m}$ power allocated to the $m^{\text {th }}$ user.

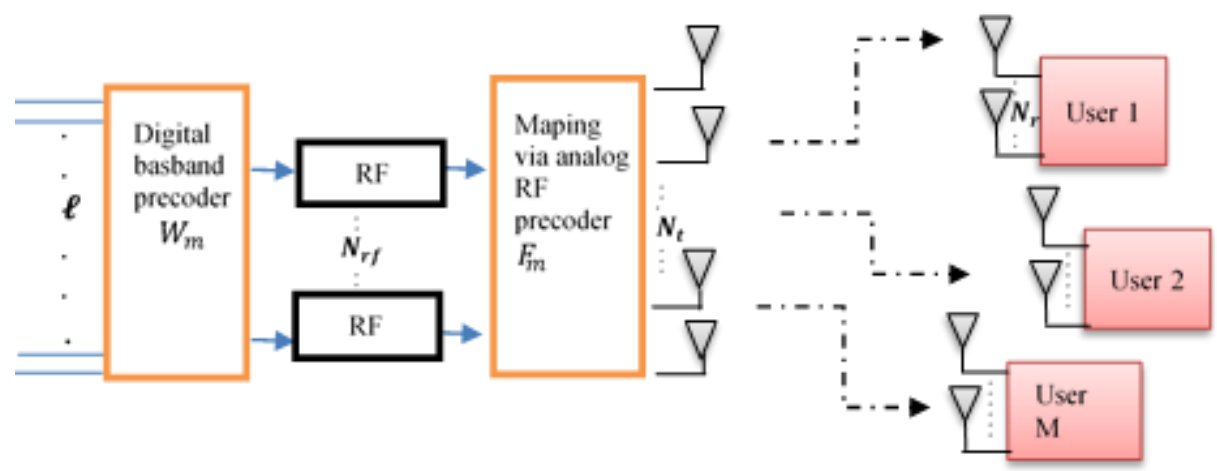

Figure 1. Proposed MU-Massive MIMO System.

\subsection{System Model for Digital Downlink Massive MIMO}

In digital downlink massive MIMO system, the BS uses precoding matrix $W=\left[W_{1}^{D}, W_{2}^{D}, \ldots \ldots ., W_{M}^{D}\right]$ at the transmitter where $W_{m}^{D} \in \square^{N_{t} \times \ell}$ is the matrix for $m^{t h}$ user. A linear matrix $G_{m}^{D} \in \square^{N_{r} \times \ell}$ may be used at the $m^{\text {th }}$ user to recover its symbols $d_{m}$ as:

$$
d_{m}^{D}=G_{m}^{D}\left(H_{m}^{H} W_{m} d+n_{m}\right)=G_{m}^{D}\left(H_{m}^{H} \sum_{i}^{M} W_{i} d_{i}+n_{m}\right)
$$

Where $H_{m}^{H} \in \square^{N_{t} \times N_{r}}$ is the massive MIMO channel between the $m^{\text {th }}$ receiver.

\subsection{System Model for Hybrid Downlink Massive MIMO}

Hybrid beamforming combines analog and digital precoders and decoders. If we expand the beamforming for multiuser massive MIMO, the estimated signal at $m^{\text {th }}$ user can written as:

$d_{m}^{H y}=G_{m}^{H y} R_{m}\left(H_{m}^{H} \sum_{i}^{M} F_{i} W_{i}^{H y} d_{i}+n_{m}\right)$

Where $W_{m}^{H y} \in \square^{N_{r f} \times \ell}, F_{m} \in \square^{N_{t} \times N_{r f}} \quad R_{m} \in \square^{N_{r} \times N_{r}}$ and $G_{m}^{H y} \in \square^{N_{r} \times \ell}$ are the matrices of baseband and analog precoding, and analog and digital decoding respectively. Every element of $F_{m} / R_{m}$ got a constant modulus. For simplicity, we denote $W_{m}^{H y}$ and $G_{m}^{H y}$ as $W_{m}$ and $G_{m}$ for further derivations. If we denote $A_{m}=G_{m} R_{m} H_{m}^{H} F_{m} W_{m}$ (Combining precoding and decoding of both digital and analog), then the received SNR at $m^{\text {th }}$ user is:

$$
\gamma_{m, t}=\frac{\left\|A_{m}(t, t)\right\|^{2} P_{m}}{\sum_{i \neq u}^{\ell}\left\|A_{m}(t, t)\right\|^{2} Q_{m}+\sum_{j \neq m}^{M}\left\|A_{m} A^{j}(t, t)\right\|^{2} P_{j}+\sigma^{2}}
$$




\subsection{Channel Model}

There are high free-space path losses in mmWave propagation environment that must be characterized by an appropriate channel model. We adopt cluster channel, described in [11]. This model performs the mmWave channel matrix as:

$$
H=\sqrt{\frac{N_{t} N_{r}}{N_{c l} N_{\text {ray }}}} \sum_{i=1}^{N_{c l}} \sum_{l=1}^{N_{r a y}} \alpha_{i l} a_{r}\left(\phi_{i l}^{r} \theta_{i l}^{r}\right) a_{t}\left(\phi_{i l}^{t} \theta_{i l}^{t}\right)^{H}
$$

Where $N_{c l}$ is the numbers of clusters, $N_{\text {ray }}$ the numbers of rays for each cluster and the gain of $l^{\text {th }}$ ray in the $i^{\text {th }}$ prorogation channel is representing by $\alpha_{i l}$.

\section{PROBLEM FORMULATION AND HYBRID PRECODING DESIGN}

We want maximize the total sumrate of the system, which is given as:

$\arg _{W_{m} F_{m} R_{m} G_{m}} \max \sum_{m}^{M} \sum_{t=u}^{\ell} \log \left|1=\gamma_{m, t}\right|$

s.t. $\sum_{m}^{M}\left\|W_{m} F_{m}\right\| \leq M \ell$

The above problem is non-convex and it is difficult to obtain its optimal solution. Therefore, we propose to solve an approximation of (6) in order to find near-optimal solution that can be practically implemented. First, we fix analog precoder and define effective channel matrix

$$
H_{e f f}=H_{m}^{M} F_{m}
$$

We take SVD of effective channel for digital precoder and decoder design.

$$
H_{\text {eff }}=U_{\text {eff }} A_{\text {eff }} V_{\text {eff }}^{*}
$$

$$
W_{m}=V_{\text {eff }} \quad \text { and } F_{m}=U_{e f f}^{*}
$$

We design the hybrid precoding in two steps, starting with analog and digital decoders and then analog and digital precoder by the help of orthogonal algorithm. As we can note from equation (3) the matrices of hybrid are more constrained than the matrices of digital beamforming, which means digital beamforming achieves the best performance.

Due to the advantages of block diagonalization, we employ the $d_{m}^{D}$ as the received reference signal. We set the characteristics of proposed hybrid beamforming by finding the Euclidian distance between the received signal for digital and hybrid beamforming. It can be acquired by estimating the mean square error between the $d_{m}^{D}$ and $d_{m}^{H y}$ that is given by [1]:

$$
\xi_{m}=\mathrm{E}\left[\left(d_{m}^{D}-d_{m}^{H y}\right)\left(d_{m}^{D}-d_{m}^{H y}\right)^{H}\right]
$$

We use the block diagonalization digital beamforming for total sum rate maximization of multiuser massive of downlink channel. The block diagonalization uses three basic steps as follows:

i- $\quad$ Firstly, it eliminates the interference of other users

ii- Secondly it cancels the self-interference for each desired user. 
iii- It optimizes the power of each symbol for maximizing the total sum rate. For better clarification of the above steps we can define these as follows:

$$
\begin{aligned}
& \hat{H}_{m} \square\left[H_{1}, H_{2}, \ldots \ldots H_{m-1}, H_{m+1}, \ldots H_{M}\right] \\
& V_{h 0 m} \square \operatorname{null}\left(\hat{H}_{m}^{H}\right) \\
& X_{m} \square H_{m}^{H} V_{h 0 m}=\hat{U}_{m h} Z_{m} \hat{V}_{h m}^{H}
\end{aligned}
$$

Where $V_{h 0 m} \in \square^{N_{t} \times \ell}$ is the first factor of $\ell$ of $\operatorname{null}\left(\hat{H}_{m}^{H}\right), \quad \hat{U}_{m h} \in \square^{N_{r} \times \ell}, \hat{V}_{h m}^{H} \in \square^{\ell \times \ell}$ are unitary matrices and $Z_{m}$ is the diagonal matrix of size $\ell$. As we mention above the BD beamforming algorithm can be carry out by putting $W_{m}$ and $G_{m}$ of (2) as $W_{m}=V_{h 0 m} \hat{V}_{\mathrm{h} 0 m}^{H} \sqrt{Q_{m}}$ and $G_{m}=\hat{U}_{h m}$, the $Q_{m}$ is denoted the matrix of power allocation for each user. Therefore, the relation between the input-output in (2), can be rewritten as:

$\hat{d}_{m}^{D}=Z_{m} \sqrt{Q_{m}} d_{m}+\hat{U}_{m h}^{H} n_{m} \Rightarrow \hat{d}_{m i}^{D}=z_{m i} \sqrt{q}_{m i} d_{m}+\hat{u}_{m h i}^{H} n_{m}$

Where the $\hat{d}_{m i}^{D}$ denoted the elements of ${ }^{\hat{d}_{m}^{D}}$ and the diagonal element is represented by $z_{m i}\left(q_{m i}\right)$ of $Z_{m} Q_{m}$ and finally, the $\hat{u}_{m h i}^{H}$ denoted the row of $\hat{U}_{m h}^{H}$.

The final steps of BD can be performed by solving the total sum rate maximization as:

$\max \left(q_{m i}\right)=\sum_{m=1}^{M} \sum_{i=1}^{\ell} \log _{2}\left(1+\frac{z_{m i}^{2} q_{m i}}{\sigma^{2}}\right)$

s.t. $\sum_{m=1}^{M} \sum_{i=1}^{\ell} q_{m i}=P_{\max }$

The elements of maximum power at transmitter $P_{\max }$ is represented by the $q_{m i}$. The aim is to solve this problem by utilize some simple algorithms such as water filling algorithm to reach the close-optimal solution.

Form the above equations we can recognize that, the BD beamforming $R_{m m}=Z_{m} \sqrt{Q_{m}}$ and $R_{m i}=0, \forall m \neq i$. Therefore, the $\xi_{m}$ in (10) can be written as:

$$
\begin{aligned}
& \xi_{m}=\left(\hat{G}_{m}^{H} R_{m}^{H} H_{m}^{H} F_{m} \hat{W}_{m}-Z_{m} \sqrt{Q_{m}}\right)-\left(\hat{G}_{m}^{H} R_{m}^{H} H_{m}^{H} F_{m} \hat{W}_{m}-Z_{m} \sqrt{Q_{m}}\right)^{H}+ \\
& \sum_{i=1, i \neq m}^{M}\left(\hat{G}_{m}^{H} R_{m}^{H} H_{m}^{H} F_{m} \hat{W}_{i}\right)\left(\hat{G}_{m}^{H} R_{m}^{H} H_{m}^{H} F_{m} \hat{W}_{m}\right)^{H}+\sigma^{2}\left(\hat{G}_{m}^{H} R_{m}^{H}-\hat{U}_{h m}\right)\left(\hat{G}_{m}^{H} R_{m}^{H}-\hat{U}_{h m}\right)^{H}
\end{aligned}
$$

Now we have to design analog precoder $F_{m}$, digital precoder $\hat{W}_{m}$ and analog decoder $R_{m}$ . The gap between digital and hybrid beamforming is constant for all the symbols. We use WSMSE algorithm to optimize the main objective function i.e. the $i^{\text {th }}$ symbol weight of the $m^{\text {th }}$ user is set to $\frac{1}{z_{m i}^{2} q_{m i}}$, and the equation can be mathematically solved as:

$$
\min _{F_{m} \hat{W}_{m} R_{m}} \sum_{m=1}^{M} \operatorname{tr}\left\{\left(z_{m} \sqrt{Q_{m}}\right)\right\}^{-1} \xi_{m}\left\{\left(z_{m} \sqrt{Q_{m}}\right)\right\} \square \xi_{m}
$$


s.t. $\quad \sum_{m=1}^{M} \operatorname{tr}\left\{F_{m} \hat{W}_{m} \hat{W}_{m}^{H} F_{m}^{H}\right\}=Q_{\max }$

$$
\left|F_{m(i, j)}\right|^{2}=M, \quad\left|R_{m(i, j)}\right|^{2}=M
$$

The $\sum_{m=1}^{M} \operatorname{tr}\left\{F_{m} \hat{W}_{m} \hat{W}_{m}^{H} F_{m}^{H}\right\}=Q_{\text {max }}$ is introduced to make the same total power for hybrid and digital beamforming. We further optimize the problem in two steps:

i. First optimize the receiving matrix (digital decoder) and analog decoder for each user.

ii. Then optimized analog and digital precoders for fixed analog and digital decoders.

\subsection{First Step}

In the first step, we optimized jointly analog $R_{m}$ and digital $G_{m}$ decoders for $m^{\text {th }}$ user, we written it as:

$\min _{\overline{\bar{W}}_{m} F_{m}}\left|\overline{\bar{W}}_{m} F_{m}-\overline{\bar{U}}_{h m}\right|_{F}^{2}$, s.t. $\left|F_{m}\right|^{2}=1$

As we observed that $\overline{\bar{W}}_{m}$ and $F_{m}$ are joint function. Therefore, the constraint of the objective function is non-convex and optimal solution of objective function is difficult to find. To solve function in (17) we set the variable of $\bar{F}_{m}=\mathbf{f}_{m}$ where the $\mathbf{f}_{m}=\left[F_{m} n u l l\left(\bar{F}_{m}^{T}\right)\right]$ and rewrite the (17) as:

$$
\min _{G_{m}}\left|\bar{R}_{m} \bar{G}_{m}-\overline{\bar{U}}_{h m}\right|_{F}^{2}, \quad \text { s.t. }\left\|\operatorname{diag}\left(\bar{R}_{m} \bar{R}_{m}^{H}\right)\right\|_{0}=N_{r f}
$$

But even after some mathematical manipulation the non-convex problem is still the same. The better solution to overcome non-convex problem is to use the orthogonal matching pursuit algorithm as given below: 


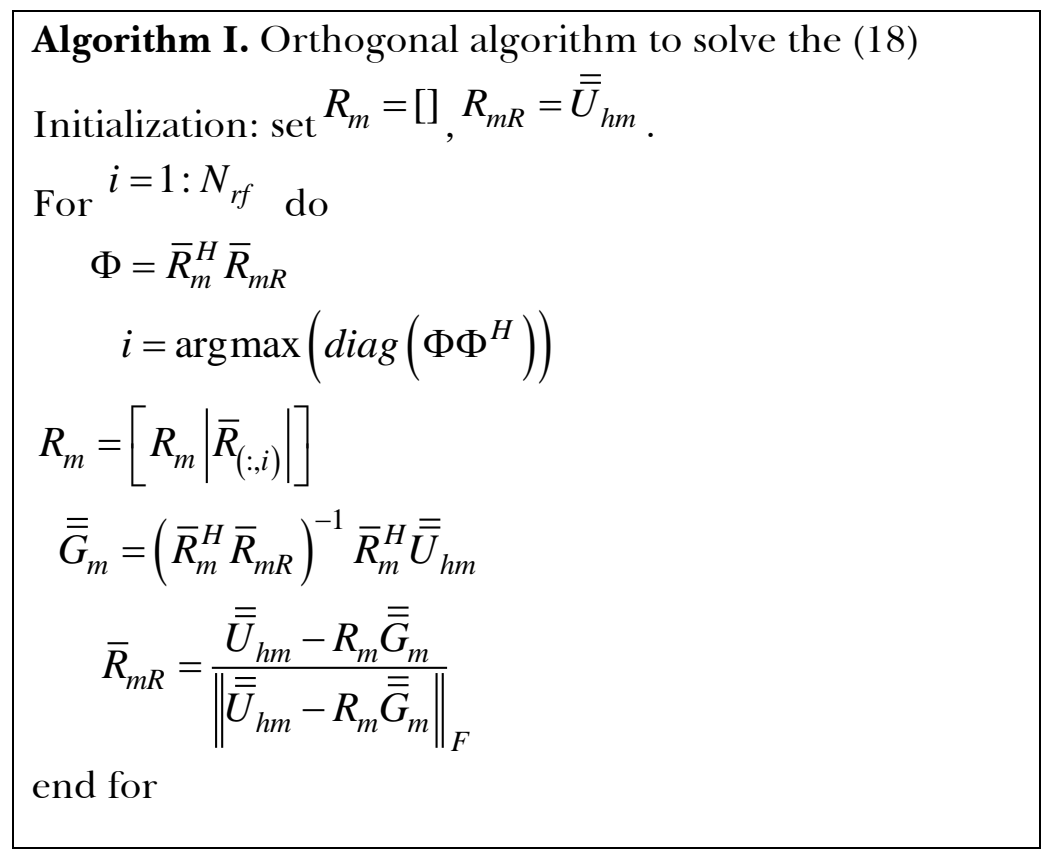

\subsection{Second Step}

For a given $\overline{\bar{G}}_{m}$ and $\overline{\bar{R}}_{m}$ in (18), the problem can be reformulated as:

$$
\begin{gathered}
\max _{F_{m} \hat{W}_{m}}\left\|\overline{\bar{G}}_{m} R_{m}^{H} H_{m}^{H} F_{m} \bar{W}_{m}-I\right\|_{F}^{2}, \\
\text { s.t. } \operatorname{tr}\left\{F_{m} W_{m} W_{m}^{H}\right\} \leq P_{\max } \\
\left|F_{(i, j)}\right|^{2}=1
\end{gathered}
$$

We can notice form (15) that the correlation of analog precoder is much high with right singular value of $H_{m}^{H}$. It is assumed that each column of analog precoder $F_{m} \mathbf{F}_{\mathbf{m}}$ is derived from one of the column of $\mathbf{f}_{m}$, where $\mathbf{f}_{m}=\left[F_{m} \operatorname{null}\left(\bar{F}_{m}^{T}\right)\right] \in \square^{N_{t} \times N_{t}}$ with normalized entries. Hence the modulus value of analog precoder $\mathbf{f}_{m}$ is set to 1 . By setting $\bar{F}_{m}=\mathbf{f}_{m}$ and applying the same technique as step1, the problem (19) can be rearrange as

$$
\max _{\bar{F}_{m}}\left\|\left(R_{m} H_{m} \overline{\bar{G}}_{m}\right)^{H} \bar{F}_{m} \bar{W}_{m}-I\right\|_{F}^{2}
$$

s.t. $\operatorname{tr}\left\{\bar{F}_{m} \bar{W}_{m} \bar{W}_{m}^{H} F_{m}^{H}\right\}=P_{\max }$

$\left\|\operatorname{diag} \bar{W}_{m} \bar{W}_{m}^{H}\right\|=N_{r f}$

Therefore, the solution to this problem can be found using the orthogonal algorithm as used for (18) in step 1, as follows: 


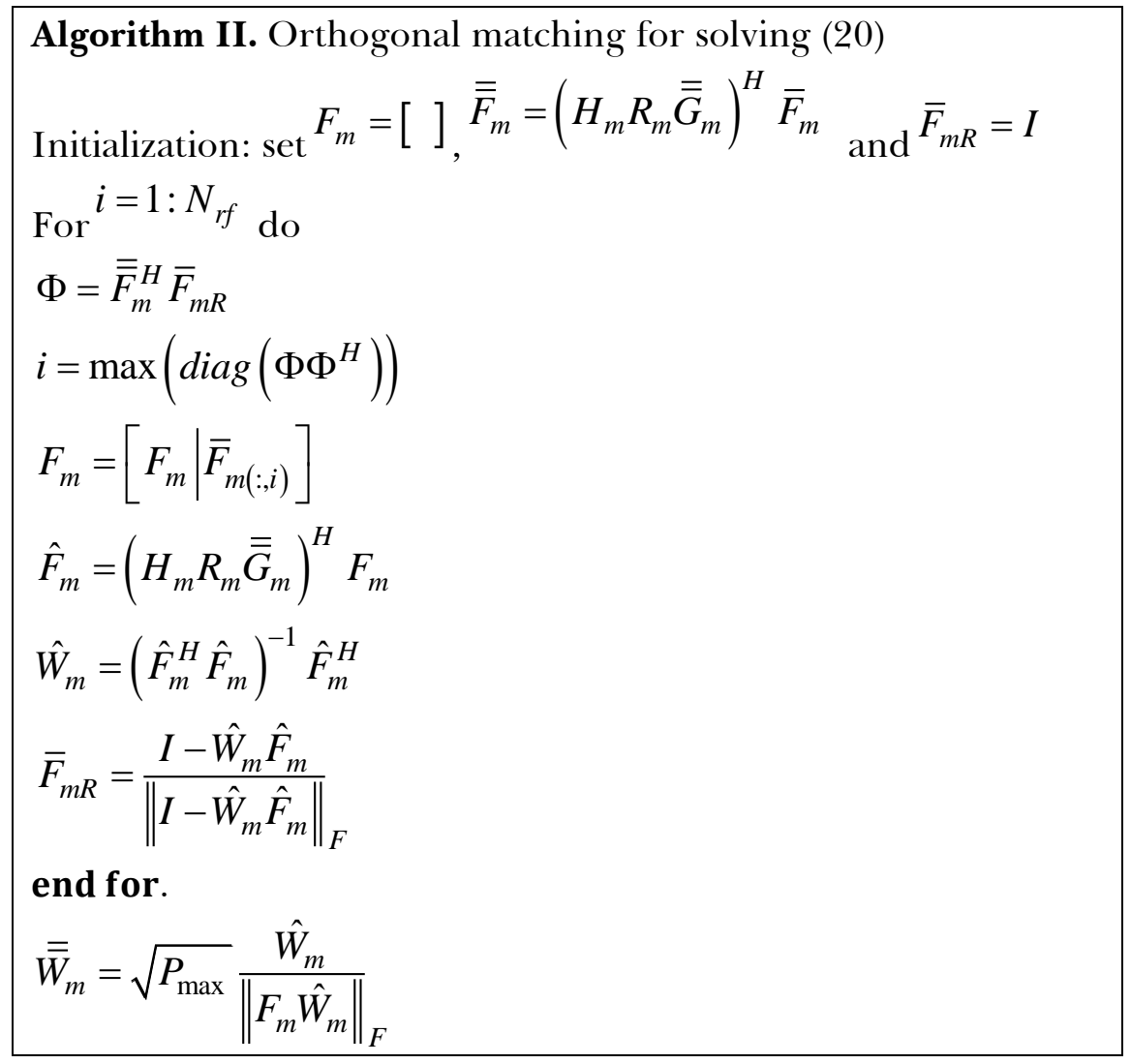

It is mentioned that the step 1 and step 2 both use fix the power at transmitter side.

\section{SIMULATION RESULTS}

MIMO This section presents the simulation results. We use, $N_{t}=128, N_{r}=32$, where is $N_{t}$ is the number of antenna at BS and $N_{r}$ is the number of antennas at each user's equipment. The number of users are $M=4$, antenna spacing $d=0.5 \lambda$, the number of paths are $L=16$. The $S N R=\frac{P_{a v}}{\sigma^{2}}$ is dominated by $\sigma^{2}$ where $P_{a v}=\frac{P_{\max }}{\ell}$ and the total power is limited by $P_{\max }=M \ell$. The channel parameters

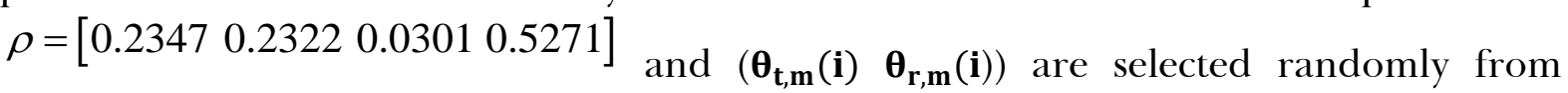
uniform distribution in $[0,2 \pi]$. Then the computed sum total rate is given as $R_{t}=\sum_{m=1}^{M} \sum_{i=1}^{\ell} R_{m i}$, which means the sum total rate for each symbol is computed by $R_{m i}=\log _{10}\left(1+\gamma_{m i}\right)$, where $R_{m i}$ is the achieved SINR of $m^{\text {th }}$ user $i^{\text {th }}$ stream. Each plot is generated by 1000 iterations, $\forall M$.

\subsection{Compression between Hybrid and Digital Beamforming}

In this part, we compare the performance of hybrid beamforming with the digital beamforming. For simulation, we set the data streams $\ell$ to 12 and the number of radio frequency chains $N_{\text {rf }}$ to 16 . The achievable rates for the digital and hybrid beamforming are shown in Figure 2 It can be observed that hybrid beamforming performance 
approaches the digital beamformig performance from low to medium SNR region. In addition, it can be seen the small gap performance may occur at high SNR region.

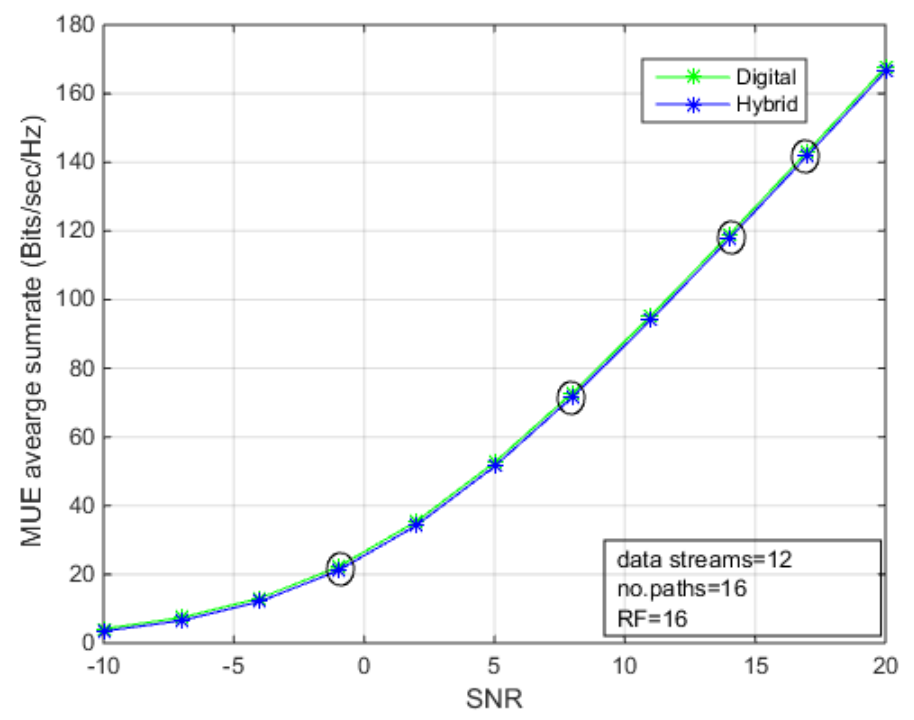

Figure 2. Digital and Hybrid Beamforming Compression.

\subsection{J oint Effects of $\ell$ and $N_{r f}$ On Hybrid and Digital Beamforming}

In this section we study the joint effect of data streams and radio frequency chains $N_{r f}$ on both hybrid and digital beamforming performance. We set $\mathrm{SNR}=5 \mathrm{~dB}$. Figure 3 shows the performance comparison of both schemes. It can be noted that by increasing the data streams $\ell$, the achievable sum rate of hybrid and digital beamforming will also increase. The data streams are limited by the constant of maximum power $P_{\max }=M \ell$. By decreasing the data streams, the performance gap between digital and hybrid can be bridged. If we limit the number of radio frequency chain $N_{r f}$ and the analog to digital converters (ADCs), the performance gab between both schemes digital and hybrid will decrease by reducing the number of streams $\ell$.

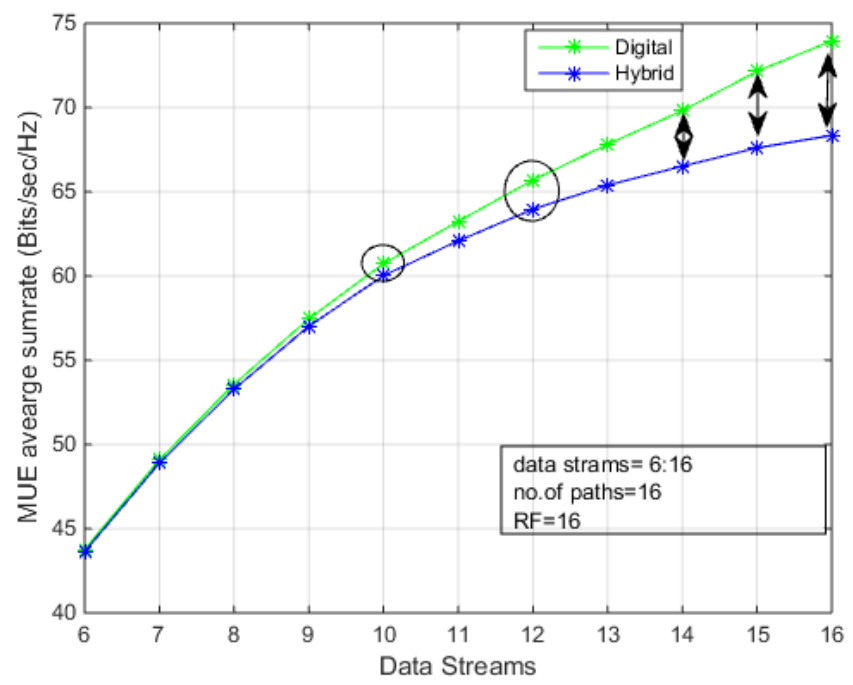

Figure 3. Joint Effects Of $\ell$ and $N_{r f}$ On Digital and Hybrid Beamforming. 


\subsection{Effect of ${ }^{N_{r f}}$ on hybrid beamforming}

In this part, the performance of the hybrid beamforming is examined by varying the number of RF chains. Figure 4 presents of hybrid algorithm for deferent setting of $N_{r f}=8: 20$, at $\mathrm{SNR}=5 \mathrm{~dB}$ and $\ell=8$. We can see that by increasing the number of RF chains and ADCs, the performance of the hybrid algorithm can be improved significantly and the gap between the two schemes becomes small and almost constant when the number of RF chains are more than the twice of the data streams.

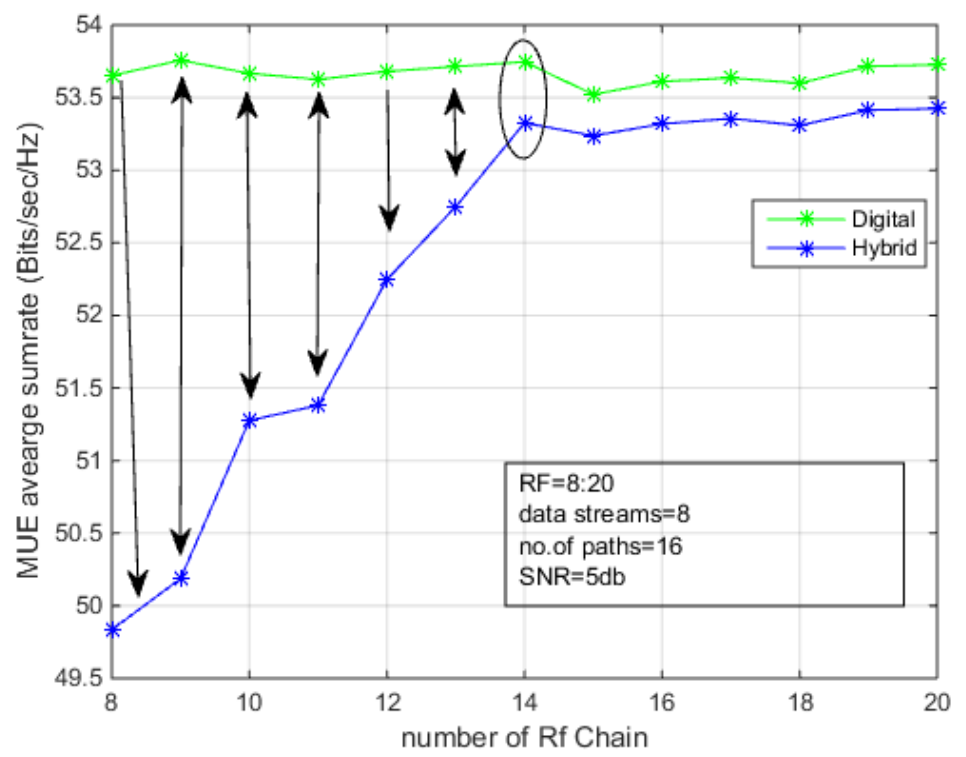

Figure 4. Effect of $N_{r f}$ on the Hybrid Beamforming.

\section{CONCLUSION}

We have designed a hybrid beamforming scheme for downlink MU-massive MIMO system. Hybrid scheme is indirectly designed by using WSMSE to maximize the total sumrate, whereas conventional block diagonalization is used for digital beamforming. We examine the relation between two by considering different parameters such as SNR, number of data symbols and the number of RF chains and ADCs. From the result we conclude that the performance gap between hybrid and digital beamforming can be bridged by decreasing the number of data streams for a given number of ADCs and RF chains. On the other hand, when the number of data streams are fixed, an increase in $\mathrm{RF}$ chains and $\mathrm{ADC}$ increases the total sum rate of hybrid scheme that we expect. The hybrid beamforming reaches the same performance of digital beamforming when SNR reaches from the low to the high region. 


\section{REFERENCES}

[1] T. E. Bogale and L. B. Le, "Beamforming for Multiuser Massive MIMO Systems: Digital Versus Hybrid Analog-Digital," Proceedings of the IEEE Global Communications Conference, Austin, USA, (2014) December 8-12.

[2] Z. Xinying, A. F. Molisch, and K. Sun-Yuan, "Variable-Phase-Shift-Based RFBaseband Codesign for MIMO Antenna Selection," IEEE Transactions on Signal Processing., vol. 53, no. 11, (2005), pp. 4091-4103.

[3] Y. Gao, M. Khaliel, and T. Kaiser, "Wideband Hybrid Analog-Digital Beamforming Massive MIMO Systems Based on Rotman Lens," Proceedings of the IEEE International Conference on Communication Systems (ICCS), Shenzhen, China, (2016) December 14-16.

[4] O. El Ayach, R. W. Heath, S. Abu-Surra, S. Rajagopal, and Z. Pi, " Low Complexity Precoding for Large Millimeter Wave MIMO Systems," Proceedings of the IEEE International Conference on Communications (ICC), Ottawa, Canada, (2012) June $10-15$.

[5] E. Zhang and C. Huang, "On Achieving Optimal Rate of Digital Precoder by RFBaseband Codesign for MIMO Systems," Proceedings of the IEEE 80th Vehicular Technology Conference (VTC2014-Fall), Vancouver, Canada, (2014) September 14-17.

[6] S. He, C. Qi, Y. Wu, and Y. Huang, "Energy-Efficient Transceiver Design for Hybrid Sub-Array Architecture MIMO Systems," IEEE Access., vol. 4, pp. 98959905.

[7] A. Li and C. Masouros, "Hybrid Analog-Digital Millimeter-Wave MU-MIMO Transmission with Virtual Path Selection," IEEE Communications Letters., vol. 21, no. 2, (2017), pp. 438-441.

[8] S. Park, A. Alkhateeb, and R. W. Heath, "Dynamic Subarrays for Hybrid Precoding in Wideband mmWave MIMO Systems," IEEE Transactions on Wireless Communications., vol. 16, no. 5, (2017), pp. 2907-2920.

[9] R. Rajashekar and L. Hanzo, "Hybrid Beamforming in mm-Wave MIMO Systems Having a Finite Input Alphabet," IEEE Transactions on Communications., vol. 64, no. 8, (2016), pp. 3337-3349.

[10] D. Vouyioukas, "A Survey on Beamforming Techniques for Wireless MIMO Relay Networks," International Journal of Antennas and Propagation., (2013), pp.1- 21.

[11] Z. Pi and F. Khan, "An Introduction to Millimeter-Wave Mobile Broadband Systems," IEEE Communications Magazine., vol. 49, no. 6, (2011), pp. 101-107.

[12] M. K. Samimi and T. S. Rappaport, "3-D Statistical Channel Model for MillimeterWave Outdoor Mobile Broadband Communications," Proceedings of the IEEE International Conference on Communications (ICC), London, UK, (2015) June 812.

[13] J. Wu, Y. Zhang, M. Zukerman, and E. K. N. Yung, "Energy-Efficient Base-Stations Sleep-Mode Techniques in Green Cellular Networks: A Survey," IEEE Communications Surveys \& Tutorials., vol. 17, no.2, (2015), pp. 803-826. 
[14] S. Han, C. 1. I, Z. Xu, and C. Rowell, "Large-Scale Antenna Systems With Hybrid Analog and Digital Beamforming for Millimeter Wave 5G," IEEE Communications Magazine, vol. 53, no.1, (2015), pp. 186-194.

[15] L. Dai, X. Gao, J. Quan, S. Han, and I. C. L, "Near-Optimal Hybrid Analog and Digital Precoding for Downlink mmWave Massive MIMO Systems," Proceedings of the IEEE International Conference on Communications (ICC), London, UK, (2015) June 8-12.

[16] A. Osseiran, F. Boccardi, V. Braun, K. Kusume, P. Marsch, M. Maternia. O. Queseth, M. Schellmann, H. Schotten, H. Taoka and H. Tullberg, "Scenarios for 5G Mobile and Wireless Communications: The Vision of the METIS Project," IEEE Communications Magazine., vol. 52, no. 5, (2014), pp. 26-35, 2014.

[17] X. Yu, J. C. Shen, J. Zhang, and K. B. Letaief, "Alternating Minimization Algorithms for Hybrid Precoding in Millimeter Wave MIMO Systems," IEEE Journal of Selected Topics in Signal Processing., vol. 10, no, 3, (2016), pp. 485-500.

[18] A. Alkhateeb, O. E. Ayach, G. Leus, and R. W. Heath, "Channel Estimation and Hybrid Precoding for Millimeter Wave Cellular Systems," IEEE Journal of Selected Topics in Signal Processing., vol. 8, no, 5, (2014), pp. 831-846.

[19] O. E. Ayach, S. Rajagopal, S. Abu-Surra, Z. Pi, and R. W. Heath, "Spatially Sparse Precoding in Millimeter Wave MIMO Systems," IEEE Transactions on Wireless Communications., vol. 13, no, 3, (2014), pp. 1499-1513.

[20] M. N. Khan and M. Jamil, "Performance Improvement in Lifetime and Throughput of LEACH Protocol," Indian Journal of Science and Technology, vol. 9, no. 21, (2016), pp. 1-6.

[21] M. N. Khan and Jamil, "Maximizing Throughput of Free Space Communication Systems Using Puncturing Technique," Arabian Journal for Science and Engineering, vol. 39, no. 12, (2014), pp. 8925-8933.

[22] M. N. Khan, H. K. Hasnain and M. Jamil, "Digital Signal Processing: A Breadthfirst Approach”, Rivers Publishers, Denmark, (2016), pp. 221-247.

[23] J. G. Andrews, S. Buzzi, W. Choi, S. V. Hanly, A. Lozano, A. C. K. Soong, et al., "What Will 5G Be?," IEEE Journal on Selected Areas in Communications., vol. 32, no, 6, (2014), pp. 1065-1082.

[24] C. Li, Y. Ying, C. Xiaohui, and W. Weidong, "Multi-Stage Beamforming Codebook for $60 \mathrm{GHz}$ WPAN," Proceedings of the 6th International ICST Conference on Communications and Networking in China (CHINACOM), Harbin, China, (2011) August 17-19.

[25] M. H. Alsharif and R. Nordin, "Evolution towards Fifth Generation (5G) Wireless Networks: Current Trends and Challenges in the Deployment of Millimetre Wave, Massive MIMO, and Small Cells," Telecommunication Systems., vol. 64, no. 4, (2017), pp. 617-637. 


\section{A REVIEW ON ANTECEDENTS OF CITIZEN'S TRUST IN GOVERNMENT SOCIAL MEDIA SERVICES}

Sohrab Khan

Advanced Informatics School, Universiti Teknologi Malaysia, Kuala Lumpur, (Malaysia)

E-mail: meersohrab@gmail.com

Nor Zairah Ab. Rahim

Advanced Informatics School, Universiti Teknologi Malaysia, Kuala Lumpur, (Malaysia)

E-mail: nzairah@utm.my

Nurazean Maarop

Advanced Informatics School, Universiti Teknologi Malaysia, Kuala Lumpur,

(Malaysia)

E-mail: nurazeanmaarop@gmail.com 


\section{ABSTRACT}

In recent years, social media has been accepted as a practice of e-government services across the world. Unlike static government websites, social media is an interactive communication platform that helps government organizations to improve their relationship with citizens through participation and engagement. However potential values of social media cannot be displayed without addressing citizen's acceptance and their trust to participate in government social media services. Majority of previous studies on the relationship of trust between government and public are conducted on static government websites using general technological characteristics. This paper provides a comprehensive literature review of the relevant articles to explore multiple antecedents or factors of citizen's trust with a major consideration in using social media as a technology platform for e-government services. A model is proposed that identifies individual characteristics, government factors, risk factors and social media characteristics as multiple antecedents of citizen's trust in government social media services.

\section{KEYWORDS}

Antecedents of trust; e-government; government social media; citizen's trust, factors.

\section{INTRODUCTION}

E-government is the employment of information and communication technologies (ICTs) by government to provide services to citizens, business partners and different government offices [1]. The goal of e-government is to enrich government online services and their quality of interactions with citizens and businesses partners through good quality services and systems. According to Khan [2] investment in e-government, also known as Government 1.0, has enabled government organizations to be more transparent, effective, and efficient than early traditional service delivery mechanisms.

In recent years the development of e-government services has migrated from static websites to social media, which is considered as part of Web 2.0 platform [3]. As stated by Hao, Zheng and Zeng [4], the benefits of social media are not limited only to individuals and private sectors organizations. Social media also provides unique opportunities for government organizations to make effective use of it to provide services to citizen's as per their desire and needs. Government organizations in different countries have started using social media to build closer ties with citizens and increase their participation in government services [5,6]. The statistics show that the usage of social media by governments across the world is increasing rapidly with the number more than three times from 2010 to 2012 and with another 50 per cent rise in 2014 [7]. Social media is regarded as an influential tool to activate citizen-government engagement. It facilitates online communication and relationship building between government and citizens through information sharing and non-discriminative participation [8]. Social media has the capability to increase legitimacy of government organizations through communication and coordination between multiple stakeholders. Government organizations can integrate the information obtained from citizens into policy making and thus increase transparency through information sharing and collaboration with public to reach solutions for government problems. The citizens at the same time can get the opportunity to communicate and express their opinions on government policies and quality of services in real time environment [6]. The government use of social media is supported to strengthen citizen's interactivity [9], increase government transparency [10] 
and perception of trustworthiness [11]. However, the efforts of governments and the potential value of government social media services cannot be displayed without addressing citizens willingness and their trust to participate in these services $[4,8]$.

Several studies have been conducted to investigate the relationship of trust between people and government [1, 12]. Bélanger and Carter [13] identified trust as a significant predictor of citizen's intention to use an e-government service. Similarly, Abu-Shanab [14], mentioned that the gate to e- government adoption is trust. This was supported by Chen, Jubilado, Capistrano and Yen [15] by highlighting trust as an essential determinants for e-government adoption. Though previous studies have reflected trust as the most significant dimension to influence citizens behavior towards e-government adoption. However, majority of those studies are limited on e-government websites using internet as a technology to provide e-government services. In addition, majority of them have addressed partial issues with limited consideration only about trust in internet and trust in government. Very little attention has been paid on citizen's trust perspective in online government services [12]. On the contrary there is very little research on using social media as a technology in government organizations[4]. Despite of increased popularity in using social media by governments across the world, its acceptance among citizen's is still a serious issue and has not gained much attention in previous studies [16]. Prior studies have identified trust as a significant factor in using social media or social networking sites at user level [17] and firm level [18] but have failed to cover multiple perspective of citizen's trust in context of using government social media services. Some studies have also investigated the relationship of social media with interactivity [4], participation [4] and to increase citizen's trust on institutions and their services [5,9]. However, the major contributors or factors of citizen's trust to participate in government social media services are still unexplored.

Trust is a major concern that develops citizens acceptance to participate with government and use its online services [19, 20]. Al-Khouri [21] mentioned about the need to develop citizen's trust if their acceptance and participation in e-government services are to be promoted. Therefore, this can be argued that citizen's trust on government social media services must be developed a way before they start using these services. It is important to explore the major contributors to develop citizen's trust to gain an understanding about their behavior towards government social media services. The objective of this paper is to identify those factors, which can influence citizen's trust to participate in government social media services. This paper contributes in literature by exploring antecedents of citizen's trust from multiple dimensions with a major focus in using social media as a technology for electronic government services. The identified factors are categorized, and an integrated model is proposed to provide a comprehensive understanding of different trust dimensions. The findings of this paper can help government organizations to better understand citizen's needs and design their policies accordingly to increase citizen's trust and participation in government social media services.

\section{METHODOLOGY}

A literature review was conducted on citizen's trust perspectives and their participation in e-government and social networking services. The search was carried out through seven electronic databases: that are ACM, Science Direct, IEEE, Scopus, Springer link, Taylors and Francis and Google Scholar. These databases were chosen as they provide 
the most important and high impact full-text journals and conference proceedings, covering the fields of e-government, social media, social networking sites and government social media services. The Keywords were chosen in respect of factors Influencing Citizens Trust which include: "Trust", "Citizens Trust", "E government", "Factors", "Government social media", "social networking services". The resulted articles were thoroughly analyzed to extract most relevant articles meeting the research needs of this paper to identify antecedents of trust in government social media. Initially 291 articles were identified through database searches. The duplicate studies, short papers and the articles related with e-government or social media implementation, challenges, strategies and showing government organizations perspective were not included in this paper. Thus, 32 articles were selected which were more relevant about trust factors influencing citizen's behavior. In addition, reference lists of selected articles were scanned to identify any further relevant articles that were not found in the initial search. As a result, total number of 36 articles were found relevant to identify trust factors in context of government social media services.

\section{THEORETICAL FOUNDATION AND RELATED STUDIES ON ANTECEDENTS OF TRUST}

The field of Information systems and Technology has always attracted researchers' interest to investigate the theories and models that have the power in predicting and explaining behavior. To provide a solid theoretical basis for selecting influential trust factors in government social media services, this paper integrates two important streams of literature. First is the Technology acceptance model (TAM) [22], which is an adaptation of Theory of reasoned action (TRA) [23] and secondly, the literature on multiple dimensions of citizen's trust [12-14, 24].

TAM is one of the most popular research model that was developed to predict behaviour in IT usage [3]. TAM seeks to conceptually understand and explain an individual's intention about IT system usage, based on two perceptions. The first is the user's perception regarding whether it will help his or her performance of relevant tasks. This is referred to as perceived usefulness (PU). The second perception is the user's perception regarding the effort required to use a system. This is referred to as perceived ease of use (PEOU). Both these perceptions about the technology are said to shape the intention to use, which in turn shapes the actual use of the technology. The value of TAM in context of e-government [12, 25] and social networks [3] has been consistently important. TAM is considered as most successful adoption model as per the number of studies on online behavior relying on TAM and its high explanatory power [26]. TAM as a standalone model might not sufficiently explain the intention and use of the technology but has the flexibility to be extended and adapted to different features by integrating additional constructs from other models and cognitive studies [26]. TAM is considered as one of the best frameworks to understand technology adoptions and this can be extended or adapted easily to different features in many diverse situations [26]. The connections between trust and TAM has been discussed in previous literature showing a significant relationship of PEOU (perceived ease of use) and PU (perceived usefulness) with trust in different contexts of online environments [27]. This paper contributes to integrate antecedents of trust with TAM in context of government social media services which is a new practice of e-government services. This paper will determine how multiple dimensions of citizen's trust integrated with TAM can affect citizen's intention to participate in government social media services. 
Trust refers to the willingness of one party to become vulnerable to others based on their characteristics [28, 29]. Trust has been studied in various disciplines including psychology, sociology, computer science, business and organizational sciences. In each field Trust, has been interpreted differently, which causes complexity in defining trust. Because of its multidimensional nature trust has attracted attention from multiple perspectives and thus there are various definitions of trust in literature [24]. The literature shows that majority of previous studies have viewed technology and government factors as important antecedents of trust in using online government services [30-32]. For instance, Wang and Lu [32] stated that trust in government and trust in internet are main factors that determine citizen trust on e-government. This was supported by Chen, et al. [15] in their study by identifying trust in technology and trust in government as essential factors influencing trust in e-government websites. There are very few studies that have addressed multiple perspectives of citizen's trust in using government services $[12,14]$. One of the study was conducted by Beldad, Geest, Jong and Steehouder [33] who categorized trust in online environment into three dimensions: which are Internet user based, Organization based and Web based determinants of trust in online environment. In a recent article Alzahrani, et al. [12] carried out a systematic literature review on citizens trust aspects and categorized them as technology factors, government factors, risk factors and citizen's characteristics. Beside technological and government factors they highlighted the consideration of risk related factors and psychological aspects of individuals in determining their trust towards e-government services. However, their study was limited on e-government websites considering general technological characteristics.

The context of this paper is on government social media services with a focus on social media characteristics instead of focusing on technology characteristics in general. In this study, social media is being used as a technology platform to provide government services, contrary to the previous studies where Internet was used as a tool to provide services from static government websites [12, 14, 15]. Based on the study of Alzahrani, et al. [12] the trust factors are categorized in to multiple dimensions which are individual characteristics, government factors, risk factors and social media characteristics. These factors are discussed and a summary is presented in this section:

\subsection{Individual characteristics}

The citizen's aspects (trustor) and their beliefs regarding other party influence trust [12, 33]. Many researchers have highlighted propensity or disposition of trust as an important contributors towards trust in e-government [11-13, 34]. Colquitt, Scott and LePine [35] stated that disposition to trust is a personality-based trust which plays a key role in shaping trust even in the presence of trustworthiness information. Disposition of trust refers the general tendency to trust others. It represents the willingness of individuals to engage in a trusting relationship with others based on their own beliefs. This factor differs among individuals based on their personality, experiences and cultural values [36]. The importance of disposition to trust was also validated by Colesca [24] in context of citizens trust in e-government services. According to Bélanger and Carter [13], some people tend to trust on other things or online entities even without having much information about them, whereas some require great deal on information to place their trust on any target. So, high level of disposition of trust increases trust and low level of disposition to trust 
decreases trust. This is especially important in initial phases of building relationships [13]. Using social media services in government organizations is still in its infancy, thus disposition of trust can have some impact on citizen's trust on these services. Hence this paper also considers disposition to trust as a significant factor in context of using government social media services.

\subsection{Government factors}

Citizen-Government relationship plays a central role in forming trust towards adoption of government services [37]. Many researchers agree that trust in government and its institutions is a very significant dimension leading to the successful adoption of government services $[12,19,38]$. Previous literature has interpreted trust in three sub dimensions: Ability, integrity and benevolence. Ability refers to the skills and competencies of government organizations to influence different stakeholders within some domain. Benevolence represents the extent to which a trustee (government organizations) was to do good for the trustor (citizens) apart from any motive of benefits or profits. Integrity represents the extent to which citizens believe that government organizations will adhere to a set of rules or principles which may include their truthfulness, promise fulfilment and justice [35]. Gefen, Karahanna and Straub [39] stated that these beliefs can generate trust in individuals in which they do not feel vulnerable toward risk in online environment with government organizations. Similarly, Scherer and Wimmer [34] and Bélanger and Carter [13] also mentioned Ability, Benevolence and Integrity as significant factors of perceived trustworthiness. Thus, based on the above discussion these government factors should directly affect citizen's trust in using government social media services.

\subsection{Risk factors}

Risk may be described as a fear of losing personal information and fear of being monitored on the Internet [38]. A strong correlation between risk and trust has been identified in previous studies of trust relationship on online government and social networking services [12]. Two important technological risks have been found more important in context of online services which are security and privacy [12]. Privacy risk relates to the loss of control over personal information whereas, security is related with unauthorized access to individual's personal data is using government social media services. Ayyash, Ahmad and Singh [40] indicated the positive influence of both security and privacy in context of e-government services. Similarly, Ranaweera [25] also found a positive relationship between perceived security and perceived privacy on citizens trust in e-government services. This means that the more users perceived their security and privacy in using government social media services, the more they tend to trust in using these services. Using social media for government services is a new phenomenon and citizens might feel concerns in using this technology to use government services. Therefore, this paper also considers these technological risks, which are security and privacy as an antecedent of trust in using government social media services.

\subsection{Social media characteristics}

Social media is used as a technology platform in context of government social media services. According to Beldad, et al. [33] characteristics of any technology influence citizens trust in using that technology. Similarly, Park, et al. [8] mentioned that citizen's trust towards the government can increase if the government understands the characteristics of social media and then utilize them appropriately. Therefore, it's 
important to identify those technological characteristics which may influence citizens trust in using government social media services.

Structural assurance leads to Institution based trust [28], which ensures that there is existing support (legal, contractual or physical) to use a technology. This relates positively in generating trust towards any specific technology. According to McKnight, et al. [29] structural assurance reflects that account and privacy settings and policies are designed and in place in the technology to keep user's information safe. Structural assurance promotes confidence because users believe that there are policies and mechanism in place to keep their critical information safe from unauthorized access. This helps in addressing user's concerns regarding protection form uncertainties in using a technology [29]. The importance of structural assurance and its relationship with trust has been validated in different domain of online services [29, 39]. In context of government social media, government organizations post a lot of information about their services and other activities so there might be a need of structural assurance to attract a good number of citizens to participate.

Previous research has indicated that information quality has a significant impact on citizens trust. For instance, Lee and Lei [41] stated that citizen's perception about usefulness of e-government service is related in terms to access their required information more effectively than traditional ways. Similarly, authors like Nicolaou and McKnight [42], Park, et al. [8] and Weerakkody, Irani, Lee, Hindi and Osman [43] stated good quality information as a significant antecedent to generate trust. The results of previous studies have found that if the e-government website provides accurate and timely information, users tend to use that technology for government services. According to Ayyash, et al. [40], a good information quality information (timely, up to date, comprehensive, relevant) will develop citizens trust to use e-government services. There are variety of information on social networking services generated by multiple users. Thus, it can be argued that reliable, relevant and timely information can affect citizen's trust and can be considered as antecedents of trust in government social media services. Perceived ease of use and perceived usefulness are two main constructs in the Technology acceptance model (TAM). In this study perceived ease of use is described as the degree to which citizen's feel that using government social media services is easy to use and free from efforts. Perceived usefulness in this study represents the degree to which citizen's believe that using government social media services is better and more effective than traditional ways or using static e-government websites for getting government information and services [44]. Davis [22] stated that both of these perceptions shape the intention to use, which in turn shapes the actual use of the technology. Previous studies have found a positive relationship of perceived ease of use and perceived usefulness towards citizens trust [26, 40]. For instance, Hassanein and Head [44] identified perceived ease of use and perceived usefulness as important determinants of online trust. Similarly, Alsaghier and Hussain [23] also validated a positive relationship of perceived ease of use and perceived usefulness with trust in egovernment websites. This paper also considers both perceived ease of use and perceived usefulness as antecedents of citizens trust in context of using government social media services. In addition, based on previous studies using TAM, this paper also considers a direct effect of both components (perceived ease of use and perceived usefulness) with citizens intention to participate in context of government social media services. 
Based on the above discussion, this paper identifies Individual characteristics, government factors, perceived risk and social media characteristics as antecedents of trust in government social media services. The findings illustrated from above discussion are summarized in Table 1.

Table 1. Factors influencing citizen's trust in government social media services.

\begin{tabular}{|l|l|l|}
\hline Antecedents of Trust & Constructs & Sources \\
\hline \multirow{3}{*}{ Individual characteristics } & Disposition to Trust & $\begin{array}{l}{[13,23,24,35,36,} \\
39]\end{array}$ \\
\hline \multirow{4}{*}{$\begin{array}{l}\text { Sovernment factors } \\
\text { characteristics factors }\end{array}$} & Ability & {$[29,35,45,46]$} \\
\cline { 2 - 3 } & Benevolence & {$[29,35,45,46]$} \\
\cline { 2 - 3 } & Integrity & {$[29,35,45,46]$} \\
\hline \multirow{3}{*}{ media } & Security risk & {$[14,25,40,47]$} \\
\cline { 2 - 3 } & Privacy risk & {$[14,25,40,47]$} \\
\cline { 2 - 3 } & Information quality & {$[29,25,40,43]$} \\
\cline { 2 - 3 } & Structural assurances & {$[23,26,39,40,44]$} \\
\cline { 2 - 3 } & Perceived Ease of Use & {$[23,40,44]$} \\
\cline { 2 - 3 } & Perceived Usefulness & \\
\hline
\end{tabular}

\section{PROPOSED RESEARCH MODEL}

An integrated model has been developed based on the relevant literature about trust factors in e-government adoption, social networking sites and related studies about government social media services. The model illustrated in Figure 1 provides a holistic view of citizen's trust factors in government social media services from multiple dimensions. These are Individual characteristics, Government factors, Risk factors and Social media characteristics, identified as antecedents of trust in government social media. Both TAM constructs i.e. Perceived ease of use and Perceived usefulness directly influence trust in government social media services and are directly related towards Intention to participate in these services. Furthermore, this study also contributes to examine the influence of trust on citizen's intention to participate in government social media services. The literature has illustrated a positive connection between trust and citizens intention to adopt a technology. The findings from the study of Alsaghier and Hussain [23] show a significant positive effect of trust in e- government service on citizen's intention to use these services. Similarly, the study conducted by Abu-Shanab [14] also found a significant relationship of trust on citizen's intention to adopt e-government services. Moreover, Scherer and Wimmer [34] claimed that social trust in e-participation positively affects citizen's usage intention. They argued that citizens are more likely to engage in e-participation activities if they trust their governments to take their opinions into consideration. The lack of trust will discourage their level of e-participation. Furthermore, Alharbi, et al. [30] also found that trust in government, internet and social trust factors had a significant effect on Saudi citizens' intention to engage in eparticipation on e-government websites. Based on the above discussion this can be argued that developing trust is an essential requirement to shape citizens behaviour is using e-government services. Therefore, this paper presents trust as a significant predictor towards citizen's intentions to participate in government social media services. 


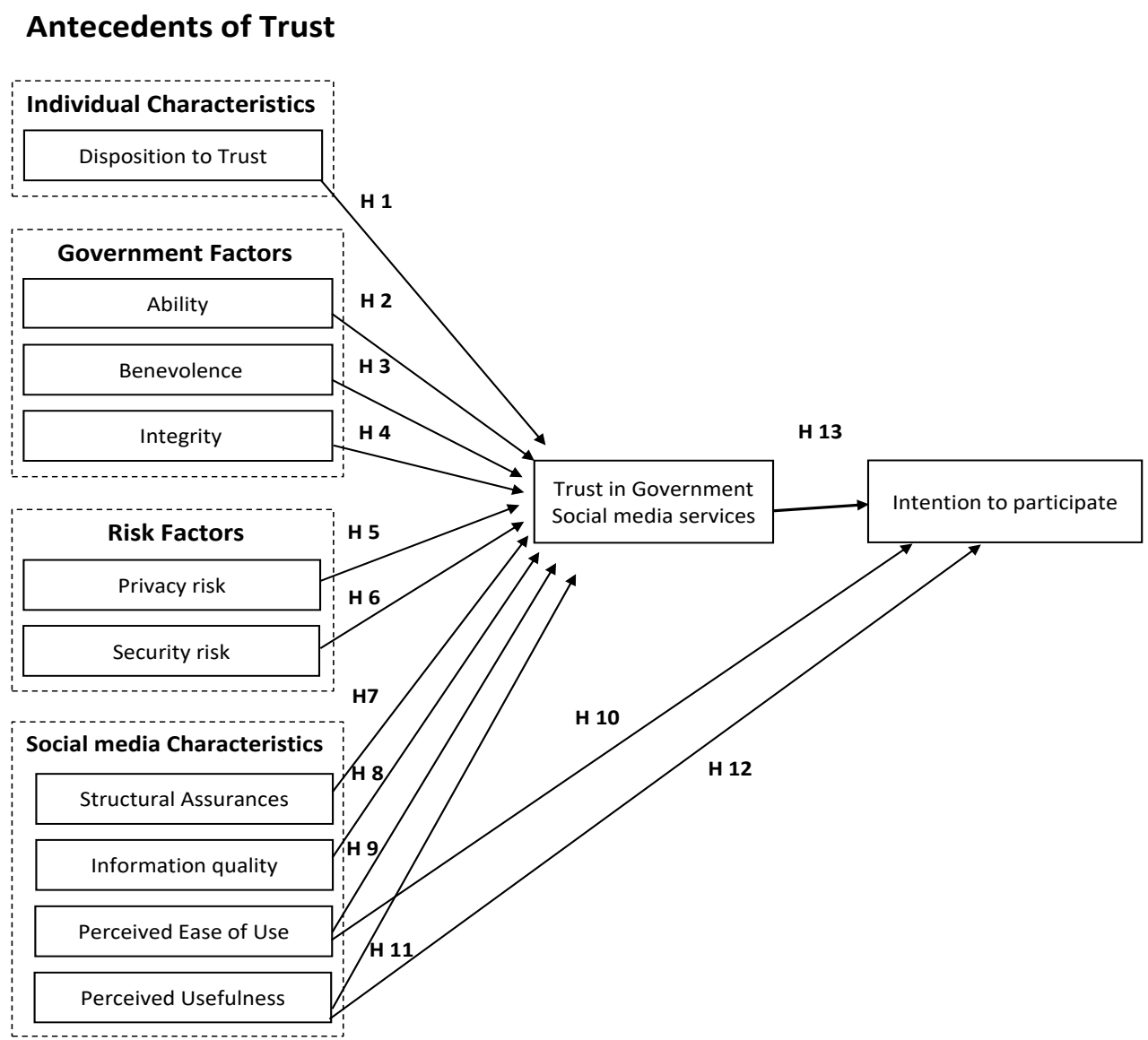

Figure 1. Antecedents of Trust in government social media services.

\section{CONCLUSIONS, CONTRIBUTIONS AND LIMITATION}

Government social media has been accepted as a practice of e-government services across the world. While majority of prior research was conducted on e-government websites with limited focus only on technology and government factors, this paper contributes to explore the factors influencing citizen's trust in government social media from multiple dimensions. This paper identifies four factors that are citizen's characteristics, government factors, risk factors and social media characteristics, as antecedents of citizen's trust in government social media. This paper further presents the impact of citizens trust towards their intention to participate in government social media services. The antecedents of citizen's trust in government social media services must be investigated to enable government organizations to enhance their abilities to devise policies and strategies based on citizen's needs and their willingness to participate in government social media services. This paper aims to contribute at both a theoretical and empirical level towards an improved understanding of using social media in the government context. The antecedents in the proposed model are derived from previous empirical evidences and theories. This makes the proposed model more reliable and viable that creates a basis upon which studies may be conducted to identify citizen's trust factors with different samples of different nations for making assessment and comparisons from the collected data.

There are some limitations in this paper. The first limitation of this paper is its restriction to focus only on citizen's trust perspective towards government social media services. This paper does not cover government organizations, policy maker's perspective regarding 
their social media plans, strategies or any other associated challenges in social media implementation and maintenance issues. In addition, the relationships between various trust antecedents with each other is also not covered in this study.

\section{REFERENCES}

[1] P. B. Shah and N. Lim, Using Social Media to Increase E-Government Adoption in Developing Countries, Proceedings of the 5th International Conference on Theory and Practice of Electronic Governance, ICEGOV '11, (2011), Tallin, Estonia.

[2] G. F. Khan, The Government 2.0 utilization model and implementation scenarios, Information Development, vol. 31, no. 2, pp. 135-149, (2015).

[3] J. W. F. Leung, Social media in e Government, An empirical study of adoption factors and consequences, The Univeristy of Newcastle Australia, (2014).

[4] X. Hao, D. Zheng, and Q. Zeng, How to strenghthen social media interactivity of egovernment, Online Information Review, Emerald Group Publishing Limited, vol. 40, no. 1, pp. 79-96, (2016).

[5] H. Hong, Government websites and social media's influence on government-public relationships, Public Relations Review, vol. 39, no. 4, pp. 346-356, (2013).

[6] M. J. Park, D. Kang, J. J. Rho, and D. H. Lee, Policy role of social media in developing public trust: Twitter communication with government leaders, Public Management Review, vol. 18, no. 9, pp. 1265-1288, (2016).

[7] UN, United Nations E Government Survey: E-Government for the Future we want. Department of Economic and Social Affairs, New York, 2014.

[8] J. M. Park, H. Choi, K. S. Kim, and J. J. Rho, Trust in government's social media service and citizen's patronage behavior, Telematics and Informatics, vol. 32, pp. 629-641, (2015).

[9] A. M. Warren, A. Sulaiman, and N. I. Jaafar, Social media effects on fostering online civic engagement and building citizen trust and trust in institutions, Government Information Quarterly, vol. 31, no. 2, pp. 291-301, (2014).

[10] C. Song and J. Lee, Citizens' Use of Social Media in Government, Perceived Transparency, and Trust in Government, Public Performance \& Management Review, vol. 39, no. 2, pp. 430-453, (2016).

[11] G. A. Porumbescu, Comparing the Effects of E-Government and Social Media Use on Trust in Government: Evidence from Seoul, South Korea, Public Management Review, vol. 18, no. 9, pp. 1308-1334, (2016).

[12] L. Alzahrani, W. Al-Karaghouli, and V. Weerakkody, Analysing the critical factors influencing trust in e-government adoption from citizens' perspective: A systematic review and a conceptual framework, International Business Review, vol. 26, no. 1, pp. 164-175, (2017).

[13] F. Bélanger and L. Carter, Trust and risk in e-government adoption, The Journal of Strategic Information Systems, vol. 17, no. 2, pp. 165-176, (2008).

[14] E. Abu-Shanab, Antecedents of trust in e-government services: An empirical test in Jordan, Transforming Government: People, Process and Policy, Article vol. 8, no. 4, pp. 480-499, (2014).

[15] J. V. Chen, R. J. M. Jubilado, E. P. S. Capistrano, and D. C. Yen, Factors affecting online tax filing - An application of the IS Success Model and trust theory, Computers in Human Behavior, vol. 43, pp. 251-262, (2015).

[16] A. Alarabiat, Electronic Participation Through Social Media: Citizens' Adoption Factors at Local Government Level, Proceedings of the 9th International Conference on Theory and Practice of Electronic Governance, (2016), pp. 424-427: ACM. 
[17] R. M. ALotaibi, M. Ramachandran, A.-L. Kor, and A. Hosseinian-Far, Factors Affecting Citizens' use of Social Media to Communicate with the Government: a Proposed Model, Electronic Journal of e-Government, vol. 14, no. 1, (2016).

[18] F. Parveen, N. I. Jaafar, and A. Sulaiman, Role of Social Media on Information Accessibility, 19th Pacific Asia conference on Information Systems (PACIS), (2015).

[19] P. Franks and M. Driskill, Building Trust in Government through Social Media: An InterPARES Trust Research Project, European conference on Social media, (2014), University of Brighton, UK.

[20] S. A. Mousavi and E. Pimenidis, Social Media Applications in e-Government: A Risk Assessment Approach, 14th European Conference on eGovernment, (2014), Romania.

[21] A. M. Al-Khouri, E-government in Arab countries: A 6-staged roadmap to develop the public sector, Journal of management and Strategy, vol. 4, no. 1, p. 80, (2013).

[22] F. D. Davis, Perceived usefulness, perceived ease of use, and user acceptance of information technology, MIS quarterly, pp. 319-340, (1989).

[23] H. Alsaghier and R. Hussain, Conceptualization of trust in the e-government context: A qualitative analysis, in Active Citizen Participation in E-Government: A Global Perspective IGI Global, (2012).

[24] S. E. Colesca, Understanding trust in e-government, Engineering Economics, vol. 63, no. 4, (2009).

[25] H. M. B. P. Ranaweera, Perspective of trust towards e-government initiatives in Sri Lanka, SpringerPlus, journal article vol. 5, no. 1, p. 22, (2016).

[26] D. Belanche, L. V. Casaló, and C. Flavián, Integrating trust and personal values into the Technology Acceptance Model: The case of e-government services adoption, Cuadernos de Economía y Dirección de la Empresa, vol. 15, no. 4, pp. 192-204, (2012).

[27] I.-L. Wu and J.-L. Chen, An extension of Trust and TAM model with TPB in the initial adoption of on-line tax: An empirical study, International Journal of HumanComputer Studies, vol. 62, no. 6, pp. 784-808, (2005).

[28] D. H. Mcknight, M. Carter, J. B. Thatcher, and P. F. Clay, Trust in a specific technology: An investigation of its components and measures, ACM Trans. Manage. Inf. Syst., vol. 2, no. 2, pp. 1-25, (2011).

[29] D. H. McKnight, V. Choudhury, and C. Kacmar, Developing and validating trust measures for e-commerce: An integrative typology, Information systems research, vol. 13, no. 3, pp. 334-359, (2002).

[30] A. Alharbi, K. Kang, and I. Hawryszkiewycz, The Influence of Trust and subjective Norms on Citizens Intentions to Engage in E-participation on E-government Websites, Australian Conference on Information Systems (2015), Adelaide.

[31] W. H. S. Alkhilani and H. Sulaiman, Identification of factors influencing G2C services acceptance in developing countries, International Conference on Information Technology and Multimedia (ICIMU), (2014), Putrajaya, Malaysia.

[32] T. Wang and Y. Lu, Determinants of Trust in E-Government, International Conference on Computational Intelligence and Software Engineering (CiSE) (2010).

[33] A. Beldad, T. Geest, M. Jong, and M. Steehouder, A cue or two and I'll trust you: Determinants of trust in government organizations in terms of their processing and usage of citizens personal information disclosed online, Government information Quarterly, (2012).

[34] S. Scherer and M. A. Wimmer, Conceptualising Trust in E-Participation Contexts, presented at the International Conference on Electronic Participation, (2014),

[35] J. A. Colquitt, B. A. Scott, and J. A. LePine, Trust, trustworthiness, and trust propensity: a meta-analytic test of their unique relationships with risk taking and job performance, Journal of applied psychology, vol. 92, no. 4, p. 909, (2007). 
[36] H. Kivijärvi, A. Leppänen, and P. Hallikainen, Technology Trust: From Antecedents to Perceived Performance Effects, $46^{\text {th }}$ International Conference on System Sciences (HICSS), (2013), Hawaii.

[37] T. S. H. Teo, S. C. Srivastava, and L. Jiang, Trust and electronic government success: An empirical study, (in English), Journal of Management Information Systems, Article vol. 25, no. 3, pp. 99-131, (2009).

[38] M. Rehman, V. Esichaikul, and M. Kamal, Factors influencing e-government adoption in Pakistan, Transforming Government: People, Process and Policy, vol. 6, no. 3, pp. 258-282, (2012).

[39] D. Gefen, E. Karahanna, and D. W. Straub, Trust and TAM in online shopping: an integrated model, MIS quarterly, vol. 27, no. 1, pp. 51-90, (2003).

[40] M. M. Ayyash, K. Ahmad, and D. Singh, Investigating the effect of information systems factors on trust in e-government initiative adoption in palestinian public sector, Research Journal of Applied Sciences, Engineering and Technology, Article vol. 5, no. 15, pp. 3865-3875, (2013).

[41] C. B. P. Lee and U. L. E. Lei, Adoption of e-government services in Macao, 1st international conference on Theory and practice of electronic governance, (2007), Macao, China, 1328102.

[42] A. I. Nicolaou and D. H. McKnight, Perceived information quality in data exchanges: Effects on risk, trust, and intention to use, Information systems research, vol. 17, no. 4, pp. 332-351, (2006).

[43] V. Weerakkody, Z. Irani, H. Lee, N. Hindi, and I. Osman, Are U.K. Citizens Satisfied With E-Government Services? Identifying and Testing Antecedents of Satisfaction, Information Systems Management, vol. 33, no. 4, pp. 331-343, 2016/10/01 (2016).

[44] K. Hassanein and M. M. Head, The influence of product type on online trust, 17th Bled Electronic Commerce Conference (2004), Slovenia.

[45] M. A. Fuller, M. A. Serva, and J. Baroudi, Clarifying the integration of trust and TAM in e-commerce environments: implications for systems design and management, IEEE Transactions on Engineering Management, vol. 57, no. 3, pp. 380-393, (2010).

[46] R. C. Mayer and J. H. Davis, The effect of the performance appraisal system on trust for management: A field quasi-experiment, Journal of applied psychology, vol. 84, no. 1, p. 123, (1999).

[47] S. S. Alam, M. Ahmad, A. A. Khatibi, and M. N. Ahsan, Factors affecting trust in publishing personal information in online social network: An empirical study of Malaysia's Klang Valley users, Geografia: Malaysian Journal of Society and Space, vol. 12, no. 2, pp. 132-143, (2016).

[48] R. Pennington, H. D. Wilcox, and V. Grover, The role of system trust in business-toconsumer transactions, Journal of Management Information Systems, vol. 20, no. 3, pp. 197-226, (2003). 


\section{SIMULATIVE INVESTIGATION OF TRANSMISSION PERFORMANCE FOR PROPOSED NG-PON 2 BASED ON DPQSK MODULATED DOWNSTREAM WITH TWO DIFFERENT INTE NSITY MODULATED UPSTREAMS}

Areez Khalil Memon

FEST, Indus University, Karachi, (Pakistan)

E-mail: areez.memon@indus.edu.pk

Ahmed Muddassir Khan

FEST, Indus University, Karachi, (Pakistan)

E-mail: ahmed.muddassir@indus.edu.pk

Syed Hyder Abbas Musavi

FEST, Indus University, Karachi, (Pakistan)

E-mail: Dean@indus.edu.pk

Faizan ur Rehman

FEST, Indus University, Karachi, (Pakistan)

E-mail: faizan.rehman@indus.edu.pk

Atif Mehmood

FEST, Indus University, Karachi, (Pakistan)

E-mail: atifmehmood@indus.edu.pk

Sajid Hussain

FEST, Indus University, Karachi, (Pakistan) E-mail: sajidhussain@indus.edu.pk 


\section{ABSTRACT}

We present a comparison between the two high data rate next generation passive optical network (NG-PON 2) which are feasible to fulfill the future demand for bandwidth hungry applications that exceeds beyond 10Gbps. This paper summarizes the best suitable network for future bandwidth hungry applications by simulating both networks through optisys simulated software considering standard values and recommendations for NG-PON 2. Both networks support 40Gbps with Differential Quadrature Phase Shift Keying (DQPSK) while at upstream one network supports 10Gbps with On Off Keying (OOK) and other support Inverted return to zero (IRZ) with $10 \mathrm{Km}$ fiber span. Comparison of both networks transmission performance in terms of BER with Rx power and OSNR has been investigated and results shows that NG-PON 2 support 40Gbps with DQPSK and 10Gbps IRZ is quite better than other network with higher sensitivity and OSNR.

\section{KEYWORDS}

Differential Quadrature Phase Shift Keying (DQPSK), ON-OFF Keying (OOK), Inverted Return to Zero (IRZ), Next Generation Passive Optical Network-2 (NGPON-2), Bit Error Rate (BER), Optical Signal to Noise Ratio (OSNR).

\section{INTRODUCTION}

Evolution in PON is essential to deal with future bandwidth hungry applications. Existing PON have a capability to transmit 10Gbps and $1 \mathrm{Gpbs}$ downstream and upstream data rate with HDTV, multimedia and video calling simultaneously [1-2]. As per the survey of CISCO in 2016 it is predicted that data rate will be increased twice compare to 2015 data rate [3]. It is necessary to increase the data rate capability up to $40 \mathrm{Gpbs}$ in PON to deal with future bandwidth hungry applications and named as NG-PON 2 [4-6].

For higher data rate in PON, different modulation formats have been investigated and analyzed in order to have less nonlinearities and dispersion effects [7]. Different modulation formats have been implemented in 10Gbps data rate such as NRZ, RZ, MDRZ [8], CSRZ, MDRZ, DPSK [9], RZ-DQPSK with OOK [10], Differential Phase Shift Keying (DPSK) with OOK [11], Carrier suppressed return to zero CSRZ-DQPSK with OOK [10-12], NRZ-DQPSK with ASK [13,14], DPSK with IRZ [15,16]. For high data rate, few modulation techniques have been proposed and analyzed such as DQPSK with IRZ [17] and DQPSK with OOK [18].

In this paper we have analyzed the performance of two high data rate NG-PON 2 having the same downstream modulation DQPSK but different upstream modulation OOK and IRZ. DQPSK modulation format is used as it has high spectrum efficiency and high transmission impairments [19] and OOK and IRZ modulation formats used as they are simple and cost effective design [20,21]. Both DQPSK with OOK and DQPSK with IRZ architectures have $40 \mathrm{Gbps}$ data rate in downstream and $10 \mathrm{Gpbs}$ data rate in upstream with $10 \mathrm{~km}$ fiber span by using FBG dispersion compensation.

\section{DESCRIPTION OF SIMULATION}

For analyzing the performance of both architectures, simulation software has been used named as Optisystem [22]. Figure 1 and Figure 2 shows the simulated model of high data rate DQPSK with OOK and high data rate DQPSK with IRZ NG-PON 2 having same parameters except different modulation technique in upstream. Both networks have 
OLT, ONU and optical fiber length of $10 \mathrm{Km}$ with FBG dispersion compensator. CW LASER at $0 \mathrm{dBm}$ launch power is used as a carrier source which is modulated with electrical data stream generated from pseudo random generator (PRBS) having data rate 40Gbps through two series connected Lithium Niobate Mach-Zehnder modulator (LiNb MZM). Modulated signal is transmitted through optical fiber span of $10 \mathrm{~km}$ with

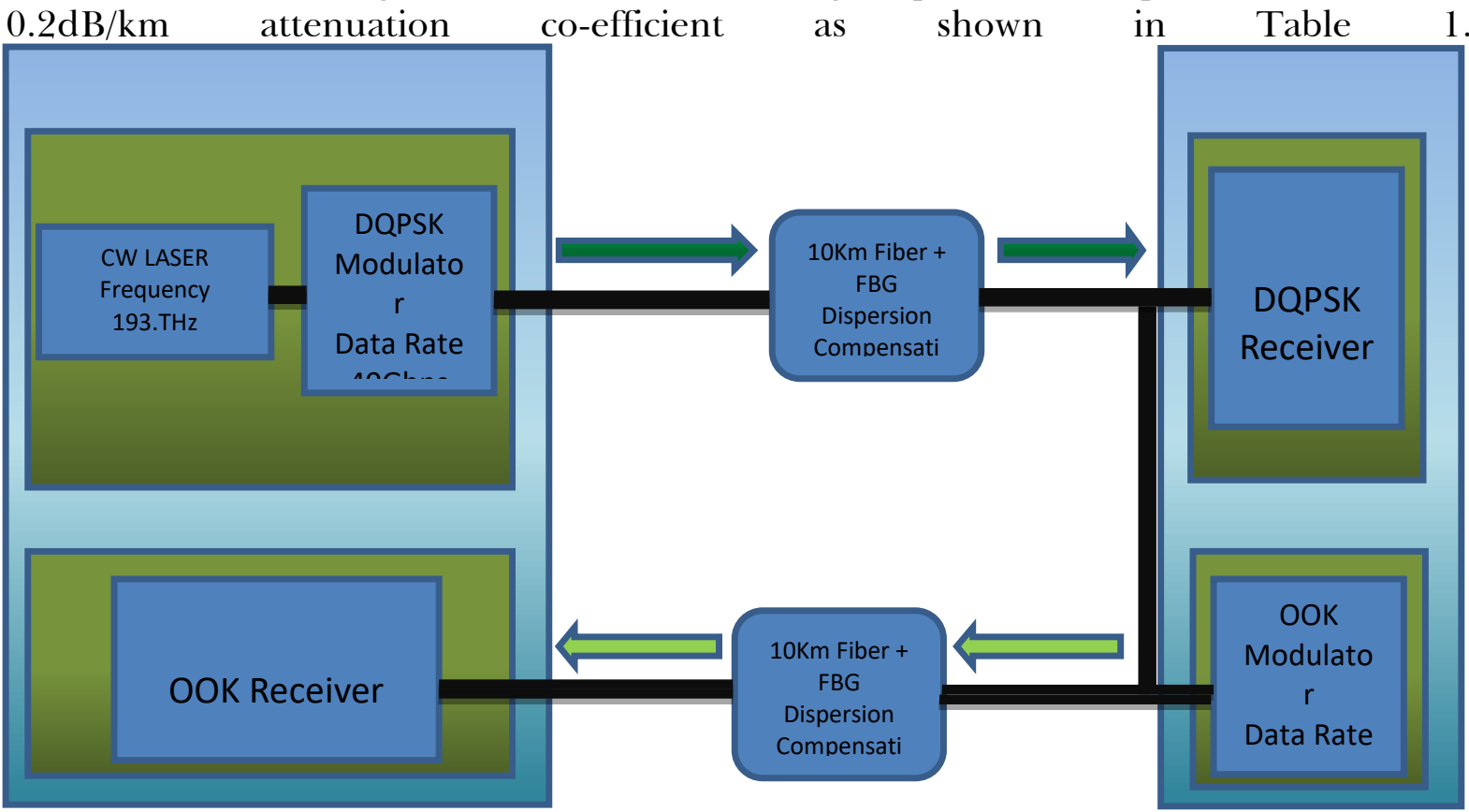

Figure 1. 40Gbps DQPSK with OOK Next Generation Passive Optical Network (NG-PON).

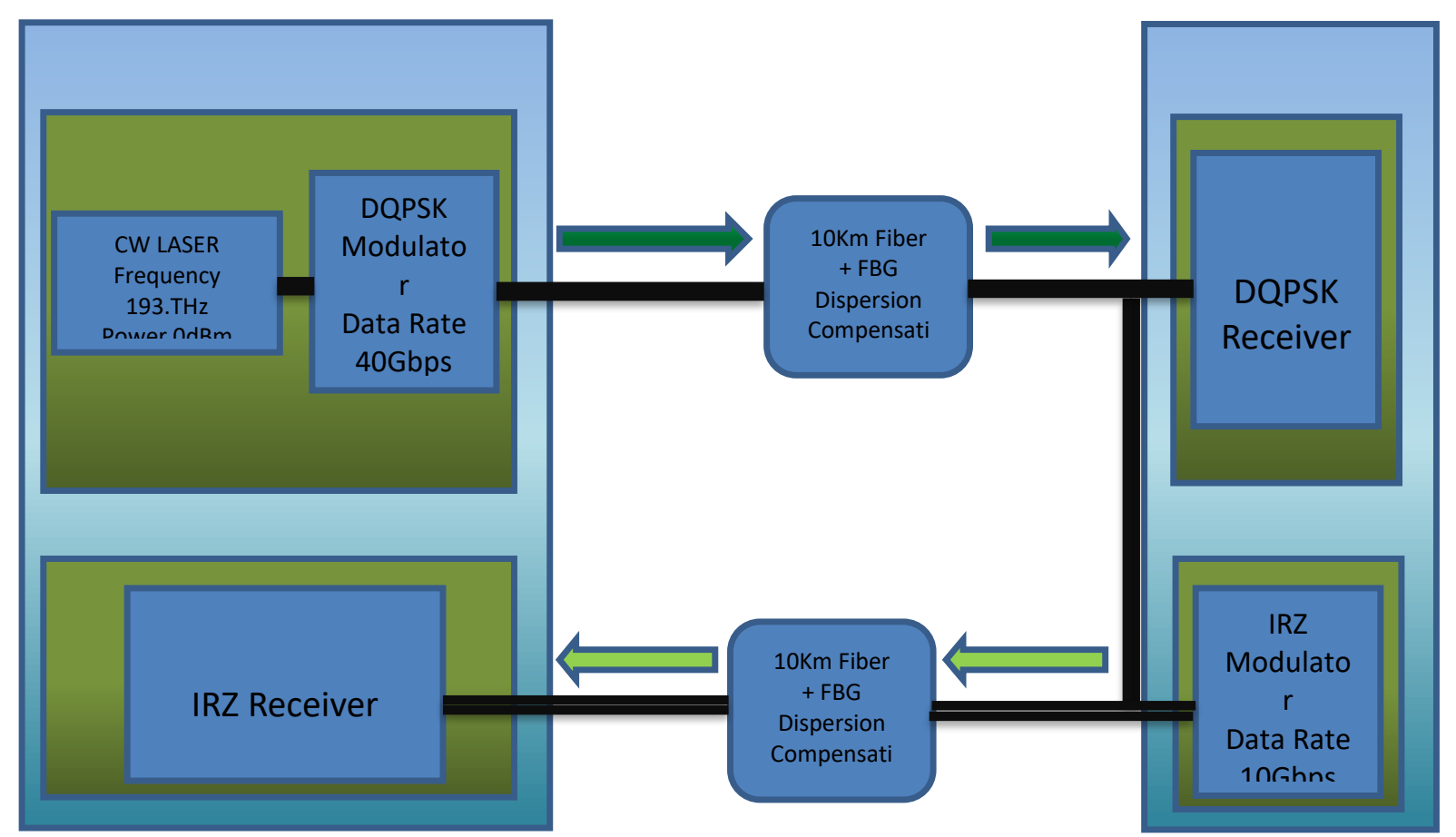

Figure 2. 40Gbps DQPSK with IRZ Next Generation Passive Optical Network (NG-PON) with FBG. 
Table 1. Simulated Model Parameters for both Networks.

\begin{tabular}{|c|c|c|}
\hline \multirow{2}{*}{ Transmission Section } & Parameter & Values \\
\hline \multirow{4}{*}{ Fiber } & Power of Laser & $0 \mathrm{dBm}$ \\
\cline { 2 - 3 } & Frequency of Laser & $193.1 \mathrm{THz}$ \\
\cline { 2 - 3 } & Fiber Length & $10 \mathrm{Km}$ \\
\cline { 2 - 3 } & Dispersion slop & $0.075 \mathrm{ps} / \mathrm{nm}^{2} / \mathrm{km}^{2}$ \\
\cline { 2 - 3 } & Eon Linear index-coefficient & $2.6 \times 10^{-20}$ \\
\cline { 2 - 3 } & Attenuation Coeff: & $0.2 \mathrm{~dB} / \mathrm{km}$ \\
\cline { 2 - 3 } & Dispersion & $16.75 \mathrm{ps} / \mathrm{nm} / \mathrm{km}$ \\
\hline Receiver Section & Filter Cutoff Frequency & $0.75^{*} \mathrm{bit} \mathrm{rate} \mathrm{Hz}$ \\
\hline
\end{tabular}

DQPSK modulation technique is used in the downstream of both networks. DQPSK transmitter modulator is shown in Figure 3. DQPSK transmitter is composed of CW Laser having $0 \mathrm{dBm}$ power $(1 \mathrm{~mW})$ works at $1550 \mathrm{~nm}$ and data is generated from psuedorandom bit squence (PRBS) generator of 40Gbps. Data is modulated after differential precoding through two LiNb Mach-Zehnder Modulators. One modulator is act as a phase modulator to create a dephasing of $\pi / 2$.

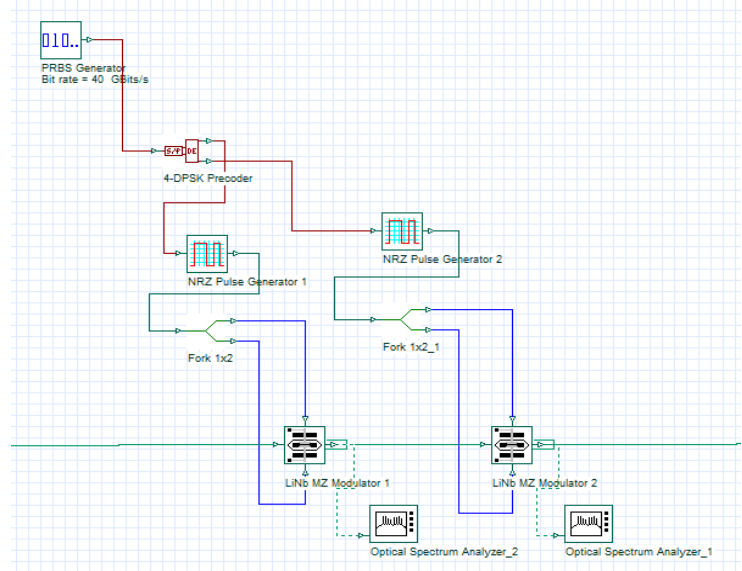

Figure 3. DQPSK Transmitter.

After modulation, signal is transmitted through optical fiber towards the reciever of the ONU where signal is splitted into two signals demodulated through coupler and followed by two photodetector PIN. There output combines with the subtractor and gives the electrical output as shown in Figure 4.

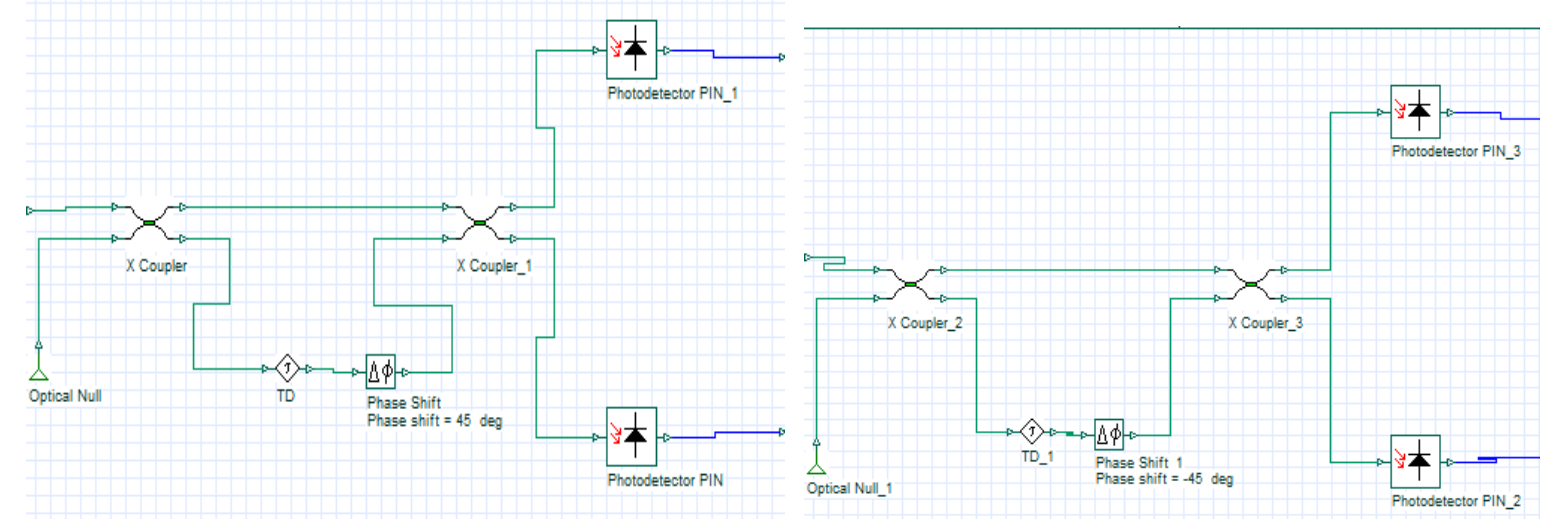

Figure 4. DQPSK Receiver for Inphase and Quadrature phase Signal. 
The ONU transmitter remodulates the signal through OOK modulation in one network while IRZ in another network. For OOK transmitter, same signal is modulated through Mach-Zehnder modulator with data generator from psuedo-random bit squence (PRBS) generator of 10Gbps shown in Figure 5.

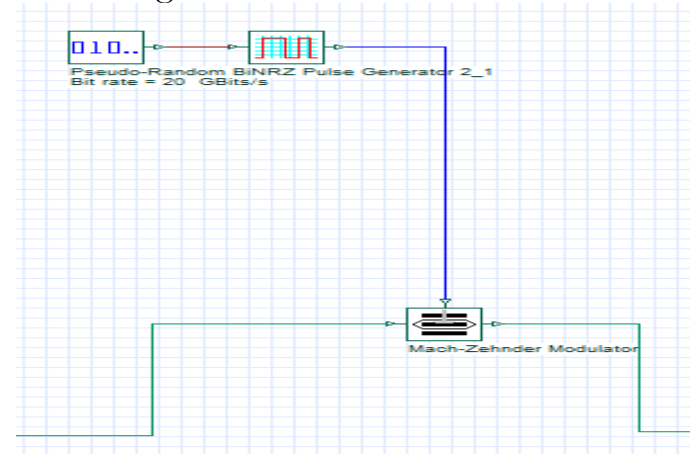

Figure 5. OOK Transmitter.

Modulated signal transmitted through optical fiber received by photodetector which demodulates the signal gives electrical output as shown in Figure 6.

Figure 6. OOK Receiver.

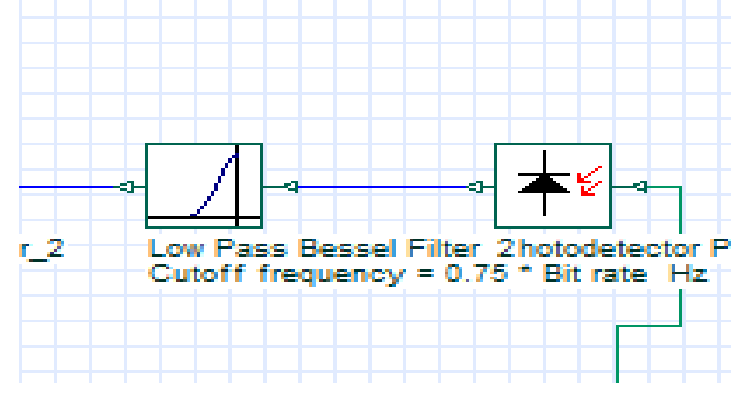

Whereas IRZ transmitter for second network, same signal is modulated through MachZehnder modulator with data generator from psuedo-random bit squence (PRBS) generator of $10 \mathrm{Gbps}$ which added with pulse generator having frequency $5 \mathrm{GHz}$ and phase of $-\pi / 4$ shown in Figure 7.

Figure 7. IRZ Transmitter.

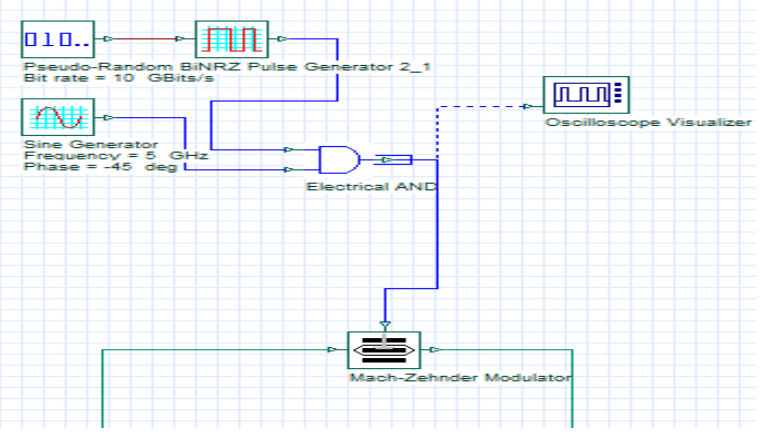

Modulated signal transmitted through optical fiber received by photodetector which demodulates the signal gives electrical output shown in Figure 8.

Figure 8. IRZ Receiver.

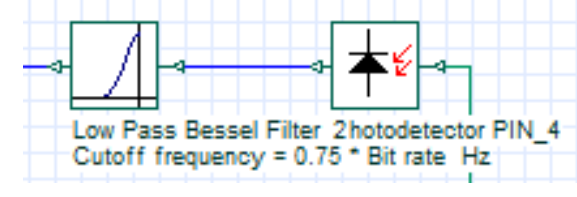




\section{DISCUSSION OF RESULTS}

Figure 9,10,11 and 12 shows the optical spectrum of downstream and upstream of both DQPSK with OOK and DQPSK with IRZ at a carrier frequency of $193.1 \mathrm{THz}(1550 \mathrm{~mm}$ Wavelength).

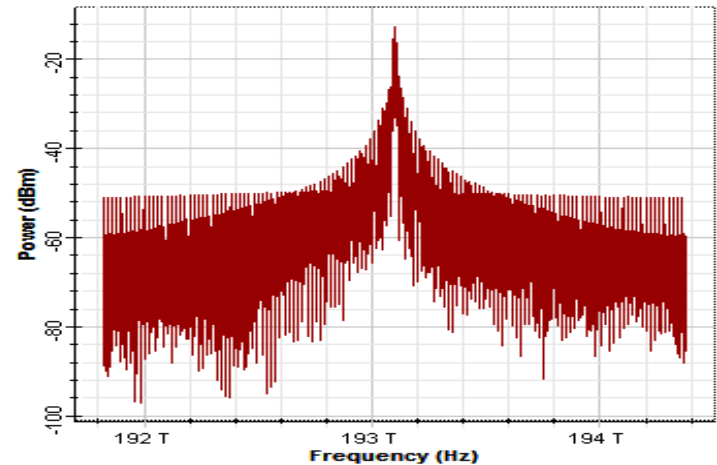

Figure 9. Optical Spectruem of Downstream DQPSK with OOK.

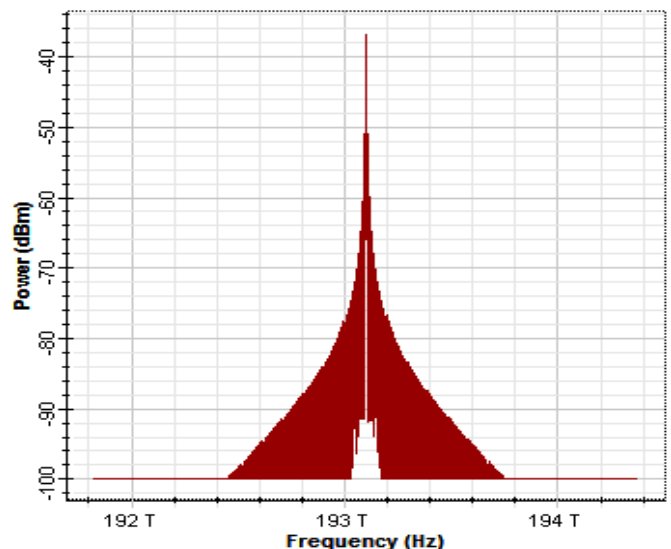

Figure 10. Optical Spectrum of upstream OOK.

\section{를 Optical Spectrum Analyzer_3}

Dbl Click On Objects to open properties. Move Objects with Mouse Drag

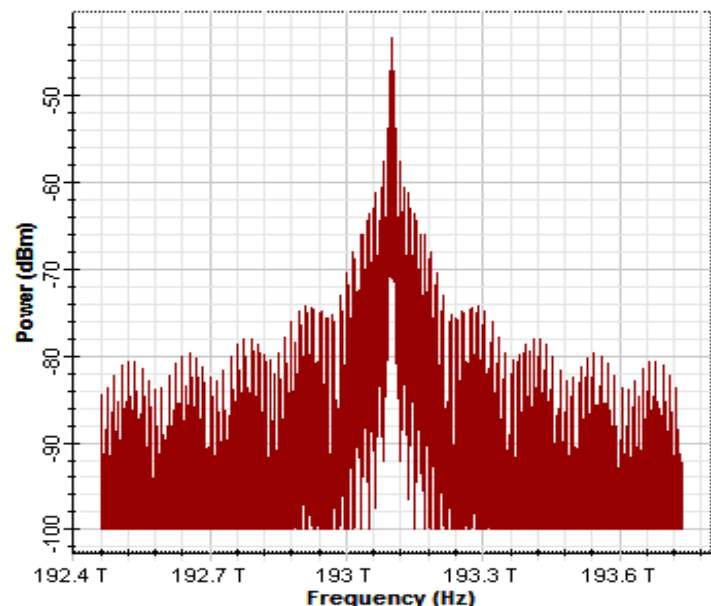

Figure 11. Optical Spectrum of Downstream DQPSK/IRZ. 


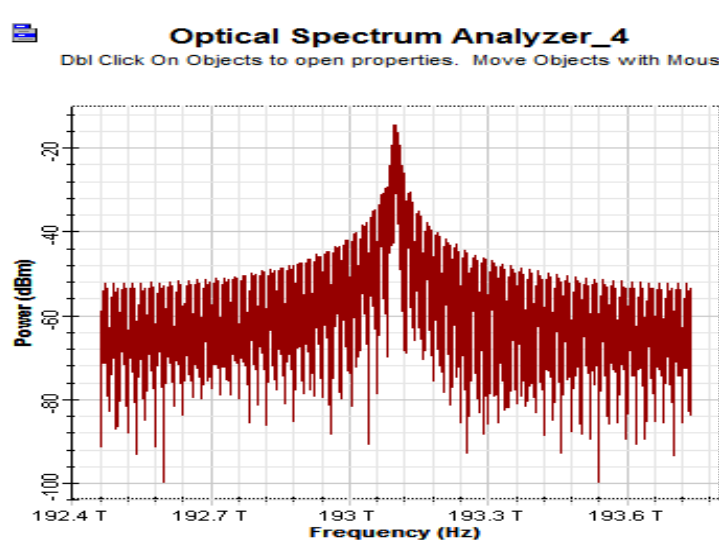

Figure 12. Optical Spectrum of Upstream IRZ.

Figure 13,14,15 and 16 shows the eye diagram of both DQPSK with OOK and DQPSK with IRZ which are wide open having few transmission errors but in an acceptable range to transmit the data. The power and BER of DQPSK/OOK downstream and upstream are $3.41961 \times 10^{-046}$ and $4.10171 \times 10^{-018}$ and $-8.168 \mathrm{~dB}$ and $-34.347 \mathrm{~dB}$ whereas for DQPSK/IRZ are $1.26888 \times 10^{-034}$ and $3.84401 \times 10^{-020}$ and $-8.167 \mathrm{~dB}$ and $-37.824 \mathrm{~dB}$. From the loss margin of both the systems i.e. $-22.832 \mathrm{~dB}$ and $-8.153 \mathrm{~dB}$ for downstream and upstream of DQPSK/OOK and $-34.343 \mathrm{~dB}$ and $-7.676 \mathrm{~dB}$, it can be seen that both systems are feasible for High Data Rate NG-PON 2.

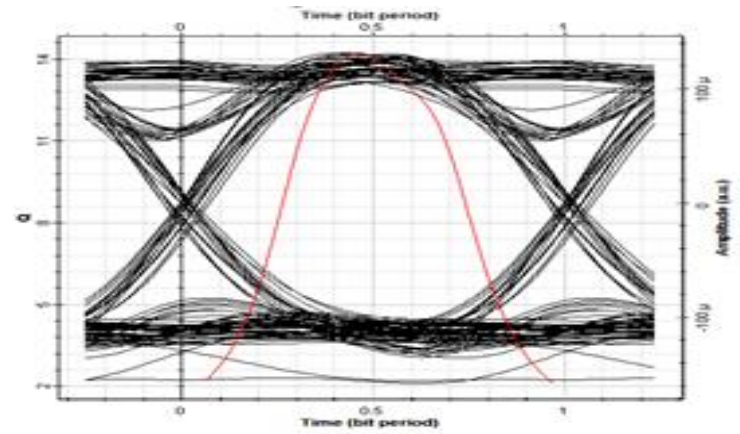

Figure 13. Eye Diagram of Downstream DQPSK with OOK upstream Signal.

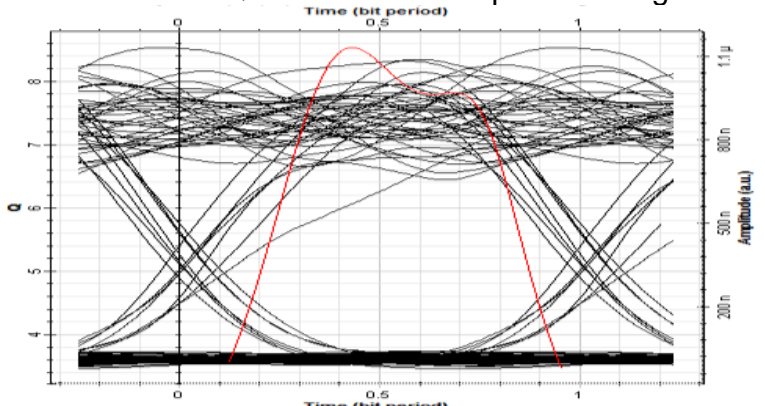

Figure 14. Eye Diagram of Upstream OOK Signal.

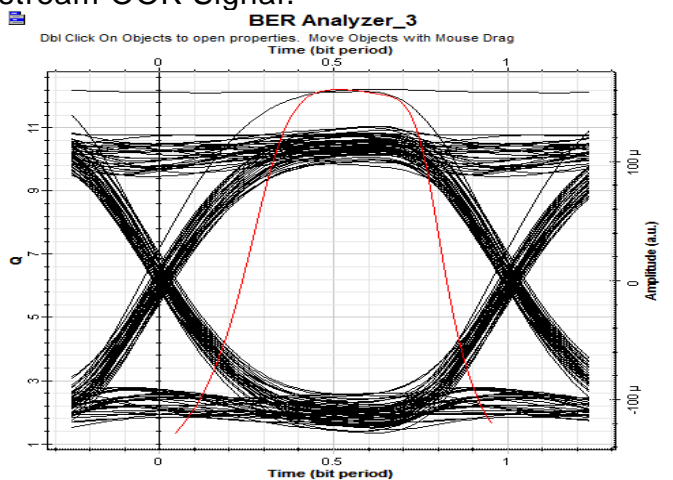

Figure 15. Eye Diagram of Downstream DQPSK with IRZ upstream Signal. 


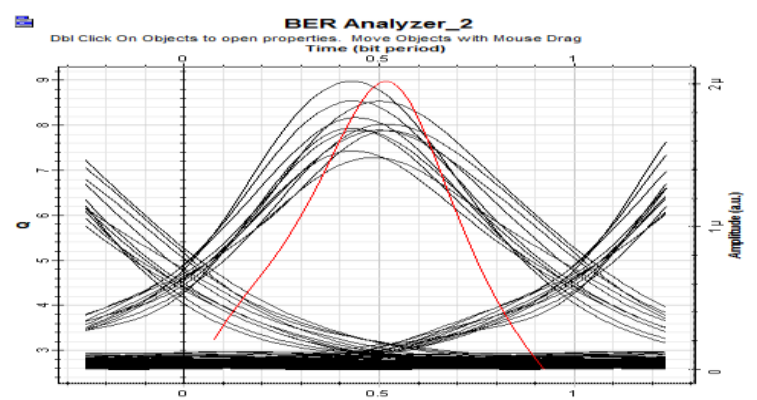

Figure 16. Eye Diagram of Upstream IRZ Signal.

Figure 17 and 18 shows the simulation results obtained on $10 \mathrm{Km}$ and back to back (B2B) fiber span with downstream and upstream of both DQPSK with OOK and DQPSK with IRZ. Figure 17 shows the comparison of downstream of both networks at $10 \mathrm{Km}$ and B2B fiber length. Receiver sensitivity of DQPSK with IRZ at standard BER is much better than DQPSK with OOK on both $10 \mathrm{Km}$ fiber span and B2B i.e. $-31 \mathrm{~dB}$ and $-33 \mathrm{~dB}$ at $10 \mathrm{Km}$ fiber span and $-32 \mathrm{~dB}$ and $-33.5 \mathrm{~dB}$ at B2B. From Figure 17 , it also can be seen that the transmission power penalty of both networks at $10 \mathrm{Km}$ fiber span and $\mathrm{B} 2 \mathrm{~B}$ is $1 \mathrm{~dB}$ and $0.5 \mathrm{~dB}$. Figure 18 shows the comparison of upstream of both networks at $10 \mathrm{Km}$ and $\mathrm{B} 2 \mathrm{~B}$ fiber length. Receiver sensitivity of IRZ at standard BER is much better than OOK on both $10 \mathrm{Km}$ fiber span and B2B i.e. $-38 \mathrm{~dB}$ and $-42.5 \mathrm{~dB}$ at $10 \mathrm{Km}$ fiber span and $-38.5 \mathrm{~dB}$ and $-45.5 \mathrm{~dB}$ at B2B. From Figure 18, it also can be seen that the transmission power penalty of both networks at $10 \mathrm{Km}$ fiber span and $\mathrm{B} 2 \mathrm{~B}$ is $0.5 \mathrm{~dB}$ and $3 \mathrm{~dB}$. Comparison of result is being mentioned in Table 2 .

Table 2. Transmission Performance of DQSPK modulated downstream with OOK and IRZ modulated upstreams.

\begin{tabular}{|c|c|c|c|c|}
\hline NG-PON & \multicolumn{2}{|c|}{ DQPSK with OOK } & \multicolumn{2}{|c|}{ DQPSK with IRZ } \\
\hline & Downstream & Upstream & Downstream & Upstream \\
\hline Fiber Length & $10 \mathrm{Km}$ & $10 \mathrm{Km}$ & $10 \mathrm{Km}$ & $10 \mathrm{Km}$ \\
\hline Rx Power & $-8.168 \mathrm{~dB}$ & $-34.981 \mathrm{~dB}$ & $-8.167 \mathrm{~dB}$ & $-37.82 \mathrm{~dB}$ \\
\hline Bit Error Rate (BER) & $3.42 \mathrm{e}-46$ & $4.10 \mathrm{e}-18$ & $1.27 \mathrm{e}-34$ & $3.84 \mathrm{e}-20$ \\
\hline $\begin{array}{ll}\text { Receiver } & \text { Sensitivity } \\
\text { @ } 1 \times 10^{-9} & \end{array}$ & $-31 d B$ & $-38 d B$ & $-33 d B$ & $-42.5 \mathrm{~dB}$ \\
\hline $\begin{array}{l}\text { Optical Signal to } \\
\text { Noise Ratio (OSNR) }\end{array}$ & $8.52 \mathrm{e} 001$ & $6.47 \mathrm{e} 001$ & $8.68 \mathrm{e} 001$ & $6.03 \mathrm{e} 001$ \\
\hline $\begin{array}{l}\text { Txion Power Penalty } \\
\text { B2B vs } 10 \mathrm{Km}\end{array}$ & $1 \mathrm{~dB}$ & $0.5 \mathrm{~dB}$ & $0.5 \mathrm{~dB}$ & $3 \mathrm{~dB}$ \\
\hline
\end{tabular}


Figure 17. Rx Power vs BER.
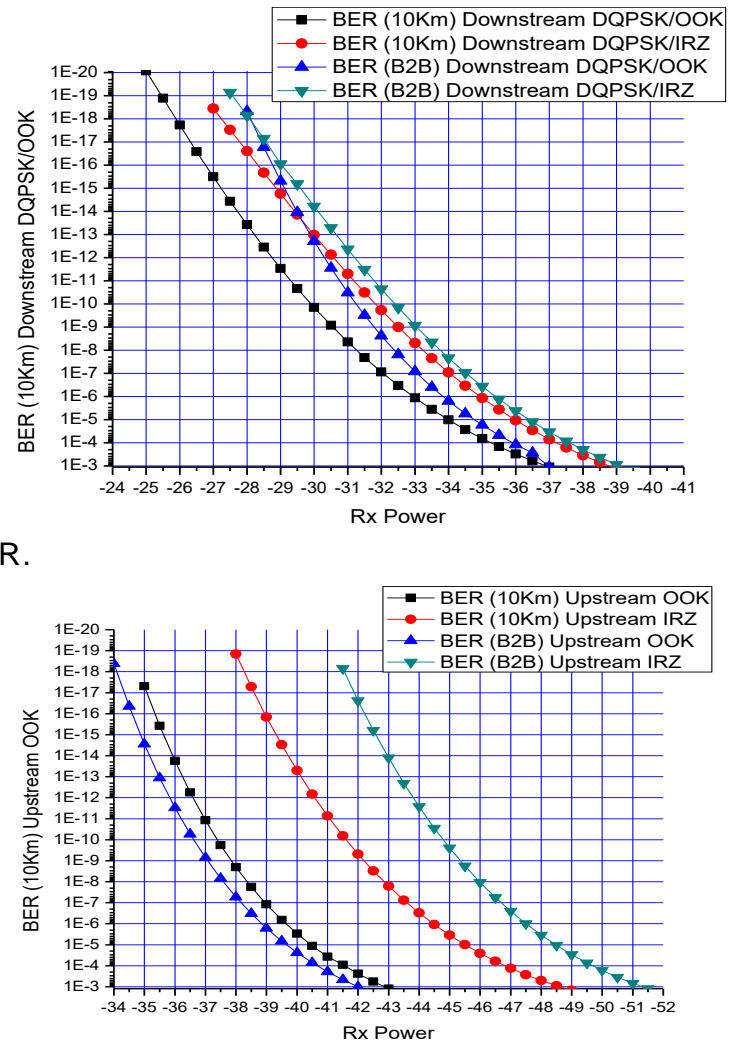

Figure 18. Rx Power vs BER.

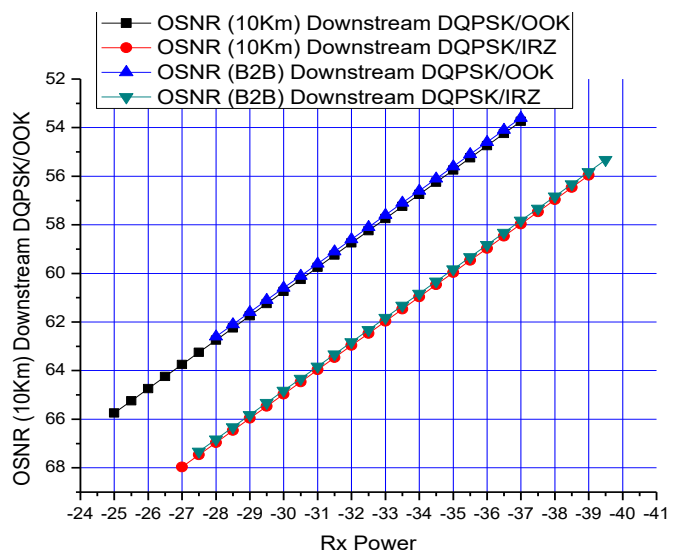

Figure 19. Rx Power vs OSNR.

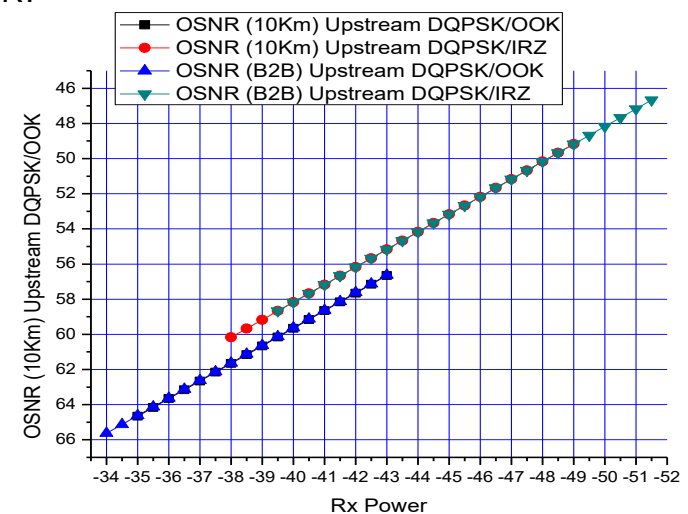

Figure 20. Rx Power vs OSNR.

Figure 19 and 20 shows the simulation results obtained on $10 \mathrm{Km}$ and back to back (B2B) fiber span with downstream and upstream of both DQPSK with OOK and DQPSK with IRZ. Figure 19 shows the comparison of Rx Power vs OSNR at downstream of both 
networks at $10 \mathrm{Km}$ and $\mathrm{B} 2 \mathrm{~B}$ fiber length. It can be seen that as the receiver sensitivity is increased so does OSNR increased. Transmission performance of DQPSK with IRZ at downstream is better compared to DQPSK with OOK as it works at higher receiver sensitivity and OSNR. Figure 20 shows the comparison of Rx power vs OSNR at upstream of both networks at 10Km and B2B fiber length. Here, it can also be seen that IRZ works at higher receiver sensitivity and high OSNR compared to OOK.

From the results, it can be seen that DQPSK with IRZ network is much better than DQPSK with OOK network in terms of performance as receiver sensitivity is high and transmission power penalty is within the standard range.

\section{CONCLUSIONS}

In this paper, we have investigated two high data rate next generation passive optical network 2 (NG-PON 2) with the same modulation DQPSK at downstream but different modulation formats at upstream i.e. one is OOK and another one is IRZ at $10 \mathrm{Km}$ fiber span. Simulated results validate that transmission performance of DQPSK with IRZ is better than DQPSK with OOK in term of higher receiver sensitivity and better OSNR values with very low transmission power penalties in both directions under the similar simulative conditions.

\section{REFERENCES}

[1] Nesset, Derek. (2017), PON Roadmap [Invited]. Journal of Optical Communications and Networking 9.1. A71-A76

[2] Abbas, H. S., \& Gregory, M. A. (2016). The next generation of passive optical networks: A review. Journal of Network and Computer Applications, 67, 53-74.

[3] Cisco Visual Networking Index: Forecast and Methodology, 2016-2021, June 2, 2017. Accessed on 15 June, 2017

[4] Wei, J. L., Grobe, K., \& Griesser, H. (2016, August). High speed next generation passive optical networks: performance, cost, and power dissipation. In Progress in Electromagnetic Research Symposium (PIERS) (pp. 4856-4857). IEEE.

[5] Nesset, D. (2015). NG-PON2 technology and standards. Journal of Lightwave Technology, 33(5), 1136-1143.

[6] Nakamura, H. (2013, March). Ng-pon2 technology. In National Fiber Optic Engineers Conference (pp. NTh4F-5). Optical Society of America.

[7] Tokle, T., Serbay, M., Jensen, J. B., Rosenkranz, W., \& Jeppesen, P. (2008, February). Advanced modulation formats for transmission systems. In Optical Fiber communication/National Fiber Optic Engineers Conference, 2008. OFC/NFOEC 2008. Conference on (pp. 1-3). IEEE.

[8] Rashed, A. N. Z., Mohamed, A. E. N. A., Tabbour, M. S., \& Ismail, A. M. (2017). Performance Improvement for $16 \times 40 \mathrm{~Gb} / \mathrm{s}$ DWDM System Using Non Return to Zero (NRZ), Return-to Zero (RZ) and Modified Duo Binary RZ (MD-RZ) Modulation Formats. International Journal of Advanced Research in Computer Science and Electronics Engineering (IJARCSEE), 6(2), pp-11.

[9] Kamalpreet. Miss Bhawna Utreja May (2015). Performance Analysis of CSRZ, MDRZ, NRZ and DPSK Modulation Formats for Two Channel WDM Passive Optical Network. International Journal of Engineering and Applied Sciences (IJEAS) ISSN: 2394-3661, 
Volume-2, Issue-5 at https://www.ijeas.org/download data/IJEAS0205047.pdf. PDF file.

[10] Garg, A. K., \& Janyani, V. (2015). Analysis of OOK Upstream Signal Remodulation for Different Data Rates in WDM PON Network.

[11] Islam, T. U., Hussain, A., \& Ashraf, S. S. (2015, December). 10Gbps bidirectional transmission GPON network based on single fiber. In Emerging Technologies (ICET), 2015 International Conference on (pp. 1-4). IEEE.

[12] Latal, J., Vitasek, J., Koudelka, P., Siska, P., Poboril, R., Hajek, L., \& Vasinek, V. (2014, July). Simulation of modulation formats for optical access network based on WDMPON. In Transparent Optical Networks (ICTON), 2014 16th International Conference on (pp. 1-7). IEEE.

[13] Chenika, A., Temmar, A., \& Seddiki, O. (2014). Transmission of $4 \times 40 / 10 \mathrm{Gbps}$ in a WDM-PON using NRZ-DQPSK/ASK modulation. Optik-International Journal for Light and Electron Optics, 125(20), 6296-6298.

[14] Chenika, A., Temmar, A., \& Seddiki, O. (2013). A Novel architecture of an optical highspeed access network WDM-PON using NRZ-DQPSK/ASK modulation. ICNCRE'13, ISBN: 978-81-925233-S-5, 1, 396-398.

[15] Khan, A. M., Zhang, J., Zhao, Y., Khan, Y., Latif, A., \& Han, J. (2013). A Cost-effective and Spectrally-efficient Design of Centralized Light Source WDM-PON using Aggregated 160 Gbit/s DQPSK Modulation for Downstream and Re-modulated IRZ for Upstream Transmission. Advances in Information Sciences and Service Sciences, 5(3), 305.

[16] Khan, A. M., Jie, Z., Khan, Y., Idrees, M., Zhao, Y., Niazi, S., \& Liu, J. (2013). A Simple and Cost-effective Design for Simultaneous Transmission of Point-to-point and Broadcast Services in WDM-PON. International Journal of Future Generation Communication and Networking, 6(3), 41-56.

[17] Memon, A. K., Khan, A. M., Musavi, S. H. A., \& Gaho, A. A. (2017). Viable Solution for Next Generation Passive Optical Network 2 (NG-PON 2) Supporting 40Gbps Downstream DQPSK and 10Gbps Upstream OOK. INTERNATIONAL JOURNAL OF FUTURE GENERATION COMMUNICATION AND NETWORKING, 10(8), 29-37.

[18] Das, B., Mukherjee, R., Mandal, G. C., \& Patra, A. S. 40 Gbps Downstream Transmission Using DQPSK and 20 Gbps Upstream Transmission Using IRZ Modulation in FullDuplex WDM-PON. Journal of Optical Communications.

[19] Li, L., Zhang, J., Duan, D., \& Yin, A. (2012). Analysis modulation formats of DQPSK in WDM-PON system. Optik-International Journal for Light and Electron Optics, 123(22), 2050-2055.

[20] YUAN, X. G., ZHANG, J. N., ZHANG, Y. A., ZHANG, M. L., HUANG, Y. Q., \& REN, X. M. (2010). Experimental demonstration and analysis of all-optical label swapping based on RZ-DQPSK/IRZ-ASK modulation format. The Journal of China Universities of Posts and Telecommunications, 17(1), 101-105.

[21] El-Nahal, F. I. (2017). A WDM-PON with DPSK modulated downstream and OOK modulated upstream signals based on symmetric $10 \mathrm{Gbit} / \mathrm{s}$ wavelength reused bidirectional reflective SOA. Optoelectronics Letters, 13(1), 67-69.

[22] https://optiwave.com/ 


\section{AUTHORS}

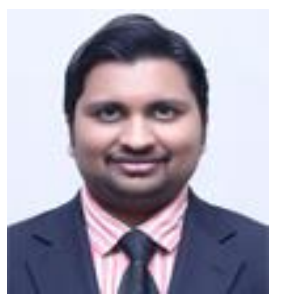

Areez Khalil Memon, He has done B.E(Electronics) from Mehran University of Engineering and Technology, Jamshoro, Sindh, Pakistan (Jan, 2006 - March, 2010) and Masters From University of Leicester, United Kingdom (UK) in MSc Advanced Electrical and Electronics Engineering. Currently, he is doing $\mathrm{PhD}$ in Electrical Engineering from Indus University, Karachi. He has 15 research publications in conferences, IEEE proceedings and journal. A part from his teaching and research activities he is also a member of Board of Studies and Board of Faculty in Indus University. He has good knowledge of Renewable Energy, Electrical Machines, Control Engineering and Passive Optical Networks. His core research areas are Next Generation Passive Optical Network 2 (NG-PON 2) and Energy efficient NG-PON 2.

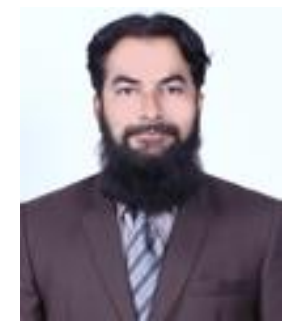

Ahmed Muddassir Khan, he has completed his early education from prestigious institutes of Pakistan with distinctions. He completed B.E. (Electronics) from Mehran University Jamshoro, M.Engg. (Telecommunication) from NED University, Karachi and MBA from Federal Urdu University, Karachi. He started his professional career as a Telecom Engineer in Pakistan's leading Telecom organization (PTCL) and gained vast field and research experience in high capacity optical networks. Then he started PhD from Beijing University of Posts and Telecommunication (BUPT), Beijing, China. His research based on implementation of Robust Advanced Modulation Formats for Cost effective and High capacity Next Generation Passive Optical networks $(\mathrm{PON})$. He has completed $\mathrm{PhD}$ with several research publications in esteemed international research journals. Currently, he is working in Indus University, Karachi, as an Associate Professor and Chairperson of Department of Electrical Engineering in Faculty of Engineering, Science and Technology (FEST). He also has other important academic role and responsibilities in Indus University such as Chair of BOS, member of BOF, Academic Council, and R\&D Committee. He is an approved PhD supervisor and selected as an NTC Program evaluator by higher education commission (HEC), Pakistan. He is also member of different national and internal societies and speaker in different research forums. He served as an organizing committee and technical Co-chair in IEEE ICIEECT 2017. He also has successfully supervised ICT R\&D funded projects His core research areas are Optical Communication, DWDM, GPON, Hybrid WDM/TDM-PON, Submarine Optical Fiber Cable Networks, Advanced Modulation Formats and Next Generation Passive Optical Networks (NGPON). 


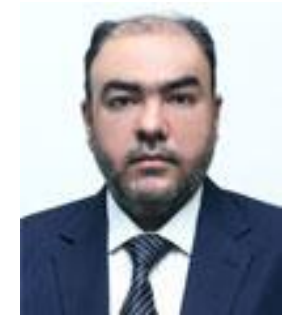

Sayed Hyder Abbas Musavi, He is $\mathrm{PhD}$ and $\mathrm{ME}$ in Telecommunication Engineering under HEC Scholarship and B.E. in Electronics Engineering from Mehran University of Engineering and Technology, Pakistan. He is currently serving as Dean Faculty of Engineering Science and Technology Indus University Karachi. Previously he was engaged as Chairman Department of Electrical and Electronics Engineering Hamdard University Karachi. In past he has served as Professor and Principal at Petroman- an Institute of Ministry of Information Technology and Telecommunications, Government of Pakistan at its various campuses for more than 10 years and had also remained Executive District Officer IT (EDO-IT) District Government Larkana. To his credit are more than 30 research publications in national and international journals. Dr. Musavi has attended numerous international conferences as invited speaker. He is on review board of two impact factor international journals. He is member of numerous national and international societies including member IEEEP Karachi local council, IEEE, IEEE Computer society, IEEE Signal Processing Society, IEEE Devices and Circuits Society, IEEE Communications Society etc. He was General Chair in IEEE ICIEECT 2017.

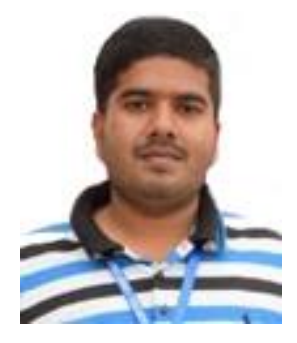

Faizan ur Rehman, Computer Systems Engineer from COMSATS Institute of Information Technology (CIIT) Wah Cantt and Masters in Electrical Engineering from National University of Computer and Emerging Science (NUCES-FAST) Lahore. He is currently working at Indus University Karachi as a Lecturer. His areas of research are Next Generation Passive Optical Networks and Speaker Recognition System.

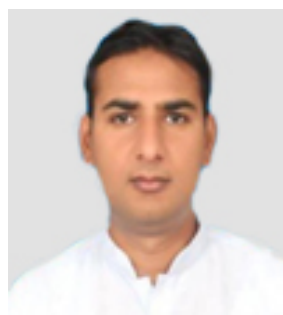

Engr. Atif Mehmood has completed his Bachelor of Computer Engineering from COMSATS IIT Wah cantt and Master in Electrical Engineering from FAST (National University of computer and Emerging Sciences). He joined Commtel System as Field Incharge Engineer and works on HEC Smart University Project in different universities in 2016. He also completed multiple projects in different industries like Bestway Cement, Pakistan Tobacco Company, Askari Cement, Cherat Cement, English Biscuit Manufacturer, Nishat Paper Mill. His areas of research are Next Generation Passive Optical Networks and Parallel Synchronization of Grid Stations.

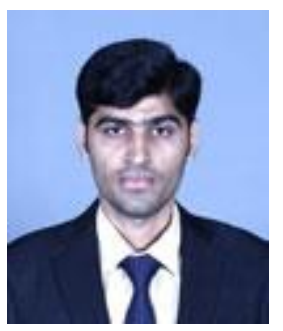

Engr. Sajid Hussain is PEC Certified Engineer with B.E in Electronics. Currently doing M.Engg in Industrial Electronics. Has working experience of around 3 years in different industries. His areas of research are Next Generation Passive Optical Networks. 
Simulative investigation of transmission performance for proposed NG PON 2 based on DPQSK modulated downstream with two diferent intensity modulated upstreams DOI: http://dx.doi.org/10.17993/3ctecno.2019.specialissue.11 


\section{EFFICIENT ENERGY UTILIZATION IN WIRELESS SENSOR NETWORKS: AN ALGORITHM}

M. N. Khan

Department of Electrical Engineering, University of Lahore, Lahore, (Pakistan) *E-mail: muhammad.nasir@ee.uol.edu.pk

S. O. Gilani

SMME, National University of Sciences and Technology, Islamabad, (Pakistan)

M. Jamil

SMME, National University of Sciences and Technology, Islamabad, (Pakistan)

A. Shahzad

Department of Mechanical Engineering, University of Lahore, Lahore, (Pakistan)

A. Raza

Department of Electrical Engineering, University of Lahore, Lahore, (Pakistan) 


\section{ABSTRACT}

In the era of latest technologies, wireless sensor network (WSN) is becoming more popular in many applications: e-health, e-commerce, banking, farming and many others. However, WSNs have limitations in the sense of processing power, life time and data gathering. Among the above said issues, energy efficiency is the main hindrance of WSN deployment. Different data gathering schemes such as low energy adaptive clustering hierarchy (LEACH) and power efficient gathering in sensor information systems (PEGASIS) have been proposed. However, LEACH and PEGASIS do not provide optimal results for the energy consumption problem and are not feasible to implement. In this research paper, energy efficiency in terms of data gathering in WSN is presented. In this research work, a combined flavor of particle swarm optimization (PSO) with simulated annealing (SA) is given. A novel algorithm is proposed in which best chain formation procedure is adopted. Using the proposed algorithm, a balance energy utilization occurred between nodes, which results in increasing the network performance. Simulation results are obtained and compared with other schemes, which shows better performance as compared to LEACH and PEGASIS.

\section{KEYWORDS}

Wireless sensor network (WSN), low energy adaptive clustering hierarchy (LEACH), power efficient gathering in sensor information systems (PEGASIS), base station $(\mathrm{BS})$, sensor nodes $(\mathrm{SN})$.

\section{INTRODUCTION}

In the recent era, wireless sensors have become most prominent subsystem in many applications: e-Health, e-commerce, e-banking, farming, habitat monitoring, forest fire detection [1,2]. Wireless sensor networks (WSNs) are composed of one to many sensor nodes in a sensing area. Sensor nodes (SNs) are deployed in large area comprising of a base station (BS), which is located at variable distances. The SNs locating at larger distance from the BS, consume more energy while communicating or transferring information to the BS. The nodes locating at near distance dissipate less energy while others dissipate more energy. The energy dissipation depends on distance and communication time during data gathering $[3,4]$. Thus energy efficiency in data gathering is a major task to count in WSN design.

In a WSN small SNs are known to be the basic components on which processing is done. SNs are smaller in size, require low power, less memory and least expensive and can communicate over short distance [1,5]. Figure 1 is an example of a WSN, where a large number of SNs (spread in the sensing area) combine to make a fully operational sensor network. The SNs collect data and then forward it to the BS through a leader node (i.e., cluster head $(\mathrm{CH})$ ). The crucial task of SN is to listen an event and respond quickly by sending information to the sink node or BS [5]. 


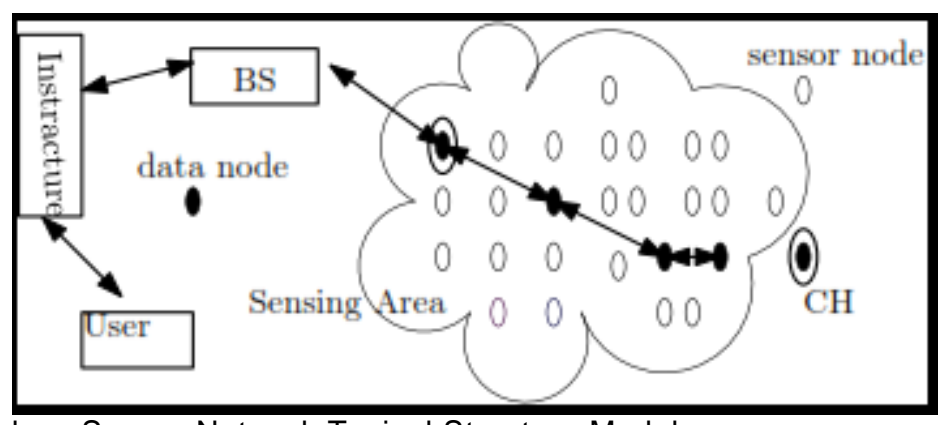

Figure 1. Wireless Sensor Network Typical Structure Model.

SNs in a WSN have very limited energy, it is hard to recharge remotely or change their batteries more often. To cope with this issue, we need to have an energyefficient data gathering protocol for balancing the energy dissipation among SNs of the network. For WSN to be efficient and acceptable, following requirements are very crucial [6-10];

The network lifetime enhancement.

Energy efficient deployment schemes.

Need for an energy-efficient data gathering protocol.

Recently, various data gathering schemes including the low energy adaptive clustering hierarchy (LEACH) [11] and power efficient gathering in sensor information systems (PEGASIS) [12] have been suggested. The LEACH protocol provides solutions for energy utilization problem. In LEACH, SNs with higher energy are chosen as a $\mathrm{CH}$ randomly and $\mathrm{CH}$ communicates to the $\mathrm{BS}$. The $\mathrm{CH}$ forwards data to the $\mathrm{BS}$ also causes energy dissipation which is not required. The un-necessary dissipation limits the applicability of LEACH for WSNs. On the other hand, PEGASIS protocol is proposed for further improvement in LEACH protocol. However, in PEGASIS, greedy chain formation allows all nodes to become the leader at the same time, which needs more rounds in forwarding data to the BS. This way of energy utilization is a big hurdle in PEGASIS implementation.

Both algorithms do not provide optimal results in terms of energy efficiency problem. We present particle swarm optimization (PSO) with simulated annealing (SA) for efficient chain formation and ensures minimum energy consumption. For the selection of efficient leader that will communicate to the sink, data gathering chain for done with SA-PSO. In a chain a leader is selected based on its residual energy $E_{\text {resid }}$ and each node can transmit information to its neighboring node, this result in increased network performance as balance energy utilization occurred between nodes. Following are the major contribution of the proposed research work;

A new scheme for efficient energy is developed and tested as compared to other protocols using extensive simulations. It is shown from simulation results that the proposed protocol can prolong the network lifetime.

The SA-PSO maximizes the performance in terms of energy efficiency and also increases the network lifetime.

In our scheme all the nodes contribute in communication to the BS and thus it eliminates unequal energy consumption of individual nodes.

The simulation results also show significant improvements as compared to LEACH and PEGASIS. Our further goal is to compare the SA-PSO performances with ant colony optimization (ACO) technique. 


\section{PRE-LIMINARIES}

Different data gathering schemes and heuristic algorithms have been suggested in [1-4]. Following are the main ones;

Conventional PSO and its variants.

LEACH and its variants

PEGASIS

Before discussing about the LEACH and PEGASIS, brief description of PSO and its variant are considered to be compulsory, because the proposed algorithm is the combination of PSO and SA.

Basically LEACH is cluster based routing protocol, consists of distributed clusters. In LEACH protocol, $\mathrm{CH}$ is randomly selected and this is constantly rotated to distribute energy load along all node in the entire network. In LEACH protocol, to decrease the load of transmitted data, the $\mathrm{CH}$ node compress data arriving from the sensor nodes and send an aggregated packet of data to the BS [7]. The drawback of LEACH protocol is that it randomizes he selection of cluster heads for equal energy dissipation. Whereas the PEGASIS protocol uses a greedy chain to sink. To overcome these drawbacks, we use particle swarm optimization with simulated annealing (SA).

\subsection{Conventional PSO}

PSO is a swarm intelligence based optimization technique. PSO was first developed in [13], inspiring from the social behavior of flocking birds. A wide variety of particles are exploited to constitute a swarm and it moves in various direction of sensing area for locating the best possible solution. Each SN in the sensing area keeps tracking the coordinates of optimum solution or fitness and is known as the pbest. There is also another value called the gbest that is obtained the particle in the neighborhood. The position $Y$ of each particle $m$ in the given space $D$ is formulate as $[13,14]$,

$Y_{i}=\left[y_{m_{1}}, y_{m_{2}}, \ldots, y_{m_{D}}\right]$.

The previous best position stored by each particle as pbest,

$P_{i}=\left[p_{m_{1}}, p_{m_{2}}, \ldots, p_{m_{D}}\right]$,

and the velocity for each dimension is given by,

$V_{i}=\left[v_{m_{1}}, v_{m_{2}}, \ldots, v_{m_{D}}\right]$.

The position and velocity updates are given respectively as,

$y_{m}=\left[m_{d}+v_{m_{d}}\right]$

$v_{m_{d}}=\left[v_{m_{d}}+s_{1} q_{1}\left(\right.\right.$ pbest $\left.-y_{m_{d}}\right)+s_{1} q_{1}\left(\right.$ gbest $\left.\left.-y_{m_{d}}\right)\right]$,

where $d$ is the dimension, $s_{1}$ and $s_{2}$ denote positive constants, , $q_{1}$ and $q_{2}$ are random numbers between [0-1], $y_{m}$ is position of a particle, $v_{m}$ is velocity of a particle. PSO becomes popular because of its superior performance in may application and was used with combination of other techniques as a hybrid algorithm $[15,16]$. On the other hand, SA proposed in [17] is found to be most extensive optimization technique. It is stochastic process based on Metropolis Law [8] that search for the best optimal solution. It discusses about two factors: - the energy factor and temperature factor $\theta$, where $\theta$ is referred to as annealing temperature. The value of $\theta$ is decreased by the cooling schedule. The region of best point is attained as $\theta$ approaches the lower-limit [1]. 


\subsection{Leach and its variants}

LEACH is a hierarchical routing protocol [11,15] and is a self-organizing scheme. In LEACH, SNs are divided into multiple clusters. Each cluster calculates its $\mathrm{CH}$, which collects data from all neighboring nodes in the cluster. Then $\mathrm{CH}$ forwards the aggregated data to the BS. In this process, $\mathrm{CH}$ consumes more energy if it falls near to the BS, which results its quick death [15]. To overcome this problem, LEACH randomizes the selection of $\mathrm{CH}$, in order to balance the energy dissipation. LEACH can be found in many application $[11,15,18]$.

In LEACH, once the cluster is formed, all the nodes decide to be the $\mathrm{CH}$ with probability $p_{r}$ and broadcast its advertisement to all neighbors. A non-cluster head node finds its cluster by selecting the $\mathrm{CH}$, which has the least energy. To balance the load, the $\mathrm{CH}$ data message is delivered in a sequence of node. When a $\mathrm{CH}$ dies, the cluster is not operated. It is assumed that $\mathrm{CH}$ has long transmission range so that network can have long lifetime. But it is not a good assumption in terms of signal propagation. Although LEACH was found to be useful, but it still has problem of short network lifetime because of inefficiently consumed energy $[15,19]$. To overcome issues various variants of LEACH, i.e., LEACH-C, Multi-hop LEACH, Energy-LEACH, MOD-LEACH and many more were suggested in $[11,20,21,22]$.

\subsection{Pegasis and its variants}

PEGASIS is an improved form also referred to as an extension of LEACH protocol. PEGASIS greedy chain formation based algorithm having chain construction and fusion functions [12]. In PEGASIS, each SN has global information of its whole sensing network and full knowledge of location of its neighboring nodes. The chain formation is initialized by a node over the furthest distance from BS. After the connection of node in chain, all its correspondence turned off from remaining chain formation process.

It is assumed that $\mathrm{SNs}$ are capable to vary the signal strength after getting the global information. By doing so, energy consumption can be minimized up to an extent that the network lifetime is maximized. It is presented that PEGASIS is comparatively more energy efficient as compared to LEACH in the sense of network lifetime [20]. The energy is saved by forwarding the aggregated data rather than bulk data to BS. The drawback of PEGASIS is that due to greedy chain formation process, whole data transmission to BS takes more time, which causes higher latency. PEGASIS have been modified and proposed by many researcher [22-27].

\section{PROPOSED DATA GATHERING SCHEME}

In the proposed energy efficient scheme, a chain is supposed to be formed allowing the transmission of data by individual nodes. This transmission of data is carried out to the BS for unequal number of times. To achieve our design objective, we use PSO [13] and SA [17] that result in energy efficient network by the individual nodes and enhanced network performance.

\subsection{Selection of leader}

An optimal chain formation using SA is presented and the selection of a leader (i.e., $\mathrm{CH}$ ) is done for communication. In our scheme there is data transmission 
between the closed neighbors and the node become the leader in their turn depending on its residual energy and location. In the proposed scheme a chain is formed such that all nodes have equal rights to become the leader. The network lifetime is maximized because of transmitting unequal number of times by individual node to the BS based on its $E_{\text {resid }}$ and thus it eliminates unequal energy consumption of individual nodes, which results in best performance than LEACH and PEGASIS.

Once a sub-optimal chain formation is done for the first time, a node with maximum value of $\frac{E_{\text {resid }}}{D^{\gamma}}$, here $D$ is the distance of the BS from that node, is to be located (i.e., search a node with maximum energy) before initializing the round of data gathering. In this process, a node, which gives the maximum value of $\frac{E_{\text {resid }}}{D}$ is chosen as the leader. The multi-path fading (i.e., distance power loss) channel mode is assumed in case the leader is concerned to communicate with the distant BS.

\subsection{Data gathering algorithm: SA-PSO}

To overcome drawbacks of LEACH and PEGASIS, we present a scheme in which an efficient leader is chosen for communicating to BS. The SA algorithm is combined with PSO to efficiently solve the chain formation problem. Our aim is to minimize the energy usage by $\mathrm{SNs}$ in forming an optimal chain over which the data gathering is done. To simplify the model, we consider the same radio model as is presented in [7].

To eliminate the local minima trapping of the existing algorithm, the proposed method of application of SA with PSO solve two major issues: increase the diversity of the particle and efficiently solve chain formation problem. Let's consider $\$ \mathrm{n} \$$ total nodes and solution space $U$ which is $U$ $\varepsilon\{1,2,3, \ldots, \mathrm{n}\}$ a collection of arrangement and the consecutive nodes are linked together by a direct link. The combination, i.e., $C$ denotes a chain and $C=U_{i} \mid C$ represents a permutation of $(1,2, \ldots \mathrm{n})$.

\section{Proposed algorithm}

\section{Step 1: Initilization.}

Initializing all parameters $\left(\alpha, \theta_{\mathrm{i}}, \theta_{\mathrm{f}}\right)$, where $0.7 \leq \alpha \leq 1$.

The randomly selected swarm of $m$ particles is expressed as $C_{1}, C_{2}, C \ldots C_{m}$

\section{Step 2: Locating a local best chain.}

Search for the local best chain for a given $\theta_{i}$, based on binary swapping resulting $\mathrm{C}_{\text {ilbest }}$ is find out with the help of binary swapping between the two positions of $\mathrm{G}_{\text {old }}$. $\mathrm{C}_{\text {old }}$, updated by the newly formed chain.

$\mathrm{C}_{\text {old }}=\mathrm{C}_{\text {new }}=\mathrm{C}_{\text {ipbest }}=$ Local best solution of particle

\section{Step 3: Updating gbest values.}

The newly formed chain is updated by the following rule:

$$
\begin{gathered}
\mathrm{C}_{\text {ipbest }}=\mathrm{C}_{\text {ilbest }} \text { if }\left\{\mathrm{f}\left(\mathrm{C}_{\text {ilbest }}\right)-\mathrm{f}\left(\mathrm{C}_{\text {ipbest }}\right)\right\}<0 \\
=\mathrm{C}_{\text {ipbest }} \text { if }\left\{\mathrm{f}\left(\mathrm{C}_{\text {ilbest }}\right)-\mathrm{f}\left(C_{\text {ipbest }}\right)\right\} \geq 0 \\
\text { Comparing the } \mathrm{C}_{\text {ipbest }} \text { values } \\
\mathbf{C}_{\text {gbest }}=\boldsymbol{\operatorname { m i n }}\left\{\mathbf{f}\left(\mathbf{C}_{\text {ipbest }}\right)\right\}
\end{gathered}
$$




\section{Step 4: Formation of new chain.}

A new chain $\mathrm{C}_{\text {gbest }}$ is formed from the $\mathrm{C}_{\text {ipbest }}$ by crossing method.

\section{Step 5: Loop.}

The algorithm comes to end if the value of temperature $\theta(t)$ is less than or equal to $\theta_{\mathrm{f}}$. The best chain formed is $C_{\text {gbest }}$,else move to step 2 .

\section{SYSTEM DISSIPATION MODEL}

Radio model also referred to the energy dissipation model is the most common model known in WSNs. Block diagram of the energy dissipation model is shown in Fig. 2. During the processing of different tasks, SNs dissipate energy while transmission and reception of data from/to BS. Let's assume that SNs consume the transmitted energy $E_{T x}$ in transmitting a $k$-bit packet over a distance $d$ meters as shown in Fig. 2.

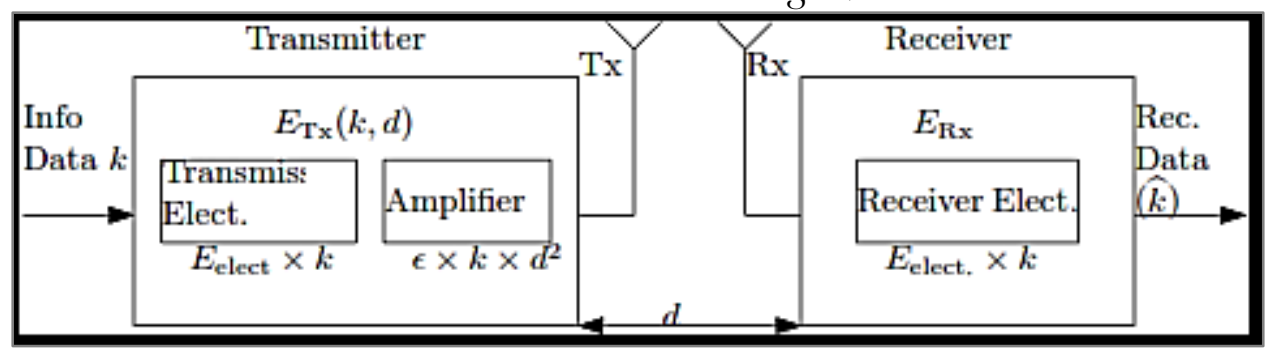

Figure 2. Block diagram of energy dissipation model.

\subsection{Mathematical model}

According to the radio model, $k$-bit message is transmitted covering a distance $d$ and the energy required to transmit the message is given by,

$$
\begin{array}{r}
E_{T x}(k, d)=k E_{\text {elect }}+\epsilon k d^{2} \quad \text { if } d<d_{o}(8) \quad \text { if } d \geq d_{o} \\
=k E_{\text {elect }}+\epsilon k d^{4} \quad \text { if }
\end{array}
$$

where, $E_{\text {elect }}$ represents the dissipated energy by the transmitter or the receiver circuit, $d$ denotes the distance between sender and receiver, $\epsilon$ is the energy dissipated by electronic and $d_{o}$ is the threshold distance. Noted that we consider the effect of free space and multi-path model in the dissipation model. Further to the dissipation model, free space path loss is considered for the transmission/reception between the $\mathrm{CNs}$ and $\mathrm{CH}$, whereas, multi-path model is for the $\mathrm{CH}$ to the BS. Then, the total energy $E_{t}$ consumed by the network for a round is given by $[5,6]$,

$\mathrm{E}_{\text {total }}=\left\{\sum_{\mathrm{i}=1}^{\mathrm{n}-1}\left(\mathrm{E}_{\text {elec }}+\mathrm{E}_{\mathrm{amp}} * \mathrm{~s}_{\mathrm{i}}^{2}\right)+\left(\mathrm{E}_{\text {elec }}+\mathrm{E}_{\mathrm{amp}} * \mathrm{~S}^{4}\right)\right\} * \mathrm{~m}$

where the average distance between the $\mathrm{SNs}$ and $\mathrm{CH}$ is $0.765 * \frac{M}{2}$ and $M$ is the dimension of sensing area [6].

\subsection{Numerical results}

We provide numerical results using the parameters given in Tab.1. To evaluate the performance of the proposed algorithm, large number of simulations were done for a given sensing area and the number of sensing nodes over the given parameters. MATLAB is used as a simulation tools to get results. 
Table 1. Simulation Result Parameters.

\begin{tabular}{|l|l|}
\hline Parameters & Values \\
\hline Sensing area & $100 \times 100$ \\
\hline SNs & 200 \\
\hline Initial E & $0.5 \mathrm{~J}$ \\
\hline Message size & $4 \mathrm{k} \mathrm{bits}$ \\
\hline $\mathrm{E}_{\text {elect }}$ & $50 \mathrm{~nJ} / \mathrm{bit}$ \\
\hline$\epsilon$ & $0.0015 \mathrm{pJ} / \mathrm{bit}$ \\
\hline $\mathrm{d}_{0}$ & $70 \mathrm{~m}$ \\
\hline Data aggregation cost & $5 \mathrm{~nJ} / \mathrm{bit}$ \\
\hline
\end{tabular}

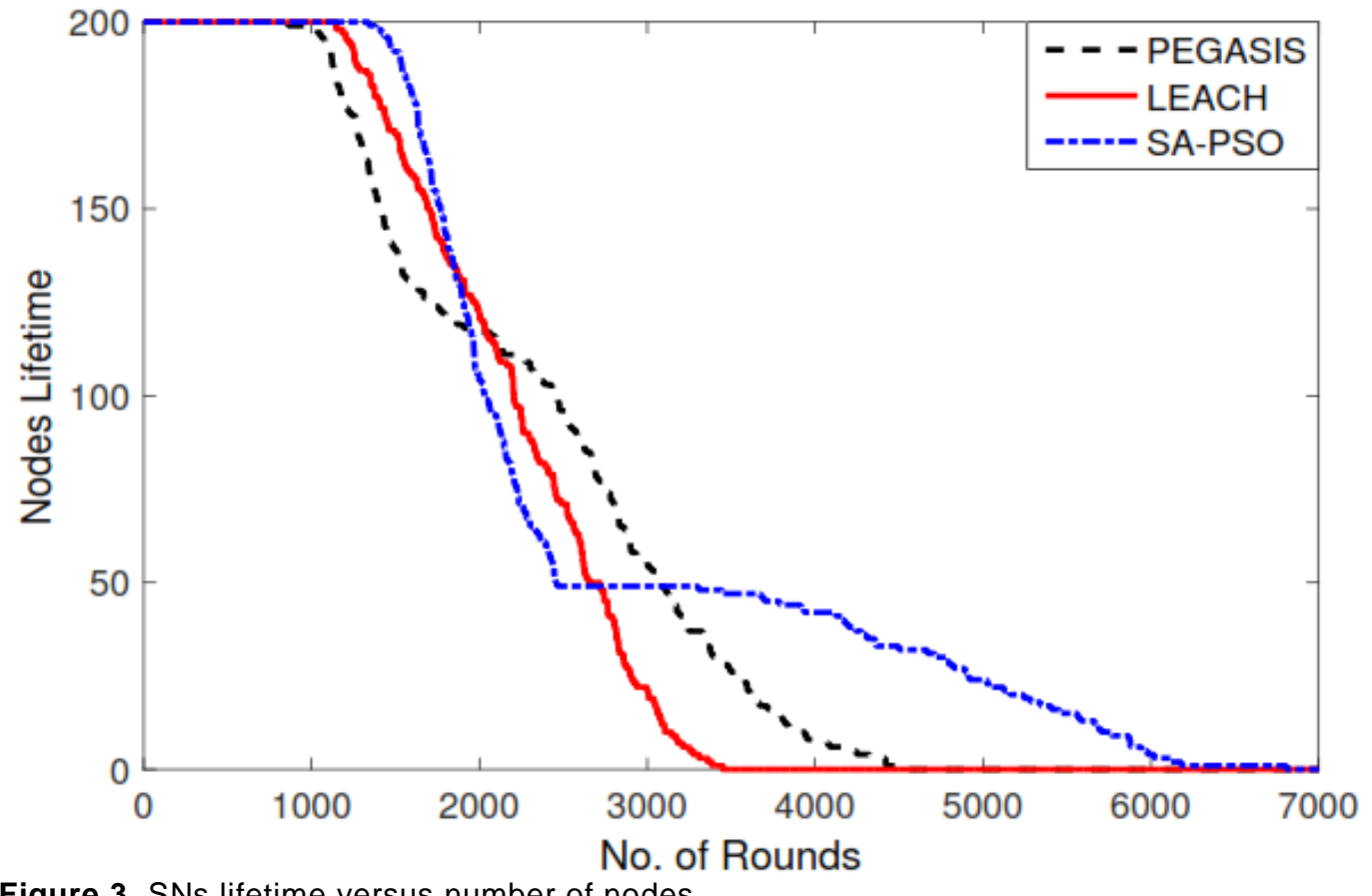

Figure 3. SNs lifetime versus number of nodes.

It is clearly seen in Fig. 3 that the lifetime of SNs in case of the proposed algorithm is more as compared to that of using LEACH and PEGASIS. It means that the performance of the network increases because of enhanced lifetime. From Fig. 3, it is seen that using SA-PSO, SNs die after completing more than 6000 rounds while for PEGASIS, nodes die out up to 4500 and for LEACH nodes die at 3500 . 


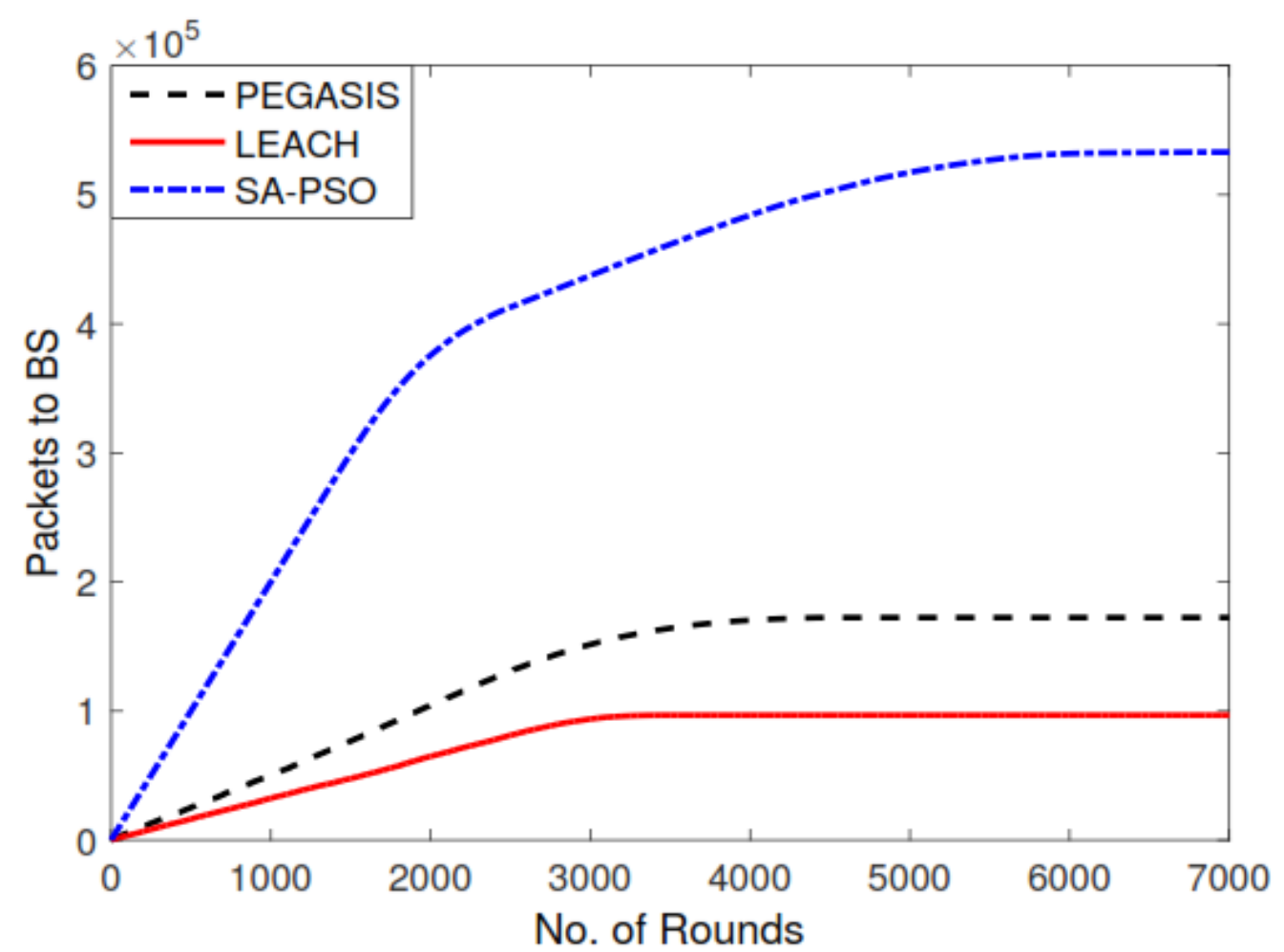

Figure 4. Data packet transmission to BS.

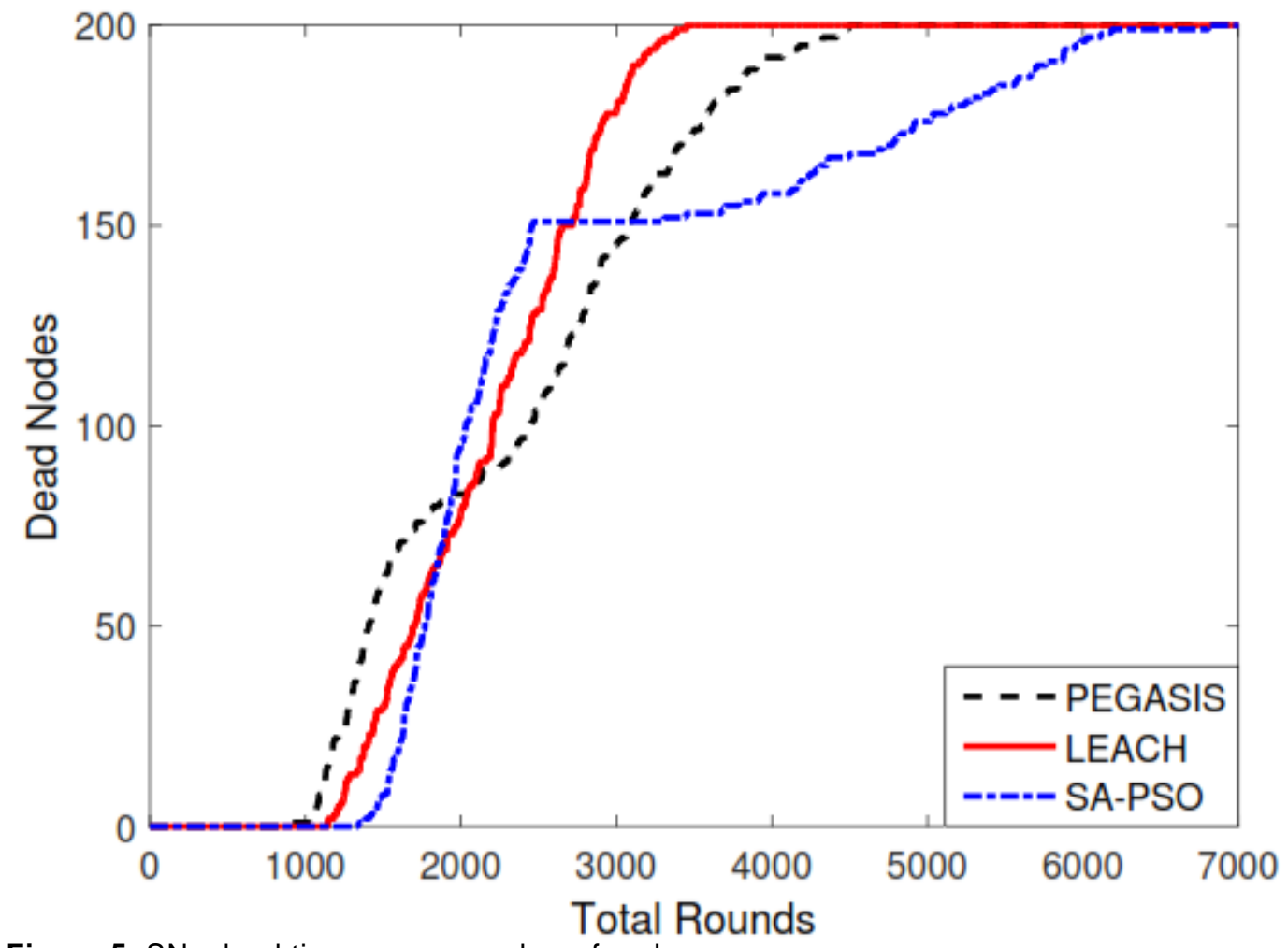

Figure 5. SNs dead time versus number of nodes.

Figure 4 presents the transmission of data packets to the BS. It is noted that SA-PSO outperform the other two LEACH and PEGASIS algorithms. It can be easily figured out that the data gathering efficiency is increased using the SA-PSO algorithm. Figure 5 shows dead nodes with the passage of time. It is 
seen that using SA-PSO, WSN has better performance because of longer lifetime.

For bigger WSNs, the low death rate of nodes not only reduces the network lifetime but its stability as well. After looking results, it is observed that the SA-PSO algorithm is comparatively more energy efficient algorithm for WSN rather than LEACH and PEGASIS in terms of lifetime and packet transmission as shown in Fig. 3 and Fig. 4.

\section{CONCLUSION}

In this research paper, an energy efficient scheme for data gathering in WSN is presented. A novel SA-PSO algorithm is proposed in which best chain formation procedure is adopted. The presented analysis shows that SA-PSO outperforms than PEGASIS and LEACH in terms of network lifetime, communication overhead and rate of $\mathrm{SNs}$ deaths. From simulation results, it is confirmed that SA-PSO outperforms LEACH and PEGASIS by eliminating the communication overhead in cluster formation and introduces low latency since it forms a chain among SNs. It is seen that SNs using SA-PSO remains active for over 1500 rounds than PEGASIS and 2500 rounds than LEACH.

\section{ACKNOWLEDGMENTS}

The authors would like to thank Dr. Ishtiaq Ahmad and Dr. Noor M. Sheikh for the follow discussion throughout the research work and thanks to Higher Education Commission Islamabad (HEC), Pakistan for their financial support to present the research work.

\section{REFERENCES}

S. Kalantary, and S. Taghipour, "A survey on architectures, protocols, applications, and management in wireless sensor networks", Journal of Advanced Computer Science and Technology, vol. 3, no. 1, (2014), pp. 1-11.

S. Gurbhej and K. Rajneet, "Review on Data dissemination and gathering in Wireless Sensor Networks", International Journal of emerging research in management and Technology, vol. 2013, no. 1, (2013), pp. 63--67.

S. A. Imam, V. K. Sachan, and V. P. Dubey, "Design and analysis of energy efficient communication methods for wireless sensor networks", Telecommunications and Radio Engineering, vol. 74, no. 19, (2015), pp. 1705--1714.

Y. Wu, Z. Mao, S. Fahmy, and N. B. Shroff, "Constructing maximum-lifetime data-gathering forests in sensor networks", IEEE/ACM Transactions on Networking, vol. 18, no. 5, (2010), pp. 1571--1584.

C. Karakus, A. B. Gurbuz, and B. Tavli, "Analysis of energy efficiency of compressive sensing in wireless sensor networks", IEEE Sensors Journal, vol. 13, no. 5, (2013), pp. 1999--2008.

Yongming Qin, Qiuling Tang, Ye Liang, Xiuyu Yue, Xian Li, "An EnergyEfficient Cooperative MIMO Scheme for Wireless Sensor Networks", 14th IEEE International Conference on Computational Science and Engineering. Dalian, China, (2011), pp. 471--474.

X. He, X. Zhou, M. Juntti, and T. Matsumoto, "Data and error rate bounds for binary data gathering wireless sensor networks", Proceedings of the IEEE 
16th International Workshop on Signal Processing Advances in Wireless Communications (SPAWC '15). Stockholm, Sweden, June (2015), pp. 505-509.

M. Radi, B. Dezfouli, K. A. Bakar and M. Lee, "Multipath Routing in Wireless Sensor Networks: Survey and Research Challenges”, Sensors, vol. 12, no. 1, (2012), pp. 650--685.

X. Xing, D. Xie, G. Wang, "Energy-balanced data gathering and aggregating in WSNs: a compressed sensing scheme", International Journal of Distributed Sensor Networks, vol. 10, no. 11, (2016), pp. 186.

K. Navdeep, S. Deepika, S. Prabhdeep, "Classification of Hierarchical Routing Protocols in Wireless Sensor Network: A Survey", International Journal of P2P Network Trends and Technology, vol. 3, no. 1, (2013), pp. 56--61.

M. Tong and M. Tang, "LEACH-B: An Improved LEACH Protocol for Wireless Sensor Network", 2010 6th International Conference on Wireless Communications Networking and Mobile Computing (WiCOM), Chengdu, China, Oct., (2010), pp. 1--4.

HU Sen-lai, ZHANG Yu, JIN Xin-yu, LI Hui-zhong, "Improvement of PEGASIS Algorithm in Wireless Sensor Networks Using GA", Journal of Jiangnan University, vol. 4, (2008), pp. 1-10.

J. Kennedy and R. C Eberhart, "Particle swarm optimization", Proc. IEEE International Conference on neural networks, IEEE service center, Piscataway, NJ, (1995), pp. 1942--1948.

O. Cakir, I. Kaya, A. Yazgan, O. Cakir, E. Tugcu, "Emitter Location Finding using Particle Swarm Optimization”, Radioengineering, vol. 23, no. 1, (2014), pp. 252--258.

Wen Liu, Zhiyuan Ma, Zhongliang Deng and Lianming Xu, "Energy Balance Algorithm based on LEACH", EEE 12th International Conference on Communication Technology, Nanjing, China, China, Nov., (2010), pp. 681 -684 .

N. Jasmine, J. J. Paulraj, and R. P. Prapoorna, "A Faster Routing Scheme for Stationary Wireless Sensor Networks - A Hybrid Approach”, International Journal of Ad Hoc, Sensor \& Ubiquitous Computing, vol. 1, no. 1, (2010), pp. 1--10.

M. T. Nguyen and K. A. Teague, "Tree-based energy-efficient data gathering in wireless sensor networks deploying compressive sensing”, Proceedings of the 23rd Wireless and Optical Communication Conference (WOCC'14). Newyark, NJ, USA, May (2014), pp. 1--6.

A. Norouzi, F.S. Babamir and A.H. Zaim, "A New Clustering Protocol for Wireless Sensor Networks using genetic algorithm approach", Wireless Sensor Networks, vol. 2011, no. 3, (2011), pp. 362--370.

M. Botta, M. Simek, "Adaptive Distance Estimation Based on RSSI in 802.15.4 Network”, Radioengineering Journal, vol. 22, no. 4, (2013), pp. 1162-1168.

S. K. Singh, P. Kumar and J. P. Singh, "A Survey on Successors of LEACH Protocol”, IEEE Access, vol. 5, (2017), pp. 4298--4328.

Mounir Arioua, Y. el Assari, Imad Ez-zazi and A. el Oualkadi, "Multi-hop Cluster Based Routing Approach for Wireless Sensor Networks", Procedia Computer Science, vol. 83, no. Supplement C, (2016), pp. 584--591.

N. G. Palan and B. V. Barbadekar and S. Patil, "Low energy adaptive clustering hierarchy (LEACH) protocol: A retrospective analysis", International 
Conference on Inventive Systems and Control (ICISC), Coimbatore, India, (2017), p. $\sim 1-12$.

Ez-Zazi, Imad, Arioua, Mounir, El Oualkadi, Ahmed and Lorenz, Pascal, "A Hybrid Adaptive Coding and Decoding Scheme for Multi-hop Wireless Sensor Networks", Wireless Personal Communication, vol. 94, no. 4, (2017), pp. 3017--3033.

M. N. Khan and M. Jamil, "Performance improvement in lifetime and throughput of LEACH protocol", Indian Journal of Science and Technology, vol. 9, no. 21, (2016), pp.1-6.

Muhammad Nasir Khan, "Importance of Noise Models in FSO Communications," EURASIP Journal of Wireless Communication and Networking (EURASIP, JWCN), vol. 2014, no. 102, Dec. (2014), pp. 1-10.

Muhammad Nasir Khan and Mohsin Jamil, "Maximizing Throughput of Free Space Communication Systems using puncturing Technique,” in Arab. J. Sci. \& Eng. vol. 39, no.12, (2014), pp. 8925-8933.

Muhammad Nasir Khan and M. Jamil. "EXIT chart behavior for the Hybrid FSO/RF communication system" in 2015 10th International Conference on Information, Communications and Signal Processing (ICICS), Singapore, (2015), pp. 1-5. 


\title{
DESIGN AND IMPLEMENTATION OF TCSC FOR 500KV POWER TRANSMISSION SYSTEM
}

\author{
Ali Raza \\ Department of Electrical Engineering, The University of Lahore, Lahore, \\ (Pakistan) \\ E-mail: ali.raza@ee.uol.edu.pk
}

Haroon Farooq Department of Electrical Engineering, University of Engineering \& Technology, Lahore, (Pakistan) E-mail: haroon.farooq@uet.edu.pk

Manzoor Ellahi Faculty of Engineering and Technology, Superior University, Lahore, (Pakistan) E-mail: manzoor.ellahi@superior.edu.pk

Waqas Ali Department of Electrical Engineering, University of Engineering \& Technology, Lahore, (Pakistan) E-mail: engr_waqasali@yahoo.com

Shahid Kaleem Department of Electrical Engineering, The University of Lahore, Lahore (Pakistan) E-mail: shahid.kalim@hotmail.com

Muhammad Nasir Khan Department of Electrical Engineering, The University of Lahore, Lahore (Pakistan) E-mail: muhammad.nasir@ee.uol.edu.pk 


\section{ABSTRACT}

Power transmission capability of a transmission line (TL) depends upon the impedance of the TL, the magnitude and the phase angle difference of the end voltages. Series capacitor largely employed in the transmission lines to increase the transfer capability but create instability problems. Flexible alternating current transmission systems (FACTs) enhance the power transfer through the existing transmission lines with stability intact. Thyristor controlled series compensation (TCSC) is considered in this paper. Impedance of the transmission line is regulated by changing the firing angle of the thyristor. A $500 \mathrm{kV}$ transmission line shunted with TCSC is dynamically implemented in Matlab/Simulink and tested for different sending end voltage and, by changing the impedance of line. Results show the significance of designed control under transient conditions of power system.

\section{KEYWORDS}

Flexible alternating current transmission systems (FACTS) devices; transmission lines; thyristor controlled series compensation (TCSC); stability enhancement.

\section{INTRODUCTION}

Energy is said to be a backbone of a nation's economy. With the passage of time, the world is becoming more automated and electronic and thus the consumption of electricity per person is increased. The human race is going to be more dependent on robots, thus it's important to fulfill the need of electricity. There are two ways to increase the transmission line (TL) capacity. In first choice, need to build new transmission lines to meet the demands. Installations of new transmission lines require feasibility studies, contract signing, electrical and mechanical designs and, material for wires. It is an extravagant choice and requires plenty of time for completion. Second option is to increase the power transfer capability of the existing transmission system. This method doesn't require any feasibility report and cost-effective as well.

A number of researches have been conducted for increasing the bulk power transfer capacity of existing transmission lines [1] - [3]. In early days, power flow control is done by changing taps or via phase shifting transformers. Series reactors were introduced in transmission lines to reduce the power flow and also used to reduce the short circuit current level at some locations when needed. Similarly, capacitors employed in transmission lines to reduce the electrical length and thus to increase the power flow. Hence, series compensation was used on alternate basis according to load condition. However, this kind of compensation introduced transient and stability issues [1]. So, an alternative technique is required to solve these shortcomings. Fixed series capacitors deployed in the transmission lines to increase the power transfer capacity. The introduction of series capacitor causes low frequency oscillations in the transmission system which introduced the effect of sub-synchronous resonance (SSR) in electric power system. Therefore, with the advent of power electronics devices, stated method is replaced by the flexible AC transmission system (FACT). FACTs controllers can not only increase the power transmission capability of the TLs but also offer advantages like damping of low frequency oscillations and mitigation of SSR damping's etc.

IEEE defines FACTS technology as a power electronic system that is used in AC transmission system to enhance controllability and thus increase the power 
transfer capability [2], [3]. The FACTs controllers are categorized according to their generations. Static VAR compensators (SVC), thyristor controlled phase shifters (TCPS) and thyristor controlled series capacitors (TCSC) are known as first generation FACTS controllers. TCSC used to control the impedance of the TL, where a silicon rectifier connected in series combination with a capacitor [4]. In this research, a dynamic TCSC model for $500 \mathrm{kV}$ transmission line is implemented in Matlab/Simulink and tested with two checks: by changing impedance of TL, and by varying the sending end voltage.

The manuscript is organized as follows; effect of capacitors on power transfer when employed in transmission lines is explained in section II. In section III, detailed working of TCSC and employed capacitive mode is described. Section IV and V deal with simulations and results. Finally, conclusions are drawn in section VI.

\section{EFFECT OF CAPACITORS ON POWER TRANSFER IN TRANSMISSION} LINES

The steady state power transfer capability of transmission line is explained through two machine power system model as shown in Figure 1 [5]. Power is transmitted from one terminal (sending end) bus to second terminal (receiving end). Mathematical formulation for power transfer capability is given by:

$$
P_{c}=\frac{V_{s} V_{r}}{X_{L}} \operatorname{Sin} \delta
$$

Where Vs and Vr are sending and receiving ends voltages, respectively, and XL is the indicative impedance of transmission line. $\delta$ represents the phase angle between sending and receiving end voltages. Thus, the power transfer capability of TL largely

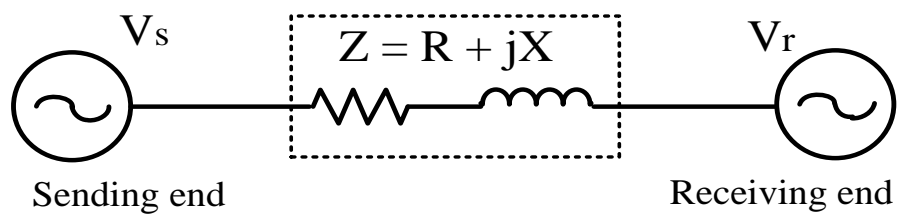

Figure 1. Two machine transmission system.

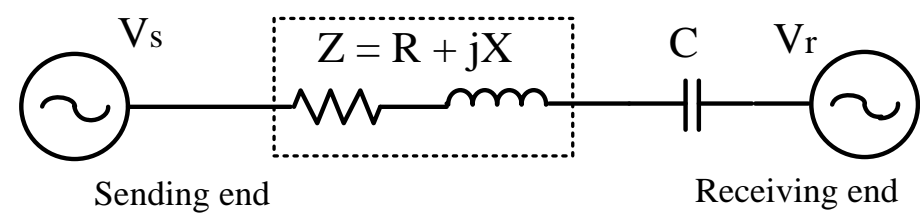

Figure 2. Two machine transmission system with series capacitor.

depends upon the magnitude of the sending and receiving end voltages, phase angle between them and inductance of line.

Conversely, a series capacitor is inserted in transmission system to study the power transfer capability as shown in Figure 2 [5]. Ability of power transmission is increased with such an insertion of capacitors as series capacitors cancel the inductance of transmission line and boost the power flow as:

$$
P_{c}=\frac{V_{s} V_{r}}{X_{L}-X_{C}} \operatorname{Sin} \delta
$$


Where Xc is the capacitive impedance of series capacitors. Due to mechanical switching of series capacitors, transients produced and only fixed value of series compensation achieved [5].

\section{THYRISTOR CONTROLLED SERIES CAPACITOR}

Thyristor controlled series capacitor is a FACTs controller. Mainly, TCSC devices are employed in the series of TLs to increase the power flow. Impedance of TL is regulated by controlling the firing angle of the thyristors. Practically, more than one TCSCs are installed in TL [6]. A TCSC consists of a fixed capacitor which is shunted by a thyristor controlled reactor (TCR) as schematically drawn in Figure 3 [7].

A TCR consists of an anti-parallel thyristors and inductor in series. With TCSC, flexible compensation is achieved because the gate terminal of thyristor is triggered at various firing angles to insert different values of capacitor in series of transmission line. $75 \%$ compensation is achieved, in this paper, by using TCSC. Normally, a TCSC has three modes of operation:

1. Thyristor blocked

2. Thyristor bypassed

3. Vernier operation

In thyristor block mode, thyristors are not conducting and the value of $\alpha$ is 1800 . The effective impedance of TCSC is only because of capacitive reactance of capacitor. In thyristor bypass mode, valves are gated for full conduction and capacitor is bypassed. Practically, some current flows though capacitor but it is negligible. Vernier operation is further categorized into capacitive and inductive mode as shown in Figure 4 [8].

A TCSC operates in inductive region when there is no load condition in power system [9]. Under this mode, TCSC behaves as a source of inductive reactance which decreases the power transfer capability of transmission line. However practically, very rare chances of no load condition in electric power system that is why TCSC is not employed in this scenario. In capacitive mode, TCSC behaves as a source of capacitive reactance to cancel the inductive reactance of TL and thus, increases power transmission capacity of existing transmission line [10]. Practically, power systems are being operated at overload condition due to which, generally TCSC is employed in capacitive mode. TCSC in not operated in resonance region. TCSC can operate within range from $180 \mathrm{o}$ to alpha minimum for capacitive region. If TCSC is allowed to operate at an angle of $180 \mathrm{o}$, no current flows through TCR and the effective impedance of the TCSC is due to the capacitor [11]. If the value of alpha is somewhat between $180 \mathrm{o}$ to alpha minimum, then TCSC reactance is greater than the Xc. The resonance point is reached when $\mathrm{Xc}$ is equal to TCR XL. Required compensation achieved, in capacitive mode, with firing angle range of $690-900$ and $163 \mathrm{~Hz}$ oscillatory frequency, which is 2.7 times the $50 \mathrm{~Hz}$ [12]. Impedance of TL is at the lowest level at 900, that is power transfer capability reduces as the firing angle increases. Impedance is about $120-136 \mathrm{Ohm}$ at time of capacitive mode of TCSC. 


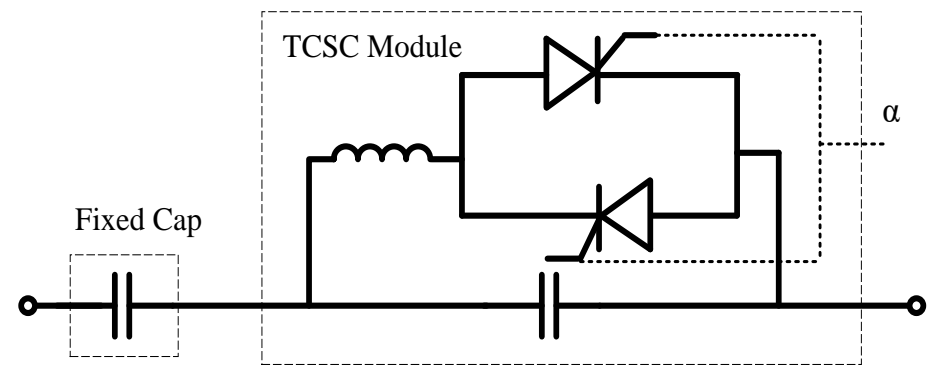

Figure 3. Thyristor controlled series capacitor module.

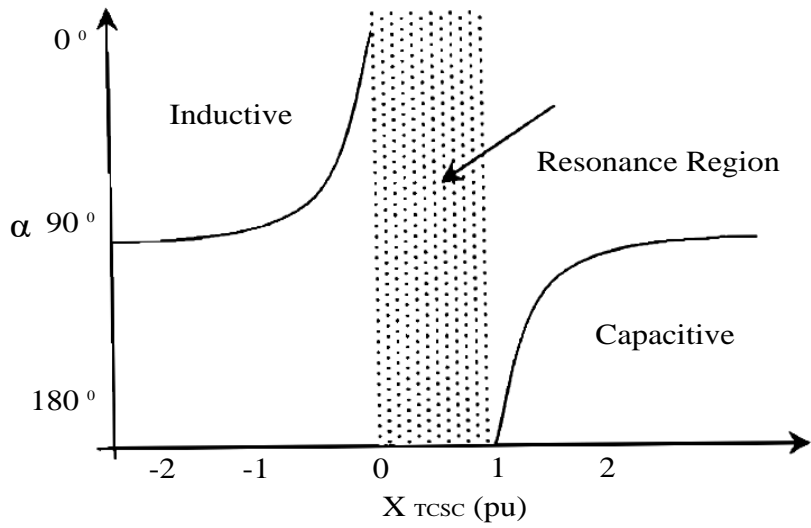

Figure 4. TCSC characteristics.

\section{SIMULINK MODEL OF TCSC FOR 500KV TRANSMISSION LINE}

Mathematical model of TCSC is developed for $500 \mathrm{kV}$ three phase primary transmission line. One TCSC compensates single phase of transmission line, thus for three phases three block of TCSCs are developed. Net reactance of TCSCs is regulated by changing the firing angle of the thyristor. Angle alpha is synchronized with the line current by using the phase lock loop (PLL). Proportional integral (PI) controller is used for the feedback purpose. The developed model includes sending and receiving ends AC voltage sources, TCSC block and the controller. The controller of TCSC includes control unit [12] and firing unit [13] as shown in Figure 5. System under study consists of a programmable voltage source at sending and receiving ends of TL. The purpose of using the programmable voltage source is to vary the voltage at different time instants.

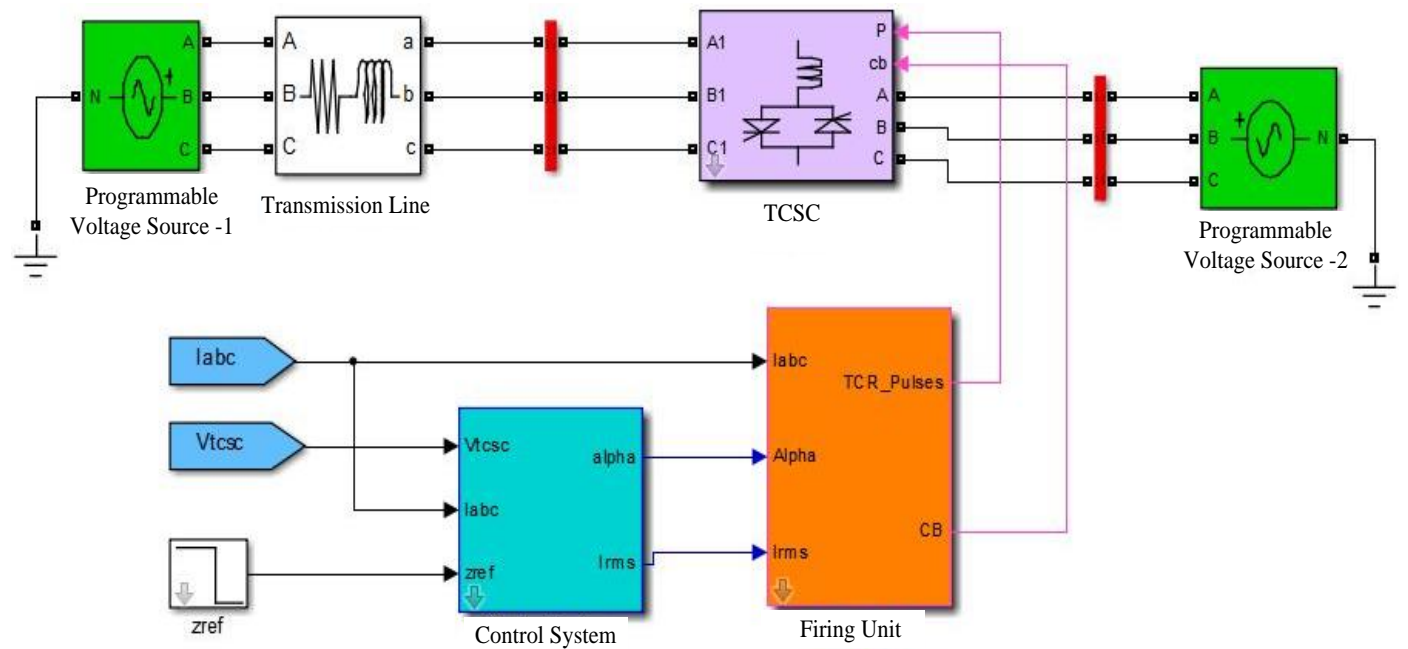

Figure 5. Simulink model of TCSC emplyed within transmission system for $75 \%$ compensation. 


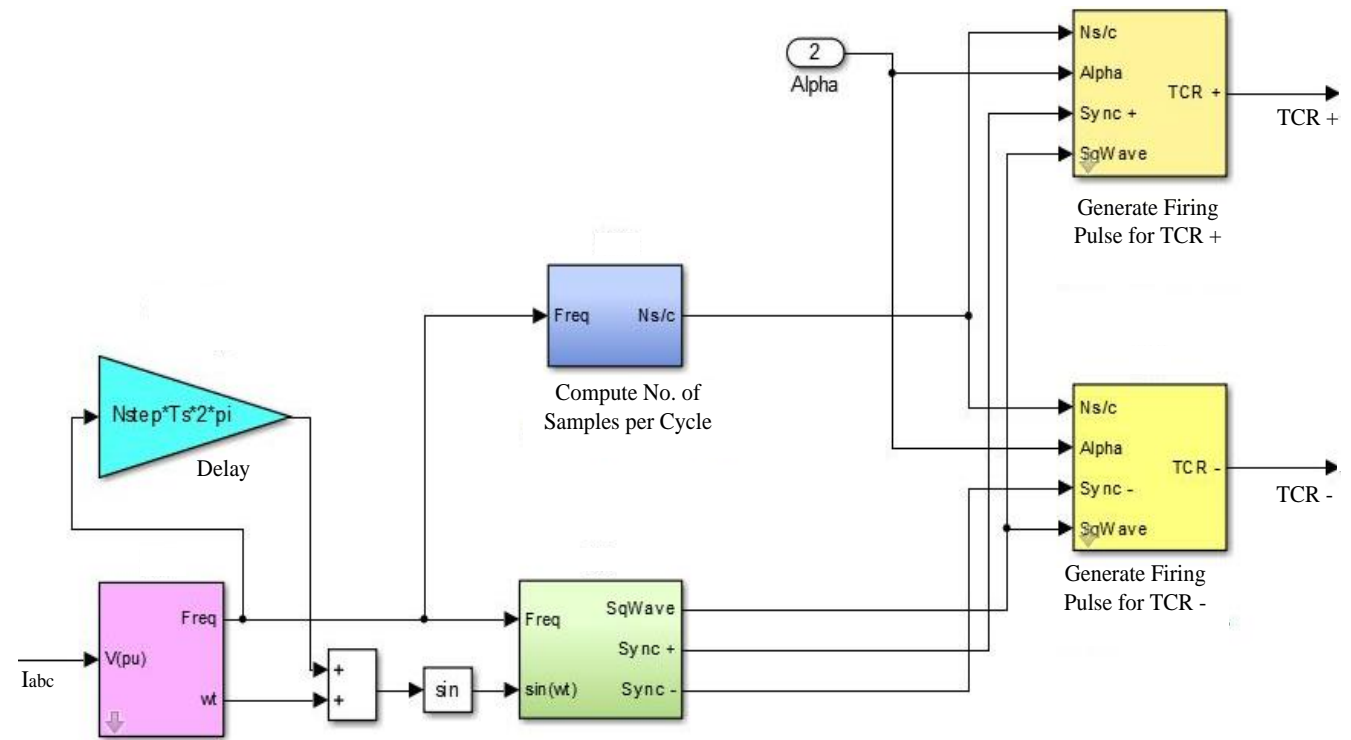

Figure 6. Simulink model of firing unit for single phase.

The series line compensation block consists of TCSC, controller, three phase voltage measurement and scopes for measurement. Triggering pulses for TCSCs are controlled through controller block which consists of control and firing units. Control unit calculate the firing angle based upon the impedance. Initially, the impedance is calculated using RMS values of voltage and current by applying ohm's law, marked as measured impedance. To remove the second order harmonics, it is passed to second order filter [14]. Error is calculated by taking difference of the measured and reference impedances [15]. After that the values are passed through the proportional integral controllers.

In firing unit, line current is synchronized using PLL and then compared and, zero crossing is checked of the line current to generate square wave and to synchronize the pulses as shown in Figure 6. Synchronization pulses are triggered at the start of positive and negative cycles and square wave indicates the duration of a cycle. And then by using discrete time integrator, square wave converted into the saw tooth wave. Rounded method is used to convert alpha into a single value for the comparison of alpha with saw tooth wave. Finally, compared saw tooth wave with alpha value generate triggering pulse for thyristor gate.

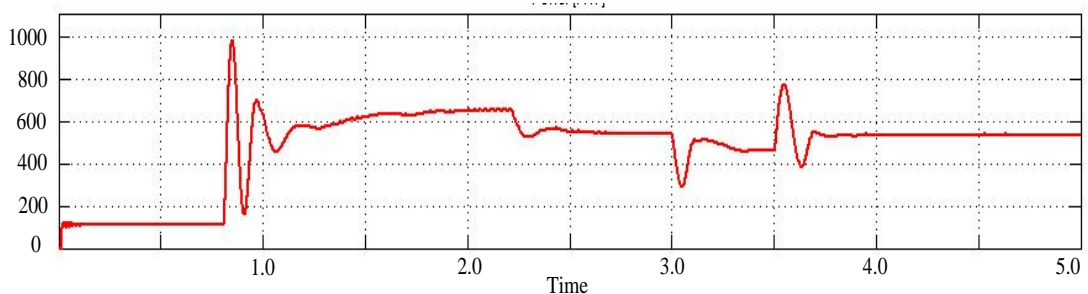

a) Transmitted power via 500kV transmission line

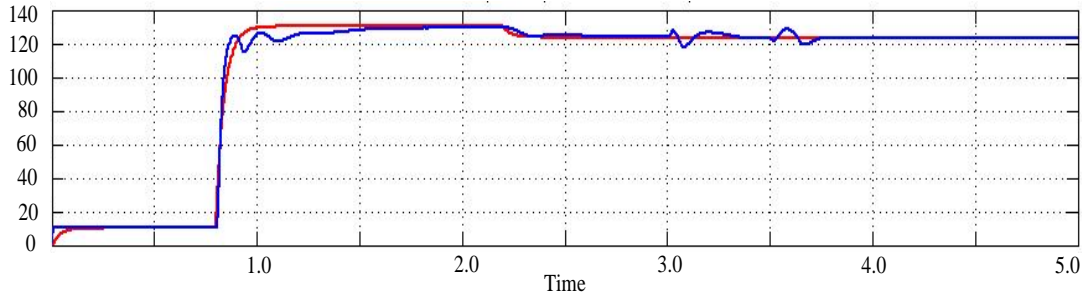

b) Impedance of 500kV transmission line 


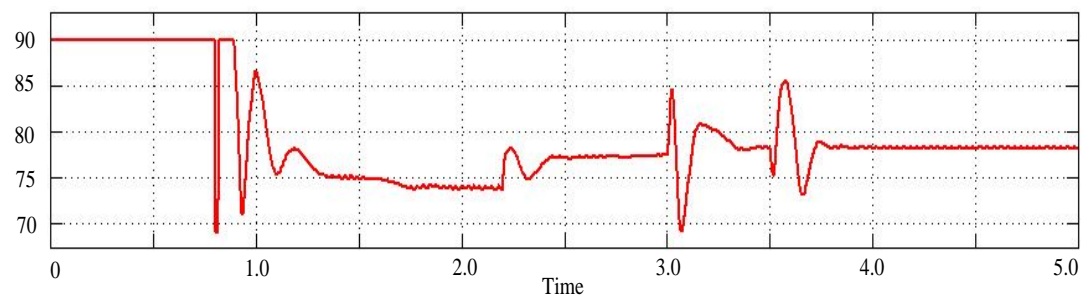

c) Firing angle of TCSC in series with $500 \mathrm{kV}$ transmission line

Figure 7. Transmitted power, impedance of transmission line and firing angle of TCSC inserted in a two machine power system.

\section{RESULTS AND OBSERVATIONS}

Transmission system is designed for $500 \mathrm{kV}$ but due to losses in the lines, receiving end voltages reduced to $477 \mathrm{kV}$. Power, impedance and firing angle profiles of the proposed system are shown in Fig. 7. $115 \mathrm{MW}$ is the power transfer before triggering of TCSC. TCSC operates in capacitive mode and triggered at $0.8 \mathrm{sec}$, power transfer increased to $650 \mathrm{MW}$ and measured impedance follow the reference impedance as shown in Figure 7 (a) and (b), respectively.

$5 \%$ change in reference impedance is applied at $2.25 \mathrm{sec}$ and the response shows that TCSC successfully trace the reference impedance within around $400 \mathrm{~ms}$ as shown in Figures 7 . At $3.0 \mathrm{sec}, 5 \%$ reduction in sending end voltage is introduced and corresponding change in impedance is shown in Figure 7 (b). TCSC tries to match with the reference impedance by compensating the disturbances and lowering the power transfer to $500 \mathrm{MW}$.

Vs returned to 1p.u. at 3.5sec. Corresponding autonomous adjustments of power, firing angle and impedance of system are shown in their respective Figure 7 (a), (b) and (c), respectively.

It is observed that when the voltage drop occurs from programmable voltage source, the TCSC immediately respond to the power oscillation and damp it out but the power transfer does not remain constant before and after the disturbance. Power change from 530MW to 460MW during oscillations due to voltage reduction and returned to original value $(530 \mathrm{MW})$ whence the Vs $=1$ p.u. as indicated in Figure 7 (b).

\section{CONCLUSIONS}

Interconnected power system is employed using $500 \mathrm{kV}$ transmission line. TCSC is used to increase the power transfer capability of transmission system than constructing new lines. A dynamic simulation developed in Matlab/Simulink and tested for with and without TCSC, different sending end voltages, and by changing the impedance of transmission line. Reactive power demand of power system is compensated through TCSC. Results show that voltage regulation is improved, SSR mitigated and more stabled electric power system is achieved. Simulations reveal that installation of TCSC at high tension lines shows better performance. 


\section{ACKNOWLEDGMENT}

The authors are grateful to Prof. Xu Dianguo, IEEE Fellow and Prof. W. Barry Williams for thorough discussion during the research work and thanks to Punjab Higher Education Commission (PHEC) Pakistan for providing financial support to present this research work on international forum.

\section{REFERENCES}

[1] Jalali S. G., Lasseter R. H. and Dobson I. Dynamic Response of a Thyristor Controlled Switched Capacitor. IEEE Transactions on Power Delivery 1994; 9(3):1609-1615.

[2] Discover the World of FACTS Technology - Technical Compendium, Available at: http://www.energy.siemens.com/co/pool/hq/powertransmission/FACTS/FACTS _Technology_.pdf. Accessed on 10 Jan. 2017.

[3] Asare P., Diez T., Galli A., O'Neill-Carillo E., Robertson J., Zhao R. An Overview of Flexible AC Transmission Systems. Electrical and Computer (ECE) technical report, Purdue University; 1994.

[4] Acharya N., Arthit, Yome S., Mithulananthan. Facts about Flexible AC Transmission Systems (FACTS) Controllers: Practical Installations and Benefits. Proceedings of Australian Universities Power Engineering Conference (AUPEC); 2005 Sep 25-28; Hobart, Tasmania, Australia

[5] Deng Y. Reactive Power Compensation of Transmission Lines [MASc thesis]. Montreal, Canada: Concordia University; 2007.

[6] Maruf M, Mohsin A. H. N, Shoeb MD. A, Islam MD. K, Hossain MD. M. "Study of Thyrister Controlled Series Capacitor (TCSC) as a useful FACTS Device. International Journal of Engineering Science and Technology 2010; 2(9).

[7] Yarlagadda V, Sankar B.V, Rao K. R. Automatic Control of Thyristor Controlled Series Capacitor (TCSC). International Journal of Engineering Research and Applications 2012; 2(3): 444- 449.

[8] Rashid H. M. Power Electronics: Circuit Devices and Application. Third edition. Prentice Hall; 1993.

[9] ABB to Enhance 400kV Raipur-Rourkela Transmission Line with State-of-the-Art Flexible AC Solution. Bangalore India: ABB; March, 2003. 02 p.

[10] IEEE Recommended Practice for Specifying Thyristor-Controlled Series Capacitors. IEEE Std. 1534-2009 (Revision of IEEE Std 1534-2002). IEEE; Nov. 202009.98 p.

[11] Mazibuko, Ronnie H. Design and Implementation of a Thyrister Controlled Series Capacitor for Research Laboratory Application [M.Sc. thesis]. South Africa; University of Natal, Durban; 2003.

[12] Jovcic D., Pillai G. N. Analytical modeling of TCSC dynamics. IEEE Transactions on Power Delivery 2005, April; 20(2):1097-1104.

[13] Sen T., Bhattacharjee P. K., Bhattacharya M. Design and Implementation of Firing Circuit for Single-Phase Converter. International Journal of Computer and Electrical Engineering 2005, June; 3(3):368-374. 
[14] TCSC (phasor model) [Internet]. 2016 [cited 2017 Jan 7]. Available from: https://www.mathworks.com/help/physmod/sps/examples/tcsc-phasormodel.html.

[15] Kumkratug, Prechanon. The Mathematical Model and Simulation of Static VAR Compensator in Medium Transmission Line. American Journal of Applied Sciences 2012, May; 9(5). 
Design and implementation of TCSC for 500KV power transmission system

DOI: http://dx.doi.org/10.17993/3ctecno.2019.specialissue.13 


\section{ARDUINO BASED RADAR SYSTEM}

Sarmad Hameed

Mechatronics department, Shaheed Zulfikar Ali Bhutto Institute of Science and Technology, Karachi, (Pakistan) E-mail: sarmad.hameed@szabist.edu.pk

Naqi Jafri Mechatronics department, Shaheed Zulfikar Ali Bhutto Institute of Science and Technology, Karachi, (Pakistan) E-mail: Naqijafri10@gmail.com

Dania Rashid

Mechatronics department, Shaheed Zulfikar Ali Bhutto Institute of Science and Technology, Karachi, (Pakistan) E-mail: daniarashid118@gmail.com

Fabiha Shoaib

Mechatronics department, Shaheed Zulfikar Ali Bhutto Institute of Science and Technology, Karachi, (Pakistan) E-mail: fabihashoaib@hotmail.com 


\section{ABSTRACT}

This paper is about Radar System controlled via Arduino. This RADAR system consists of an ultra-sonic sensor and servo motor, these are the major components of the system. Basic working of the system is that it have to detect objects in its defined range. Ultra-sonic sensor is attached to the servo motor it rotates about 180 degree and gives visual representation on the software called processing IDE. Processing IDE gives graphical representation and it also gives angle or position of the object and distance of the object. This system is controlled through Arduino. Arduino UNO board is sufficed to control ultrasonic sensor and also to interface the sensor and display device. While researching, we learned about existing navigation and obstacle detection innovations and different systems where ultrasonic sensors are used efficiently. Main application of this RADAR system comes into different field of navigation, positioning, object identification, mapping, spying or tracking and different applications. These less investment system are also suitable for indoor applications.

\section{KEYWORDS}

Arduino, ultra-sonic, radar, positioning, surveillance, obstacle detection.

\section{INTRODUCTION}

RADAR system is an object detection or tracking system which uses radio waves to decide or get the range, height, heading, or speed of items or objects. Radar frameworks or system arrive in an assortment of sizes and have distinctive performance particulars. Some radars are utilized for aviation authority at air terminals and others are utilized for long range observation and earlycautioning frameworks [1]. There are some ways to show radar working data. There are also some modified radar systems which have advance technology of handling the systems. These modified system are used at higher levels to get or extract the helpful or important data [2].

Our proposed system's working principle is linked by the following components which are is ultra-sonic sensor connected to the microcontroller (we have chosen Arduino) digital input and output pins. Then we have servo motor which is also connected to digital output and input pins. Our both main components ultra-sonic sensor and servo motor are connected simultaneously, so that when our servo motor rotates from 0 degree to 180 degree from extreme right to extreme left the motor will rotate nearby its axis [3]. We utilize Computer screen to demonstrate the data (distance and angle) through software called "Processing development Environment" [1].

\section{LITERATURE REVIEW}

Subsequent to experiencing a portion of the papers with respect to usage utilizing ultrasonic sensors and ARDUINO, it was found that this idea is searched a lot and is a mainstream idea which is still in advance. The advances utilized were not just productive and solid yet in addition financially achievable 
[5]. Not only this, here other very useful applications of ultrasonic sensors were observed too.

This paper discusses about a monitoring system which is designed measure to speed of waves and height of river through ultra-sonic sensor using microcontroller (Arduino). On the off chance that the waterway can't oblige the volume of water, then all the water will submerge with land and this phenomenon is called as flood or surge. We can overcome this flood problem by earlier identification in height of water and observing speed. If we identify problem earlier we can overcome this problem before it become crisis. By testing the system i.e. simple water level, it was observed that ultra-sonic have accuracy of $96.6 \%$. But when it is implemented in the rivers there are many errors because of different type of water levels due to heavy waves and speed of water and also due to floating of heavy objects. Unlike Previous testing results, author directed this analysis on tracking of speed of water improvement or modification and level of water in flooding. The test was completed when the Arduino used as controller of application. For more research, information of depth level and speed of water of this system will be sent to database server website to be checked regularly [8].

An intelligent driver monitoring and vehicle control system is introduced in this research. This technology is create to avoid accidents by monitoring the driver's activities. The writer states some of the main reasons of accidents today. These are alcohol consumption by the driver, carelessness, drowsiness or medical illness. The various units in the framework, including motors, relays, power unit and ESP8299 module are tried and are observed to be in working condition. Ultrasonic sensor is utilized to alarm the driver if any vehicle draws close to his vehicle. The status of the driver can be observed by the assistance of sensors executed in the vehicle and the subtle elements are refreshed to the proprietor. This system overcomes all the different aspects due to which other technologies designed for this purpose have failed, making the system more useful, efficient and less costly and less time consuming [7]. In this research paper authors have given information about the detection of radio waves and tracking or ranging through radar set which is built from components like an ultra-sonic sensor, a servo motor and an Arduino. The author discuss about the linear measurement problem because of which distance measurement was not possible between some objects, was resolved with the introduction of Ultrasonic distance measurer. It allows to take noncontact measurements. This radar system can drastically reduce power consumption. The author says, that this system is an extremely handy radar system, it can read or track the distance and angle of an obstacle and shown it up on the monitor screen. The ultra-sonic was attached on top of the servomotor to detect obstacles at 0 degree to 180 degree from right to left. Both the ultra-sonic sensor and the servo were fueled and controlled by the Arduino controller. The GUI was built using the JAVA programming language to show the result on the monitor [3].

This paper represents a system for obstacle detection in a known environment. This system works through an android based mobile camera. People who are 
visually impaired, face difficulties in detecting obstacles and navigation while they walk. They use sticks for this problem nonetheless this manner or technique is not right way of doing it. Object indicator or detector can overcome accidents or collision problems of people or the other way is they can to accurate map reading. The algorithm which is proposed in this paper is made for indoor mapping. In indoor surrounding all distinctive floors are taken in consideration and single image is kept or stored for distinctive floors. These images of floor are taken as reference image. The author mentions that this algorithm is $96 \%$ accurate and works in real time. There are different techniques discussed in this paper for obstacle detection. For these types of problems we can use the approach of SONAR sensor and also laser camera .In this paper introduced a calculation for identifying hindrance in known condition with an android based versatile camera which scans chosen territory before the camera for impediment location [9].

This research is about a blind walking stick made for blind people through which they can avoid obstacles while they walk and recognize currency. With the thought of visually impaired individuals, it is to some degree troublesome job to distinguish the cash or any unexpected obstacle. Despite the fact that currency dependent on size could possibly be recognized however it is relatively hard to distinguish that whether the note is unique or phony. So to overcome this issue the authors have designed the Currency Recognition Blind Walking Stick. A lot of work is done on currency recognition and obstacle detection using advanced technologies like optical character recognition, SURF and pattern extraction through colors. But none of these systems had the feature for obstacle detection for blind people. Therefore this framework is efficient as the other ones having an extra feature for helping the visually impaired [10].

\section{METHODOLOGY}

In order to testify the working of this system, after its designing, construction and programming we placed few objects in front of the ultrasonic sensor. As the motor started to rotate, our monitor started to display the output through processing IDE. Hence, when the sensor crossed over the object it showed a red segment with the distance and angle where the object is paced.

The first object was placed at the distance of $30.5 \mathrm{~cm}$ measured through a ruler and the system measured the distance at $32 \mathrm{~cm}$. While the second object was placed at a distance of $20 \mathrm{~cm}$ and the system measured it as $21 \mathrm{~cm}$. Hence the calculated efficiency turned out to be $95 \%$.

\section{System Overview:}




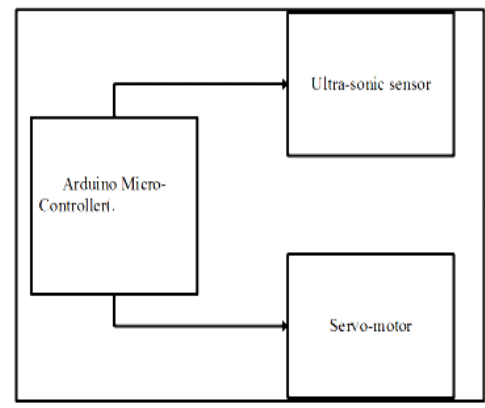

Figure 1. System hardware description.

The above figure represents a brief overview of this radar system. Here, as it is shown the controller we are using is Arduino, with the input Ultrasonic sensor and the output is the servo motor which rotates 180 degrees. The microcontroller controls all the operations of this system, from rotation of the motors to the obstacle detection of the ultrasonic and representation of the result on the screen.

\section{System Block Diagram:}

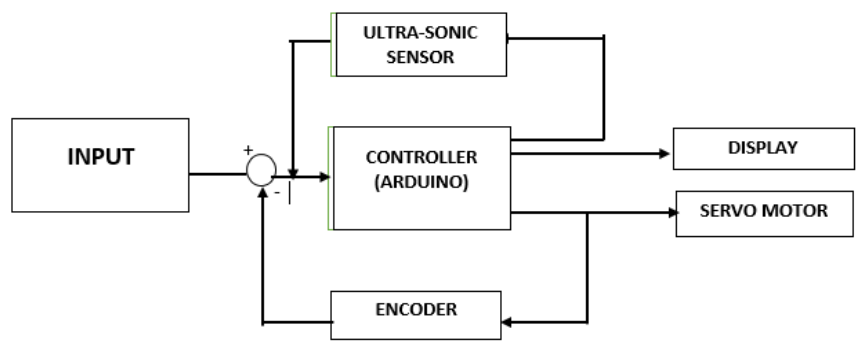

Figure 2. Block Diagram of Radar System.

Figure 2 represents the system's block diagram.

Here, it can be seen how the work flow in this radar system. The sensor is going to sense the obstacle and determine the angle of incident and its distance from the radar. The servo motor is constantly rotating to and fro, hence making the sensor move. The data obtained is encoded and fed to the processing IDE which represents it on the screen. The results are displayed further in this paper. All these operation are done by Arduino microcontroller from the rotation of the servo, data collection from the sensor, feeding the data to encoder to transferring it to the display.

\section{WORKING}

The basic objective of our design is to ascertain the distance position and speed of the obstacle set at some distance from the sensor. Ultrasonic sensor sends the ultrasonic wave in various ways by rotating with help of servo motors. This wave goes in air and gets reflected back subsequent to striking some object. This wave is again detected by the sensor and its qualities is analyzed and output is shown in screen indicating parameters, for example, distance and position of object. Arduino IDE is utilized to compose code and transfer coding in Arduino and causes us to detect position or angle of servo motor and it is communicated through the serial port alongside the covered distance of the nearest object in its way. Output of all of this working is shown in the software 
called processing, it will display the input/output and the range of the object [4]. Implementations of the sensors are done in such a way that ultra-sonic sensor is attached on top of the servo motor because it have to detect the object and its distance. Arduino (micro-controller) will control the ultra-sonic sensor and servo motor and also powered will be given to both of them through micro-controller [3].

Figure 3. Flow chart of Radar System.

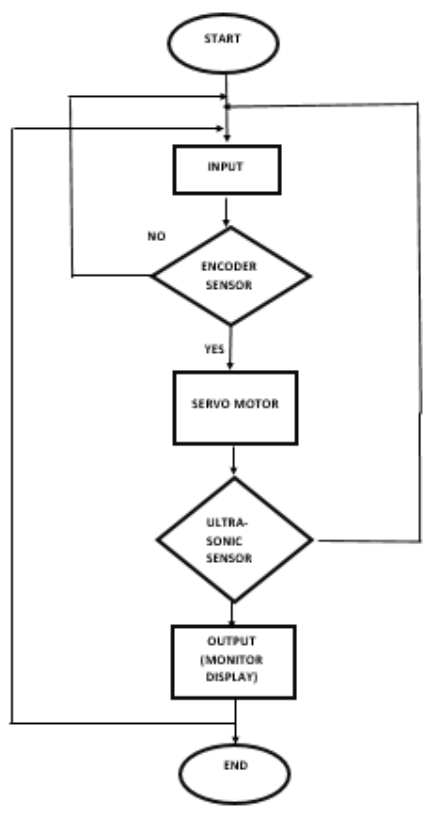

The above flow chart explains the working and the decision flow of this framework. As it can be seen the system starts with an input i.e. when the ultrasonic sensor detects an object, or does not detects any object, at any condition the encoder feeds the information in the controller while the servo keeps constantly rotating. As soon as any obstacle/objet is detected by the ultrasonic sensor the data is immediately processed by the controller and is fed to the IDE which shows it on the display screen. Here the process ends with an estimated distance of the object from the system with the angle at which it is placed.

\section{DISCUSSION AND RESULT}

In this research paper we have mentioned that our system is designed consisting following components such as, a servo- motor, an ultra-sonic sensor and a micro-controller (Arduino). System's objective is to track the distance and angle of the object and to represent this information graphically, means its output should be in graphical form which will be represented through processing software. We can have an idea of an efficiency of this radar by testing objects at different levels and observe how faster or smoothly it detects an object that it finds in a way and gives us an expected range of the obstacle [3].

Following figure show the results of the monitor screen of our design when the sensor rotates through the area and detects obstacle in the way. The red area indicates the presence of obstacle and below the angle of incident and distance is being displayed. 


\section{Testing of the system}

a) Object 1 is placed 30.5 far from the radar, radar gives the distance 32 $\mathrm{cm}$, so:

$$
\begin{array}{ll}
\circ & \text { error }=(32-30.5) / 30.5) * 100=4.918 \% \\
\circ & \text { efficiency } 1=100 \text {-error }=95.08 \%
\end{array}
$$

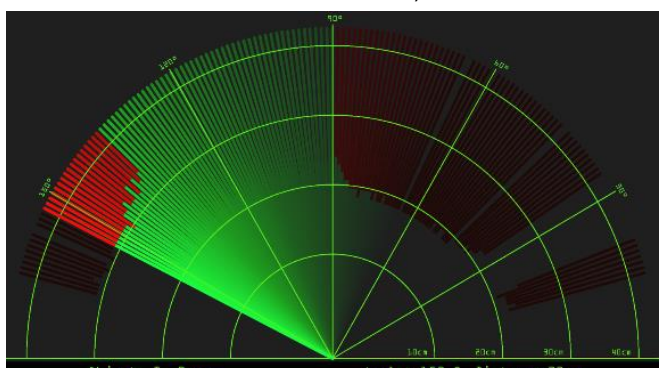

Figure 4. Processing IDE Screen displaying output of the system which we teseted by placing objects.

b) object 2 placed at a distance of $20.3 \mathrm{~cm}$,radar gives the distance $21 \mathrm{~cm}$ so:

○ $\quad$ error $=((21-20.3) / 20.3) * 100=3.44 \%$

○ efficiency $2=100$-error $=96.55 \%$

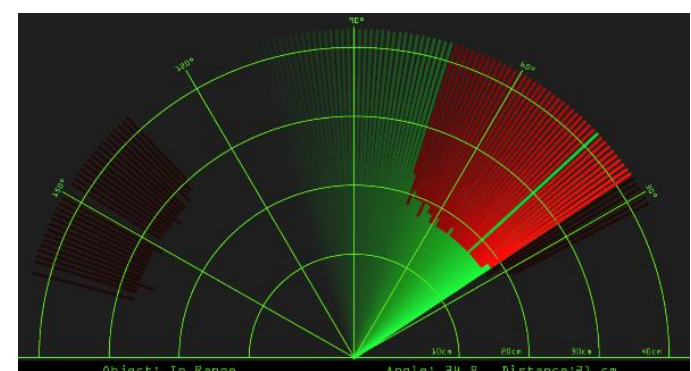

Figure 5. Processing IDE Screen displaying output of the system which we teseted by placing objects.

After the observations and calculations we can conclude that this system is $95.815 \%$ efficient.

\section{APPLICATION}

This Radar System have various applications for security purposes and it is mainly used for mapping.

- APPLCATION IN AIR FORCE:

It is used in airplanes or aircraft machines which have implemented radar system in it to detect the objects that comes in a way. It is also used to calculate height readings.

- APPLICATION IN MARINE:

This radar system also used in ships or marine. It is implemented on big ships to calculate the distance of other boats or ships, with the help of this sea accidents can also be reduced by not colliding. It can also be implemented on ports to see the distance of other ships and to monitor or control the ship movements.

- APPLICATON IN METEROLOGY: 
Meteorologists also uses radar systems to track or monitor the wind. It has been become an important equipment for climate testing. For example to detect tornados, storms.

\section{CONCLUSION}

Numerous advanced control methods gave designers to have more command over different advanced applications. In our paper, the recommended mapping method of whole system is assessed on small principles or scale [11]. The field that we have chosen for our design "Radar System" is a very vast field and future scope of this technology is very high. We have tremendous applications in which radar system have been implemented or used [3]. There is a lot of future scope of this design because of its security capacity. It can be used in many applications. This framework can also be developed or modified according to the rising needs and demand [4].

As we have designed a short range radar therefore our research was specified and limited. This system can only detect objects from 0 to 180 degrees only because the servo motor that we have used can rotate only to this range. So, due to this limitation our design cannot be applied to places or areas for obstacle detection on a larger scale. Usage of a 360 degrees rotating servo motor can make the system more efficient. We look forward to modify this system and enhance our research work by using a fully 360 degrees rotating servo and a higher ranged ultrasonic sensor. We can further add features to this system i.e. making it mobile, mounting an alarm system to it which turns on when obstacle is detected. Further modifications could be an obstacle avoiding robot with surveillance system.

\section{REFERENCES}

[1] Ahman Emmanuel Onoja, Abdusalaam Maryam Oluwadamilola, Lukman Adewale AJAO-"Embedded System Based Radio Detection and Ranging (RADAR) System Using Arduino and Ultra-Sonic Sensor” American Journal of Embedded Systems and Applications 2017

[2] Shreyes Mehta, Shashank Tiwari-"RADAR SYSTEM USING ARDUINO AND ULTRASONIC SENSOR” IJNRD, Volume 3, Issue 4 April 2018

[3] Antonio Tedeschi ; Stefano Calcaterra , Francesco Benedetto“ Ultrasonic RAdar System (URAS): Arduino and Virtual Reality for a Light-Free Mapping of Indoor Environments” IEEE Sensors Journal Volume: 17 , Issue: 14 , July 15, 152017

[4] Kiruthikamani.G, Saranya.B, Pandiyan.P-“Intelligent Driver Monitoring and Vehicle Control System” IJSRD - International Journal for Scientific Research \& Development| Vol. 5, Issue 09, 2017

[5] Mohanad Mahdi Abdulkareem,Qusay Adil Mohammed ,Muhanned Mahmood Shakir-“A Short Range Radar System"Rangefinder”,

[6] Harshad D. Lajurkar, Rushikesh D. Malokar, Akash N. Karmore“ Currency Recognition Blind Walking Stick” IJIRST -International 
Journal for Innovative Research in Science \& Technology,Volume 4 ,Issue 7 ,December 2017

[7] Srijan Dubey,Supragya Tiwari, Simit Roy -“ IMPLEMENTATION OF RADAR USING ULTRASONIC SENSOR” Indian J.Sci.Res. 2017

[8] Anuj Dutt (Author), 2014, Arduino based RADAR System, Munich, GRIN Verlag.

[9] T H Nasution, E C Siagian, K Tanjung, Soeharwinto-"Design of river height and speed monitoring system by using Arduino" 10th International Conference Numerical Analysis in Engineering 2018

[10] Syed M Taha Saquib, Sarmad Hameed, Syed M Usman Ali, Raza Jafri, Imran Amin-"Wireless Control of Miniaturized Mobile Vehicle for Indoor Surveillance" ICSICCST 2013 IOP Conf. Series: Materials Science and Engineering

[11] Sami ur Rahman, Sana Ullah and Sehat Ullah-" Obstacle Detection in Indoor Environment for Visually Impaired Using Mobile Camera"CCISP 7017, IOP Conf. Series: Journal of Physics: Conf. Series 2018. 


\section{AUTHORS}

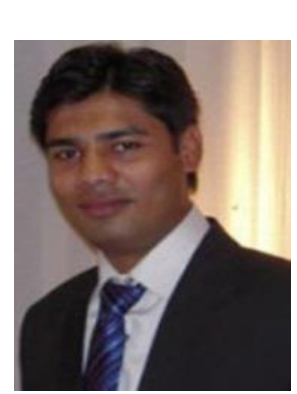

\section{Sarmad Hameed}

Sarmad Hameed is serving as Lecturer in Mechatronic Engineering Department at SZABIST. He has done his master degree in Industrial Controls and Automation Program from Hamdard University. He has earned his bachelor degree in Electronics from Sir Syed University of Engineering and Technology in 2010. His areas of interest are digital circuit designing, programming; troubleshooting, and industrial control systems design. His job description includes integration of renewable energy technologies into the electric power system.

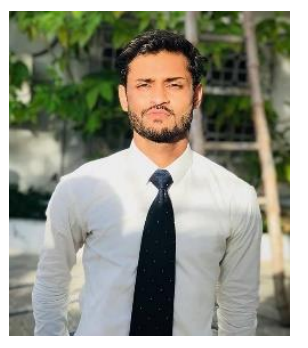

\section{Naqi Jafri}

Naqi Jafri currently enrolled in final year of B.E Mechatronics at SZABIST. His area of interest are IOT based system, robotics and embedded systems.

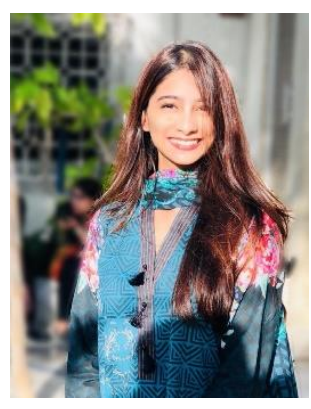

\section{Dania Rashid}

Dania Rashid is a final year student of Mechatronics Engineering department doing from SZABIST. Her area of interest are robot navigation, IOT based system and Simultaneous Localization and Mapping (SLAM).

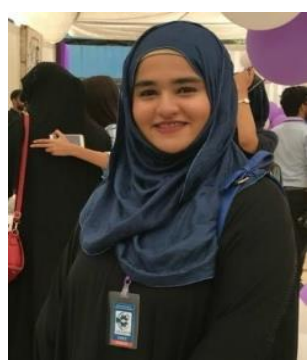

\section{Fabiha Shoaib}

Fabiha Shoaib currently enrolled in final year of B.E Mechatronics at SZABIST. Her field of interests are Autonomous \& Connected. 


\title{
DESIGN AND SIMULATION OF MEMS SHUNT CAPACITIVE SWITCH FOR LOWER SWITCHING TIME
}

\author{
Kurmendra \\ Department of Electronics \& Communication Engineering, \\ Rajiv Gandhi University, Itanagar, (India) \\ E-mail: kurmendra.nits@gmail.com
}

Rajesh Kumar

Department of Electronics \& Communication Engineering,

North Eastern Regional Institute of Science \& Technology, Itanagar, (India)

E-mail: itsrk2006@gmail.com 


\section{ABSTRACT}

As Demand of High speed devices increasing for RF and satellite communications with better accuracy, MEMS technology is considerd to be emerging technology to fulfill that need. In this paper, A MEMS shunt capacitive switch with fixed- fixed beam have been designed and simulated for numerous parameters. The parameters for study are Selection of beam material for the switch, air gap distance between electrodes and importantly the actuation voltage. For studying the effect of air gap and beam width on switching time, the air gap was varied from $0.6 \mu \mathrm{m}$ to $2.0 \mu \mathrm{m}$ and beam width from $1 \mu \mathrm{m}$ to $50 \mu \mathrm{m}$. For an actuatiuon voltage of $10.5 \mathrm{~V}$ and air gap distance of $0.6 \mu \mathrm{m}$, switching time result is $0.2 \mathrm{~ns}$ for spring constant equal to gold material.Study also considers the effect of increasing beam dimension in terms of width for a constant gap height. This syudy will be helpful for designing a MEMS capactitive switch for higher speed and for selection of proper dimension to get better performance.

\section{KEYWORDS}

Beam, Electrode, MEMS, RF, Switch, Switching time.

\section{BACKGROUND}

RF MEMS switches are considered to be a potential switching device for high speed switching applications. These types of switches have capability of replacing traditional MOSFET switch, Tunnel Switch and Pin-diode based Switch [1-4]. Many actuation mechanisms such as optical actuation, electrostatic actuation, thermal actuation and actuation by force are used for MEMS switch devices [5]. Mostly used actuation mechanism is Electrostatic actuation since it has many practical advantages such as low power requirement and small sized device. Despite of having various advantages, MEMS switches lack in switching speed and requirement of low pull in voltages [6-7]. Various techniques are currently being used to overcome the problems associated with these switches. Low spring constant materials are considered to be prominent source of material for low pull in voltage but at the same time, the switch suffers with low speed and reliability of device [8-9]. The switching speed can be increased by using a switch beam with lower mass. The switches made up of low mass materials relatively posses high speed compare to higher spring constant materials [10]. A. Kundu et al. (2010) have designed a new switch where the top metal and central conductor both are movable and considerable improvement was found in actuation voltage requirement and switching time as $20 \%$ down [11]. S. Sekhar et al. (2011) designed electrostatically actuated MEMS switch and experimental as well as FEM analysis were done. Their observation concludes that pull up time is more than pull in time which is somewhat counter part because in case of pull in there is much larger electrostatic force compare to pull up restoring force at release time [12]. C. Siegel et al, have provided the switching analysis of MEMS cantilever-based switch and article reports that measured switching time in the air medium for the switch is below $75 \mu \mathrm{s}$ which is also dependent of actuation voltage. Semiconductor effect in actuation path is responsible for $60 \mu$ s and switching time also depends on bending of the membrane [13]. Markus et al 
(2014) have reported that they have designed a MEMS switch which requires very low pull in voltage as $5 \mathrm{~V}$ and switching time is less than $10 \mu \mathrm{s}$ at $5 \mathrm{Ghz}$. The designed switch is very much usable for RF applications [14]. K. Guha et al. (2015), have proposed meander-based switch made up of Aluminum membrane and the study was done for providing very less switching time as 3 $\mu$ s for actuation voltage of $5 \mathrm{~V}$ [15]. K. Guha et al. (2018) have also proposed a new analytical model for analysis of switching time for perforated MEMS switch. They have presented modified Mejis and Fokkema's model and the model is capable of evaluating switching time for uniform as well as nonuniform meander-based switch designs. Results were plotted between switching time and displacement for uniform and non-uniform meander designs with different Vs and materials [16].

In this article, a Shunt capacitive MEMS switch is designed which is capable of working at very high frequency range $20-100 \mathrm{Ghz}$ approximately.. The MEMS switches have also been designed by other researchers having different kind of structures. But these designed switches have many disadvantages such as insertion loss, isolation loss, return loss and very important switching time.The previously published articles in the domain have addressed about losses associated with their designed switches but only a few articles have presented switching time analysis [refer table.2 ]. Firstly, the structure presented in this paper was optimized in terms of different size dimensions as well as the selection of materials involved a rigorous literature review and were so chosen that the disadvantages associated with previously designed switches could be overcome [17-18]. After designing the switch, we have done many static analysis such as pull in voltage analysis and RF analysis which have already been published in a journal of repute [21]. We found that the designed switch is showing a great improvement in terms of insertion, isolation and return loss parameters [21] Here, An analysis of the MEMS switch considering switching time is presented in terms of dielectric constant, voltages, air gap and width of the beam material.

In the proposed work we have taken most promising issue related to the MEMS switch that is switching time. Our work has shown tremendous improvement in terms of switching time as well as losses associated with. The switch designed on MEMS technology generally have one disadvantages of having larger switching time which has been significantly improved and which is presented in the paper.

\section{DESIGN OF PROPOSED MEMS SHUNT SWITCH AND WORKING}

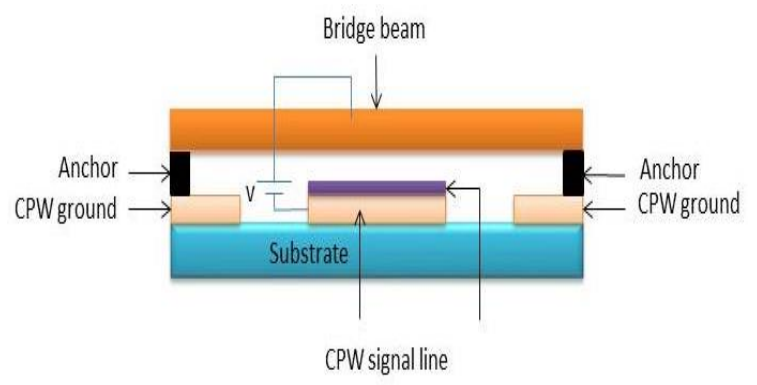

Figure 1. Schematic of proposed RF MEMS shunt switch. 
The schematic of designed MEMS shunt switch is shown in the Figure1. The switch consists of silicon substrate, dielectric layer (Si3N4) placed over CPW transmission line and gold material is used for beam bridge, beam supporting anchors and transmission line central conductor as well as CPW ground. There is an air gap g0 between Au top electrode and dielectric layer above CPW signal line. The switch is actuated by using very common actuation technique that is electrostatic actuation. Initially there will not be applied any actuation voltage and the signals in the signal line go without any interruption. When a voltage is supplied between beam and down electrode (CPW signal line) as shown in fig. 1, an electrostatic force is developed on switch membrane (beam) and this force pulls down the beam. When this beam touches dielectric material, it forms a path between signal line and beam to the CPW ground for signals coming from CPW signal line, thus signal goes to CPW ground, the switch is called to be in OFF state. In the actuation and switching analysis, the supply voltage, air gap and material properties play very important role.

\section{FORMULATION \& SWITCHING PERFORMANCE ANALYSIS}

RF MEMS switch generally offers a better performance characteristic when considered for low insertion loss, high isolation and low return loss. The performance characteristics of the switch does not only depend on these losses but also depends on the speed to make open and close circuit, generally termed as switching speed. Switching speed can be improved by employing different techniques such as TMPS technique [11] where the electrode as well as signal conductor move to offer a better switching speed. In this section, we are going to include some important switching equations for switching time analysis.

The switching time in terms of applied voltage $(V s)$, pull in voltage $(V p)$ and operating frequency can be given as [19]

$t s=3.67 \frac{V p}{V s \omega 0}$

(1)

Where, 'ts' is the switching time of the designed switch, $V p$ is the pull in voltage $(10.5 \mathrm{~V}), \mathrm{Vs}$ is the supplied voltage $(V s=1.4 \mathrm{Vp})$ and $\omega 0$ is the operating frequency or resonance frequency of the switch. Spring constant of the material specifically used for switch membrane is important parameter for the switching time analysis of the switch. An equation for switching time in terms of spring constant of beam membrane can be obtained by putting $V s=1.4 \mathrm{Vp}$ and $\omega 0=\left(\frac{k}{m e}\right)^{1 / 2}[16]$

ts $=2.62\left(\frac{m e}{k}\right)^{1 / 2}$

Where, ' $m e$ ' is the effective mass and ' $k$ ' is the spring constant of the beam membrane for the designed switch.

The switching time in terms of gap height g0 and width of the membrane can be approximated and give as [11]

$t s=\frac{1}{V s}\left(\frac{4 m e * g 0^{3}}{\varepsilon 0 * W}\right)^{1 / 2}$ 
Where, $g 0$ is the initial airgap between the membrane and signal line, $\varepsilon 0$ is the free space permittivity and $W$ is the width of the switch beam membrane.

The equations which are provided above helps in analyzing the switch performance in terms supplied voltage, spring constant, air gap heights and width of the top metal beam.

\section{RESULT \& DISCUSSIONS}

A MEMS capacitive shunt switch was designed as shown in the Figure 1. The design parameters and materials used in the proposed switch is given in the Table.1. The operating frequency of the switch was calculated using coplanar waveguide calculator [20] and was verified using COMSOL Multiphysics 5.1 software.

Table 1. Design parameters and materials used of the proposed switch.

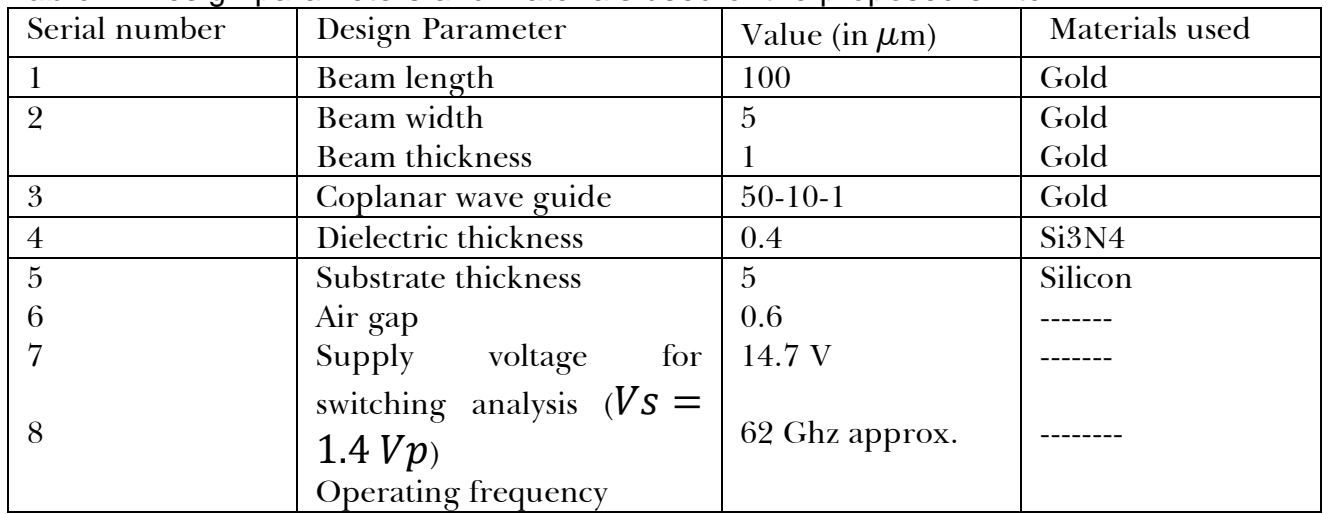

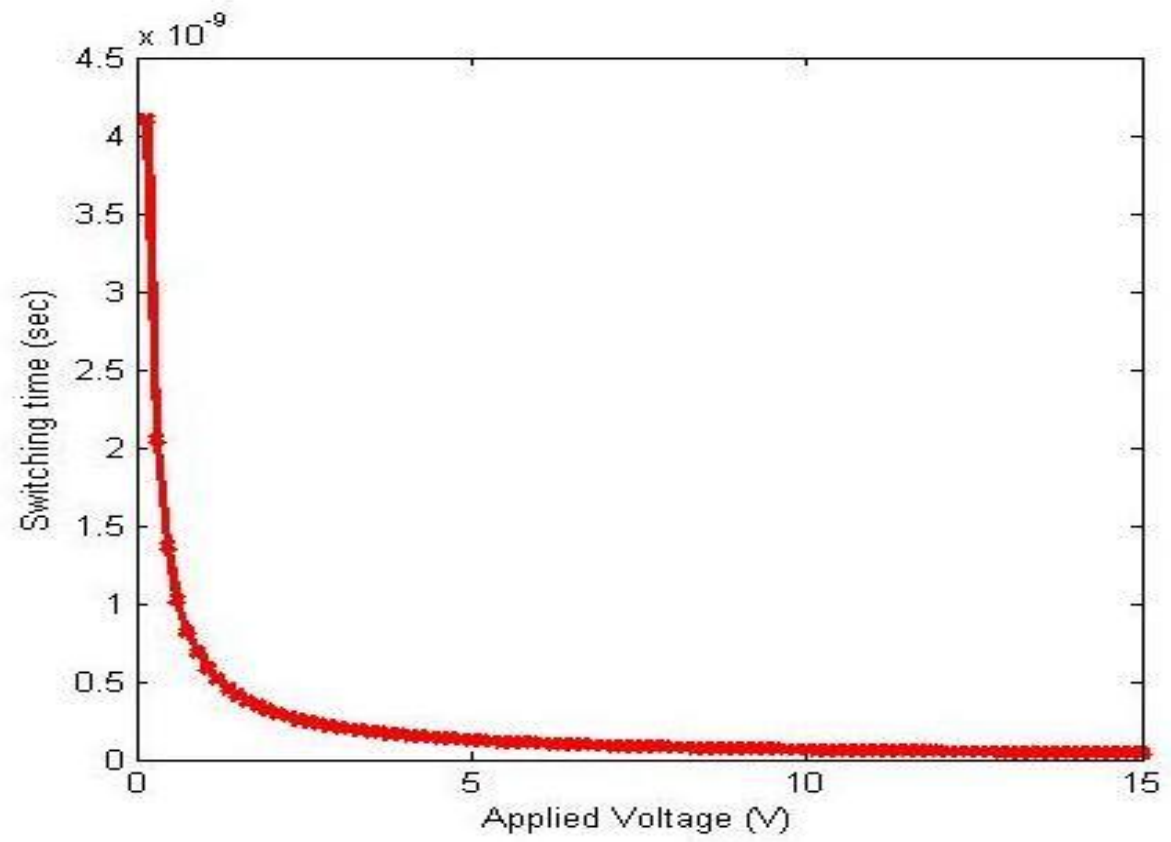

Figure 2. Switching time variation in terms of applied voltage $(\mathrm{Vs}=1.4 \mathrm{Vp})$.

In the Figure 2, result for switching time for the sweep of $0-15 \mathrm{~V}$ in supply voltage is given. From the plot we can understand that in the voltage range 0 $5 \mathrm{~V}$, switching time is more compare to switching time for $5-15 \mathrm{~V}$. our designed 
switch posses pull in voltage about $10.5 \mathrm{~V}$ for which we obtain the switching time as low as $0.2 \mathrm{~ns}$.

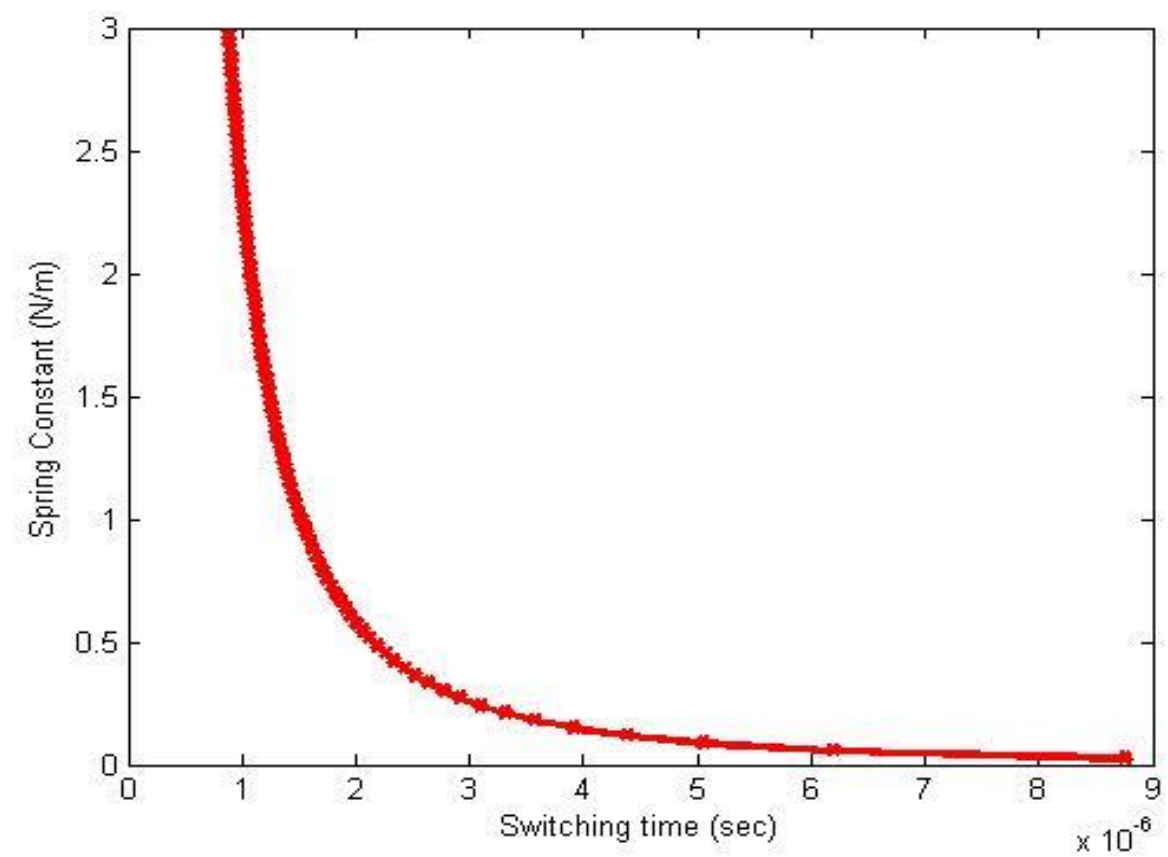

Figure 3. Switching time variation for varying spring constant of beam material.

Figure 3 illustrates, the curve between spring constant for the beam material and switching time. It can be clearly concluded that the switch made up of high spring constant value is going to require very less switching time compare to beam materials with low spring constant thus enables switch to work at high speed.

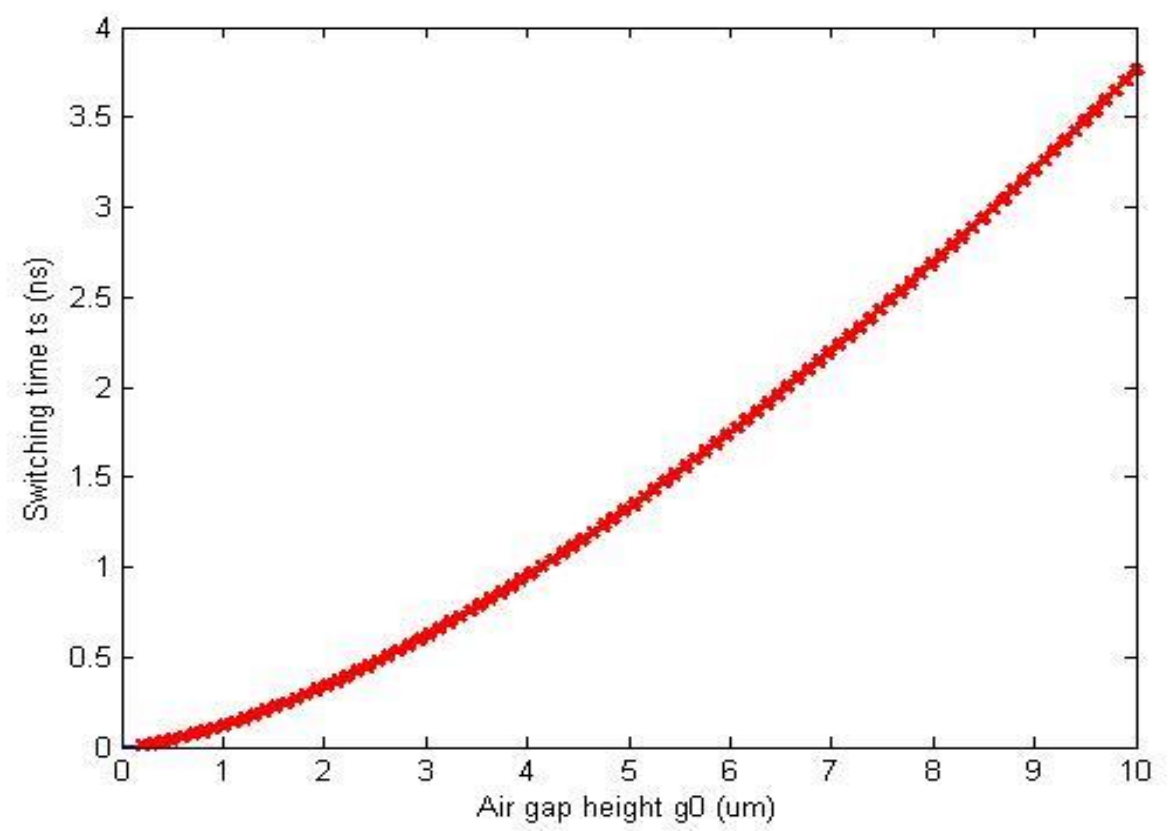

Figure 4. Switching time as function of varying air gap height between beam electrode and dielectric layer above signal line. 
Figure 4 depicts the result between the air gap heights and switching time for designed switch, as per the literature the lower the gap between top and bottom electrode demands lower switching time but lowering of this gap is permissible only up to certain limit since there should be enough gap to apply supply voltage $(V s=1.4 \mathrm{Vp})$. In Figure 4 it can be observed that as we are approaching towards higher air gap, the requirement of switching time is also increasing which considerably reduces the speed of the switch.

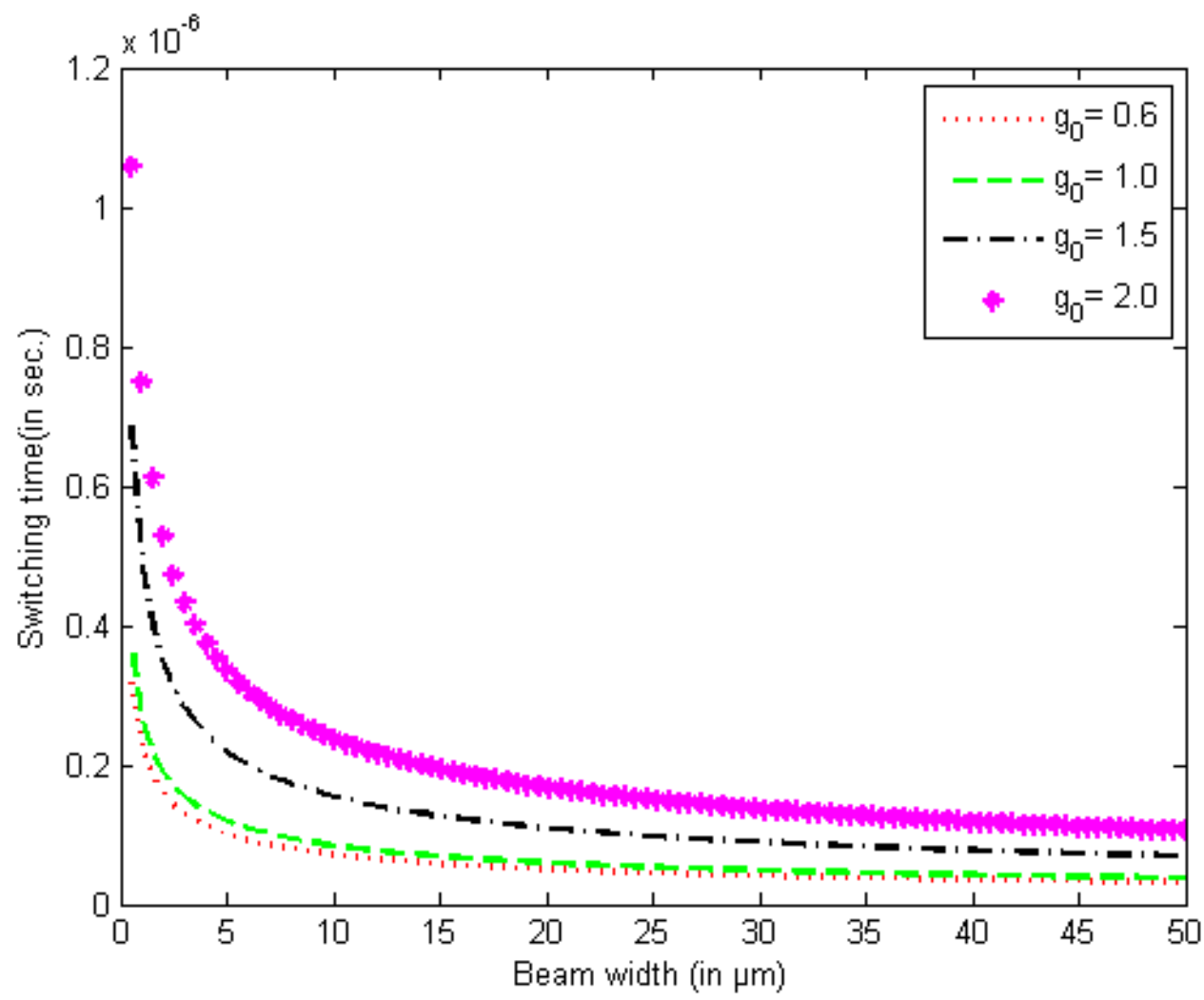

Figure 5. Switching time variation for different beam width for air gap go,g1,g2 and g3 .

As per the equation (3) of the section.3, we can see that the beam width does play an important role for switching speed or switching time of the device. In the Figure 5, the result for varying width of the beam and switching time is plotted for constant air gap $(\mathrm{g} 0=0.6 \mathrm{um}, \mathrm{g} 1=1.0 \mathrm{um}, \mathrm{g} 2=1.5 \mathrm{um} \& \mathrm{~g} 3=$ $2 \mathrm{um}$ ). Arrow sign in the Figure 5 is kept for showing increasing air gap height direction. as we keep on increasing the width of fixed- fixed beam of the switch, the switching time required is getting lesser. While choosing greater beam width, we should note that for a miniaturized device, there is need of using beam with lesser beam width. The effect of increasing air gap between beam electrode and lower electrode are considerably high for the same sweep of beam width. In the Figure 5, it can be observed that as we are increasing this gap the switching time requirement is going to be very high compare to lesser beam width. Thus, there is a need for trade off between the switch parameters such as air gap, beam width, applied voltage and selection of materials for improving the switching time which ultimately improves the switching speed. 
Table 2. Compared results obtained with various losses, Frequency of operation and switching time.

\begin{tabular}{|l|l|l|l|l|l|l|}
\hline Sr. No. & $\begin{array}{l}\text { Insertion } \\
\text { loss }\end{array}$ & Isolation & $\begin{array}{l}\text { Return } \\
\text { loss }\end{array}$ & $\begin{array}{l}\text { Switching } \\
\text { time }\end{array}$ & $\begin{array}{l}\text { Frequency } \\
\text { of } \\
\text { operation }\end{array}$ & References \\
\hline 1 & $0.29 \mathrm{~dB}$ & $20.5 \mathrm{~dB}$ & ------ & Not done & $35 \mathrm{Ghz}$ & $\begin{array}{l}\text { Muhua li et al } \\
{[2017][22]}\end{array}$ \\
\hline 2 & $-0.4 \mathrm{~dB}$ & $80 \mathrm{~dB}$ & ------ & Not done & $20 \mathrm{Ghz}$ & $\begin{array}{l}\text { Guha et al. } \\
{[2016][23]}\end{array}$ \\
\hline 3 & $-0.44 \mathrm{~dB}$ & $-20 \mathrm{~dB}$ & $-16 \mathrm{~dB}$ & Not done & $\begin{array}{l}0.6-40 \\
\mathrm{Ghz}\end{array}$ & $\begin{array}{l}\text { T. Laxmi } \\
\text { Narayana et } \\
\text { al [2017] [24] }\end{array}$ \\
\hline 4 & ------ & ------- & -------- & $<10 \mathrm{us}$ & $5 \mathrm{Ghz}$ & $\begin{array}{l}\text { M. Gatzsch et } \\
\text { al [2014] [14] }\end{array}$ \\
\hline 5 & $-0.05 \mathrm{~dB}$ & $-12 \mathrm{~dB}$ & $-45 \mathrm{~dB}$ & $0.2 \mathrm{~ns}$ & $61.5 \mathrm{Ghz}$ & \begin{tabular}{l} 
Our work \\
\hline
\end{tabular} \\
\hline
\end{tabular}

A comparision have been shown in Table 2 between our designed MEMS shunt capacitive switch and switch reported in other articles. Through comparision it was found that our designed switch results in a much improved performance in terms of losses and switching speed.

\section{CONCLUSION}

The paper presents the performance analysis of a new and simple shunt capacitive switch design in terms of switching time. The analytical equations were presented for the analysis. Specifically switching time was analytically analyzed on four parameters such as supplied voltage, spring constant of material, initial air gap heights and width of the material. From the analysis of the results, it can be concluded that trade off between supply voltage and switching time is required since high switching is achieved for high supplied voltage but a device with high supply voltage is not recommended. High spring constant materials results in less switching time which practically improves switching speed. A switch with larger beam width and lesser air gap is efficient for high switching speed. This study will surely be useful for researchers working in the area of MEMS switch design for high frequency applications.

\section{ACKNOWLEDGEMENT}

This research work has been carried out in MEMS laboratory, Department of ECE, Rajiv Gandhi Central University, Itanagar, INDIA. 


\section{REFERENCES}

1. G.M. Rebeiz "RF MEMS Theory, Design and Technology" Wiley, New Jersey (2003)

2. A. DeHon, "Array-based architecture for FET-based, nanoscale electronics," in IEEE Transactions on Nanotechnology, vol. 2, no. 1, pp. 23-32, Mar 2003. doi: 10.1109/TNANO.2003.808508

3. F. Gentili, L. Urbani, G. Bianchi, L. Pelliccia and R. Sorrentino, "PIN diode-based 4-channel switched filter bank with low-power, TTL-compatible Driver," IEEE MTT-S International Microwave Symposium (IMS2014), Tampa, FL, 2014, pp. 1-4. doi: 10.1109/MWSYM.2014.6848379

4. A. Chaney, M. Qi, S. M. Islam, H. G. Xing and D. Jena, "GaN tunnel switch diodes," 2016 74th Annual Device Research Conference (DRC), Newark, DE, 2016, pp. 1-2. doi: 10.1109/DRC.2016.7548409

5. B. G. Sheeparamatti, Prashant Hanasi, Vanita Abbigeri, "Critical Analysis of Different Actuation Techniques for a Micro Cantilever", World Academy of Science, Engineering and Technology International Journal of Mechanical and Mechatronics Engineering Vol:9, No:11, 2015.

6. M. Solanska, M. Markovic and M. Dado, "Time analyses of SRFORP and S\&C reservation protocols in optical networks with 2D MEMS switches," ELEKTRO, Strbske Pleso, 2016, pp. 88-91. doi: 10.1109/ELEKTRO.2016.7512041

7. Mahesh Angira, Kamaljit Rangra, "A novel design for low insertion loss, multi-band RF-MEMS switch with low pull-in voltage", Engineering Science and Technology, an International Journal, Volume 19, Issue 1, 2016, Pages 171-177, ISSN 2215-0986, https://doi.org/10.1016/j.jestch.2015.07.001.

8. C.-Liang Dai, H.-Ming Hsu, M.-Chang Tsai, M.-Ming Hsieh, MingWei

Chang,

"Modeling and fabrication of a microelectromechanical microwave switch",

Microelectron. J. 38 (2007) 519-524

9. D. Hah, E. Yoon, S. Hong, "A low voltage actuated micromachined microwave

switch using torsion springs and leverage", IEEE Trans. Microwave Theory

Tech. (2000) 2540-2545

10. Bonthu, M. \& Sharma, A.K. "An investigation of dielectric material selection of RF-MEMS switches using Ashby's methodology for RF 
application”,s Microsyst Technol (2018) 24: 1803. https://doi.org/10.1007/s00542-017-3539-x

11. A. Kundu , S. Sethi , N.C. Mondal, B. Gupta, S.K. Lahiri , H. Saha, "Analysis and optimization of two movable plates RF MEMS switch forsimultaneous improvement in actuation voltage and switching time”, Microelectronics Journal 41 (2010) 257-265

12. Shekhar, S, Vinoy , KJ , Ananthasuresh, GK,"Switching and Release Time Analysis of Electrostatically Actuated Capacitive RF MEMS Switches". (2011) In: IFSA, 130 (7). pp. 77-90.

13. C. Siegel, V. Ziegler, C. von Wächter, B. Schönlinner,U. Prechtel, H. Schumacher, "Switching speed analysis of low complexity RF-MEMS switches" https://duepublico.uni-duisburg-

essen.de/servlets/DerivateServlet/Derivate-

14694/Final Papers/GM0029-F.pdf

14. Markus Gaitzsch, Steffen Kurth, Sven Voigt, Sven Has, Thomas Gessner , "Analysis of Au metal-metal contacts in a lateral actuated RF MEMS switch", International Journal of Microwave and Wireless Technologies, 2014, 6(5), 481-486

15. Koushik Guha, Prerana Roy, AnganaNath, Srimanta Baishya, "Switching Time Analysis of MEMS Shunt Switch using MATLAB", 2nd International Conference on Microelectronics, Circuits and Systems,Micro2015.

16. K. Guha, N. M. Laskar, H. J. Gogoi, K. L. Baishnab, K. Srinivasa Rao, "A new analytical model for switching time of a perforated MEMS switch", Microsyst Technol (2018). https://doi.org/10.1007/s00542018-3803-8

17. Kurmendra \& Kumar, R. , "Design analysis, modeling and simulation of novel rectangular cantilever beam for MEMS sensors and energy harvesting applications” ,Int. j. inf. tecnol. (2017) 9: 295. https://doi.org/10.1007/s41870-017-0035-6

18. Ashby, M. F., "Materials Selection in Mechanical Design", 2nd Edition, Butterworth-Heinemann, Oxford, UK, 1999.

19. A. K. Sharma and N. Gupta, "Switching time analysis for non-uniform serpentine flexure based RF-MEMS switches," Students Conference on Engineering and Systems (SCES), Allahabad, 2013, pp. 1-4. doi: 10.1109/SCES.2013.6547531

20.

Waveguide

Calculator,

$\underline{\text { http:// }}$ www.microwave101.com/encyclopedia

21. Osor Pertin, Kurmendra, "Pull-in-voltage and RF analysis of MEMS based high performance capacitive shunt switch", Microelectronics 
Journal, Volume 77, 2018, Pages 5-15, ISSN 0026-2692, https://doi.org/10.1016/j.mejo.2018.05.001.

22. Muha li, Jiaho Zhao, Zheng You, Guonghong Zhao, "Design and fabrication of a low insertion loss capacitive RF MEMS switch with novel micro-structures for actuation", Solid-State Electronics Volume 127, 32-37 (2017)

23. Koushik Guha, Mithlesh Kumar, Ajay Parmar, Srimanta Baishya, "Performance analysis of RF MEMS capacitive switch with non uniform meandering technique”, Microsyst Technol 22: 2633 (2016).

24. T. Lakshmi Narayana, K. Girija Sravani, K. Srinivasa Rao,Wei Meng " Design and analysis of CPW based shunt capacitive RF MEMS switch", Cogent Engineering, 4:1, (2017)

DOI: $\underline{10.1080 / 23311916.2017 .1363356}$ 
Design and simulation of mems shunt capacitive switch for lower switching time

DOI: http://dx.doi.org/10.17993/3ctecno.2019.specialissue.15 


\title{
AUTOMERLIN MOBILE ROBOT'S BILATERAL TELECONTROL WITH RANDOM DELAY
}

\author{
Aamir Shahzad \\ Department of Mechanical Engineering, The University of Lahore, Lahore, \\ (Pakistan) \\ E-mail: aamir.shahzad1@me.uol.edu.pk \\ Muhammad Salahudin \\ Department of Mechanical Engineering, The University of Lahore, Lahore, \\ (Pakistan) \\ E-mail: muhammadsalahuddin@hotmail.com \\ Iqbal Hussain \\ Department of Mechanical Engineering, The University of Lahore, Lahore, \\ (Pakistan) \\ E-mail:iqbal.hussain@me.uol.edu.pk
}




\section{ABSTRACT}

The main focus of this work is to design a bilateral telecontrol of a mobile robot AutoMerlin through the Internet. The Internet has an inherent delay, packet drop, out of order data transmission, duplication, and other impediments as a communication channel. These factors cause the system to become unstable and difficult to control through the Internet. The velocity tracking becomes really hard and the force feedback also rises to an unacceptable level due to delay and other impediments. In order to address these issues, a power based TDPC (Time Domain Passivity Control) has been utilized in this work for the development of stable telecontrol. This approach is based on energy. The energy has been classified as positive and negative energy to make passivity analysis independent of monitoring of net system energy in real time. Thus, monitoring the net energy output at each port enables the extension of TDPC for delayed systems called TDPN (Time Delay Power Network). TDPN helps in velocity/force tracking. It transmits velocity/force unaltered by rejecting the active energy. PO (Passivity Observers) indicate the active behavior and the PC (Passivity Controllers) dissipate extra surplus energy to keep the system stable and passive all times. The performance has been tested and plotted to show the effectiveness of the bilateral controller under random delay and other limitations.

\section{KEYWORDS}

Telecontrol; Haptic force; Joystick; Unstructured environment; Slave robot; Time delay power network; Random delay.

\section{INTRODUCTION}

Bilateral telecontrol of a mobile robot can be defined as control of the robot from a remote location while receiving force feedback from it as shown in Figure 1. The complete configuration of telecontrol comprises of a human operator, a haptic device connected to a computer having client algorithm, communication medium, slave robot equipped with server algorithm and remote environment [1-4]. The human operator applies the required maneuvers to the master haptic device which translates it into inputs for the slave robot in the remote environment. The desired inputs of the master device travel through some communication medium to the slave robot. These commands/instructions are executed by the slave robot in order to manipulate the remote environment. The effect of the environment is a reactive force on the slave robot. This force is sent as force feedback from a remote location to master haptic device. This force feedback is played over the haptic joystick. The human operator experiences the force feedback via haptic device and gets a sensation of actually manipulating the remote location directly. Telecontrol is a combination of different subsystems that exchange energy. The energy is exchanged by forwarding velocity and receiving force feedback [5-7]. Passivity control is based on system's net energy and it is an efficient tool for the stability analysis of the bilateral telecontrol. The combination of passive subsystems is always a passive system [8]. Hence, to ensure the passivity of the system, the subsystems can be analyzed to be passive all times. The stability based on the 
mathematical model of the system imposes conservative and strict rules on the performance of the system. It cannot easily tolerate the delay in the system and also requires accurate information about the remote environment [9-10]. The passivity is independent of a mathematical model of the system and is based on energy balance.

The master and slave are passive as they dissipate energy but when they are connected by means of any communication medium, then that medium can behave actively by inducing surplus energy in the system. The time delay is the main source of instability and activity too. A passivity-based approach using wave variables has been proposed by Niemeyer [11]. Wave variables have been utilized to develop the teleoperation with force reflection. Similarly, the scattering approach has been presented by Anderson and Spong [12]. These approaches have guaranteed the passive telecontrol at the cost of over dissipation of energy. This over dissipation of energy resulted in conservative performance [13-15]. To cope with these issues a remarkable approach based on TDPC has been presented by Ryu and Hannaford [16]. TDPC has two main elements called PO (Passivity Observer) and PC (Passivity Controller). PO keeps the track of energy entering and leaving the system to estimate net energy. The PG takes all the needed measures to dissipate surplus energy introduced in the control loop by various means. TDPC is based on calculation of net energy in real time to perform necessary control action. But in case of time delay due to distance or communication through a shared medium, the observation of net energy is not possible in real time. Hence the controller cannot take any action against the active energy. Therefore, Artigas has presented an extension to TDPC for the delayed system [17]. By using an Electrical/ Mechanical analogy, it has been proven that instability occurs due to non-

block
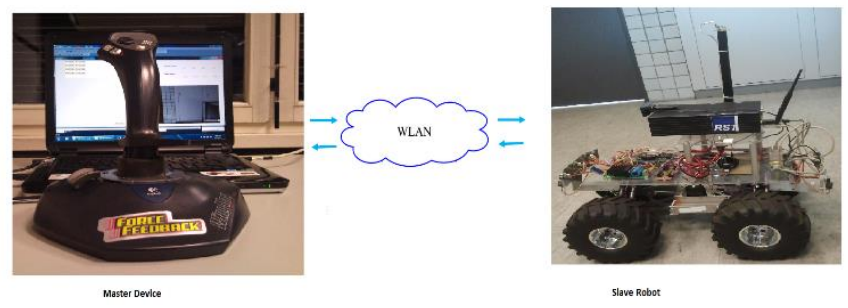

passive communication during

Figure 1. The bilateral telecontrol of AutoMerlin.

bilateral telecontrol with constant or random delay. A communication block has been modelled as a lossless entity which acts as a passive element even with time delay [18]. Teleoperator's stability has been guaranteed without limiting the bandwidth. This control approach ensures passivity of all subsystems in the closed-loop i.e. master, communication block and slave. Once the passivity is attained, the system is stable even though there are limitations and disturbances. These limitations are human operator dynamics, variable and unknown communication delay. Force feedback has been modelled as summation of two forces i.e. the virtual force and friction. The virtual force is based obstacles in the vicinity of the robot in a remote environment and friction 
is between ground and wheels. The virtual force calculated due to obstacles is a function of distance and velocity travelled by mobile robot. The complete method and its elaboration have been given in [4]. This force acts on the robot while it is moving in a remote environment is played over the haptic device as force feedback to the human operator. The velocity, force telecontrol is shown in Fig. 2. The slave robot is receiving the velocity command from the master device and delayed environmental force from the slave is reflected back.

Section I of this paper is Introduction about telecontrol, different approaches used in teleoperation and their pros and cons. Problem description has been presented in Section II with different plots to clearly illustrate the issues. Section III briefly describes the TDPN. It has mathematical modelling for the passivity of the network with random delay. The energy relations have been explained in it. Section IV has experimental results to show the performance of the teleoperation with TDPN. Section V has Conclusion and Future Work.

\section{PROBLEM DESCRIPTION}

The objective of telecontrol is to establish a close coordination among the interacting subsystems i.e. the haptic device and slave robot along with some limitations like limited bandwidth and random or constant time delay. Therefore, to elaborate the problem, there are some plots which have been included in this section to realize the actual issues. The blue line in the Figure 3 , shows the master haptic device's velocity and the green line represents the velocity of the slave robot. It is vivid that the slave velocity is more than the desired velocity set by the master device. This is due to the activity of the Internet because the master velocity travels through it and it has the delay in it and also other limitations like duplication, drop in packets and change in the order of data etc. [4]. The blue line indicates energy input at the master side while the green line is showing energy output and net energy is represented by a red line in Figure 4.

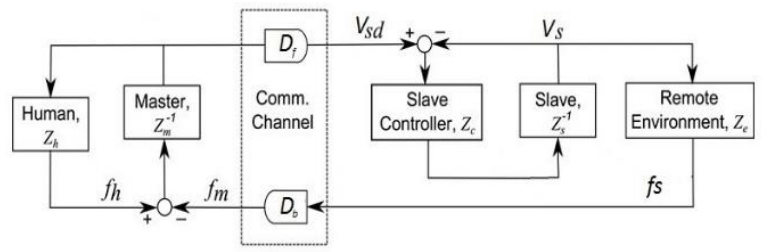

Figure 2. Block diagram of telecontrol.

It is clear from the plot that the net energy is negative and it is accumulating because active energy is not being dissipated. Figure 5, shows the two forces i.e. virtual force on the slave robot and force feedback on master haptic device. During certain intervals like after 4 seconds, the force feedback is larger as compared to the environmental force acting on the slave. Due to surge in force, the energy output is greater as compared to the energy input. This implies that the shared medium i.e. Internet is adding energy into the system to make it active and unstable. The energy comparison between net energy, input energy and output energy from slave to master has been plotted in the Figure 6. 


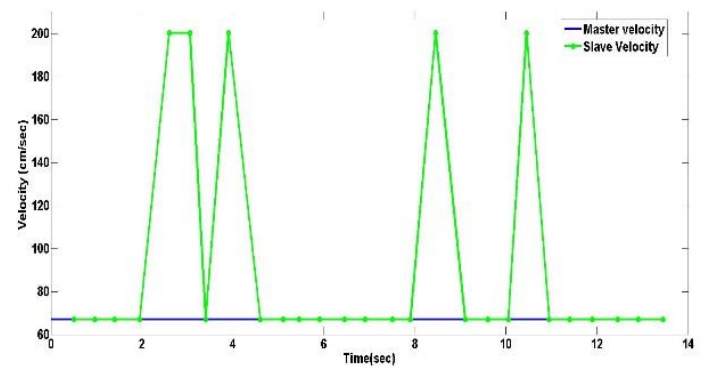

Figure 3. Linear velocities of both robots.

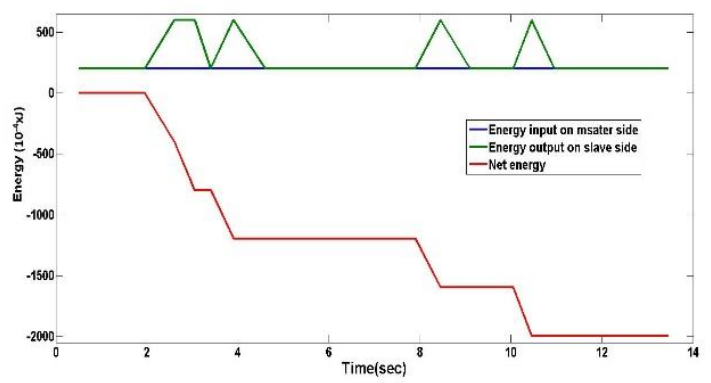

Figure 4. Energy flow master to slave.

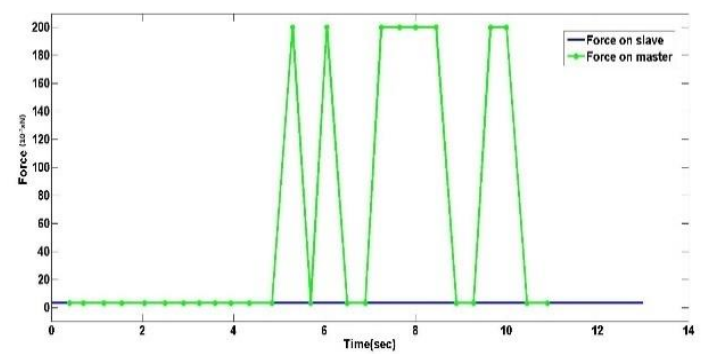

Figure 5. Force comparison on both robots.

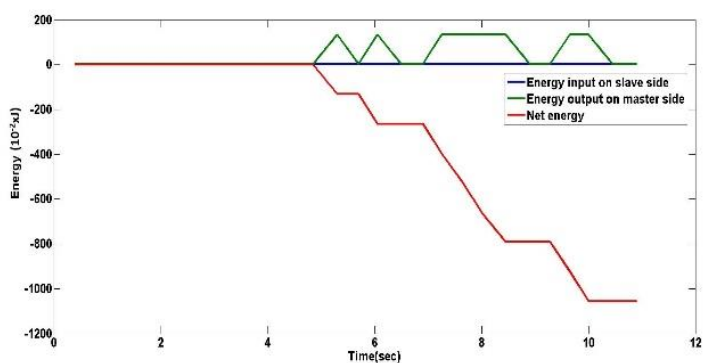

Figure 6. Energy flow slave to master.

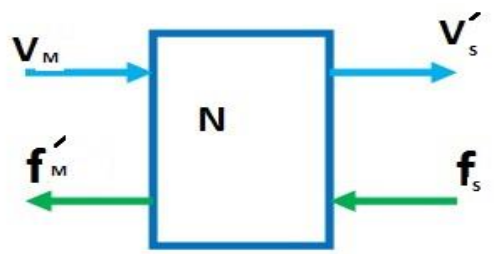

Figure 7. A Two-port network as TDPN.

\section{TDPN (TIME DELAY POWER NETWORK)}

The communication medium is adding active energy in the passive telecontrol system while reciprocating the velocity/force. Therefore, in order to solve the issue of active energy, the communication medium has been modelled as a Two-port network in which velocity/force move contrary to each other as depicted in Fig. 7. Each port corresponds to a real-time velocity/force signal 
and its conjugate delayed signal. The velocity force multiplication is power and the accumulation of this power over time results in energy. This modification of the communication medium is called the TDPN. TDPN acts in the same manner as the conventional communication medium but it assists in resolving the issue of random time delay inherent to a network. Instead of exchange of velocity/force, a non-conventional concept is used i.e. transfer of energy at each port. Positive and negative energies are segregated at each port. Positive energy is getting in while negative energy is getting out at each port. The analysis of energy is done with respect to input energy at the output of each port. PO calculates the net energy output at each port. Whenever PO finds out an active energy presence, then the relevant PC takes the corrective measures to dump extra active energy to make the system passive again. This leads to a stable telecontrol as shown in Fig. 8. This approach separates the energy flow in the backward and forward direction and there is no connection between these two quantities for passivity analysis of the whole system.

\section{A. Passivity of TDPN}

Figure 7 shows the velocities and forces entering and leaving a Two-port network. Power is the product of these two variables. The total power of the network can be written as given in (1).

$$
P_{N}(t)=P_{M}(t)+P_{S}(t)
$$

$P_{M}(t)(\mathrm{M}=$ master $)$ is the power on the master side of a Two-port network and

$P_{S}(t)$ (S=slave) is the power on the slave side while $P_{N}(t)(\mathrm{N}=$ network $)$ is the power of the network. The energy at both sides of the network is described as

$$
\begin{aligned}
& E_{M}(t)=\int_{0}^{t} P_{M}(\tau) d \tau \\
& E_{S}(t)=\int_{0}^{t} P_{S}(\tau) d \tau \\
& E_{M}(t)=\int_{0}^{t} f_{M}^{\prime}(\tau) V_{M}(\tau) d \tau \\
& E_{S}(t)=\int_{0}^{t} f_{S}(\tau) V_{S}^{\prime}(\tau) d \tau
\end{aligned}
$$

$E_{M}(t), E_{S}(t)$ are the energies on master side and slave side respectively. Hence, to keep the network passive, the following condition should prevail.

$$
P_{N}(t) \geq 0, \quad \forall t \geq 0
$$

$E_{M}(t), E_{S}(t)$ are not available due to time delay simultaneously. Therefore, in order to solve this issue the positive and negative power has been taken at each port so that net energy can be calculated in the presence of delay. 
$P_{M}^{+}(t)=P_{M}(t) \quad \forall t \geq 0 \&$

$f_{M}^{\prime}(t) V_{M}(t) \geq 0$

$P_{M}^{-}(t)=-P_{M}(t) \quad \forall t \geq 0 \&$

$f_{M}^{\prime}(t) V_{M}(t) \leq 0$

$P_{S}^{+}(t)=P_{S}(t) \quad \forall t \geq 0 \&$

$f_{S}(t) V_{S}^{\prime}(t) \geq 0$

$P_{S}^{-}(t)=-P_{S}(t) \quad \forall t \geq 0 \&$

$f_{S}(t) V_{S}^{\prime}(t) \leq 0$

Both robots positive and negative energies are

$$
\begin{array}{ll}
E_{M}^{+}(t)=\int_{0}^{t} P_{M}^{+}(\tau) d \tau & \forall t \geq 0 \\
E_{M}^{-}(t)=\int_{0}^{t} P_{M}^{-}(\tau) d \tau & \forall t \geq 0 \\
E_{S}^{+}(t)=\int_{0}^{t} P_{S}^{+}(\tau) d \tau & \forall t \geq 0 \\
E_{S}^{-}(t)=\int_{0}^{t} P_{S}^{-}(\tau) d \tau & \forall t \geq 0
\end{array}
$$

$E^{\text {in }}(t)$ is positive and $E^{\text {out }}(t)$ is negative entering and leaving the port respectively at each side.

$$
\begin{array}{ll}
E_{M}^{\text {in }}(t)=E_{M}^{+}(t) & \forall t \geq 0 \\
E_{M}^{\text {out }}(t)=E_{M}^{-}(t) & \forall t \geq 0 \\
E_{S}^{\text {in }}(t)=E_{s}^{+}(t) & \forall t \geq 0 \\
E_{S}^{\text {out }}(t)=E_{M}^{-}(t) & \forall t \geq 0 \\
E_{N}(t)=E_{M}(t)+E_{S}(t)
\end{array}
$$

Overall net energy of whole system is given in (20).

$$
\begin{aligned}
& E_{N}(t)=E_{M}^{\text {in }}(t)-E_{M}^{\text {out }}(t)+ \\
& E_{S}^{\text {in }}(t)-E_{S}^{\text {out }}(t)
\end{aligned}
$$


$E_{M \rightarrow S}(t)$ is the energy from master haptic device to slave robot and $E_{S \rightarrow M}(t)$ from slave robot to haptic device.

$$
\begin{aligned}
& E_{N}(t)=E_{M \rightarrow S}(t)+E_{S \rightarrow M}(t) \\
& E_{M \rightarrow S}(t)=E_{M}^{\text {in }}(t)-E_{S}^{\text {out }}(t) \\
& E_{S \rightarrow M}(t)=E_{S}^{\text {in }}(t)-E_{M}^{\text {out }}(t)
\end{aligned}
$$

The network is passive until the (24) and (25) inequalities are satisfied.

$$
\begin{aligned}
& E_{M \rightarrow S}(t) \geq 0 \\
& E_{S \rightarrow M}(t) \geq 0
\end{aligned}
$$

Df is the delay time from master to slave called forward delay and $\mathrm{Db}$ is the backward delay from slave robot to haptic device. The net energies with a forward delay Df and a backward delay Db are given in (26) and (27).

$$
\begin{aligned}
& E_{M \rightarrow S}(t)=E_{M}^{\text {in }}\left(t-D_{f}\right)-E_{S}^{\text {out }}(t) \\
& E_{S \rightarrow M}(t)=E_{S}^{\text {in }}\left(t-D_{b}\right)-E_{M}^{\text {out }}(t)
\end{aligned}
$$

\section{B. Passivity observer}

$E_{M}^{O b s}(n), E_{S}^{O b s}(n)$ are observers at each side as given in (28) and (29). $\mathrm{n}$ represents the random time interval between two sample time. $\alpha_{M}$ in (28) is master controller and $\alpha_{S}$ in (29) is slave controller.

$$
\begin{aligned}
& E_{M}^{o b s}(n)=E_{M}^{o b s}(n-1)+\left(E_{M}^{i n}\left(n-D_{f}\right)-\right. \\
& \left.E_{S}^{o u t}(n)+\alpha_{M}(n-1) V_{M}(n-1)^{2}\right) \\
& E_{S}^{o b s}(n)=E_{S}^{o b s}(n-1)+\left(E_{S}^{\text {in }}\left(n-D_{b}\right)-\right. \\
& \left.E_{M}^{o u t}(n)+\alpha_{S}(n-1) f_{S}(n-1)^{2}\right)
\end{aligned}
$$

\section{Passivity Controller}

To realize a stable telecontrol, it is necessary to dump active energy which is introduced by the communication medium into the telecontrol system. 


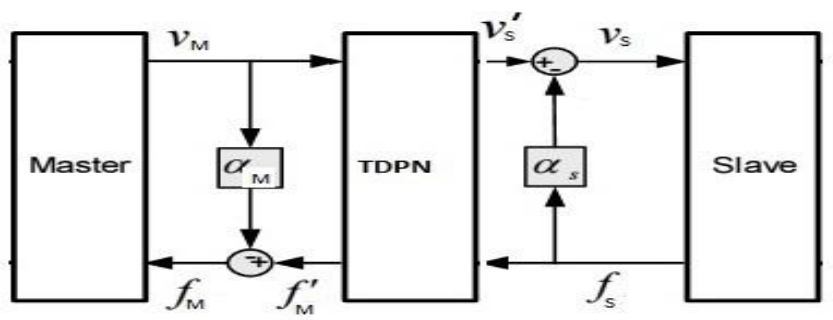

Figure 8. Master and slave controllers.

Designed $\alpha_{M}$ and $\alpha_{S}$ behave as dissipative elements on master and slave side of the TDPN respectively. The dissipation action reduces the effect of surplus energy to minimal. $\alpha_{M}, \alpha_{s}$ provide necessary controller action when the value of the observed energy is negative. The corrective measures are applied to velocity and force feedback as given in (32) and (33) and shown in Figure 8.

$$
\begin{aligned}
& \alpha_{M}(n)= \begin{cases}0 & \text { if } \quad E_{M}^{o b s}(n)>0 \\
-\frac{E_{M}^{O b s}(n)}{V_{M}^{2}(n)}\end{cases} \\
& \alpha_{S}(n)= \begin{cases}0 & \text { if } \quad E_{S}^{\text {obs }}(n)>0 \\
-\frac{E_{S}^{O b s}(n)}{f_{S}^{2}(n)}\end{cases} \\
& f_{M}(n)=f_{M}^{\prime}(n)+\alpha_{M}(n) V_{M}(n) \\
& V_{S}(n)=V_{S}^{\prime}(n)+\alpha_{S}(n) f_{S}(n)
\end{aligned}
$$

\section{EXPERIMENTAL RESULTS}

The performance of passivity control with TDPN for telecontrol of mobile robot AutoMerlin has been plotted and presented in this section. The performance of the controller has been tested without and with the obstacles around the robot in the remote environment. The first test run was performed when the environment was free of obstacles. Fig. 9, shows offset between two velocities due to the time delay. The dots represent the random time delay between two sample times. It is vivid from Fig. 9, that there is no surge in slave velocity after passing through a communication channel with random delay. Blue plot is energy input and green plot energy output on master and slave side respectively in Fig. 10. The output energy has surge in it due to active energy. Hence, its value is greater than the input energy as depicted in the Fig. 10. The slave controller measures the active energy and diffuses its effect by dissipating it so that the forward communication always remains passive as drawn in Fig. 11, by the blue line. Force feedback is sent back from the remote environment to master haptic device. Fig. 12, is showing the force on the remote robot as a blue line and force feedback on the haptic device as a green 
line with the random delay indicated by dots. Fig. 13, shows the input energy entering the port from the slave side with the blue line and green line plot is the output energy on the other side. The surplus energy is being added by the network and due to this addition, the system output energy is greater than respective input energy. As the force on both sides is similar, the master controller is dissipating the effect of active energy as shown in Fig. 14. Whenever there is an active behavior by the network on either or both side the master and/or the slave controllers dissipate the same amount of active energy so that the system remains passive and hence stable.

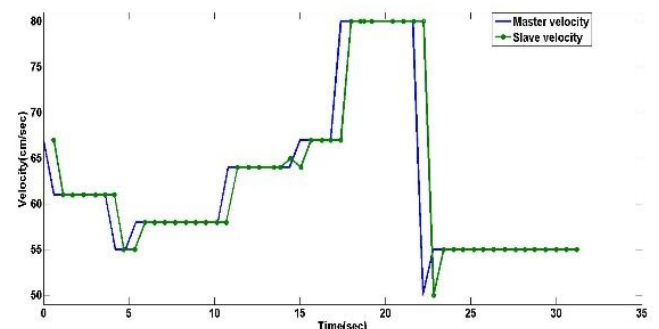

Figure 9. Linear velocities of both robots.

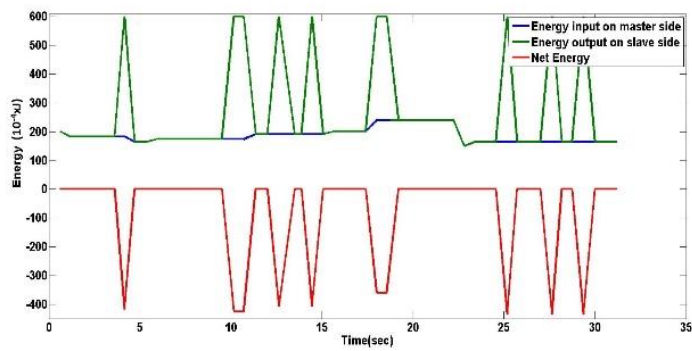

Figure 10. Energy flow master to slave.

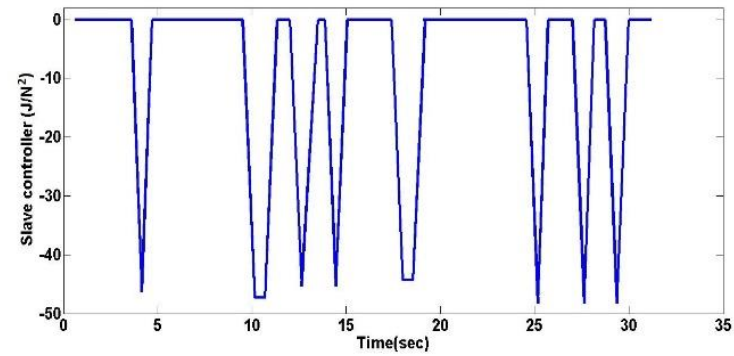

Figure 11. ${ }^{\alpha}$ controller

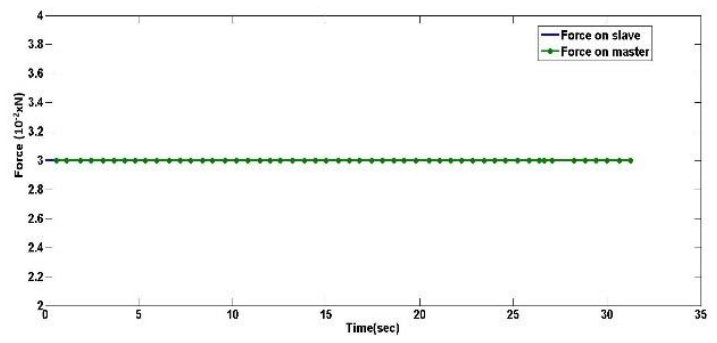

Figure 12. Force comparison on both robots. 


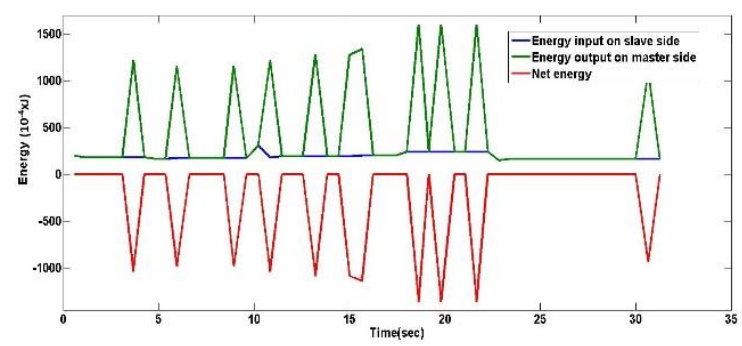

Figure 13. Energy flow slave to master.

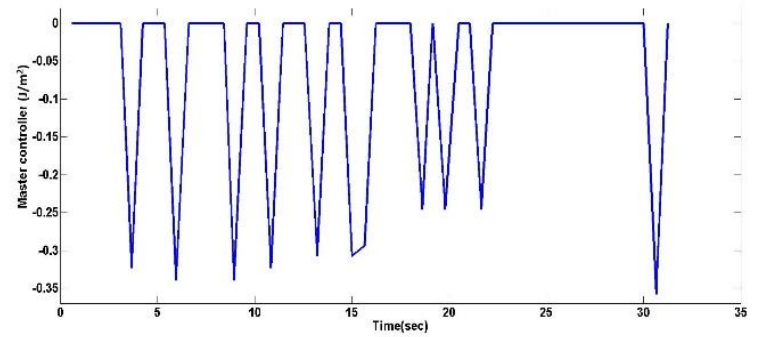

Figure 14. $\alpha_{M}$ controller.

The second test run was done when there were obstacles in the environment to evaluate the performance of the controller. The following plots show the performance of the controller with obstacles around the operational area of the remote slave robot. The Fig. 15, shows that there is no change in velocities and Fig .18, shows the force on both the slave robot and the master robot is the same. The dots indicate the random intervals. The rise in slave force is due to the unstructured environment with obstacles. This rise in force is vividly seen in Fig. 18. Similarly, the plots show that whenever there is an active behavior both $\alpha_{M}$ and $\alpha_{S}$ dissipate energy so that a stable and passive telecontrol can be performed

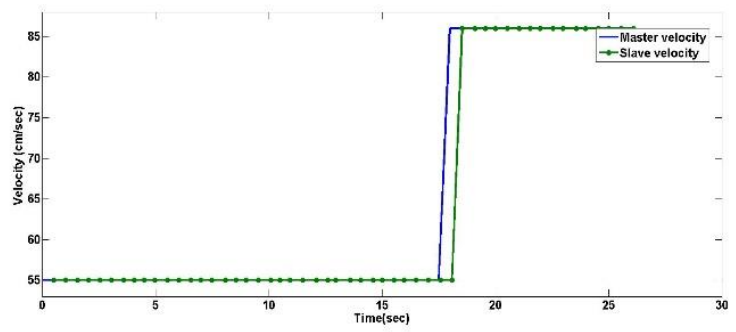

Figure 15. Linear velocities of both robots.

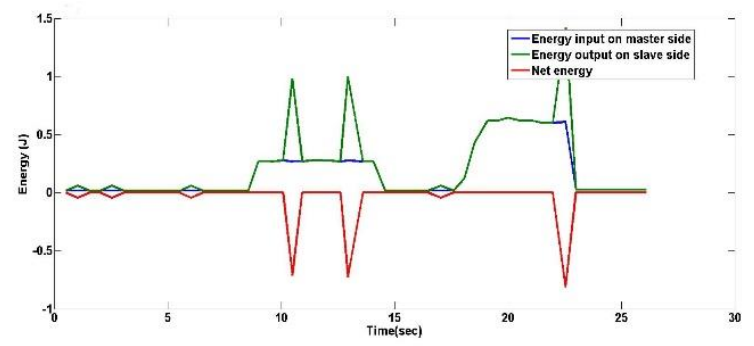

Figure 16. Energy flow master to slave. 


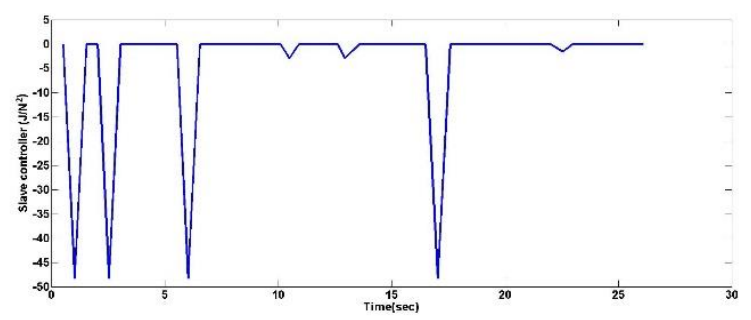

Figure 17. ${ }^{\alpha}$ controller

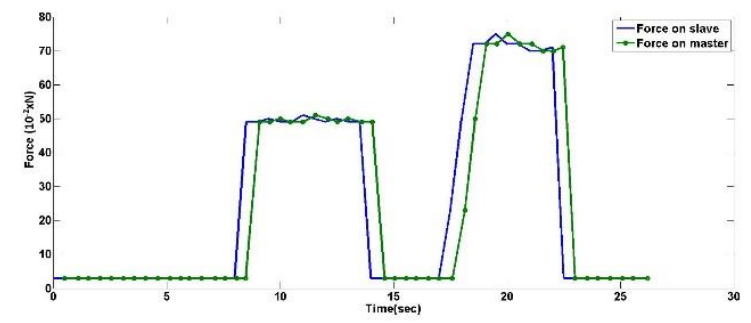

Figure 18. Force comparison on both robots.

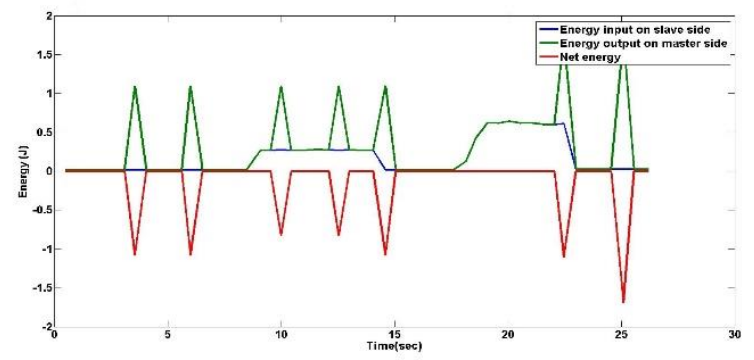

Figure 19. Energy flow slave to master.

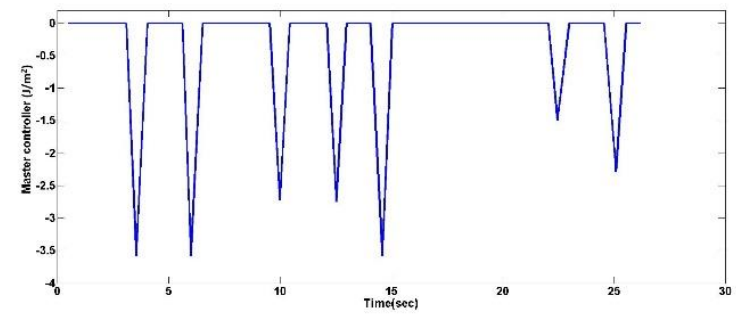

Figure 20. $\alpha_{M}$ controller.

\section{CONCLUSION AND FUTURE WORK}

The performance of the designed controller is excellent and it is keeping the system stable and passive all times. There is no compromise on unknown parameters because the controller is not based on the mathematical model of the system. It only functions when required. In order to extend this work, there are different tasks under planning like the addition of more robots for the telecontrol of a team of robots for multi-tasking.

\section{REFERENCES}

[1] A. Shahzad and H. Roth. "Teleoperation of mobile robot using event based controller and real time force feedback," Scientific Cooperations, International Workshops on Electrical and Computer Engineering Subfields, Koc University, Istanbul/Turkey, 22-23 August 2014. 
[2] A. Shahzad, R. Al-jarrah and H. Roth, "Telecontrol of AutoMerlin robot by employing fuzzy logic", International Journal of Mechanical Engineering and Robotics Research (IJMERR), Volume 5, No. 1, January, 2016.

[3] A. Shahzad, R. Al-jarrah and H. Roth, "Teleoperation of AutoMerlin by inculcating FIN algorithm", International Journal of Mechanical Engineering and Robotics Research (IJMERR), Volume 5, No. 1, January, 2016.

[4] A. Shahzad, H. Roth, "Bilateral telecontrol of AutoMerlin mobile robot", 9Th IEEE international conference on open source systems and technologies, Lahore, Pakistan, 17-19 December, 2015.

[5] V. Chawda and M. K. O’Malley," Position synchronization in bilateral teleoperation under time-varying communication delays," Ieee/asme transactions on Mechatronics, Volume 20, Issue 1, Feburary, 2015.

[6] R. Anderson and M. Spong, "Bilateral control of teleoperators with time delay," IEEE Trans. Autom. Control, vol. 34, no. 5, pp. 494-501, May.1989.

[7] R. J. Anderson and M. W. Spong, "Asymptotic stability for force reflecting teleoperators with time delay,” Int. J. Robot. Res., vol. 11, no.2, pp.135-149, 1992.

[8] H. K. Khalil, Nonlinear Systems, vol. 3. Upper Saddle River, NJ, USA,Prenticehall, 2002.

[9] K. Hashtrudi-Zaad and S. E. Salcudean, "Analysis of control architectures for teleoperation systems with impedance/admittance master and slave manipulators," Int. J. Robot. Res., vol. 20, no. 6, pp. 419-445, 2001.

[10] A. Haddadi, "Stability, performance, and implementation issues in bilateral teleoperation control and haptic simulation systems," Ph.D. dissertation, Dept. Electr. Comput. Eng., Queen's Univ., Kingston, Canada, 2012.

[11] G. Niemeyer and J.-J. Slotine, "Stable adaptive teleoperation," IEEE J. Ocean. Eng., vol. 16, no. 1, pp. 152-162, Jan. 1991.

[12] R. Anderson and M. Spong, "Bilateral control of teleoperators with time delay,” IEEE Trans. Autom. Control, vol. 34, no. 5, pp. 494-501, May.1989.

[13] P. Arcara and C. Melchiorri, "Control schemes for teleoperation with time delay: A comparative study," Robot. Auton. Syst., vol. 38, no. 1, pp.49-64, 2002.

[14] P. F. Hokayem and M. Spong, "Bilateral teleoperation: An historical survey," Automatica, vol. 42, no. 12, pp. 2035-2057, 2006.

[15] E. Nuno, L. Basa nez, and R. Ortega, "Passivity-based control for bilateral teleoperation: A tutorial," Automatica, vol. 47, no. 3, pp. 485-495, 2011.

[16] J. Ryu, D. Kwon, and B. Hannaford, "Stable teleoperation with time domain passivity control," IEEE Trans. Robot. Autom., vol. 20, no.2, pp.365-373, Apr. 2004.J. Ryu, J. Artigas, and C. Preusche, "A passive bilateral control scheme for a teleoperator with time-varying communication delay," Mechatronics, vol. 20, no. 7, pp. 812-823, 2010.

[17] Y. Ye, Y.-J. Pan and T. Hilliard, "Bilateral teleoperation with time-varying delay: A communication channel passification approach," IEEE/ASME Trans. Mechatronics, vol. 18, no. 4, pp. 1431-1434, Aug. 2013. 
Automerlin Mobile robot's bilateral telecontrol with random delay

DOI: http://dx.doi.org/10.17993/3ctecno.2019.specialissue.16 



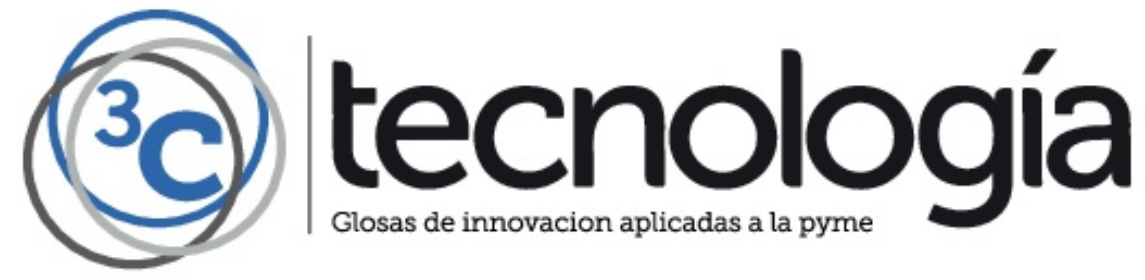

